AFOSR.TR- $78-0856$

AD A 053640

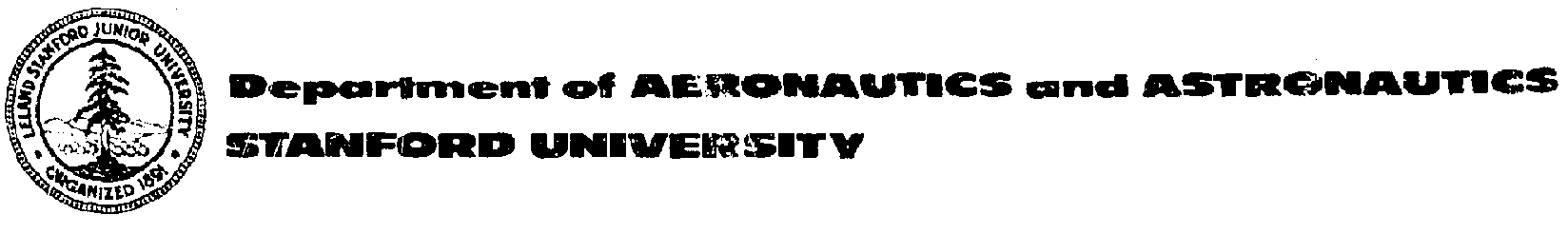

SUDAAR NO. 508

\title{
EFFECT OF CHORDWISE FORCES AND DEFORMATIONS \\ AND DEFORMATIONS DUE TO \\ STEADY LIFT ON WING FLUTTER
}

by

William Newsome Boyd

December 1977

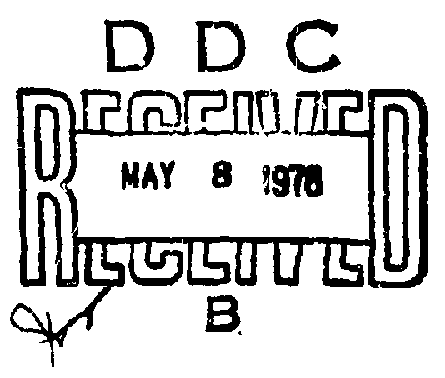

This research was supported by the Air Force Office of

Scientific Research under Contract No. AFOSR-74-2712.

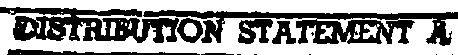

Apreverd be pabliks solecises

Orbabater Derbeitud 
ATR YORCE OFFTCE OF SCIENTIYIC RESEARCE (ATSC) WORICE OP TRLY SMITIAL TO DDC

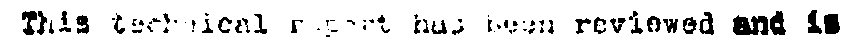

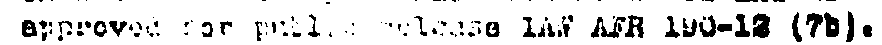
Distribition is wi:libited.

A. D. BLtise

Tachnteal Inforation offeor 


\title{
Department of Aeronautics and Astronautics Stanford University \\ Stanford, california
}

\begin{abstract}
EFFECT OF CHCIRDWISE FORCES AND DEFORMATIONS AND
DEFORMATION DUE TO STEADY IIFT ON WING FLUTTER
\end{abstract}

by

William Newsome Boyd

SUDAAR NO. 508

December $\quad .977$

This research was supported by the Air Force Office of Scientific Research under Contract No. AFOSR-74-2712. 
This investigation explores the effects of chordwise forces and deformations and steady-state deformation due to lift on the static and dynamic aeroelastic stabllity of a uniform cantilever wing. Results of this analysis are believed to have iractical applications for highperformance sallplanes ard certain RPV's.

The airfoil cross section t.s assumed to be symmetric and camber bending is reglected. Motions in vertical bending, fore-and-aft bending, anc torsion are considered. A differentlal equation model is fieveloped, which includes the nonlinear elastic bending-torsion coupling that accompanies fiven moderate deflections. A linearized expansion in small time-dependent perturbation deflections is made about a steady flight condition. The stabiltty dererminant of the linearized system then contains coefficients that depend on steady displacements. Loads derived from two-dimensional incompressible aerodynamic theory are used to obtain the majority of the results, but cases using three-dimensional subsontc compressible theory are also studied.

The stability analysis is carried out in terms of the dynamically uncoupled natural modes of vibration of the uniform cantilever. Dynamic stability in the case of incompressible strip-theory airloads is determined in two ways. One is the "V-g method" familtar to aeroelasticiars. When steady deformactions are present this method requires an iterative matching of flutter speeds with estimated speed. The second approach involves determination of the complex elgenvalues of the aeroelastic modes at any desired flight condition. The aerodynamic loads are 
expressed In terms of the generalized Theodorsen function: elgenvalueg of the peroelasti: sygtem are located with a gradient gearch technique. The effect of steady drag on filutter of nonlifting wings using incompressibie strip-theory is studied and shown to corralate with previously known results. Next, the influence of steady lifting; deformaclons on flutter is Investigated, and flutrer modes are found that involve fore-and-aft bending motions. The significance of unsteady leading edge suction forces, which are predicted by the two-dimensional Incompressible aerodynamic theory, is then examined. Two idealized examples based upon exiscing sallplanes are analyzed.

Steady drag loads lower the flutter speed for larger aspect ratios but increase it for aspect ratiog less than a certain value. Ilvergence speed is more sensitive to steady draz, and for very high aspect ratio wings it can fall below the bending-torsion flutter speed. Steady deformatior.s due to lift always decrease the flutter speed by an amount dependent upon the aspect ratio and the fore-and-aft bending stiftness. Leading-edge suction forces increase flutter speed.

Three-dimensional steady and unsteady airloads are introduced into the V-g flutter analysis scheme, and for a Nach number of zero the role of steady lifting deformations and unsteady leading-edge suction forces is more accurately determined. The behavior predicted using strip theory loads is again observed, and the suction forces are confirmed to contribute a signiftcant stabilizing effect. Further calcuiations using high subsonic Mach numbers reveal only mild effects due to compressiblitty (disregarding unsteady chordwise loads of viscous origin). 


\section{ACKNOWLENGMENTS}

The author wishes to express his deepest gratitude to his advisor, Professor holt Ashley, for his expert advice and guidance throughout the course of the research. Thanks are also due Professor Arthux" Bryson, Jr. and Professor Mlton D. Van Dyke for taking their valuable tlme ro provide helpful comments and suggestions. Finaliy for the trying fob of typing sincere thanks go to Dorothy Gundy .

Support for this work was provided by the National Aeronautics and Space Administration under contract NGL 05-020-243. 
TABLE OF CONTENTS

Chapter $\quad$ page

ABSTRACT . . . . . . . . . . . . . . . . . . . . i i

ACKNOWLEDGMENTS . . . . . . . . . . . . . . . . . . . v

TABLE OF CONTENTS ............................ . . . . . . . . .

LIST OF FIGURES . . . . . . . . . . . . . . . . . . . . . vi

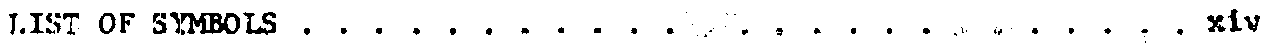

I INTRODUTITION ..................... 1

II DRAG EFFECTS OR FLUTTER AND DIVERGENCF: DEFORMATTON IN VERTICAL BENDING AND TORSION ONLY . . . . . . . 7

A. The Vertical Bending/Torsion Equations . . . . 7

B. Solution by Assuned Modos . . . . . . . . . . . 10

C. Comparison of Modal with Collocation Results . . 2.

D. Divergence Including the Effect of Drag . . . . . 24

III DEVELOPMENT OF A GENERAI SYSTEM OF EOUATIONS FOR VERTICAL BENDING/CHORDUISE BENDINE/TOKSION . . . . . 31

A. Introduction ................ 31

B. Developmerit of the Non1inent Equations of Motion . . . . . . . . . . . . . . . . . 33

C. Comparison of the VT and VCT Equations . . 45

IV FTUTTER VELOCITY CONSIDERING VERTICAL BENRING! CHORDWISE BENDING/TORSTON . . . . . . . . . . . . 53

A. Modal Equations for Smal1 Oscillatory Motions

About a Steady-State Deformation ........ 53

B. Solution Procedure for Flutter Velocity of

A Lifting Wing . . . . . . . . . . . . 64

C. The Nonlinear Elastic Coupling Terms . . . . . 65

D. Comparison of $\mathrm{V}_{i}$ and VCT Caleulations . . . 70

$V$ THE DE'IERMINATION OF AEROELASTIC MODES FOR ARBITRARY VELOCITY . . . . . . . . . . . . . . . 9I

A. Incompressik Strip-Theory Alrloads for Arofitrary Motion . . . . . . . . . . . . . . . 91

B. Solution for Acroclasta Rosta by Means of Assumed Modes . . . . . . . . . . . . . 95

C. Mode Shapes for Aeroelastic Modes . . . . . . . 100

D. Inclusion of Unsteady Chordwise Loads Due to Leading-Edge Suction............. 101 
A. Effect of Steady Brag on Flutter of a Nonlifting Wing............... 113

B. Effect of Stcady Deformations Due to Lift . . . 119

C. Effect of Unsteady Leading-Edge Suction Forices from Two-Dimensionia? Incompressible Flow . 134

i.. Two Practíca] Examples . . . . . . . . . 136

VII MUTTER VELOCITY USTNG ATRLOADS FROM THREEDIMENSTONAL SUBSONIC AERCLYNAMTC THEORY . . . . . . . 205

A. Inclusion of Three-Dimensional Afrloads . . . . 205

D. Flutter Calculation Procedite and Results . . . . 218

VIII CONCLUSIONS AND RECOMAENDATIONS . . . . . . . . . . 29

APPENDIX A: THF ASSUMED MODFS . . . . . . . . . . . . . . 243

APPENITY B: CALCLATION OF MODIFIED BESSEL FUNCTIONS : . . . . 23?

APPENDIX c: SOLUIION FOR NONLINEAR DISPLACFAENTS . . . . . . . . 23G

REFERENCES . . . . . . . . . . . . . . . . . . . . . . 243 


\section{LIST OF FICERES}

Flgure gage

2-1 Physical Origin of Drag Coupling in Eq. (2-1a) .... 29

2-2 Physical Origin of Drag Coupling in Eq. (2-16) . . 29

2-3 Comparison of Flutter Bending-Mode Shapes From Faf. 1 With Assumed Mode Results for Small- and Large-Aspect-Ratio Examples..... . . . . . . 30

3-1 Coordinate System and Displacemints......... 52

3-2 Stress Resultants and Moments . . . . . . . . . 52

4-1 Resolution of Applied Steady Loads into Components

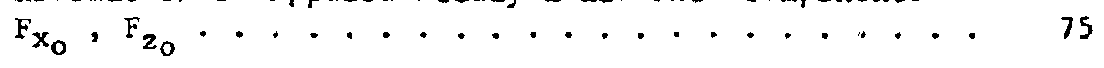

4-2 Resolution of Unsteady Airloads into Components $\mathrm{F}_{\mathrm{x}_{1}}, \mathrm{~F}_{\mathrm{z}_{1}}$. . . . . . . . . . . . . . . 75

4-3 Mass and Stiffness Matrices Appearing in Eq. (4-19) .................. 76

4-4 Flow Chart for Solution Procedure Using V-g Method .................... 78

4-5 Amplitude of the First Torsion Assumed Mode for $\alpha=.01$ and Increasing Speed, Comparing Iinear and Second-Degree Nonlinear Solutions......... 79

4-6 Amplitude of the First Vertical Bending Mode . . . . 8 8

4-7 Amplitude of the First Torsion Mode for $\alpha=0.01$ and Increasing Speed, Comparing Linear and Full Nonlinear Solutions............... 81

4-8 Amplitude of the First Vertical Bending Mode . . . . 82

4-9 Amplitude of the First Chordwise Bending Mode . . . . 83

4-10 Amplitude of the Second Torsion Mode . . . . . . 84

4-11 Amplizude of the First Vertical Bending Mode vs $\tau$, Using Second-Degree Nonlinear and Linear Approximations . . . . . . . . . . . . . . 85

4-12 Insensitivity of the Full Nonlinear Deflection of the First Vertical Bending Mode vs $\tau$, Using Full Nonlinear and Linear Approximations . . . . . . . . 
4-13 Fluter speed as a Function of Steady Wing-Tip Displaceme for Two Different Approximations in the dnalysis . . . . . . . . . . . . . .

i- li Flutete Speed as a Function of Steady Wing-Tip Mlsplacement and Chordulse Bending Stiffness . . . 88

4-15 Cosparison of Tero-lift Flutcer Speeds Found by the IT and vot Approisches .............. 89

5-1 Flow Chart for Lacating zeros of (5-8) . . . . . . 110

y-o Gradiont bearih Algorlehm ............... 111

5-3 Resalution of Unsteady Airloads Encluding Leading

Foge Suction into components $F_{n !}, F_{22} \cdot \ldots . .$. .

b-l Dependence of Fluter sneed on $c$ and $p$.

Re:. I Solut Iot................... 144

6-2 Flutter spreds. Frequencies, and Morile Shapes for

$(a, b)$ Defferent $c$ is Varles. Modal Solution of the Fis. 5-1 Confleuration ............... 145

n-3 Phasoz kepresentat ion of Elutter Mode Bending Displatents from $F i z$. h-2 for $C=0.02$ at

selerted values of $P$................

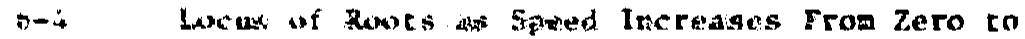

$(a-h)$ Superextesal for Ekgh Valusiz of $P$, Hith $M=40$. i. $=0.25 . \& \neq 0.1,5=0.1, n=3 \ldots . . . . .$.

6-5 Mude Shapes for Selected Rootst an Each Branch of Fif. 6-ij for $c=0 . . . . . . . . . . . .$.

6-6 Fluter Sperds. Frequencins, and Mode Shapes

(a,b) for Different $C$ as $P$ Vartes. Fir. G-2 Conflutation vith Saller Mass Rario .......

6-7 Fluter Speeds and Frequencies a: Aifected by Staady Deformations. Moderate-As pect-Ratio Example

6-8 Effect of Drap on the Relation thetween Steady Lift and Steady Bending Defiection. For the FIR- 6-7

Flucter Conditions . . . . . . . . . . . . . .

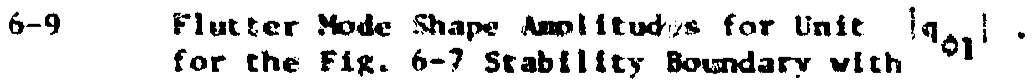

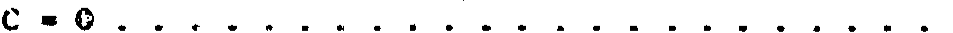


6-10 Flutter wode Shape Phase Relations for Zero Phase or $9 \phi_{1}$, for the Fig. 6-7 Stability Boundary

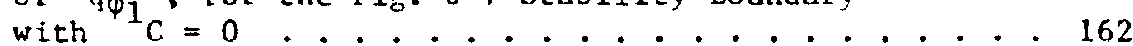

6-11 Phys :al Appearance of Flutter Mode of Deflected Wing, Fig. 6-7 Configuration with $\mathrm{C}=0 . . . . . .163$

6-12 Flutter Speed and Mode Shape Amplicudes, for Unit $\left|q_{\phi_{1}}\right|$, as Affected by Steady Deflections, Low-AspectRatio Example................... 164

6-13 Flutter Speeds as Affected by Steady Deformations, Low-Aspect-Ratio Example . . . . . . . . . . 165

6-14 FIutter Mode Shape Amplitudes for Unit $\left|q_{\phi_{1}}\right|$ Corresponding to Fig. 6-13 . . . . . . . . . . . . . 166

6-15 Flutter Mode Shape Phase Relations for Zero Phase of $q_{\phi 1}$ Corresponding to Fig. 6-13... . . . . . 167

6-16 Physical Appearance of Flutter Mode of Deflected High Aspect Ratio Wing of Fig. 6-13.......... . 168

6-17 Comparison of Drag Effect Upon Divergence and Flutter at Zero Steady Lift for the Moderate- and High-Aspect-Ratio Examples . . . . . . . . . . . 169

6-18 Effect of Siructural Damping on the Fig. 6-13

Stability Boundary ................ . 170

6-19 Locus of Roots for True Aeroelastic Modes, ModerateAspect-Ratio Example . . . . . . . . . . . . 171

6-20 Locus of Roots for True Aeroelastic Modes, HighAspect-Ratio Example . . . . . . . . . . 172

6-21 Flutcer Speeds as Affected by Steady Deformations, Large-Aspect-Ratio Example with $\tau=60 . . . . . . .173$

6-22 Flutter Mode Shape Amplitudes for Unit $\left|q_{\phi 1}\right|$ Corresponding to Fig. 6-21 ............ 174

6-23 Flutter Mode Shape Phase Relations for Zero Phase of $q_{\phi_{1}}$, Corresponding to Fig. 6-21 . . . . . . . 175

6-24 Locus of Roots for True Aeroelastic Modes, Large-

(a) Aspect-Ratio Example with $\tau=60$.......... 176

6-24 Damping Ratio of Flutter Mode Constant Speed

(b) Branches .. . . . . . . . . . . . . . 177

6-25 Physical Appearance of Flutter Modes Defined in Figs. 6-22, 23 ................. 177 
6-26 Mode Shapes for Selected Roots from Fig. 6-19. . . 178

Effect of $T$ on Flutter Speeds, ModerateAspect-Ratio Example . . . . . . . . . . . .

6-29 Effect of $\tau$ on Flutter Frequencies, Moderate-

Aspect-Ratio Example . . . . . . . . . . . .

6-30 Fffect of $T$ on Flutter Speeds, Large-Aspect-

Ratio Example....................

6-31 Effect of $T$ on Flutter Frequencies, LargeAspect-Ratio Example .. ........... 184

6-32 Effect of Ylass Racio on Divergence and Flutter, Moderate-Aspect-Ratio Example............

6-33 Effect of Mass Ratio on Divergence and Flutter, Large-Aspect-Ratio Example .............

6-34 Divergence Speeds Eor Selected Values of Drag Parameter $C$ as Aspect Ratio Parameter $P$ Varies.

6-35 Effect of Including Unsteady Leading Edge Suction Forces Upon the Constant Speed Branclies of Fig. 6-19. . . . . . . . . . . . . . . .

6-36 Drmpirg Ratio of the Branches in Fig. 6-34 . . . .

6-37 Eifect of Allowing for Unsteady Leading Edge Suction Upon the Constant Speed Branches of Fig. 20 . . . .

6-38 Damping Ratio of the Branches in Fig. 6-37, with the High Frequency Constant-Speed Branches in Fig. 6-20

Added .....................

6-39 Damping Ratio of Constant-Speed Branches in Fig. 15-24, with the Effect of Allowing for Unsteady Leading Edge Suction Also Shown ....., . . . . . . . . . .

6-40 Typical Wing Section of Gemini Sailplane Used to Determine the Six Dimensionless Parameters . . . .

6-41 Comparison of Vertical Bending Deflections of the Two Sailplane Wing Models for Given Steady Load Factor .....................

6-42 Effect of Steady Drag on Divergence and Zero sift Flutter Speeds of the Dart 27R Mode1 . . . . . . 
6-43 Effect of Steady Drag on Divergence and Zero LIft Flutter Speeds of the Gemint Model. . . . . . . 196

6-44 Stability Büutiaries for the Dart 17R Model . . . . 197

6-45 Dart 17R Fiuter Mode Shape Amplitudes,

(a) Normalized to $\left|q_{\phi_{1}}\right|$. . . . . . . . . . . . . . 198

6-45 Dart 17R Flutter Mode Shape Phase Ang?es for Zero

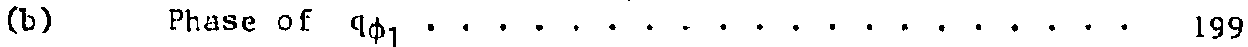

6-46 Locus of Roots for True Aeroelastic Modes, Dart l.7R Model . . . . . . . . . . . . . . . . 200

5-47 Stability Boundary Ent the Femini Model . . . . . . 201

6-48 Flutter Mode Shape Amplitudes and Phase Angles

for the Gemini Model, for Unit Amplitude and Zero

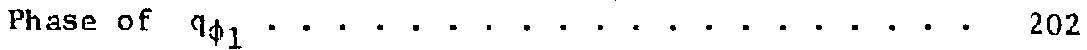

6-49 Locus of Roors for True Aeroelastic Modes, Gemint Model . . . . . . . . . . . . . . . . . 203

7-1 Flutter Speeds as Influenced by Steady Deformation, Moderate-Aspect-Ratio Example of Fig: 6-7 with $A=10$ and $M_{a}=0 . . . . . . . . . . . .225$

7-2 Fluteer Sneeds as InEluenced by Steady Deformation at Three Mach Numbers; Wing of Fig. 7-1... . . . . 226

7-3 Flutter Speeds as Influenced by Steady Deformation. Large-Aspect-Ratio Example of $\mathrm{FIg}$. 6-13 with $R=20$ and $M_{a}=0$.............. 227

A-1 Assumed Bending-Mode Shapes . . . . . . . . . 236

C-1 Elements of the Jacobian Matrix . . . . . . . . 241 


\section{LIST OF TABLES}

Table

2-1 Comparison of Assumed Mode and Collocation Methods for Predicting Flutter Speeds and

Frequencles for Three Configuratinns . . . . . . 26

2-2 Effect of Drag on Agreement of Moda1 Analysis

with Ref. I.................. . 27

2-3 Flutter Mode Shapes for Low- and High-Aspect-Ratio

Examples ............ . . . . . . . 27

2-4 Comparison of Ref. I Results with (2-29) Solutions

for Divergence Speids in the Presence of Drag . . . . 28

4-1 Comparison of Modal Flutter Speeds Computed with

the VT and VCT Systems . . . . . . . . . . . 73

4-2 Modal Convergence for Flutter at Zero Steady Lift . . 73

4-3 Moda1 Convergence for Flutter at Steady Lift for

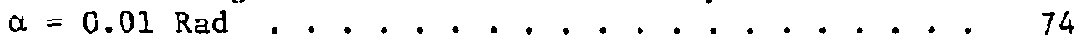

5-1 Performance of the Determinant Itteration A]gorithm for Two Cases, One Nenlifting with $n=3$ and One at Steady Lift with $\alpha=0.02$ and $n=3 \quad . .107$

5-2 Modal Convergence for Convergent and Divergent Oscillatory Aeroelastic Modes.......... 108

6-1 Modeling of the Dart 17R Wing . . . . . . . . . 142

6-2 Modeling of the Gemini Wing . . . . . . . . . . 143 


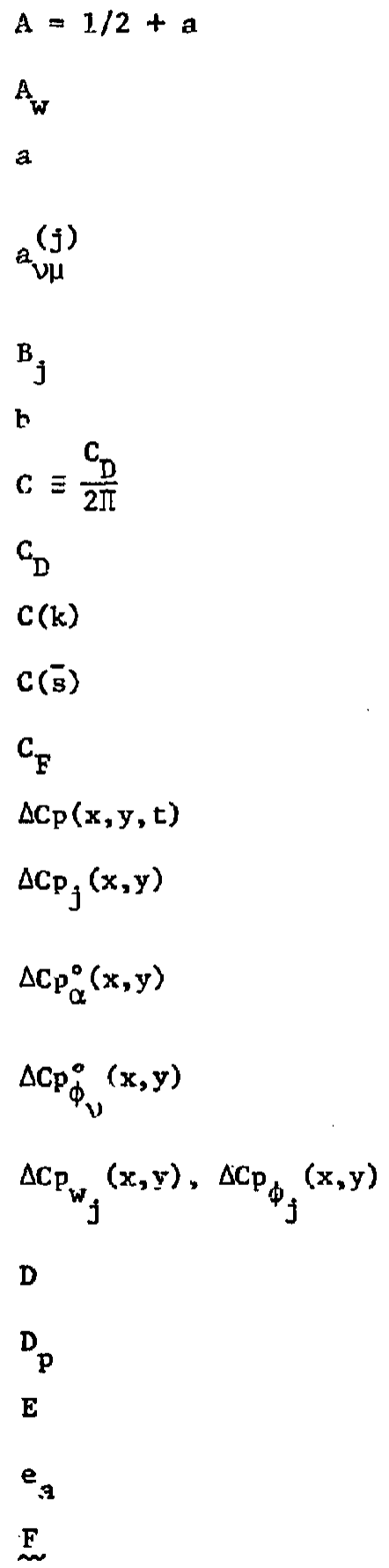

elastic axfs location parameter

structural cross-sectional area of wing

distance in semichords elastic axis is aft of midchord

pressure series coefficient for $j$ th mode, equation (7-1)

modal property defined hy equation (A-4)

semichord

drag parameter

drag coefficient

Theodorsen furcticn

generalized Theodorsen function

leading edge pressure singularity strength

pressure coefficient distribution on planform

pressure coefficient distribution for $j$ th

mode per $q_{j}$

steady pressure coefficient distribution for rigid pitch per radian

steady pressure coefficient distribution for. vth torsion mode per $q_{\phi v}^{\circ}$

oscillating pressure coefficient distributions for $j$ th bending and torsion modes

drag force

chordwise component of potential airloads

modulus of elasticity

area centroid-elastic axis offset

matrix notation for nonlinear steady equations in $(\mathrm{C}-2)$

$$
\text { r.iv }
$$




$$
\begin{aligned}
& F_{\mathbf{s}}(y, t) \\
& F_{x}, F_{z} \\
& F_{j}^{0} \\
& F_{j} \\
& F_{\alpha}^{0} \\
& F_{V}^{0} \\
& F_{w_{j}}, F_{\phi_{j}} \\
& f^{(v)}(y) \\
& E_{\phi_{i}}, E_{w_{i}}, E_{v_{i}} \\
& \text { G } \\
& \text { : } \\
& g^{(\mu)}(x) \\
& u_{v j \mu} \\
& h \\
& I_{d} \\
& I_{x} \\
& I_{z} \\
& I_{i j} \\
& I_{i f}^{(1)}, I_{11}^{(2)}, I_{i j}^{(3)}
\end{aligned}
$$

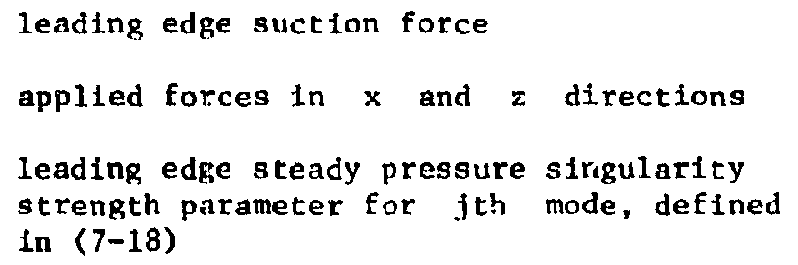




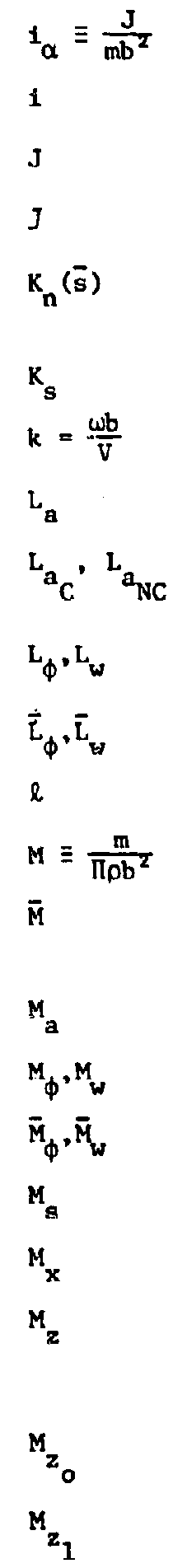

radius of gyration parameter

$\sqrt{-1}$

mass moment of inertla defined in (3-14)

Jacoblan matrix (Appendix C)

modifled Bessel function of the third kind of order $n$

$3 n \times 3 n$ stiffness matrix, Fig. 4-3

reduced frequency

gerodynamic lift

circulatory and noncirculatory parts, respectively, of lift given in $(4-14)$

expression $s$ defined in $(2-4)$

expressions defined in (5-3)

semispan

mass ratio parameter

number of downwash stations on a dowriwash chord

Msch number

expressions defined in (2-4)

expressions defined in (5-3)

3n $x$ 3n mass matrix, Fig. 4-3

stress moment defined in (3-10)

moment of applied drag loads defined in $(2-2)$, also, stress moment defined in (3-9)

steady moment defined in (3-33)

unsteady moment defined in (3-39)

xvi 
m

m

my

$\mathrm{N}_{\mathrm{j}}$

N

n

$p \equiv \frac{E I^{b^{2}}}{C I_{d} l^{2}}$
$\Delta P_{j}$
$p \equiv e \sqrt{\frac{J}{G I_{d}}}$

$Q_{\mathfrak{j}, \mathbf{l}}$

$\bar{Q}_{1,1}$

$2^{0}, 1$

9.1

$2^{\mathrm{s}}, 1$

$\mathbf{q}_{\mathrm{j}}$

$q_{\phi_{j}} \cdot q_{w_{j}} \cdot q_{f_{j}}$

$\bar{q}_{\phi_{j}}, \vec{q}_{w_{j}}, \bar{q}_{v_{j}}$

$q_{b_{j}}^{0}, q_{w_{j}}^{0}, q_{v_{j}}^{0}$

$q^{\circ}$ wing mass per unit span

gerodynamic moment

applied moment about $y$-axis

modal quantitiy defined in Appendfx A

number of downwash clords on the semispan

number of assumed modes in each degree of freedom

aspect ratio parameter

pressure distrlbut lon for fth mode per $q_{j}$

dimensionless Laplace transform variable

$3 n \times 3 n$ generalized force marrix for simple harmonlc airloads $(2 n \times 2 n$ in Chapter II)

$3 n \times 3 n$ genetalized force matrix, Laplace domain

$3 n \times .3 n+1$ generalized force matrix for steady subsonic 3-D airloads; zeroth column for rigid body pitching mode

In $x$ 3n generalized force matrix for simple harmontc subsonic 3-D alrloads, defined in $(7-30)$

sectional generaltzed force defined in (7-79)

generalized displacement (steady or simple harmontc time dependencr)

simple harmonic generalized displacements

Laplace domain generalized displacements

steady generalized displacements

vector of generalized displacement, defined in $(\mathrm{C}-1)$ 


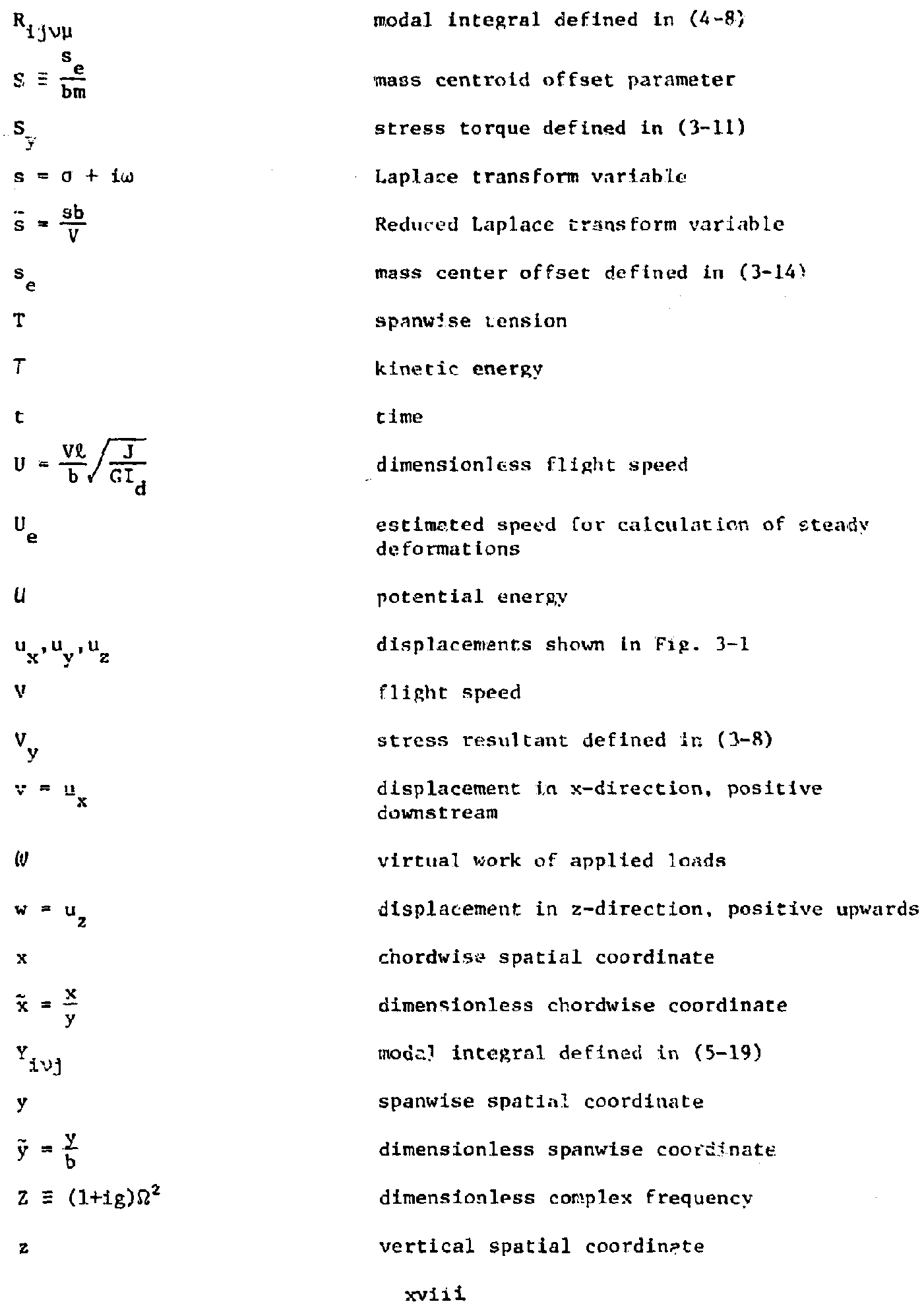




$$
z_{s}(x, y, t)
$$

$a$

8

$Y$

$\delta$

SII

$$
\varepsilon_{y y} \cdot \varepsilon_{y} \varepsilon^{* E_{y}}
$$

$\zeta$

n

$\xi$

o

$\rho_{\mathbf{s}}$

o

$\sigma_{y y}, \sigma_{y \xi}, \sigma_{y n}$

$\tau=\frac{E I_{z}}{E I_{x}}$

$\phi$

$\Omega \equiv \omega \ell \sqrt{\frac{J}{\mathbf{G I}_{\mathrm{d}}}}$

$\omega$

$\omega_{w}, w_{j}$

A diaplacement of wing planform normal to $x-y$ plane:

angle of atiack of wing root, frositive nose up quantity defined in (3-38)

discributed vorticity on alrfoll chord

titcer phase angle by which bending displacement leads corsion at wingtip

delica function

englneezing strains

damping ratio

atrfoti major principal axis roordinate

atrfoll minor princtpal axis coordinate

atmospheric density

density of ing structure

real part of $s$

stranges

bendirig stiffness ratio

torsienal displacement, positive leuding edge up

dimensionless frequency

frequency

modal natural frequenctes (Appendix A)

aspect ratio 


\section{SUPERSCRIPTS}

spanwise spatial differentlation

time differentiation

Laplace transformation

simple harmonic time dependence

\section{SUBSCRIPTS}

D

divergence

F

flutter

steady

1

unsteady

\section{ABBREVIATIONS}

Cj

Tj

vj

VT

VCT $f^{\prime}$ th chordwise bending normal mode of free vibration

$f$ 'th torsional normal mode of free vibration I'th vertical bending normal mose of free vibration

vertical bending/torsion

vertical bending/chordwise bending/torston 
Chapter I

INTRODUCTION

Aerodynamic laads and deformations parallel to the chord are usually neglected during studies of aeroelastic stablliry of lifting surfaces. Furthermore, dynamic stabllity is usually analyzed istiout regard for the influence of steady deformations due to steady state life.

Very little literature exists which treats chordwige finces and bending in an atroelastili analysis. A substantial study alt the cefects of drax iubus on divergence of a cantllever wing is made by Petre (Ref. 2, pp. 449-487). Here it is clearly demonstraced that the inceraction of drag with bending deformations due to lif:ing loads, can significantly reduce diverkence speeds. Goetz (Ref. 17) coisidered this same drag-bending deformation divergence tachantsm, speciallzed to she case of a rigid iffing surface at the end of a beam-rod. This work involvad supersonic flow pase surface having a blunt leading edge, and the resulting sizkable drag forces causted a significant reduction of the classical divergence speed.

One exaraple of an aetoelastic stuoy in which chordwise deformations of a streight cantlleve: wins are accounced for is the work in the area of tilting proprotor aircraft by Wayne Johnson. The crufsing flight condition (Ref. 27) is modeled using a proprcicor with axial flow mounted at the tip of a cantilever wing. The additional degrees of freedom associated with the individual elastlc rotor blades and the aerodynamic and inertial effects: of the proprotor result in a much wore complex and spectialized aeroelastic analysis than is considered here. 
The effect which steady chordwise forces can have upon dynamic stabllity was explored by Petre (Ref. 3) and Petre and Ashley (Ref. 1) using two-dimensional incompressible unsteady lifting airloads. The latter work presents extensive calculation:s regarding the effect of steady drag on bending-torsion flucter of a unfform cantilever wing. It serves as the starting point for the work pursued in this thesis. The objectives of this thesis are as follows:

i. To check and interpret the predicted effect of sready drag on the flutter behavior of a nonlifting wing discovered in Ref. 1, using a modal approach instead of f collocation approach.

2. To generalize the equations of notion to include consistently fore-and-aft bending motions, adequately accounting for the elastic coupling among the three degrees of freedom.

3. To include steady-state lifting deformations in the dynamic stability analysis by considering small time-dependent percurbation deflections about a steady displacement solution.

4. To allow for unsteady leading-edge-suction forces in the chordwise direction predicted by two-dimensional incompressible unstendy potential flow theory.

5. To Iraprove the representation of both steady and unsteady airloads by use of a three-dimenstonal subsonic kernel function program, from which leading-edge suction and induced drag caza also be obtained.

Items 2, 3, and 4 are interrelated and together represent a consistent extension over prevlous research in the model ing of the physical 
syster. For example, when steady lateral berding deformitelons art? present. unsteady chordwise: loads cin induce twist Ing maklons whlch signiftcanty affect dynate stabiliey. The matn purbose inere will be to establish trends and wain fundamentat instght fnto the fnflenre of cherdwise forces and steadv deformistons. hopefullv shedding liahe on their importance in practical meropace problems.

Certain assumptions are adhered to throushout this thests. Stnce the emphasis is upon working from the equations of mot ton in differential form in order to include cortaln nonl fnear elastic soupling corms. it is convenient to restrict this scudv so stright antllever wings having mass and stiffness properties unform with span. The wing is: taken to be a one-dimensional structure in the sense that all deforantions are described as functions of the spanwisc variable $y$. Uamber bending is neglected and the simple Euler-Bernoulli beam stress-stralin assumptians are used. The platform is rectangular, and the steady and unsteady flow Eields are always assumed to be superposable: unsteady loads are computed for the undeformed geometrv and apolied to the deformed wing. Although thase assumptions would be restrictive for the purpose of modeling actual structures, they are acceptable here since only the relative influences of chordise loads and steady deformations are of interest.

In Chapter II a vertical-bending/torsion model basic to the system of Ref. 1 is developed and modal equations are derived to permit flutter calculations for zero steady hift with steady draf included. Assumed mode solutions are then compared with resuits of Ref. 1. A 1inear steady-state version of the modal equations is then examined to allow computation of divergence speeds as affected by steady dras. 
In Chapter III a model central to this thesis is developed that Includes vertical bending, chordwise bending, and torsion. Nonlinear steady and linearized unsteady differentlal equations are then deduced, and they are analytically compared with the nodel in Chapter II.

In Chapter IV the modal forms of these steady and linearized unsteady equations are set up to include lifting alrloads deduced from incompressible steady and unsteady strip-theory. A scheme based on the sci-called $V-\xi$ mothod of flutter analysis is used to determine neutral dunaaic stablitity conditions, and results are checked against thoge of Chapcer II.

In Chapter $y$ a zeneralization to the case of arbitrary motion in time is presented through Laplace cransformation of the modal equations, which requires Lncompressible unsteady two-dimenstonal airloads valid for non-pertodic motions of the wing.

Adeterminant iteration procedize is used to determine the aeraelastic elgenvalues for flight speeds above and below the flutter speed. Firally, the effect of unsteady leading-edge suction forces predicted by incompresoible strip theory is included in the linearized unsteady stability system.

In Chapter VI all results for incompressible strip-theory asrloads, are assembled and systematically presented, concluding with two examples Dased upon actual sailplanes.

In Chapter VII the flutter speed prediction scheme of Chapter IV is modified to use three-dimensional subsonic steady and unsteady liliting airloads. Results are presented to indicate the effects of Enree-dimensional aerodynamics, unsteady drag, and compressibility. 
In Chapcer VIII the principal conclusions of this thesis are presented. 


\section{Chapter II}

DRAG EFFECTS ON FLUTIER ANU D TVERGENCE;

DEFORMATION TN VERTICAL BENDING AND TORSTON ONLY

\section{A. The Vertical Bending/Torsion Equat Lons}

In the Petre and Ashley work (Ref. 1) the etfect of drag on El.utter of cantlever wings $t \mathrm{~s}$ studled assuning only vertical bending and torsion about the elastic axts. The equations are cast into integra1. form, and solutions are obtained hy collocation of the integral equations at ten stattons across the span. Here we check the results of Ref. 1 using an entirely different, modal approach. The results also serve as examples with which to compare solutions found wtin the more general system. developed in the following chapter, which includes chordwise bendling.

The differential equntions of bending and torsion, as given in Ref. 1 and adapted to the present notiffan and roordinate aystem (as In Fig. 2-1), are

$$
\begin{aligned}
& (2-1 a) \quad \frac{\partial^{2}}{\partial y^{2}}\left[\operatorname{lig} \frac{\partial^{2} w}{\partial y^{2}}\right]+m \frac{\partial^{2} w}{\partial t^{2}}-s_{e} \frac{\partial^{2} \phi}{\partial t^{2}}-L_{i}(w, \phi) \\
& +\frac{\partial^{2}}{\partial y^{2}}\left(M_{z} \phi\right)=0 \\
& (2-1 b) \quad \frac{\partial}{\partial y}\left[G \tau_{i} \frac{\partial \phi}{\partial y}\right]-j \frac{\partial^{2} \phi}{\partial t^{2}}+s_{e} \frac{\partial^{2} w}{\partial t^{2}}+m_{a}(w, \phi) \\
& -M_{z} \frac{\partial^{2} w}{\partial y^{2}}=0
\end{aligned}
$$

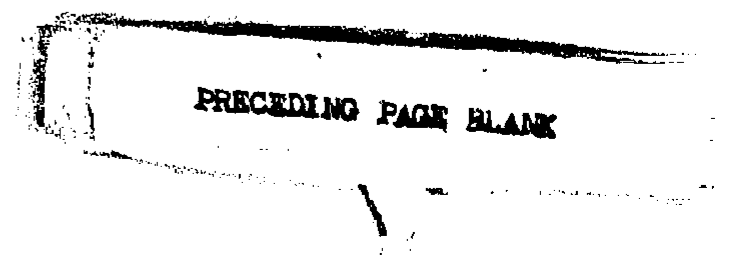


The quantizy $M_{2}$ is a function of $y$ given by

$$
\begin{aligned}
M_{z} & =-\int_{y}^{l} n(n)(n-y) d n \\
& =-D x_{2}[l-y]^{2}
\end{aligned}
$$

where it is assumed that the running drag force $D$ has constant magnitude across the span. $M_{z}$ can be recognized as the total moment about the vertical axis applied at station $y$ by all drag acting outboard of this station. Mean values of the twist and bending displacement are assumed to be zero.

Equations $(2-1)$ are linear and are coupled inertially by the third term in each; these terms arise from the offset of the center of mass of the airfoil section from the elastic axis. A second coupling effect is due entirely to drag, introduced through the terms containing $M_{z}$. All remaining terms in the equations represent the corventional elastic, Inertial, and unsteady lifting aerodynamic load contributions.

The manner In which the drag loads have been introduced into the system is discussed by Petre (pp. 449-487, Ref. 2) and can be explained by the following physically oriented argument. The drag coupiing term in the bending equation arises from the resultant bending moment $m_{x}(y)$ applied at station $y$ due to drag forces outboard of $y$. As shown in Fig. 2-1, a drag force acting outboard of station $y$ has a component $D \phi(y)$ perpendicular to the airfoil's princtpal axis of vertical bending, giving a resultant moment at $y$ about the principal direction of

$$
\begin{aligned}
m_{x}(y) & =\phi(y) D \int_{y}^{l}(n-y) d n \\
& =\phi(y) D \frac{1}{2}(l-y)^{2} \\
& =-M_{z} \phi
\end{aligned}
$$


The moment-curvature relacion for the beam,

$$
\frac{\partial^{2} w}{\partial y^{2}}=\frac{m x}{E I_{x}}
$$

together with moment-shear equilibrium

$$
\frac{\partial^{2} m x}{\partial y^{2}}=q(y)
$$

(here $q(y)$ is the positive-upward running load in the princlpal direction of the section) allows the drag effect to be expressed in equilibrium with the elastic term as

$$
\frac{\partial^{2}}{\partial y^{2}}\left[E_{x} \frac{\partial^{2} w}{\partial y^{2}}\right]+\frac{\partial^{2}}{\partial y^{2}}\left[M_{z} \phi\right]=0
$$

This is the same drag coupling term appearing in (2-1).

A similar derivation reveals the origin if the drag term in the torsion equation. Looking at the front view of the wing, (Fig. 2.2) one can see that a drag load at $n$ gives rise to a twisting moment at $y$ by acting through the moment arm given by distance $e$,

$$
e=w(\eta)-\left[w(y)+\frac{\partial w}{\partial y}(y)(n-y)\right]
$$

The resultant torque applied at station $y$ by all drag forces acting cutboard is given by

$$
T_{y}(y)=D \int_{y}^{l}\left[w(n)-w(y)-\frac{\partial w}{\partial y}(v)(n-y)\right] d n
$$

Differentiating with respect to $y$, 


$$
\begin{aligned}
\frac{d T y(y)}{d y} & =D \int_{y}^{l}\left[-\frac{\partial^{2} w(y)}{d y^{2}}(n-y)-\frac{\partial w}{\partial y}+\frac{\partial w}{\partial y}\right] d n \\
& =-D \frac{\partial^{2} w}{\partial y^{2}} \frac{1}{2}(l-y)^{2} \\
& =M_{z} \frac{\partial^{2} w}{\partial y^{2}}
\end{aligned}
$$

Elastic equilibrium for a rod loaded with applied torsion Ty(y) is given by

$$
\frac{\partial}{\partial y}\left[G r_{d} \frac{\partial \phi}{\partial y}\right]-\frac{\partial T y(y)}{\partial y}=0
$$

The drag coupling term of equation $(2-1)$ can then be identified in the result

$$
\frac{\partial}{\partial y}\left[G I \frac{\partial \phi}{\partial y}\right]-M_{2} \frac{\partial^{2} w}{\partial y^{2}}=0
$$

\section{B. Solution By Assumed Modes}

When structural dynamics problems yield solutions whose frequencies are within the range of the structure's lowest normal mode natural frequencies and the latter have a sufficiently sparse distribution, modal analysis methods prove to be effestive. Primary bending-torsion flutter of cantilever wings, under study here, is a classic example in zeroelasticity of such a system. Inclusion of steady drag effects should have only a small effect on the range of frequencles over which flutter solutions occur and, by this reasoning, should not adversely affect the convergence of modal solutions. Petre in Ref. 1 expresses 
the opinton that methods relying on the assumption of a few prescribed deformation modes constratn the flutter tnode shape and should be avolded. This opinion is tested herein by actually studying modal convergence. Solutions of (2-1) are sought using superposition of the dynamically uncoupled natural modes of free vibration in bending and torsion of a uniform cantilever beam. These assumed modes, with their relevant propertles, are described in Appendxx A. Although not true normal modes of the Inertially-coupled structure, they can be considered as "pseudoorthogonal" since integrals of the type

$$
\begin{aligned}
& \int_{0}^{i} m f_{w} f_{w j} d \tilde{y}=0 \quad 1 \neq j \\
& \int_{0}^{1} J f_{\phi_{1}}{ }_{\phi_{j}} d \tilde{y}=0 \quad i \neq j
\end{aligned}
$$

lesd to uncoupled ela'stic behavior and hence a diagonal stiffness matrix in the matrix elgenvalue problem. Use of the actual normal modes would require that they be calculated for each wing configuration studied. Since the assumed modes satisfy the naiviza? boundary conditions at the free end of the cantilever as well as the geometric boundary conditions at its clamped root, Galerkin's method can be applied to the differential equations (Ref. 4, p. 218) to obtain the system in terms of modal generalized coordinates.

To find the velocity for neutral stability (the flutter velocity), the V-g method (p. 381, Ref. 5), common in aecoelastic stability analyses, in employed. With simple harmonic motion of frequency $\omega$ the unsteady, incompressible, strip-theory lifting airloads are expressible as $[(4-123)$ and $(4-124)$ of Ref. 5] 
$(2-3 a) \quad L_{a}(w, \phi ; t)=-\Pi p b^{3} \omega^{2}\left\{-L_{w} \frac{\tilde{\omega}}{b}+\left[L_{\phi}-\left(l_{2}+a\right) L_{w}\right] \bar{\phi}\right\} e^{1 \omega t}$

$(2-3 b) \quad m_{a}(w, \phi ; t)=\pi \rho b^{4} \omega^{2}\left[-\left[M_{w}-\left(\xi_{2}+a\right) L_{w}\right] \frac{\tilde{w}}{b}\right.$

$$
\left.+\left[M_{\phi}-\left(x_{2}+a\right)\left(L_{\phi}+M_{w}\right)+\left(L_{2}+a\right)^{2} L_{w g}\right] \tilde{\phi}\right] e^{i(\Delta t}
$$

where

$(2-4) \begin{cases}\mathrm{L}_{\mathrm{w}}=1-\frac{21}{\mathrm{k}} \mathrm{C}(\mathrm{k}) & \mathrm{M}_{\mathrm{w}}=\frac{1}{2} \\ \mathrm{I}_{\phi}=\frac{\mathrm{k}}{2}-\frac{1}{\mathrm{k}}[1+2 \mathrm{C}(\mathrm{k})]-\frac{2}{\mathrm{k}^{2}} \mathrm{C}(\mathrm{k}) & M_{\phi}=\frac{3}{8}-\frac{1}{\mathrm{k}}\end{cases}$

Here $c(k)$ is the famfliar Theodorsen function of reduced frequency $k=\frac{w b}{v}$.

The drag coupling terms in $(2-1)$ can be treated as applied loads in teveloping the modal equations, by definting total applied force and moment in the bending and torsion equations as

$$
F_{z}(y, t)=L_{a}(w, \phi, t)-\frac{\partial^{2}}{\partial y^{2}}\left(M_{z} \phi\right)
$$

$$
m_{y}(y, t)=m_{a}(w, \phi, t)-M_{z} \frac{\partial^{2} w}{\partial y^{2}}
$$

Incorporation of artificial structural damping $g$ by allowing a complex elastic modulus produces the system

(2-6a) $\quad I_{x}(1+i g) \frac{\partial^{4} w}{\partial y^{4}}+m \frac{\partial^{2} w}{\partial t^{2}}-s_{e} \frac{\partial^{2} \phi}{\partial t^{2}}=F_{z}(y, t)$

$$
G I_{d}(1+1 g) \frac{\partial^{2} \phi}{\partial y^{\prime}}-J \frac{\partial^{2} \phi}{\partial t^{2}}+s_{e} \frac{\partial^{2} w}{\partial t^{2}}=-m_{y}(y, t)
$$


Formal cievelopment of the modal equations beging with substicution of the sertes expansions for $w$ and $\phi$ in terms of the assimed mode shapf: functions and generalized coordinates. With the same number $n$ of bending and torsion modes always used, the system order will be $2 n$. Generalized displacements are assumed to have simple harmonic time dependence, giving

$$
w(y, t)=\sum_{i=1}^{n} f_{w_{i}}(y) q_{w_{i}} e^{i \omega t}
$$

$(2-7 b) \quad \phi(y, t)=\sum_{1=1}^{n} F_{\phi_{1}}(y) q_{\phi_{1}} e^{i(u t}$

Generalized displacements for bending modes have units of length, where as for torsion they are dimensionless.

Galerkin's method involves substitution of (2-7) into the system represented by $(2-1),(2-2),(2-3)$, and (2-4) and then multiplication of each term in the bending equation by $f_{w_{f}}$ and each in the torsion equation by $E_{\phi_{j}}$, followed by integration across the half span. With $\mathrm{e}^{\text {iwt }}$ cancelled the resulting system is

$$
\begin{aligned}
& E I_{x}(1+i g) \int_{0}^{l} \sum_{i=1}^{n} f_{w_{1}}^{\prime} \cdot \hat{t}_{w_{1}} q_{w_{1}} d y-m w^{2} \int_{0}^{l} \sum_{i=1}^{n} f_{w_{i}} f_{w_{j}} q_{w_{1}} d y \\
& +w^{2} s_{e} \int_{0}^{l} \sum_{i=1}^{n} f_{\phi_{i}} f_{w_{1}} q_{\phi_{1}} d y=\frac{1}{e^{l w t}} \int_{0}^{l} F_{z}(\phi, w ; t) f_{w_{j}} d y
\end{aligned}
$$

$(1 \leq j \leq n)$

$$
\begin{aligned}
& \text { (2-zb) } \quad G I_{d}(I+1 g) \int_{0}^{l} \sum_{i=1}^{n} f_{\phi_{1}^{\prime}}^{\prime} \varepsilon_{\phi_{j}} q_{\phi_{1}} d y+J \omega^{2} \int_{0}^{l} \sum_{i=1}^{n} f_{\phi_{i}} f_{\phi_{j}} q_{\phi_{1}} d y \\
& -w^{2} s_{e} \int_{0}^{l} \sum_{i=1}^{n} f_{w_{i}} f_{\phi_{j}} q_{w_{i}} d y=-\frac{1}{e^{I \omega t}} \int_{0}^{l} m_{y}(\phi, w ; t) f_{\phi_{j}} d y
\end{aligned}
$$


The generalized forces can be arranged in terms of dimenstonlegs unit generalized forces $Q_{j 1}$ through the following definitions:

(2-9a) $\quad \int_{n}^{\ell} F_{z}(\phi, w ; t) E_{w j} d y=\pi \rho w^{2} b^{3} l\left[\sum_{L=1}^{n} Q_{j, 1} \frac{q_{w}}{b}+\sum_{1=1}^{n} Q_{1,1+n^{q} \phi_{j}}\right] e^{1 w t}$

(2-9b) $\quad \int_{0}^{l} m_{y}(\phi, w ; t) \dot{E}_{\phi_{j}} d y=i \rho w^{2} b l\left[\sum_{i=1}^{n} \eta_{j+n, 1} \frac{q_{w}}{b}+\sum_{i=1}^{n} q_{j+n, 1+n} q_{\phi_{1}}\right] e^{l w t}$

The modal integral in the flrst term of (2-8a), Integrated by parts twice and with application of cantllever boundary condtetons, yields

$$
\begin{aligned}
\int_{0}^{l} F_{w_{i}}^{\prime \prime \prime} f_{w_{j}} d y & =\int_{0}^{l} f_{w_{1}}^{\prime \prime} f_{w_{1}^{\prime \prime}}^{\prime} d y \\
& =0 \text { for } i \neq j
\end{aligned}
$$

From Appendix A, the $1=1$ term can be expressed in terms of the natural frequency of the $j_{\text {th }}$ assumed bending mode by the substicution

$$
\text { (2-10) } \quad E I_{x} f_{0}^{l}\left(f_{w_{j}}^{\prime \prime}\right)^{2} d y=m w_{w_{j}}^{2} \int_{0}^{l} f_{w_{j}}^{2} d y=m w_{j}^{2} l
$$

Similarly, integracing the first term in $(2-8 b)$ by parts and introducing the hatural frequency $\omega_{\phi}$ of the $j$ th assumed torsion mode leads to

$$
G I_{A} \int_{0}^{\ell} f_{\phi_{1}^{\prime}}^{\prime \prime} f_{\phi_{j}} d y= \begin{cases}0 & (1 \neq j) \\ -\frac{k}{2} J \omega^{2} \phi_{j} \ell & (1=j)\end{cases}
$$

Insertion of $(2-9),(2-10)$, and $(2-11)$ into $(2-8)$ and further use of modai orthogonality properties gaves 
$(2-12 a)$

$$
\begin{aligned}
& \frac{m}{\pi \rho b^{2}}(1+i g) \frac{w_{w}^{2}}{w^{2}} \frac{c^{i} w_{1}}{b}-\frac{m}{\Pi \rho b^{2}} \frac{q_{1}}{b}+\frac{s_{e}}{b m} \frac{m}{\pi \rho b^{2}} \sum_{1=1}^{n} I_{i j} q_{\phi_{i}} \\
& +\sum_{i=1}^{n} q_{j, i} \frac{q_{w_{1}}}{b}+\sum_{i=1}^{n} Q_{j, i+n} q_{\phi_{1}}=0
\end{aligned}
$$

$(1 \leq 1 \leq n)$

$$
\begin{aligned}
& \frac{m}{\pi \rho b^{2}} \frac{J}{m b^{2}} \frac{I_{d}}{j} \frac{w_{\phi}^{2}}{w^{2}} q_{\phi_{j}}-1_{i} \frac{m}{\pi \rho b^{2}} \frac{J}{m b^{2}} q_{\phi_{j}} \\
& \quad+\frac{s}{b m} \frac{m}{\pi \rho b^{2}} \sum_{i=1}^{n} I_{i j} \frac{q_{w_{i}}}{b}+\sum_{i=1}^{n} \eta_{j+n, i} \frac{q_{w_{i}}}{b}+\sum_{i=1}^{n} Q_{j+n, i+n} q_{\phi_{i}}=0
\end{aligned}
$$

where the bending equation has been divided through by $\Pi_{p} \omega^{2} b^{3} \ell$ and the torsion equation by $\Pi \rho \omega^{2} b^{4} \ell$. Inertial coupling, a consequence of nonorthogonality between bending and torsion assumed modes, produces terms in the modal integrals

$$
I_{i j} \equiv \int_{0}^{1} f_{w_{i}}(\tilde{y}) f_{\phi_{j}}(\tilde{y}) d \tilde{y}
$$

For now it is convenient to reference $\omega$ to the first assumed torsion mode naturisl frequency $\omega_{\phi_{1}}$. Assumed mode natural frequencies (Appendix A) are

$$
\left\{\begin{array}{l}
\omega_{\phi_{j}}^{2}=\frac{2 j^{-1}}{2} \frac{\pi^{2}}{\ell^{2}} \frac{G I_{d}}{J} \\
w_{w_{j}}^{2}=N_{j} \frac{4 \pi^{4}}{\ell^{4}} \frac{E I_{x}}{m}
\end{array}\right.
$$

Rattos that are useful are then 


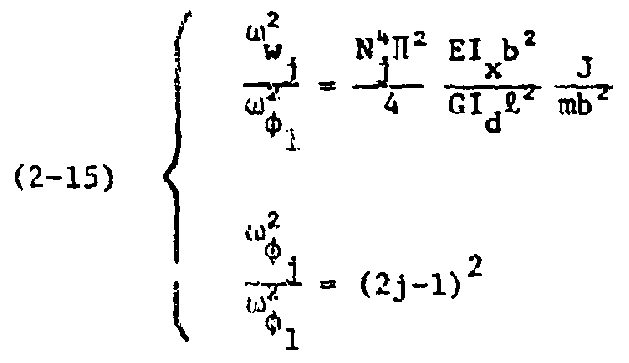

The transcendental $N_{j}$ are given in Appendix $A$ for $1 \leq 1 \leq 5$.

The equations can be nondimensionalized through the following

dimensionless parameters, chosen to be as consiatent as possible with Ref. I notation.

$(2-16) \begin{cases}M \equiv \frac{m}{\pi \rho b^{2}} & s \equiv \frac{s}{b m} \\ p \equiv \frac{E I_{x} b^{2}}{G I_{d} l^{2}} & 1_{\alpha} \equiv \frac{J}{m b^{2}}\end{cases}$

Frequency is nondimensionalized as in Ref. i by defining the frequency parameter

$$
\begin{aligned}
Z \equiv(1+i g) \Omega^{2} & \equiv(1+i g) \frac{G I}{J \ell^{2}} \frac{d}{\omega^{2}} \\
& =\frac{4}{\pi^{2}} \frac{\omega_{\phi}^{2}}{\omega^{2}}(1+i g)
\end{aligned}
$$

Substitution of $(2-15),(2-16)$, and $(2-17)$ into $(2-i 2)$ ylelds the modal equations in a suitable form for computation.

$$
\begin{aligned}
(2-18 a) \quad\left(M-N_{j}^{4} \Pi^{4} M P i_{\alpha} z\right\} \frac{w_{j}}{b} & +\sum_{i=1}^{n} Q_{j, i} \frac{q_{1}}{b} \\
& +\sum_{i=1}^{n}\left\{M S I_{j 1}+Q_{j, 1+n}\right\} q_{\phi_{1}}=0
\end{aligned}
$$


$(2-18 b)$

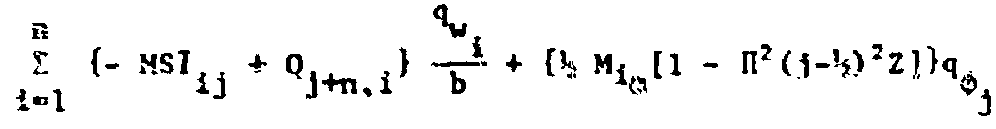

$$
\begin{aligned}
& +\sum_{i=1}^{n} Q_{j+n, i+n} Q_{\phi_{i}}=0
\end{aligned}
$$

The flutter determinane, of order $2 n$, results as a necessary condition for a nontrivial solution and leads to a complex efgenvalue probsem to determine 2 .

Next, the unit kenexalized forces $Q_{i j}$ must be expressed to allow numerical computation. Combining (2-3), (2-4), and (z-7) into (2-9)

leads to

$$
\begin{aligned}
(2-19 a)- & -\operatorname{lob}^{3} w^{2}\left\{-\int_{0}^{l} L_{w} \sum_{i=1}^{n} f_{w_{i}}(y) f_{w_{j}}(y) \frac{q_{w}}{b} d y+\int_{0}^{l}\left[L_{\phi}-\left(l_{2}+a\right) L_{w}\right] .\right. \\
& \left.\cdot \sum_{i=1}^{n} f_{\phi_{i}}(y) f_{w_{j}}(y) q_{\phi_{i}} d y\right\}+\int_{0}^{l} \frac{\partial^{2}}{\partial y^{2}}\left[\frac{\rho}{2} v^{2} b C_{D}(l-y)^{2} \sum_{i=1}^{n} f_{\phi_{i}}(y) q_{\phi_{i}}\right] .
\end{aligned}
$$

$(1 \leq f \leq n)$

$$
\text { - } f_{w_{j}}(y) d y=\pi i \rho w^{2} b^{3} \ell\left[\sum_{i=1}^{n} Q_{j, i} \frac{q_{w_{i}}}{b}+\sum_{i=1}^{n} Q_{j, i+n} q_{\phi_{i}}\right]
$$

(2-19b) $\quad \pi \rho b^{4} w^{2}\left[\int_{0}^{l}\left[-M_{w}+\left(v_{2}+a\right) L_{w}\right] \sum_{i=1}^{n} f_{w_{i}}(y) f_{\phi_{j}}(y) \frac{q_{w}}{b} d y\right.$

$$
\begin{aligned}
& \left.+\int_{0}^{l}\left[M_{\phi}-\left(l_{2}+a\right)\left(L_{\phi}+M_{w}\right)+\left(x_{2}+a\right)^{2} L_{w}\right] \sum_{i=1}^{n} f_{\phi_{1}}(y) f_{\phi_{j}}(y) q_{\phi_{i}} d y\right\} \\
& +\int_{0}^{l} \frac{1}{2} \rho v^{2} b^{2}(l-y)^{2} C_{D_{i=1}} \sum_{i=1}^{n} f_{w_{i}}^{n}(y) E_{\phi_{j}}(y)-\frac{w_{i}}{b} d y \\
& =\pi \rho w^{2} b^{4} l\left[\sum_{i=1}^{n} q_{j+n, 1} \frac{q_{w_{i}}}{b}+\sum_{i=1}^{n} n_{j+n, i+n} q_{\phi_{i}}\right]
\end{aligned}
$$

After further reduction, with

$$
v^{2}=\frac{\omega^{2} b^{2}}{k^{2}}
$$


and carrying out remaining differentiations in $y,(2-19)$ becomes:

(2-20a) $\quad \sum_{1=1}^{n}\left\{\sum_{w} \int_{0}^{1} f_{w_{1}}(\tilde{y}) f_{w_{j}}(\tilde{y}) d \tilde{y}\right\} \frac{q_{w}}{b}+\sum_{i=1}^{n}\left\{\left[-L_{\phi}+(1 / 2+a) L_{w}\right]\right.$.

$\cdot \int_{0}^{1} f_{\phi_{i}}(\tilde{y}) f_{w_{j}}(\tilde{y}) d \tilde{y}+\frac{c_{D}}{2 \pi k^{2}}\left[2 \int_{0}^{1} f_{\phi_{i}}(\tilde{y}) f_{w_{j}}(\tilde{y}) d \tilde{y}-4 \int_{0}^{1}(1-\tilde{y}) \cdot\right.$

$(1 \leq j \leq n)$

- $\left.f_{\phi_{i}}^{\prime}(\tilde{y}) f_{w_{j}}(\tilde{y}) d \tilde{y}+\int_{0}^{1}(1-\tilde{y})^{2} f_{\phi_{i}^{\prime \prime}}^{\prime}(\tilde{y}) f_{w_{j}}(\tilde{y}) d \tilde{y}\right] q_{\phi_{i}}$

$=\sum_{i=1}^{n} Q_{j, i} \frac{q_{w}}{b}+\sum_{i=1}^{n} Q_{j, i+n} q_{\phi_{i}}$

$(2-20 b) \quad \sum_{i=l}^{n}\left\{-M_{w}+\left({ }_{2}+a\right) L_{w}\right] \int_{0}^{1} f_{w_{j}}(\tilde{y}) f_{\phi_{j}}(\tilde{y}) d \tilde{y}$

$\left.+\frac{C_{D}}{2 \pi k^{2}} \int_{0}^{1}(1-\tilde{y}){ }^{2} f_{w_{i}}^{\prime \prime}(\tilde{y}) f_{\phi_{j}}(\tilde{y}) d \tilde{y}\right\} \frac{w_{i}}{b}+\sum_{i=1}^{n}\left\{\left[M_{\phi}-\left(1_{\Sigma}+a\right)\left(L_{\phi}+M_{w}\right)\right.\right.$

$\left.\left.+\left(\xi_{z}+a\right)^{2} I_{w}\right] f_{0}^{1} E_{\phi_{1}}(\tilde{y}) f_{\phi_{j}}(\tilde{y}) d \tilde{y}\right\} q_{\phi_{i}}$

$=\sum_{i=1}^{n} Q_{j+n, i} \frac{q_{w_{1}}}{b}+\sum_{i=1}^{n} Q_{j+n, i+n} Q_{\phi_{i}}$

Here, integrals in the spanwise variable $y$ have been non-

dimensionalized. Four different types of modal integrals are encountered, including the $I_{\text {if }}$ previously identified. The three new forms arise from the drag coupling terms and are

$(2-21)\left\{\begin{array}{l}I_{i j}^{(1)}=\int_{0}^{1}(1-\tilde{y})^{2} f_{w_{i}}^{\prime \prime}(\tilde{y}) f_{\phi_{j}}(\tilde{y}) d \tilde{y} \\ I_{i j}^{(2)}=\int_{0}^{1}(1-\tilde{y}) f_{\phi_{1}}^{\prime}(\tilde{y}) f_{w_{j}}(\tilde{y}) d \tilde{y} \\ I_{i j}^{(3)}=f_{0}^{1}(1-\tilde{y})^{2} f_{\phi_{i}}^{\prime \prime}(\tilde{y}) f_{w_{j}}(\tilde{y}) d \tilde{y}\end{array}\right.$ 
The modal integrals in $(2-13)$ and $(2-21)$ were numerically computed to twelve significant digits for $1 \leq i, 1 \leq 5$.

Two additional dimensionless paraneters can be introduced, again drawn from Ref. 1.
$(2-22)$
$A=\frac{1}{2}+2$
$c=\frac{C_{D}}{2 \pi}$

The drag parameter $\mathrm{C}$ is defined as the applied steady sectional dxag coefficient divided by the sectional lift curve slope (2⿰丿⺄ fó incompresible strip theory).

After $(2-20),(2-21)$, and $(2-22)$ are cambined with orthogonal modal integrals recognized, the unit generalized forces are found to be $(2-23)\left\{\begin{array}{l}Q_{j, i}= \begin{cases}I_{w} & (i=j) \\ 0 & (i \neq j)\end{cases} \\ Q_{j, i+n}=-\left[I-A L_{w}\right] I_{i j}+\frac{C}{k^{2}}\left[2 I_{i j}-4 I_{i j}^{(2)}+I_{i j}^{(3)}\right] \\ Q_{j+n, i}=-\left[M_{w}-A L_{w}\right] I_{i j}+\frac{C}{k^{2}} I_{i j}^{(1)} \\ Q_{j+n, i+n}= \begin{cases}I_{i}\left[M_{\phi}-A\left(L_{\phi}+M_{w}\right)+A^{2} L_{w}\right] & (i=j) \\ 0 & (i \neq j)\end{cases} \end{array}\right.$

The $Q_{i j}$ depend only upon $k, A$, and $C$. Actual computation of the Theodorsen function is accomplished by direct use of the ascending power series of the modified Bessel functions $K_{0}$ and $K_{1}$, as explained in Appendix B.

Equations $(2-4),(2-18)$, and $(2-23)$ together supply the flutter determinant for the VT system using assumed modes, given in matrix 
form in (2-24). Solutions can be found using from one to five assumed modes in each of bending and corsion, and the maximum order of the determinant will be 20 .

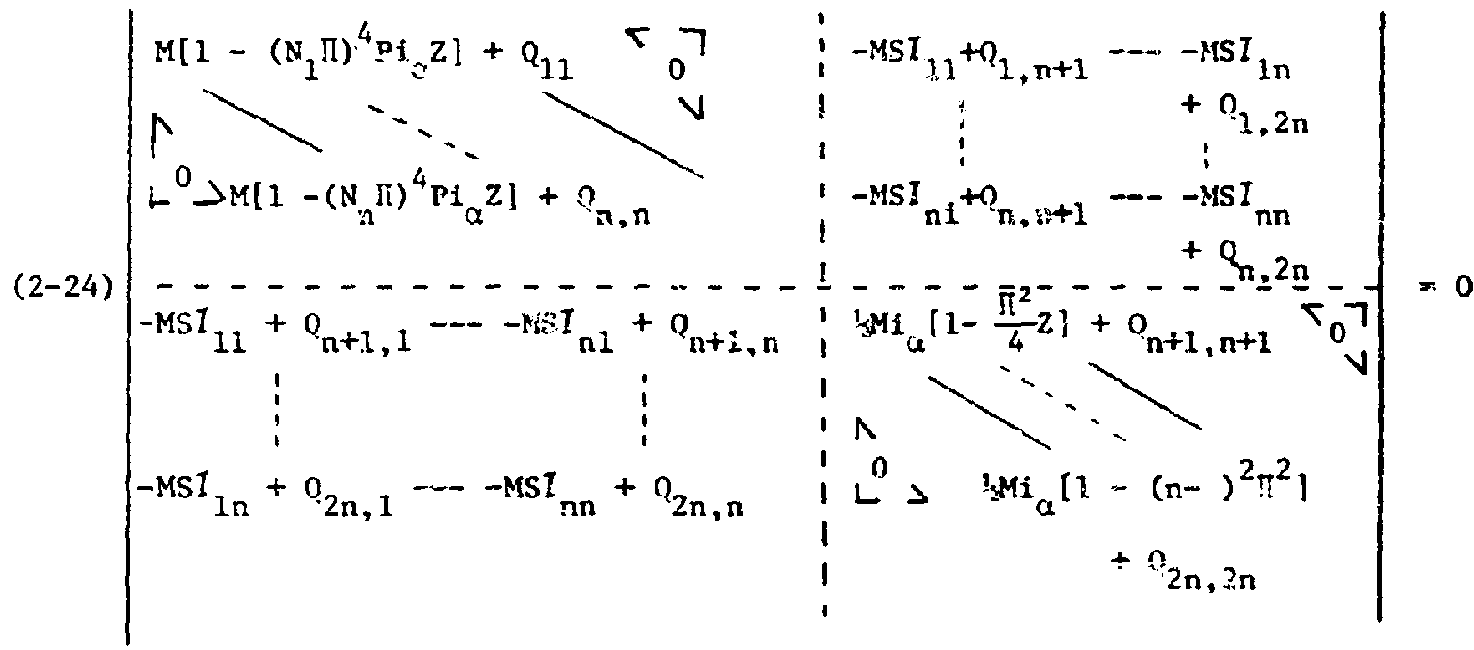

In seeking solutions, values for $M, P, I_{\alpha}, A$, and $S$ must first be chosen to specify the wing configuration. Then, for any desired value of $C$ and an estimate of reduced frequency $k$, cotsplex eigenvalues $Z$ can be found from (2-24) via linear matix eigenvalue techniques.

A computer program is used to solve for flutter conditions as Eollows. With a first estimate of $k$ chosen large enough so that the structural damping,

$(z-25) \quad g=\frac{\operatorname{Im}(z)}{\operatorname{Re}(Z)}$

is negative for all $2 n$ eigenvalues, successively smaller values of k a:re assigned and eigenvalues compuced until a positive $B$ is obtained for the eigenvalue corresponding to the aeroelastic mode which encounters flutter. Then a zero-finding subroutine locates $k$ for which $g=0$ for the flutter mode. Dimensionles: speed and frequency, defined by 


$$
U \equiv \frac{V \ell}{b} \sqrt{\frac{J}{G I}}
$$

$$
\Omega \equiv \omega l \sqrt{\frac{\mathrm{J}}{\mathrm{GI}_{\mathrm{d}}}}
$$

can easily be computed for the flutter elgenvalue by

$$
\Omega=\frac{1}{\sqrt{\operatorname{Re}(2)}}
$$

$(2-27)$

$$
\mathrm{U}=\frac{\Omega}{\mathrm{k}}
$$

The flutter mode shape is also found, since th is related to the eigenvector of the efgenvalue yielding the flutter condition.

\section{Comparison of Modal with Collocation Results}

A rather thorough study was carried out in Ref. 1, covering a wide range of practical combinations of the dimensionless parameters. The present obfective is not to recalculate all of the same data but rather to evaluate the effectiveness of the assumed-mode approach. Consequently, (2-24) has been solved at condftions parallel to ones for which resuits are publtshed in Ref. 1 to offor direct comparison. Also (2-24) is used to verify the performance of the VCT system developed in the next chapter.

A comparison of assumed mode calculations with Ref. 1 results is presented in Table 2.1 for three different conflgurations. of these, cases (a) and (c) rapresent a stubby low-aspect-ratio wing (with $P=.4$ ) whereas case (b) is the opposite extreme of large aspect ratio. A typical ratio $\frac{\mathrm{ET}_{\mathrm{X}}}{\mathrm{GI}_{\mathrm{d}}}=1.6$, for example, would fix the aspect ratio of 
cases (a) and (c) at 2 and of case (b) at 20 . The mass ratio parameter of cases (a), (b) is in the regime encountered by sailplanes whereas in (c) it is representative of conventional aircraft. Finally, the steady drag parameter is zero in the first two cases but has the extremely large value $c=0.04$ in (c).

In all cases $n=3$ already yields adequate modal convergence. For the large aspect ratio case the use of one assumed mode $(n=1)$ gives a significant error, yet $n=2$ produces good accuracy. This suggests that the second bending mode is an important factor in flutter of high-aspect-ratio wings, a phenomenon discussed in Chapter. 6 .

Additional comparisons between flutter speeds and fraquencies found by the two methods are offered in Table 2.2, emphasizing thelr relative accuracy as steady drag is increased to the very high value $C=0.04$. Evidently good agreement is maintained in the presenc. of drag.

The mode shape at flutter for two of the preceding cases is presented in Table 2.3 for wings of small and large aspect ratio, each with $\mathrm{C}=0.04$. Amplitudes of the ten generalized displacements are normalized with respect to $\left|q_{\phi_{1}}\right|$, and the phase angies are referenced to the phase of this torsion mode. For $P=0.4$ only $q_{\phi_{1}}$ and $q_{w_{1}}$ have appreciable magnitude, whereas the large-aspect-ratio example displays a significant contribution by $q_{w_{2}}$ as well.

These flutter mode shapes can be compared to the Ref. 1 results, with some effort, as follows. In Ref. 1, the tangent of the angle by which the torstonal displacement at the wingtip leads bending displacement in the flutter mode is tabulated; it $i$ 's converted here to an angle 
in degrees. The spanwise shape of the bending portion of the mode at flutter for the two Table 2.3 cases is illustrated in Fig. 5 of Ref. 1 . Since the flutter mode's bending displacement obtained in that treatment was complex, its amplitude at each spanwise station was taken, phase differences being neglected in the figure.

For the present solutions a similar assumption is made to display the bending mode shape of the $P=0.004$ case in Table 2.3 . Contributions of $q_{w_{1}}$ and $q_{w_{2}}$ have been added vectorically at points along the span to allow comparison of bending amplitudes with the Ref. 1 figure. For $p=0.4$, of course, only the first bending mode contributes significantly and phase differences are negligiblc.

The comparison of bending flutter mode shapes appears in Fig. 2-3, where amplitudes are normalized to unit torsional displacement at the tip. Phase angles $\delta$ between bending and torsion at the tip are also compared. At low aspect ratio excellent agreement for phase angles and morie shapes is observed, with mild disagreement in the bending mode antplitudes. In the $P=0.004$ case, for which the Ref. 1 solution was made using only five spanwise collocation poincs, a significant disagreement in mode shapes and tip phase angles is observed. In spite of this discrepancy, the respective flutter speeds differ by on $1 y 0.82 \%$. For all large-aspect-ratio cases compared this sort of discrepancy in flutter mode shapes is observed, where the second bending mode plays a significant role and causes appreciable phase differences in bending deflections along the span. Mode shapes at flutter are analyzed in more detail In Chapter 6. 


\section{Divergence Including the Effects of Drag}

One further application of the VT linear model is predicting the effect of drag forces, steady in direction and magnitude and uniform across the span, on static aeroelastic stability. Removal of all timedependent terms in (2-1) and tnsertion of simple strip-theory steady incompressibla aerodynamic loads gives

$$
\begin{aligned}
& E_{x} \frac{\partial^{4} w_{o}}{\partial y^{4}}-2 \Pi \rho v^{2} b \phi_{o}+\frac{\partial^{2}}{\partial y^{2}}\left(M_{z} \phi_{0}\right)=0 \\
& G I_{d} \frac{\partial^{2} \phi_{o}}{\partial y^{2}}+2 \Pi \rho v^{2} b^{2} A \phi_{o}-M_{z} \frac{\partial^{2} w_{o}}{\partial y^{2}}=0
\end{aligned}
$$

Subscripts arnphasize that deflections are static quantities.

As in the foregoing dynamic analysis, deflections are represented by assumed modes and a system of $2 \mathrm{n}$ modal equations is derived, which has a nontrivial solution only if the determinant of the matrix of coefficients is zero which yields the divergence speed with drag effects included. For brevity, since the manipulations involved are quite straightforward, the final form of the stabllity determinant is presented here.

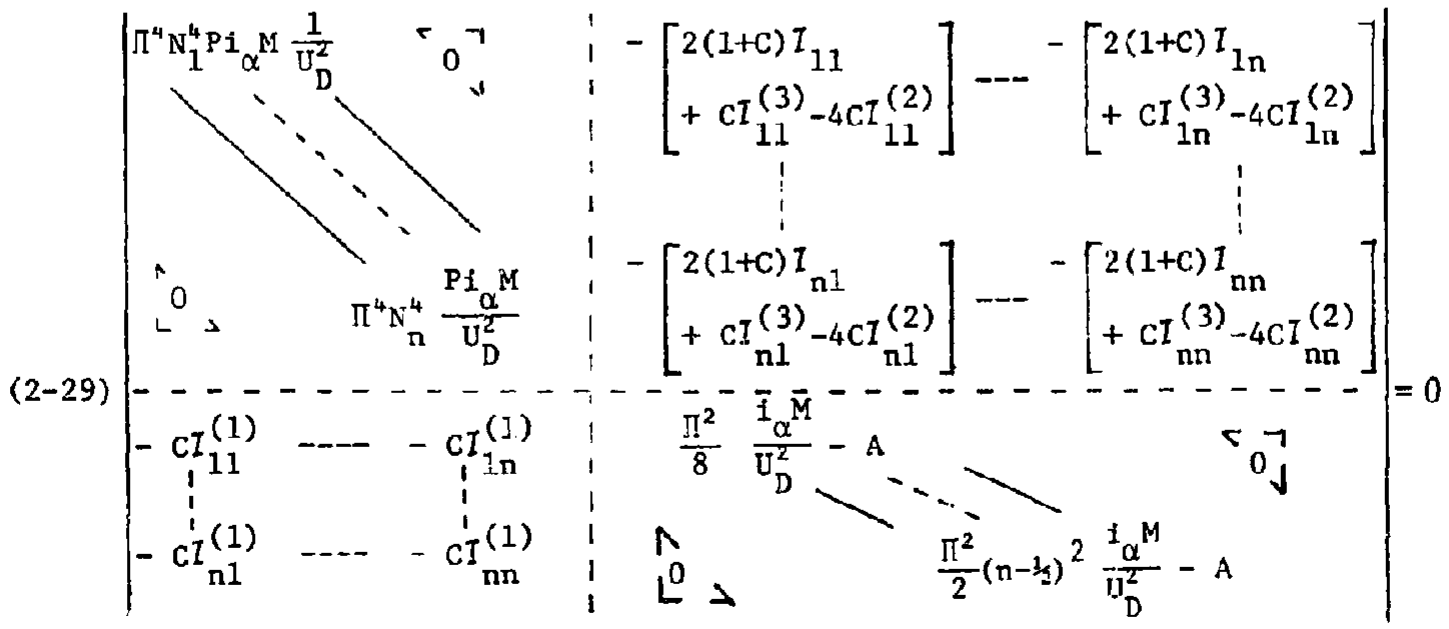


Solution of this determinant for its largest eigenvalue gives the divergence speed $U_{D}$. This is of interest in the ensuing work, particularly for studying dynamic stability of wings having steadystate lifting deflections in the presence of drag, which can reduce divergence speed considerably.

For zero steady drag $(C=0)$ the classical divergence speed comes trivialiy from (2-29), which degenerates for $c=0$. The classical divergence mode shape is fust the first assumed torsion half-sine mode, and the first torsion modol equation uncouples to give the divergence speed,

$$
U_{D}=\pi \sqrt{\frac{1}{\frac{1}{8 A}}}
$$

When $A$ is decreased to zero this classical divergence speed becomes infinite; yet when $c \neq 0 \quad(2-29)$ will still yield finite solutions with $A=0$.

A direct comparison of solutions of (2-29) with Ref. I results for the effect of drag on divergence is presented in Table 2.4. Modal convergence is satisfactory, but the modal approach appears to differ more significantly from the Ref. I analysis for divergence calculations than for flutter results. 


\begin{tabular}{|c|c|c|c|c|}
\hline Case & $n$ & $\mathrm{U}_{\mathrm{F}}$ & $\Omega_{F}$ & $\begin{array}{c}\text { \% Difference } \\
\text { of } U_{F} \text { from } n=5\end{array}$ \\
\hline $\begin{array}{c}\text { (a) } \\
M=10.0 \\
P=0.4 \\
1_{\alpha}=0.25 \\
S=0.1 \\
A=0.1 \\
C=0.0\end{array}$ & $\begin{array}{c}1 \\
2 \\
3 \\
4 \\
5 \\
(\operatorname{ReE} .1)\end{array}$ & $\begin{array}{l}2.7175179 \\
2.7239548 \\
2.7240004 \\
2.7240178 \\
2.7240199 \\
2.699\end{array}$ & $\begin{array}{l}1.3105289 \\
1.3114559 \\
1.3114641 \\
1.3114673 \\
1.3114675 \\
1.309\end{array}$ & 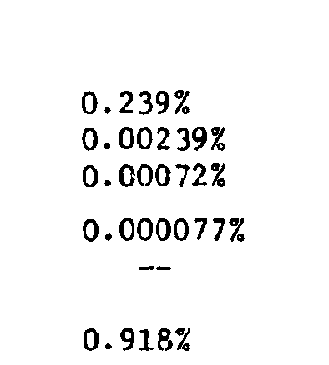 \\
\hline $\begin{aligned} & (b) \\
M= & 10.0 \\
P= & 0.004 \\
I_{\alpha}= & 0.25 \\
S= & 0.1 \\
A & =0.1 \\
c & =0.0\end{aligned}$ & $\begin{array}{r}1 \\
2 \\
3 \\
4 \\
5 \\
\text { (Ret. 1) }\end{array}$ & $\begin{array}{l}4.2621908 \\
4.0842768 \\
4.0864 .182 \\
4.0866066 \\
4.0866310 \\
4.032\end{array}$ & $\begin{array}{l}0.842707 \\
0.8849367 \\
0.8850560 \\
0.8850659 \\
0.8850650 \\
0.886\end{array}$ & \begin{tabular}{l}
$4.296 \%$ \\
$0.0576 \%$ \\
$0.0052 \%$ \\
$0.00059 \%$ \\
\multicolumn{1}{c}{-} \\
$1.337 \%$
\end{tabular} \\
\hline $\begin{aligned} &(c) \\
& M= 40 . \\
& P= 0.4 \\
& i_{\alpha}=0.25 \\
& S=0.1 \\
& A=0.1 \\
& C=0.04\end{aligned}$ & $\begin{array}{r}2 \\
3 \\
4 \\
5 \\
\text { (Ref. 1) }\end{array}$ & $\begin{array}{l}4.260873 \\
4.260879 \\
4.260882 \\
4.260889 \\
4.199\end{array}$ & $\begin{array}{l}1.294037 \\
1.2940232 \\
1.2940250 \\
1.2940236 \\
1.293\end{array}$ & $\begin{array}{c}0.00155 \% \\
0.000235 \% \\
0.00016 \% \\
--\end{array}$ \\
\hline
\end{tabular}

TABLE 2.1 Comparison of Assured Mode and Collacation Methods for Predicting Flutter Speeds and Frequencies for Tinree Configurations 


\begin{tabular}{|c|c|c|c|c|c|c|c|c|}
\hline & \multirow{2}{*}{ Case } & \multirow{2}{*}{ C } & \multicolumn{3}{|c|}{$\mathrm{U}_{\mathrm{F}}$} & \multicolumn{3}{|c|}{$\Omega_{F}$} \\
\hline & & & $\mathrm{n}=5$ & Ref. 1 & $\begin{array}{c}\% \\
\text { Difference } \\
\end{array}$ & $\mathbf{n}=\mathbf{5}$ & $\operatorname{Re} f \cdot 1$ & $\begin{array}{c}\% \\
\text { Difference }\end{array}$ \\
\hline $\begin{array}{l}\mathbf{p} \\
\mathrm{M} \\
1_{\alpha} \\
\mathrm{s} \\
\mathrm{A}\end{array}$ & $\begin{array}{l}=0.4 \\
=10.0 \\
=0.25 \\
=0.1 \\
=0.1\end{array}$ & $\begin{array}{l}0.0 \\
0.02 \\
0.04\end{array}$ & $\begin{array}{l}2.7240 \\
2.7830 \\
2.8623\end{array}$ & $\begin{array}{l}2.699 \\
2.753 \\
2.823\end{array}$ & $\begin{array}{l}0.93 \% \\
1.08 \% \\
1.37 \%\end{array}$ & $\begin{array}{l}1.3115 \\
1.3071 \\
1.3024\end{array}$ & $\begin{array}{l}1.309 \\
1.305 \\
1.300\end{array}$ & $\begin{array}{l}0.19 \% \\
0.16 \% \\
0.18 \%\end{array}$ \\
\hline $\begin{array}{l}P \\
M \\
1 \\
s \\
A\end{array}$ & $\begin{array}{l}=0.004 \\
=40.0 \\
=0.24 \\
=0.1 \\
=0.1\end{array}$ & $\begin{array}{l}0.0 \\
0.02 \\
0.04\end{array}$ & $\begin{array}{l}7.1778 \\
6.8588 \\
6.7704\end{array}$ & $\begin{array}{l}7.127 \\
6.797 \\
6.715\end{array}$ & $\begin{array}{l}0.71 \% \\
0.90 \% \\
0.82 \%\end{array}$ & $\begin{array}{l}0.8651 \\
0.9074 \\
0.9223\end{array}$ & $\begin{array}{l}0.875 \\
0.919 \\
0.934\end{array}$ & $\begin{array}{l}1.14 \% \\
1.28 \% \\
1.27 \%\end{array}$ \\
\hline
\end{tabular}

TABLE 2.2 Effect of Drag on Agreement of Modal Analysis with Ref. 1

\begin{tabular}{|c|c|c|c|c|}
\hline \multirow{2}{*}{$\begin{array}{l}\text { Generalized } \\
\text { Displacement }\end{array}$} & \multicolumn{2}{|c|}{$P=0.4$} & \multicolumn{2}{|c|}{$P=0.004$} \\
\hline & Amplitude & Phase & Amplitude & Phase \\
\hline & 0.71276 & $219.62^{\circ}$ & 1.4515 & $195.46^{\circ}$ \\
\hline & 0.00132 & $21.95^{\circ}$ & 0.6288 & $217.24^{\circ}$ \\
\hline & 0.0000271 & $150.13^{\circ}$ & 0.00449 & $203.35^{\circ}$ \\
\hline & 0.00000408 & $147.28^{\circ}$ & 0.000674 & $-10.94^{\circ}$ \\
\hline & 0.00000213 & $163.47^{\circ}$ & 0.000189 & $15 \% .41^{\circ}$ \\
\hline 9 & 1.0 & $0^{c}$ & 1.0 & $0^{\circ}$ \\
\hline 9 & 0.01503 & $222.02^{\circ}$ & 0.01454 & $147.11^{\circ}$ \\
\hline & 0.000808 & $215.78^{\circ}$ & 0.01786 & $210.39^{\circ}$ \\
\hline$q_{\phi_{u}}$ & 0.000773 & $221.31^{\circ}$ & 0.00930 & $210.13^{\circ}$ \\
\hline$q_{\phi_{s}}$ & 0.000186 & $217.90^{\circ}$ & 0.00462 & $211.73^{\circ}$ \\
\hline
\end{tabular}

TARLE 2.3 Flutter Mode Shapes for Low- and High-Aspect-Ratio Examples. $\left(M=40, i_{\alpha}=0.25, A=0.1, S=0.1, C=0.04\right)$ 


\begin{tabular}{|c|c|c|c|c|}
\hline \multirow[b]{2}{*}{$n$} & \multicolumn{2}{|c|}{$c=0.02$} & \multicolumn{2}{|c|}{$C=0.04$} \\
\hline & $u_{D}$ & $\begin{array}{l}\% \text { Difference } \\
\text { From } n=5\end{array}$ & $\mathbf{u}_{\mathrm{D}}$ & $\begin{array}{l}\% \text { Difference } \\
\text { From } n=5\end{array}$ \\
\hline 1 & 4.58288 & $2.044 \%$ & $3.90 ? 05$ & $1.999 \%$ \\
\hline 2 & 4.48660 & $-0.0999 \%$ & 3.81863 & $-0.156 \%$ \\
\hline 3 & 4.49174 & $0.0144 \%$ & 3.82 .465 & $0.0017 \%$ \\
\hline 4 & $4.4906 \pi$ & $-0.0094 \%$ & 3.82403 & $-0.0144 \%$ \\
\hline 5 & 4.49109 & -- & 2.82458 & - \\
\hline$(\operatorname{Ref}, 1)$ & 4.66 & $3.63 \%$ & 3.96 & $3.42 \%$ \\
\hline
\end{tabular}

TABLE 2.4 Comparison of Ref. 1 Results with (2-2y) Solutions for Divergence Speeds in the Presence of Drag. ( $P=0.004$, $M=40 ., i_{\alpha}=0.25, A=0.1$ ). (For $C=0, U_{D}=11.1072$ ) 

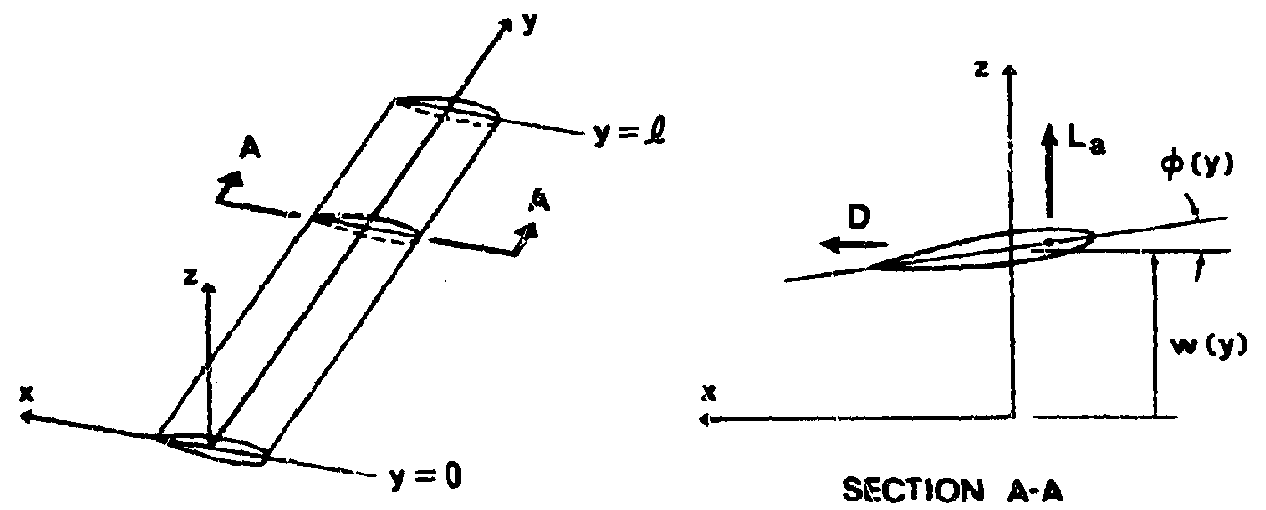

FIGURE 2-1 Physical Origin of Drag Coupling in Eq. (2-1a)

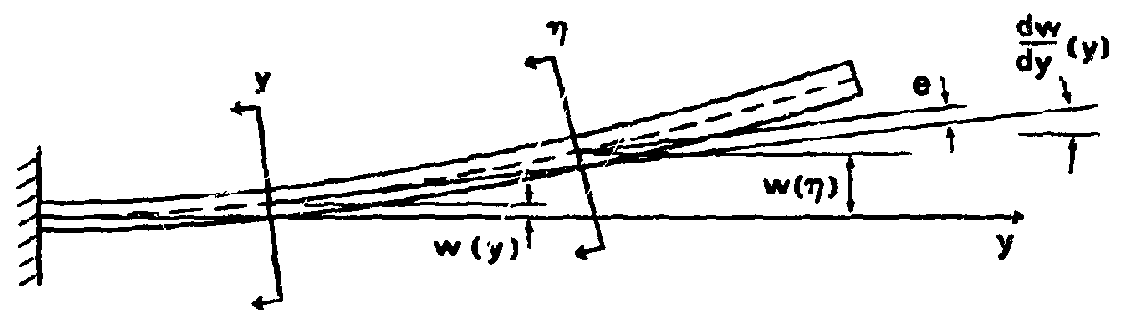

FrounE 2-2 Physical Origin of Drag Coupling in Eq. (2-1b) 


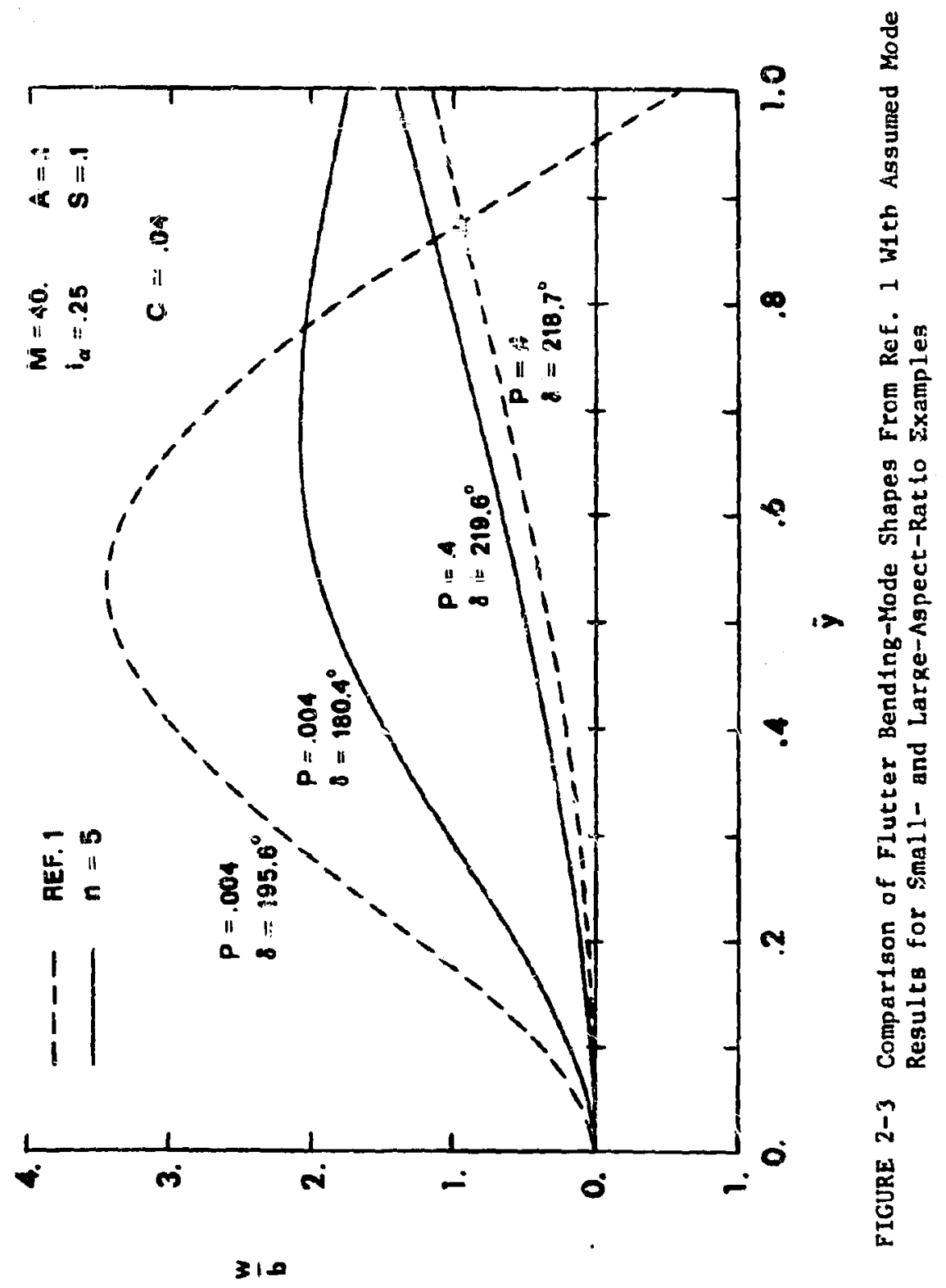


Chapter III

DEVELOPMENT OF A GENERAL SYSTEM OF EQUATIONS FOR VERTICAL BENDING/CHORDWLSE BENDING/TORSION (VCT)

\section{A. Introduction}

Chapter II includes drag loads in transverse bending and torsion but allows no chordwise deflections. In other words, it nssumes infinitely large bending rigidity in the fore-and-aft direction. In chis chapter we consider chordwise bending, which is a more complete model of the true physical situation.

A review of the literature for work conrerning $i C T$ motion of slender cantilever beams led to the field of hingeless helicopter rocor stability analysis. The structural modeling of a hingeless rotor ts essentlally the same as desired here, except that the wing has no rotational velpcity. Accordingly. If an adequate model for a hingeless rotor can be found, it can be adapted foi the cantllever wing by removing the inertial and cantrifugal tension effects arising from rotation. Niucl! of the work pursued in the hingeless rotor field includes simplifications which either eliminate or restrict one of the three types of deformation, of ten torslon, in an effort to reduce complexity. This leaves a tolativeig small body of work that treats the full elast le $\checkmark$

protlera. A well-known system of linear partial differential equations for coupled elastic corsion and bending of twisted nonuniform rotor blades is that developed by Houbolt and Brooks (Ref. 6); the initidi effort to develop a VCT svstem for the cantilever wing centered on adapting this formulation. In the course of this work, however, the elastic coupling terms were found to be insufficient to account for the 
drag loads in transverse bending and corsion embodied by the terms containing $M_{z}$ in $(2-1)$. It then became apparent that this drag coupling is actually a nonlineat structural bending-ioralos effect and that the new system of equations to be developed should, of mecessity, recain all nonlinear elastic coupling terms having the same order of importance as these drag coupling terws.

Further search led to the gystem of nonlinear equations fer culsted nonuniform rotor blades derived by Hodses and Dowell (Ref. 7). Thls work involves development of a more coaplece strain-displacement relaclon than that of Ref. 6. Which la necessary to obeala the elastic bending-torsion coupling cerws that produce the desired drag coupling effect. The equations in Ref. 7 are valid tor atralghe, sender.

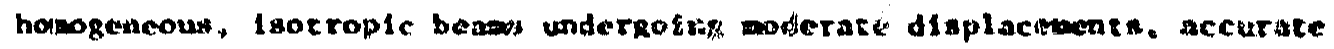
to second order in the sense of rentriztion that squares of bending slopes, cular. and atrfoll chord and chlckness divided by wink wealapan are sanll wh raspect to unity.

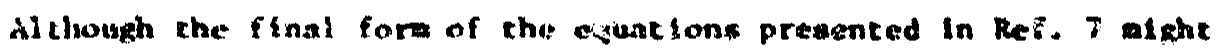
appant so be tmediately adaptabic to the present case by settag the rotor rotation frequency to zero and removing the effecs of pretwist. chis is not entirely true. One laportant assamption by Hodges and Dowell requlted that the rat lo between the cransverge and chordulse beam bending stiffnesses be a quancity of order one. Whereas this is a standard feacure of helicopter blade construction. It usually does not hold for conventlonal aircraft wings of any aspect ratio. As a result, the Ref. 7 derivation has been carefully retraced for the specific case of a nonrotating cantilever wing having arbitrary bending stiffness ratio. The development is outlined in the following section. 


\section{B. Development of the Nonlinear Equations of Motion}

Derivation of the VCT model for the uniform cantilever wing is presented here in abbreviated form. Important modifications of the Ref. 7 derivation are emphasized, but duplicate manipulations are only briefly described. The notation and coordinate system for the cantilever wing is used exclusively.

The basic ordering scheme presented in equation (4) of Ref. 7 is retained. One exception is that spanwise warping of the cross section due to twtsting, represented by a warp displacement function which is a solution of the Laplace equation over the cross section, is entirely neglected here. This assumption is made on the premise that a typical afreratt wing airfoil section would have a sufficiently small tirickness that warping effects would be negligible within the second-order framework. The ordering scheme is applied to the energy expressions encountered in the variational derivation of the equations, to determine which Eerms should be retained and which discarded.

The nonlinear strafn displacement relacions developed in Ref. 7 have been carefully examined in the context of the present problem, and they are found to apply without modifications. These relations are derived from an exact transformation between the deformed and undeformed coordinate sytems and originate from the classical. definition of strain of Novozhilov (Ref. 19) which is based upon increments in the deformed coordinates. After approximation to second order consistent with all assumptions, the final form in terms of engineering strain and in the present notation is 


$$
(3-1)\left\{\begin{array}{c}
\varepsilon_{y y}=u_{y}^{\prime}+\frac{u_{x}^{\prime 2}}{2}+\frac{u_{z}^{\prime 2}}{2}-u_{x}^{\prime \prime}[\xi \cos \theta+n \sin \theta) \\
-u_{z}^{\prime \prime}(-\varepsilon \sin \phi+n \cos \phi) \\
\varepsilon_{y \xi}=n \phi^{\prime} \\
\varepsilon_{y \eta}=-\varepsilon, \phi^{\prime}
\end{array}\right.
$$

These can be recognized as identical to equations (24), (25), (26), and (27) of Ref. T after the warp function and pretulst angle have been eliminated. The displacements $u_{x} \cdot u_{y}, u_{z}$ of the elaste axis and $t^{\text {t }}$ principal coordinates $\xi$. $\eta$ of the cross sectlon are lllustrated in Figure 3.1.

Development of the equat lors us ing the indirect caiculus of variations to based upon Hamilton's principle, which may be stated in che form

$$
\int_{t_{1}}^{t_{2}}[\delta(u-T) \cdots \delta(w) d t=0
$$

The equacions art obtatned by combinine expressions for the first variation of strain energy $\delta ! l$. kinetic energy $\delta T$. and virtual work of extermal forces $s(!)$.

The first variation of strain energy in appropriace form for the standard Euler-Bernoulli beam unfaxial stress-ytrain relacionship $1 ;$

$$
\delta \omega=\int_{0}^{\ell}{ }_{\text {area }} \delta\left(\sigma_{y y} \delta \varepsilon_{y y}+\sigma_{y} \delta_{y \varepsilon} \delta \varepsilon_{y}+\sigma_{y n} \delta \varepsilon_{y n}\right) d \varepsilon d n d y
$$

The first varlation of the engineering strains, expressed in teras of displacements, is 
$(3-4)\left\{\begin{aligned} \delta \varepsilon_{y y}= & \delta u_{y}^{\prime}+u_{x}^{\prime} \delta u_{x}^{\prime}+u_{z}^{\prime} \delta u_{z}^{\prime} \\ & -[\xi \cos \phi+\eta \sin \phi]\left(\delta u_{x}^{\prime \prime}-u_{z}^{\prime \prime} \delta \phi\right) \\ & -\left[\xi \sin \phi^{\prime}+\eta \cos \phi\right]\left(\delta u_{z}^{\prime \prime}+u_{x}^{\prime \prime} \delta \phi\right) \\ \delta \varepsilon_{y}= & \eta \delta \phi^{\prime} \\ \delta \varepsilon_{y \eta}= & -\xi \delta \phi^{\prime}\end{aligned}\right.$

The stresses are simply

$(3-5)\left\{\begin{array}{l}\sigma_{y y}=E \varepsilon_{y y} \\ \sigma_{y \xi}=G \varepsilon_{y \xi} \\ \sigma_{y \eta}+G \varepsilon_{y \eta}\end{array}\right.$

Substituting (3-1), (3-4), (3-5) into (3-3) yields

$(3-6)\left\{\begin{aligned} \delta U= & \int_{0}^{l} \underset{\text { area }}{\int}\left\{E\left[u_{y}^{\prime}+\frac{u_{x}^{\prime 2}}{2}+\frac{u_{z}^{\prime 2}}{2}-u_{x}^{\prime \prime}(\xi \cos \phi+n \sin \phi)\right.\right. \\ & \left.-u_{z}^{\prime \prime}(-\xi \sin \phi+n \cos \phi)\right]\left[\delta u_{y}^{\prime}+u_{x}^{\prime} \delta u_{x}^{\prime}+u_{z}^{\prime} \delta u_{z}^{\prime}\right. \\ & -(\xi \cos \phi+n \sin \phi)\left(\delta u_{x}^{\prime \prime}-u_{z}^{\prime \prime} \delta \phi\right) \\ & \left.-(-\xi \sin \phi+n \cos \phi)\left(\delta u_{z}^{\prime \prime}+u_{x}^{\prime \prime} \delta \phi\right)\right] \\ & \left.+G\left[n^{2} \phi^{\prime} \delta \phi^{\prime}+\xi^{2} \phi^{\prime} \delta \phi^{\prime}\right]\right\} d n d \xi_{z} d y\end{aligned}\right.$

Rearrangement of (3-6) by grouping of terms having the same virtual displacements leads to 


$$
\begin{aligned}
\delta u= & \int_{0}^{l}\left[v_{y}\left(\delta u_{y}^{\prime}+u_{x}^{\prime} \delta u_{x}+u_{z}^{\prime} \delta u_{z}^{\prime \prime}\right)+s_{y} \delta \phi^{\prime}\right. \\
& +\left[-M_{z} \cos \phi+M_{x} \sin \phi\right]\left(\delta u_{x}^{\prime \prime}-u_{z}^{\prime \prime} \delta \phi\right) \\
& \left.+\left[M_{z} \sin \phi+M_{x} \cos \phi\right]\left(\delta u_{z}^{\prime \prime}+u_{x}^{\prime \prime} \delta \phi\right)\right] d y
\end{aligned}
$$

The stress resultants and moments formed in (3-7), which act on the deformed wing as 111 ustrated in Fig. 3.2, are defined as follows

$$
\begin{aligned}
\mathrm{V}_{\mathrm{y}} & \equiv \underset{\text { area }}{\int} \sigma_{\mathrm{yy}} \mathrm{d} E_{\mathrm{d}} \mathrm{d} n \\
& =\mathrm{EA}_{\mathrm{w}}\left\{u_{y}^{\prime}+\frac{u_{x}^{\prime 2}}{2}+\frac{u_{z}^{\prime 2}}{2}-e_{A}\left(u_{x}^{\prime \prime} \cos \phi-u_{z}^{\prime \prime} \sin \phi\right)\right\}
\end{aligned}
$$

$$
\begin{aligned}
& \text { where } \mathrm{A}_{\mathrm{w}} \mathrm{e}_{\mathrm{A}} \equiv \underset{\text { area }}{\int J} \xi \mathrm{d} \xi \mathrm{d} \eta \\
& \text { and } \underset{j}{f} \text { it if } A r=0 \text {, by definition } \\
& M_{z} \equiv \underset{\text { area }}{\iint} \xi a_{y y} d \xi_{j} d n \\
& =E I_{z}\left(-u_{x}^{\prime \prime} \cos +u_{z}^{\prime \prime} \sin \right)+E A_{w} e_{A}\left(u_{y}^{\prime}+\frac{u_{x}^{\prime 2}}{2}+\frac{u_{z}^{\prime 2}}{2}\right)
\end{aligned}
$$

where $\mathbf{I}_{z} \equiv \underset{\text { area }}{\int J} \xi^{2} \mathrm{~d} \xi_{j} \mathrm{~d} n$

$$
\begin{aligned}
& \text { and } \underset{\text { area }}{\int} \xi \eta \mathrm{d} \xi \mathrm{d} \eta \approx 0 \\
& M_{x} \equiv-\underset{\text { orea }}{\iint} n \sigma_{y y} d \delta_{d} d n \\
& =E I_{x}\left(u_{x}^{\prime \prime} \sin \phi+u_{z}^{\prime \prime} \cos \phi\right)
\end{aligned}
$$

where $I_{x} \equiv \underset{\text { area }}{\pi} \eta^{2} \mathrm{~d} \xi_{\mathrm{s}} \mathrm{d} n$ 
$(3-11)$

$$
\text { Sy } \equiv \underset{\text { area }}{S}\left(-\xi \sigma_{y \eta}+n \sigma_{x \xi}\right) d \xi d \eta
$$

$$
=G I_{d} \phi^{\prime}
$$

$$
\text { where } \mathbf{I}_{\mathrm{d}} \equiv \underset{\text { area }}{\int}\left\langle\xi^{2}+\eta^{2}\right) d \xi d r_{1}
$$

At this point in the development the moments of Inertia $I_{x}, I_{z}$ are first introduced. Herediter, it must be recognized that $I_{z}$ may assume values much larger than $I_{x}$. The terms containing the quantity $e_{A}$, which measures the offset of the tenslle axis (area centroid) from the clastic axis, will be dropped. The basis for this simplification is that, in the final modal formulation of the equations, the retention of $e_{A}$ terms ultimately leads to a dimensionless parametar, $\frac{\overline{E A}_{w} e_{A}}{E I}$, which appears only as a small quantity added or subtracted with unity. Since it will not significantly influence dynamic stability, hereafter $e_{A_{A}}=0$ will be assumed.

After appropriate integrations by parts within equation (3-7), the final form for Lne first variation of strain energy, including boundary terms, is

$$
\begin{aligned}
\delta U= & \int_{0}^{\ell}\left\{\left[-\left(v_{y} u_{x}^{\prime}\right)^{\prime}+\left(-M_{z} \cos \phi+M_{x} \sin \phi\right)^{\prime \prime}\right] \delta u_{x}\right. \\
& -\left(v_{y}\right)^{\prime} \delta u_{y}+\left[-\left(v_{y} u_{z}^{\prime}\right)^{\prime}+\left(M_{z} \sin \phi+M_{x} \cos \phi\right)^{\prime \prime}\right] \delta u_{z} \\
& \left.+\left[-\left(S_{y}\right)^{\prime}-u_{z}^{\prime \prime}\left(-M_{z} \cos \phi+M_{x} \sin \phi\right)+u_{x}^{\prime \prime}\left(M_{z} \sin \phi+M_{x} \cos \phi\right)\right] \delta \phi\right\} d y \\
& +\left.v_{y} \delta u_{y}\right|_{0} ^{\ell}+\left.u_{x}^{\prime} v_{y} \delta u_{x}\right|_{0} ^{l}+\left.u_{z}^{\prime} v_{y} \delta u_{z}\right|_{0} ^{l}+\left.s_{y} \delta \phi\right|_{0} ^{\ell} \\
& +\left.\left[-M_{z} \cos \phi+M_{x} \sin \phi\right] \delta u_{x}^{\prime}\right|_{0} ^{l}-\left.\left[-M_{z} \cos \phi+M_{x} \sin \phi\right]^{\prime} \delta u_{x}\right|_{0} ^{\ell} \\
& +\left[M_{z} \sin \phi+\left.M_{x} \cos \phi j \delta u_{z}^{\prime}\right|_{0} ^{\ell}-\left.\left[M_{z} \sin \phi+M_{x} \cos \phi\right]^{\prime} \delta u_{z}\right|_{0} ^{\ell}\right.
\end{aligned}
$$


Formulation of the first variation of the kinetic energy is greatly simplified relative to the Ref. 7 derivation because there are no inertial effects introduced by rotation of the helicopter blade. Since the procedure is straightforward and well described in Ref. 7 , the detalls of forming the kinetic energy il terms of displacement velocities, taking the first variation, Integrating by parts over time, and expressing the resulting form of $\delta T$ in terms of time derivatives of $u_{x}, u_{y}$, $u_{z}$, and $\phi$ are omitted here. After the ordering scheme has been applied, the form of the first varlation of kinetlc energy, with terms retained to second order and corresponding to equation (52) of Ref. 7 , is

$$
\begin{aligned}
\delta T \equiv & \int_{0}^{l} \underset{\text { area }}{J} \rho_{s}\left[-\left[\ddot{u}_{x}+\phi(-\varepsilon \sin \phi+n \cos \phi)\right] \delta u_{x}\right. \\
& -\left[\ddot{u}_{z}-\phi(\xi \cos \phi+n \sin \phi)\right] \delta u_{z}
\end{aligned}
$$

$$
\begin{aligned}
& -\left[\ddot{u}_{x}(-\xi \sin \phi+\zeta \cos \phi)-\ddot{u}_{z}(\xi \cos \phi+n \sin \phi)\right] \delta \phi \\
& \left.-\ddot{\phi}\left[(-\xi \sin \phi+n \cos \phi)^{2}+(\xi \cos \phi+n \sin \phi)^{2}\right] \delta \phi\right] d \eta d \xi d y
\end{aligned}
$$

As noced in Ref. 7, the last term in this expression is by definition a third urder term, but it is retained in order to include torsional inertia in the torsion equation.

The final form of the first varlation of kinetic energy is obtained by integration over the sectional area, which leads to the following definitions: 


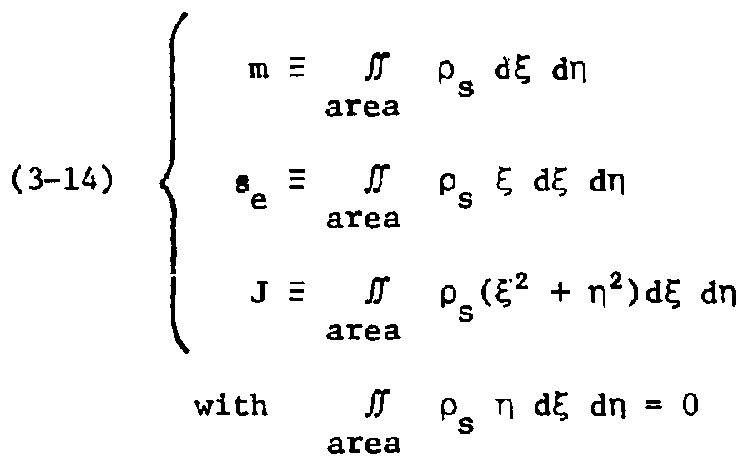

The final form is

(3-15)

$$
\delta T \approx \int_{0}^{l}\left\{\left(-m u_{x}+s e^{\phi s \ln \phi) \delta u_{x}+\left(-m \ddot{u}_{z}+s\right.} e^{\phi \cos \phi) \delta u_{z}}\right.\right.
$$

$$
\left.+\left[s_{e} \ddot{u}_{x} \sin \phi+s_{e} \ddot{u}_{z} \cos \phi-J \phi\right] \delta \phi\right\} d y
$$

The virtual work of the applied loads is

$(3-16) \quad \delta u^{\prime \prime}=\int_{0}^{l}\left(F_{x} \delta u_{x}+F_{z} \delta u_{z}+m_{y} \delta \phi\right) d y$

Clearly the drag loads will now enter the equations of motion in the same manner as the lifting airloads, in contrast wath (2-1), since $\mathrm{F}_{\mathrm{x}}$ and $\mathrm{F}_{2}$ will consist of lift and drag force components.

Application of Hamilton's principle using (3-12), (3-15), and (3-16), together with $(3-8),(3-9),(3-10)$, and (3-11), results in the following quantities being required to vanish:

$\frac{\delta \mathrm{u}}{\mathrm{y}}$ terms

$(3-17 a)-\left[E A_{w}\left(u_{y}^{\prime}+\frac{u_{x}^{\prime 2}}{2}+\frac{u_{x}^{\prime 2}}{2}\right)\right]^{\prime} \equiv T^{\prime}=0$ 
$\underline{\delta u}$ terms

$$
\left[-E I_{z}\left(-u_{x}^{\prime \prime} \cos \phi+u_{z}^{\prime \prime} \sin \phi\right) \cos \phi\right.
$$

$\left.(3-17 b)+E I_{x}\left(u_{x}^{\prime \prime} \sin \phi+u_{z}^{\prime \prime} \cos \phi\right) \sin \phi\right)^{\prime \prime}+m \ddot{x}_{x}$

$$
-s_{e} \phi \sin \phi=0
$$

$\underline{\delta}$ terms

$$
\left\{E I_{z}\left(-u_{x}^{\prime \prime} \cos \phi+u_{z}^{\prime \prime} \sin \phi\right) \sin \phi\right.
$$

$(3-17 c)$

$$
\begin{aligned}
& +E I_{x}\left(u_{x}^{\prime \prime} \sin \phi+u_{z}^{\prime \prime} \cos \phi\right) \cos \phi j^{\prime \prime}+m_{z} \\
& -s_{e} \phi \cos \phi-F_{z}=0
\end{aligned}
$$

\section{Sф terms}

$(3-17 d)$

$$
\begin{aligned}
& -\left(G I_{d} \phi^{\prime}\right)^{\prime}-u_{z}^{\prime \prime}\left[-E I_{z}\left(-u_{x}^{\prime \prime} \cos \phi+u_{z}^{\prime \prime} \sin \phi\right) \cos \phi\right. \\
& \left.+E I_{x}\left(u_{x}^{\prime \prime} \sin \phi+u_{z}^{\prime \prime} \cos \phi\right) \sin \phi\right] \\
& +u_{x}^{\prime \prime}\left[E I_{z}\left(-u_{x}^{\prime \prime} \cos \phi+u_{z}^{\prime \prime} \sin \phi\right) \sin \phi+E I_{x}\left(u_{x}^{\prime \prime} \sin \phi+u_{z}^{\prime \prime} \cos \phi\right) \cos \phi\right] \\
& -\varepsilon_{e} \ddot{u}_{x} \sin \phi-s_{e} \ddot{u}_{z} \cos \phi+J \phi-M_{y}=0
\end{aligned}
$$

In (3-17a), since it is known that for the nonrotating contilever wing the spanwise tension $T$ will be zero everywhere, the expression can be integrated leaving

(3-18) $\quad u_{y}^{\prime}+\frac{u_{x}^{\prime 2}}{2}+\frac{u_{z}^{\prime 2}}{2}=0$ 
(true only with $\mathbf{e}_{A}=0$ ). This constraint indicates that the only manner by which axial deflections $u_{y}$ enter the problem is by the purely geometric dependence on $u_{x}$ and $u_{z}$ due to shortening induced by the lateral deflections.

In replacing sin $\phi$ and $\cos \phi$ by small angle approximations, it would be consistent with the ordering scheme to approximate the cosine by unity. Due to the possibility of a large ratio of bending stiffnesses, which can result in $u_{z}$ being large compared with $u_{x}$, it was found necessary to keep the second-order approximation of the cosine at this sten. This is done to derive properly certain elastic coupling terms involving $u_{z}$ and $u_{x}$, while maintaining symmetry in the inertial and stiffness matrices of the final matrix equations.

Substitution of

(3-19)

$$
\cos \phi \approx 1-\frac{\phi^{2}}{2}
$$$$
\sin \phi \approx \phi
$$

into (3-17) leads to

(3-20a)

$$
\begin{aligned}
{\left[-E_{z}\left(-u_{x}^{\prime \prime}\left(1-\phi^{2}\right)\right.\right.} & \left.\left.+u_{z}^{\prime \prime} \phi\right)+E I_{x}\left(u_{x}^{\prime \prime} \phi^{2}+u^{\prime \prime} \phi\right)\right] " \\
& +\operatorname{mï}_{x}-s_{e} \ddot{\phi} \phi=F_{x}
\end{aligned}
$$

$(3-20 b)$

$$
\begin{aligned}
{\left[E I_{z}\left(-u_{x}^{\prime \prime}+u_{z}^{\prime \prime} \phi\right) \phi\right.} & \left.+E I_{x}\left(u_{x}^{\prime \prime} \phi+u_{z}^{\prime \prime}\left(1-\phi^{2}\right)\right)\right] " \\
& +m \ddot{u}_{z}^{\prime}-s_{e} \phi=F_{z}
\end{aligned}
$$




$$
\begin{aligned}
&-\left(G I_{d} \phi^{\prime}\right)^{\prime}-u_{z}^{\prime \prime}\left[-E I_{z}\left(-u_{x}^{\prime \prime}\left(1-\phi^{2}\right)+u_{z}^{\prime \prime} \phi\right)+E I_{x}\left(u_{x}^{\prime \prime} \phi^{2}+u_{z}^{\prime \prime} \phi\right)\right] \\
&(3-20 c)-u_{x}^{\prime \prime}\left[E I_{z}\left(-u_{x}^{\prime \prime} \phi+u_{z}^{\prime \prime} \phi^{2}\right)+E I_{x}\left(u_{x}^{\prime \prime} \phi+u_{z}^{\prime \prime}\left(1-\phi^{2}\right)\right)\right] \\
&-s_{e} u_{x} \phi-s_{e} \ddot{u}_{z}+J \phi=m_{y}
\end{aligned}
$$

Reorganization of terms and introduction of the displacements w and $v$ for $u_{z}$ and $u_{x}$, respectively, gives

$(3-21 a)$

$$
\left\{\left[E I_{z} \phi^{2}+E I_{x}\left(1-\phi^{2}\right)\right] w^{\prime \prime}-\left(E I_{z}-E I_{x}\right) v^{\prime \prime} \phi\right\}^{\prime \prime}
$$

$(3-21 b)$

$$
\left\{-\left(E I_{z}-E I_{x}\right) w^{\prime \prime} \phi+\left[E I_{z}\left(1-\phi^{2}\right)+\phi^{2} E I_{x}\right] v^{\prime \prime}\right\} "
$$

$(3-21 c)$

$$
\begin{aligned}
& +m \ddot{v}-s_{e} \phi \phi=F_{x} \\
& G I_{d} \phi^{\prime \prime}-\left(E I_{z}-E I_{x}\right)\left[\left(w^{\prime \prime 2}-v^{\prime \prime 2}\right) \phi-v^{\prime \prime} w^{\prime \prime}\left(1-\underline{2 \phi^{2}}\right)\right] \\
& +\underline{s}_{e^{\ddot{v} \phi}}+s_{e^{\ddot{w}}-J \ddot{\phi}+m_{y}=0}
\end{aligned}
$$

The underlined terms are reasoned to represent higher-order effects and are dropped. In the case of the inertial terms coupling chordwise bending and torsion through the offset $s_{e}$, this is a third-order effect relative to terms like $m \ddot{v}$. The final form of the three-DOF system of equations, with some regrouping, is

$(3-22 a)$

$$
\left\{E I_{x} w^{\prime \prime}-\left(E I_{z}-E I_{x}\right)\left(v " \phi-\underline{w " \phi^{2}}\right)\right\} "
$$

$$
+m \ddot{w}-s_{e} \phi=F_{z}
$$


$(3-22 b)$

$$
\left[E I_{z} v^{\prime \prime}-\left(E T_{z}-E I_{x}\right)\left(w^{\prime \prime} \phi+\underline{\underline{v} " \phi^{2}}\right)\right\} "+m \ddot{v}=F_{x}
$$

$$
\begin{gathered}
G I_{d} \phi^{\prime \prime}-\left(E I_{z}-E I_{x}\right)\left[\left(\underline{\left(w^{\prime \prime 2}\right.}-\underline{v^{\prime \prime 2}}\right) \phi-v^{\prime \prime} w^{\prime \prime}\right] \\
+s_{e} \ddot{w}=J \dot{w}+m_{y}=0
\end{gathered}
$$

This system is elastically and inertially self adjoint, which assures that the stiffness and inertia matrices eventually assembled during modal anailysis will be symetric. The tarms containing $\left(E I_{z}-E I_{x}\right)$ represent the nonlinear couping between the torsion and bending degrees of frcedom; all remaining stiffness, inertial, and applied-load terms represent the same familiar forms encountered in linear beam theory. Terns in (3-22) which are underlined do not appear in the Ref. 7 equations and are retained here as a result of the absence of a restriction on the bending stiffness ratio $\mathrm{EI}_{z} / \mathrm{EI} \mathrm{I}_{\mathrm{X}} \cdot$ Strictly speaking, when this ratio is large compared with unity, the single underlined terms will increase in relative importance while the double underlined terms are negligible in magnitude.

The nonlinear equations of motion (3-22) are next adapted to permit analysis of stability aiout a steady-state deflected position due to an equilibrium lifting flight condition, which could be leve1 flight or a steady pullout at a high load factor. Small time-dependent perturbations about the equilibrium operating condition are introduced by expressing the deflections $w, v$, and $\phi$ in terms of steady-state equilibrium deflections $w_{0}, v_{0}$, and $\phi_{0}$ and small unsteady perturbation quantities $w_{1}, v_{1}$, and $\phi_{1}$ : 
$(3-23) \quad\left\{\begin{array}{l}w(t)=w_{0}+w_{1}(t) \\ v(t)=v_{0}+v_{1}(t) \\ \phi(t)=\phi_{0}+\phi_{1}(t)\end{array}\right.$

First, the steady equilibrium deflections only are substituted into (3-22) to abtain a nonlinear system of equations for the equilibrium solution. The resulting nonlinear steady sys:em is

$$
\begin{aligned}
& \text { (3-24a) } \quad\left\{E I_{x} w_{0}^{\prime \prime}-\left(E I_{z}-E I_{x}\right)\left(v_{0}^{\prime \prime} \phi_{0}-w_{0}^{\prime \prime} \phi_{0}^{2}\right)\right\}^{\prime \prime}=F_{z_{0}} \\
& \text { (3-24b) } \quad\left\{E I_{z} v_{0}^{\prime \prime}-\left(E I_{z}-E I_{x}\right)\left(w_{o}^{\prime \prime} \phi_{0}+v_{o}^{\prime \prime} \phi_{0}^{2}\right)\right\} "=F_{x_{0}} \\
& (3-24 c) \quad G I_{d} \phi_{0}^{\prime \prime}-\left(E I_{z}-E I_{x}\right)\left[\left(w_{0}^{\prime \prime 2}-v_{0}^{\prime \prime 2}\right) \phi_{0}-v_{o}^{\prime \prime} w_{0}^{\prime \prime}\right]+m_{y_{0}}=0
\end{aligned}
$$

Appropriate steady lifting aerodynamic loads are inserted for $L_{w_{0}}$ and $M_{\phi_{0}}$, and the assumed steady drag, which entered equations (2-1) in an entirely different manner, is introduced through $\mathrm{L}_{\mathrm{v}_{0}}$.

Next a linearized system of equations in the time dependent small perturbation deflections is obtained by substituting (3-23) into (3-22), subtracting the nonlinear equilibrium equations, and discarding products of the perturbation quantities. The linearized unsteady equations of motion are

$$
\begin{gathered}
\left\{E I_{x} w_{1}^{\prime \prime}-\left(E I_{z}-E I_{x}\right)\left[\phi_{0} v_{1}^{\prime \prime}+\phi_{1} v_{0}^{\prime \prime}-\phi_{0}^{2} w_{1}^{\prime \prime}-2 \phi_{0} w_{0}^{\prime \prime} \phi_{1}\right]\right\} " \\
+m \ddot{w}_{1}-s_{e} \phi_{1}-F_{z_{1}}=0
\end{gathered}
$$


$(3-25 b)$

$$
\begin{aligned}
& \left\{E I_{z} v_{1}^{\prime \prime}-\left(E I_{z}-E I_{x}\right)\left[\phi_{0} w_{1}^{\prime \prime}+w_{o}^{\prime \prime} \phi+\phi_{0}^{2} v_{1}+2 \phi_{0} v_{o}^{\prime \prime} \phi_{1}\right]\right\} " \\
& \quad+m \ddot{v}_{1}-E_{x_{1}}=0 \\
& G I_{d} \phi_{1}^{\prime \prime}-\left(E I_{z}-E I_{x}\right)\left\{\left[2 w_{0}^{\prime \prime} w_{1}^{\prime \prime}-2 v_{0}^{\prime \prime} v_{1}^{\prime \prime}\right] \phi_{0}+\left[w_{0}^{\prime \prime 2}-v_{0}^{\prime \prime 2}\right] \phi_{1}\right. \\
& \left.-w_{o}^{\prime \prime} v_{1}^{\prime \prime}-v_{o}^{\prime \prime} w_{1}^{\prime \prime}\right\}+s_{e}^{\ddot{w}_{1}}-J \phi_{1}+m_{y_{I}}=0
\end{aligned}
$$

$(3-25 c)$

Analysis of stability with this system can be done with the standard techniques for linear systems, but first the coefficients must be found for a given equilibrium flight condition by solving (3-24). The loads appearing in this equation, including the chordwise forces, must be expressed as linear functions of perturbation displacements $w_{1}$ and $\phi_{1}$. No dependence of aeroaynamic loads on the fore-and-aft motion $v_{1}$ will be considered in this analysis.

C. Comparison of the VT and VCT Models

The VCT model (3-24) and (3-25), although aifferent in appearance from the VT model (2-1), in fact reduces to the same form when $\tau+\infty$ and the steady lift is zero. To illustrate this, first imagine that the steady loads applied to (3-24) are $(3-26) \quad\left\{\begin{array}{l}F_{z_{0}}=0 \\ m_{y_{0}}=0 \\ F_{x_{0}}=D\end{array}\right.$ 
where $D$ is the same assumed drag force, constant in magnitude and direction along the span, as is considered in (2-2). The solution to (3-24) immediately gives $w_{0}=\phi_{0}=0$, leaving just the chordwise bending equation as

$$
\begin{aligned}
& \text { (3-27) } \quad \text { EI }_{z} v_{0}^{\prime \prime \prime \prime}=D \\
& \text { Substitution of } \tau E I_{x} \text { for } E_{z} \text { in (3-25) together with } \\
& w_{0}=\phi_{0}=0 \text { gives } \\
& \text { (3-28a) } \quad E_{x} w_{1}^{\prime \prime \prime \prime}-(\tau-1) E I_{x}\left(\phi_{1} v_{0}^{\prime \prime}\right) "+m \ddot{w}_{1}-s_{e} \phi_{1}-F_{z_{1}}=0 \\
& \text { (3-28b) TEI } x_{1}^{\prime \prime \prime \prime}+\ddot{m}_{1}-F_{x_{1}}=0 \\
& \text { (3-28c) } \quad \mathrm{GI}_{d} \phi_{1}^{\prime \prime}-(\tau-1) E I_{x}\left[-v_{0}^{\prime \prime 2} \phi_{1}-v_{0}^{\prime \prime} w_{1}^{\prime \prime}\right]+s_{e} \ddot{w}_{1}-J \phi_{1}+m_{y_{1}}=0
\end{aligned}
$$

The equation for $v_{1}$ uncouples and can be disregarded. Integration of (3-27) twice, using the zero shear and moment boundary conditions at the free end, gives

$$
\tau E I_{x} v_{0}^{\prime \prime}=D \stackrel{1}{2}(l-y)^{2}
$$

(3-29)

$$
=-\mathbf{M}_{\mathbf{Z}}
$$

where (2-2) has been used. Substitution of (3-29) into equations $(3-28 a, c)$ yields

(3-30a) $\quad I_{x} x_{1}^{\prime \prime \prime}+\frac{\tau-1}{\tau}\left(M_{z} \phi_{1}\right)^{\prime \prime}+\ddot{m}_{1}-s_{e_{1}}-F_{z_{1}}=0$ 
$(3-30 b)$

$$
G I_{d} \phi_{1}^{\prime \prime}-\frac{\tau-1}{\tau} M_{2}\left(w_{1}^{\prime \prime \prime}+v_{0}^{\prime \prime} \phi_{1}\right)+s e_{e_{1}}^{\ddot{w}_{1}}-J \phi_{1}+m_{y_{1}}+0
$$

Finally, requiring that $\tau \rightarrow \infty$, with the consequent vanishing of $v_{0}$, causes $(3-30)$ to reduce exactly to $(2-1)$.

For the more general case or̈ a steady lifting condition characterized by $w_{0} \neq 0$ and $\phi_{0} \neq 0,(3-24)$ and $(3-25)$ can still be cast into a form similar to $(2-1)$, in which the $v_{1}$ displacements become dependent upon $w_{1}$ and $\phi_{1}$ since $\tau+\infty$. First, the nonlinear steady equations (3-24) are rewritten with TEI $x$ replacing $E I_{z}$ together with some rearrangement of terms to give

$$
\begin{aligned}
& \left.(3-31 a) \quad E_{x} w_{0}^{\prime \prime \prime}-\tau E I_{x} \frac{T-1}{\tau}\left[v_{0}^{\prime \prime}-w_{0}^{\prime \prime} \phi_{0}\right) \phi_{0}\right] "=F_{z_{0}} \\
& (3-31 b) \quad T E I_{x}\left[v_{0}^{\prime \prime}-\frac{\tau-1}{\tau}\left(w_{0}^{\prime \prime} \phi_{0}+v_{0}^{\prime \prime} \phi_{0}^{2}\right)\right]=F_{x_{0}} \\
& (3-31 c) \quad G I_{d} \phi_{0}^{\prime \prime}-\frac{\tau-1}{\tau} \tau E I_{x}\left[\left(w_{0}^{\prime \prime} \phi_{0}-v_{0}^{\prime \prime}\right) w_{0}^{\prime \prime}-v_{0}^{\prime \prime 2} \phi_{0}\right]+m_{y_{0}}=0
\end{aligned}
$$

Again, as $\tau \rightarrow \infty$ the elastic bending curvature about the $n$ principal axis (Fig. 3.1) of the airfoil section should go to zero. But now, the spction is displaced to a position fixed by the deflections $w_{0}, v_{0}$, and $\phi_{0}$ of its elastic axis, and tise true elastic curvature about the $\eta$ axis is now recognized as $v_{0}^{\prime \prime}-w_{0}^{\prime \prime} \phi_{o}$.

In equation (3-31b), which is the fore-and-aft equilibrium equation, $22 \tau$ becomes large and $v_{0}$ becomes small, the texm $v_{0}^{\prime \prime} \phi_{0}^{2}$ is of higher order and can be neglected relative to $v_{0}^{\prime \prime}$. Removing this term and letting $\frac{\tau-1}{\tau} \rightarrow 1$ leaves 
(3-32)

$$
\operatorname{TEI_{x}}\left[v_{0}^{\prime \prime}-w_{0}^{\prime \prime}\right]_{0}^{\prime \prime}=F_{x_{0}}
$$

Integrating this equation twice, using the shear and moment boundarv conditions on $v_{0}$ and $w_{0}$ at the free end, leads to

$$
\operatorname{TEI_{X}}\left[v_{0}^{\prime \prime}-w_{0}^{\prime \prime} \omega_{0}\right]=F_{x_{0}}:(R-v)^{2} \equiv-M_{z_{0}}
$$

Here it is assumed for convenience that $F_{X_{F}}$ is constant along the span; of course the integrations could as well be twerformed for an: knuwn spanwise variation of $F_{x_{0}} \cdot M_{z_{0}}$ is simflar to $M_{z}$ of (3-29). except that it represents the bending-torston coupling effect upon the moment-curvature relation. As $t+\infty$ the quantity $\left(v_{0}^{\prime \prime}-w_{0}^{\prime \prime}\right.$ must go to zero according to (3-33). In the limit the $v_{0}$ deflection becomes dependent upon $w_{0}$ ano ${ }_{0}$ as a result of bending-torslon coupling.

The two remaining findepesdent equations in (3-31) are already arranged so that the coupled curvature quantitv in (3-33) can be recognized. Sutstitution for tinis quantity laads to

$(3-34)$

$$
E I w_{0}^{6 \cdots+\cdots}+\frac{t-1}{\tau}\left[M_{z_{0}} \phi_{0}\right]^{\prime \prime}=F_{z^{\circ}}
$$

$$
G I_{d} \phi_{0}^{\prime \prime}-\frac{T-1}{\tau}\left[M_{z_{0}} w_{0}^{\prime \prime}-v_{0}^{\prime \prime} \omega_{0}\right]+m_{0}=0
$$

As $T \rightarrow \infty$, with $v_{0}^{\prime \prime}$ recognized as a higher-order term. the nonlinear steady equilibrium equations finally become

$$
\text { (3-35a) EIw } w_{0}^{\prime \prime \prime}+\left[M_{z_{0}} \emptyset_{0}\right]^{\prime \prime}-F_{z_{0}}=0
$$


$(3-35 b)$

$$
G I_{d} \phi_{0}^{\prime \prime}-M_{z_{0}} w_{0}^{\prime \prime}+m_{y_{0}}=0
$$

These resemble (2-1) with dynamic terms removed.

A Inal exercise is to demonstrate that the linearized unsteady perturbation equations (3-25) also reduce in a systematic fashton to a VT system when $\tau+\infty$. When (3-25) are rearranged with $T E I_{x}$ replacing $\mathrm{EI}_{2}$ and the combination of steady deflections in (3-33) replaced by $M_{z_{0}}$, the lineartzed system becomes $(3-36 a)$

$$
\begin{aligned}
E I_{x} w_{1}^{\prime \prime \prime} & \left.+\left[M_{z_{0}} \phi_{1}\right]^{\prime \prime}-\tau E I_{x} \frac{\tau-1}{\tau}\left[v_{1}^{\prime \prime}-\phi_{0} w_{1}^{\prime \prime}-w_{0}^{\prime \prime} \phi_{1}\right) \phi_{0}\right]^{\prime \prime} \\
& +m \ddot{w}_{1}-s_{e} \phi_{1}-F_{z_{1}}=0
\end{aligned}
$$

$(3-36 b)$

$$
\begin{gathered}
\tau E I_{x}\left[v_{1}^{\prime \prime}-\frac{\tau-1}{\tau}\left(\phi_{0} w_{1}^{\prime \prime}+w_{0}^{\prime \prime} \phi_{1}+\underline{\phi_{0}^{2} v_{1}^{\prime \prime}}+\underline{2 \phi_{0} v_{0}^{\prime \prime} \phi_{1}}\right)\right]^{\prime \prime} \\
+m \ddot{v}_{1}-F_{x_{1}}=0
\end{gathered}
$$

$$
\begin{aligned}
G I_{d} \phi_{1}^{\prime \prime} & +\tau E_{x} \frac{\tau-1}{\tau}\left[\left(v_{1}^{\prime \prime}-\phi_{1} w_{1}^{\prime \prime}-w_{0}^{\prime \prime} \phi_{1}\right) w_{0}^{\prime \prime}+\underline{\left.2 v_{0}^{\prime \prime} \phi_{0} v_{1}^{\prime \prime}+v_{0}^{\prime \prime 2} \phi_{1}\right]}\right. \\
& -M_{z_{0}} w_{1}^{\prime \prime}+s_{e} \ddot{w}_{1}-J \phi_{1}+m_{y_{1}}=0
\end{aligned}
$$

The underlined terms are of higher order for $r>1$ and are discarded. When $T$ is sufficiently large that $\frac{T-1}{T} \approx 1$, equation (3-36b) becomes

$$
\tau E I_{x}\left[v_{1}^{\prime \prime}-\phi_{0} w_{1}^{\prime \prime}-w_{v}^{\prime \prime} \phi_{1}\right]^{\prime \prime}=F_{x_{1}}-m \ddot{v}_{1}
$$


The quantity $\beta$, deftined as

(3-38)

$$
\beta=v_{1}^{\prime \prime}-\phi_{0} w_{1}^{\prime \prime}-w_{0}^{\prime \prime} \phi_{1},
$$

is recognized as the time-dependent curvature, in the linearized

perturbation deflections, about the $n$ axis of the steady-state deformed

cross section. Thus it is expected that as $\tau+\infty$ the curvature $B$

must approach zero.

The dynamic linear moment $M_{z_{1}}$ is defined as

(3-39)

$$
M_{z_{1}} \equiv-\tau E I_{x} B
$$

Equations $(3-37)$ and $(3-36 a, c)$ can be restated, after use of (3-38)

and (3-39) together with $\tau \rightarrow \infty$, as

(3-40a) EI $x_{1}^{\prime \prime \prime \prime}+\left[M_{z_{0}} \phi_{1}\right]^{\prime \prime}+\left[M_{z_{1}} \phi_{0}\right]^{\prime \prime}+m \ddot{w}_{1}-s_{e} \phi_{1}-F_{z_{1}}=0$

(3-40b) $\quad \mathrm{M}_{z_{1}}^{\prime \prime}=\mathrm{F}_{\mathrm{x}_{1}}-m \ddot{v}_{1}$

(3-40c) GI $\phi_{d}^{\prime \prime}-M_{z_{0}} w_{1}^{\prime \prime}-M_{z_{1}} w_{0}^{\prime \prime}+s_{e} \ddot{w}_{1}-J \phi_{1}+m_{y_{1}}=0$

Since $\beta \rightarrow 0$, the acceleration term in the second equation could be expressed entirelv $\ln$ terms of $w$ and $\phi$ accelerations by working from integration of

(3-41) $\quad \ddot{v}_{1}^{\prime \prime}=\phi_{0} \ddot{w}_{1}^{\prime \prime}-w_{0}^{\prime \prime \phi}$

In this linearized unsteady system. $M_{z_{1}}(y, t)$ represents the moment at station $y$ due to the instantaraous chordwise inertial loads and applied 
Iinearlzed unsteady drag forces acting cutboard of the station. Because of the dependence of $M_{z_{1}}$ upon $w_{1}$ and $\phi_{1}$, equation (3-40b) cannot be uncoupled to allow for a separate calculation of $M_{z_{1}}^{\prime \prime}$. Thus, even though $v_{1}$ can be eliminated, (3-40) still involves three unknowns $w_{1}, \phi_{1}$, and $M_{2}$.

The purpose of this section has been to demonstrate analytically the connection between VCT representation of the cantilever wing, given by (3-24) and (3-25), and the VI system given in (2-1) and used in Ref. 1. It can be concluded that the VCT model remains valid for arbitrarily large bending stiffness ratio $\tau$, and that the drag coupling effect has been satisfactorally accounted for by the nonlinear elastic bending-torsion coupling terms. Since the forms obtained in (3-35) and (3-40) are but special cases of (3-24) and (3-25), actual solutions will be found, ustng the latter system only, for practical values of $\tau$. 


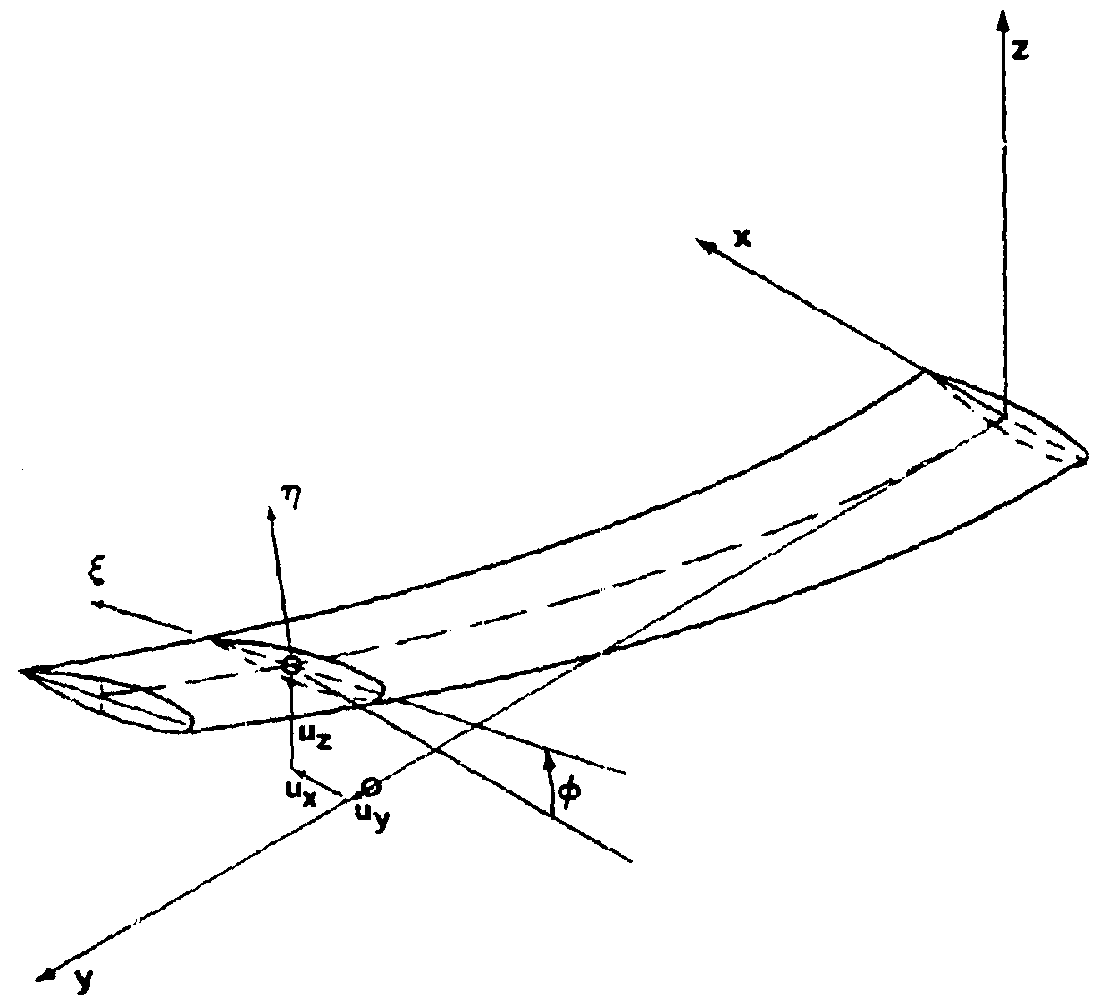

FIGURE 3-1 Coordinate System and Displacements

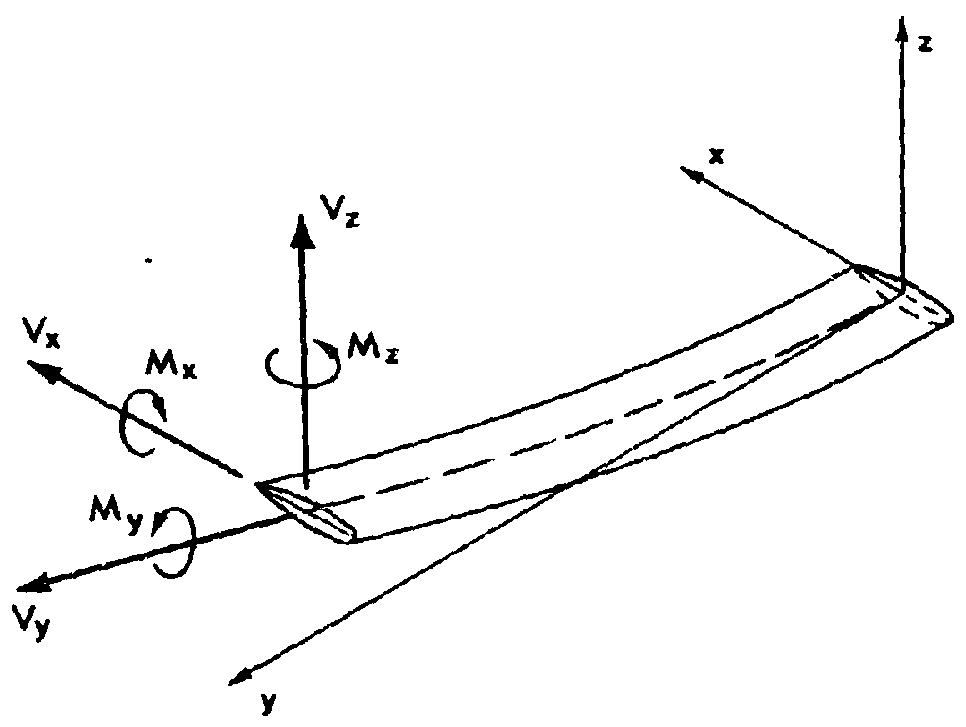

FIGURE 3-2 Stress Resultants and Moments 
Chapter IV

FLUTTER VELOCITY CONSIDERING VERTICAL BENDING/CHORDWISE BENDING/TORSION (VCT)

\section{A. Modal Equations for Smal1 Oscillatory Mot Ions About a Steady-State Deformation}

The model for toraton, transverse bending, and chordwise bending of the uniform cimtlever wing developed in the previous chapter is used to determine flutter velocity with of the same set of assumed modes used in $(2-1)$. Use of $n$ assuaed modes In torsicn。 $n$ in transverse bending, and $n$ in chordwise bending results in a wet of $3 n$ modal equations in terms of modal generallzed displacements. Since the assumed modes satisfy the natural as well as geometrical boundary conditions, which were obtained during the application of Hamflton's principle, Galerkin's method can be employed to transform the equations into algebraic relations in the generalized displacements. The nonlinear steady-state equilibriun equations (3-24) become nonlinear algebraic: equations in the steady-state generalized displacements, which are solved iteratively. These displacements determine the coefficients of the 11nearfzed unsteady model by applying Galerkin's method to (3-25). Then the velocity is determined for which simple harionic motion of this system is possible (neutral dynamic stability).

The steady aecodynamic loads for the equilibrtum equations (3-24) are specified in terms of incompressible strip theory. A typical alrfoll section (Fig. 4-1) has its zero-lift line inclined to the freestream velocity $\mathrm{V}$ by the angle $\alpha+\phi_{0}(y)$. The resultant steady lift $L_{a_{0}}$ (acting in a direction perpendicular to $V$ ) and the moment more given by 
$(4-1) \quad\left\{\begin{array}{l}L_{a_{0}}=2 \pi \rho v^{2} b\left(\alpha+\phi_{0}\right) \\ m_{a_{0}}=2 \pi \rho v^{2} b^{2}\left(l_{a}+a\right)\left(\alpha+\phi_{0}\right)\end{array}\right.$

A drag force $D$ is also present. These forces must be resolved into the axis system $x, y, z$ fixed with respect to the wing root. The required transformation is

$(4-2)\left\{\begin{array}{l}F_{z_{0}}=L_{a_{0}} \cos \alpha+D \sin \alpha \\ F_{x_{0}}=D \cos \alpha-L_{a_{0}} \sin \alpha\end{array}\right.$

Assuming $\sin \alpha \approx \alpha$ and $\cos \alpha=1$ and neglecting the $z$ coinponent of drag shows that the steady aerodynamic forces to be used with (3-23) are

$(4-3)\left\{\begin{array}{l}F_{z_{0}}=2 \pi \rho v^{2} b\left(\alpha+\phi_{0}\right) \\ F_{x_{0}}=2 \pi \rho v^{2} b\left(c-\alpha^{2}-\alpha \phi_{0}\right) \\ m_{y_{0}}=2 \pi \rho v^{2} b^{2} \lambda\left(\alpha+\phi_{0}\right)\end{array}\right.$

The drag force represented by $\mathrm{C}$ is constant spanwise, and the definitions in (2-22) have been adapted.

The steady-stace deflections are now expressed in terms of the assumed modes (cf. Appendix A for definitions) 
$(4-4) \quad\left\{\begin{array}{l}w_{0}=\sum_{i=1}^{n} f_{w_{i}} q_{w_{i}}^{0} \\ v_{0}=\sum_{i=1}^{n} f_{v_{i}} q_{v_{i}}^{0} \\ \phi_{0}=\sum_{i=1}^{n} f_{\phi_{i}} \cdot q_{\phi_{i}}^{0}\end{array}\right.$

Bending mole shape functions $f_{w_{1}}$ and $f_{v_{1}}$ are identical. Substituting (4-4) into (3-24) and (4-3) and applying Galerkin's procedure leass co

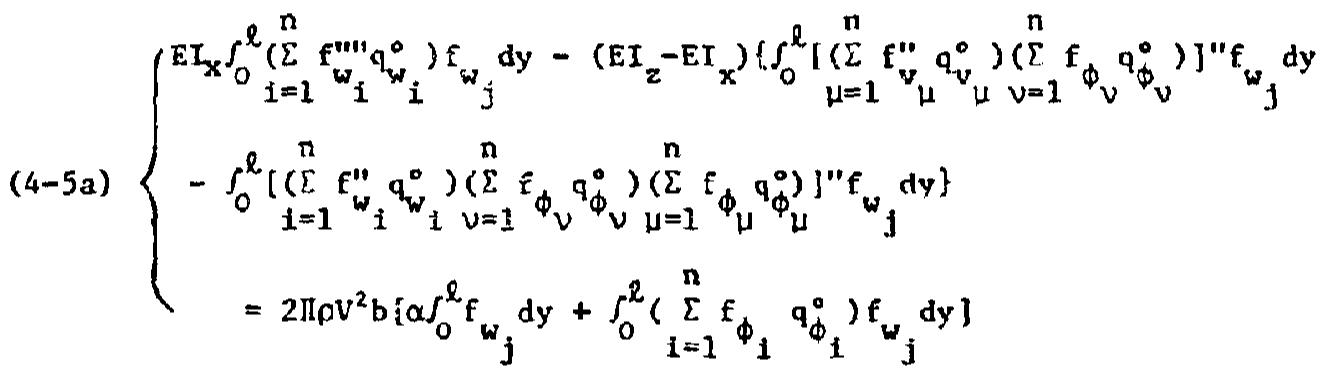

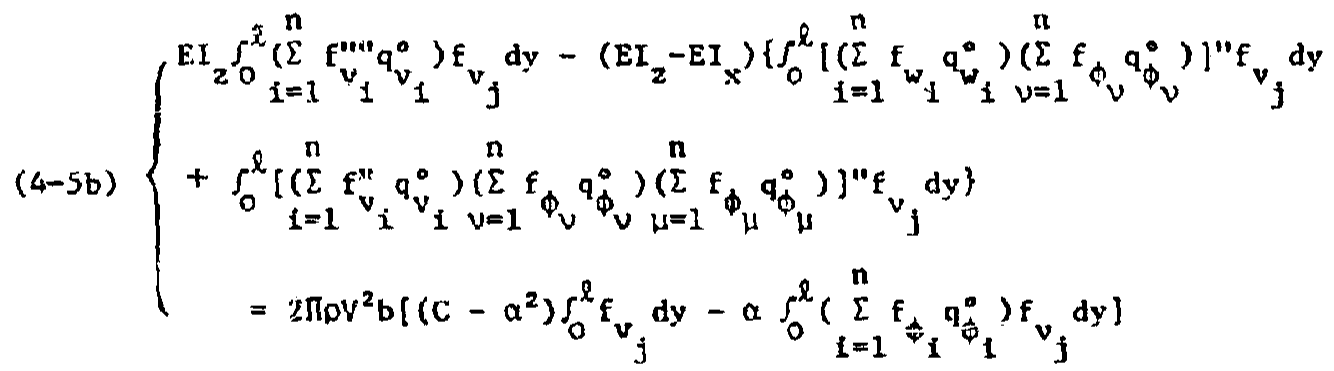

$$
\begin{aligned}
& (1 \leq j \leq n)
\end{aligned}
$$

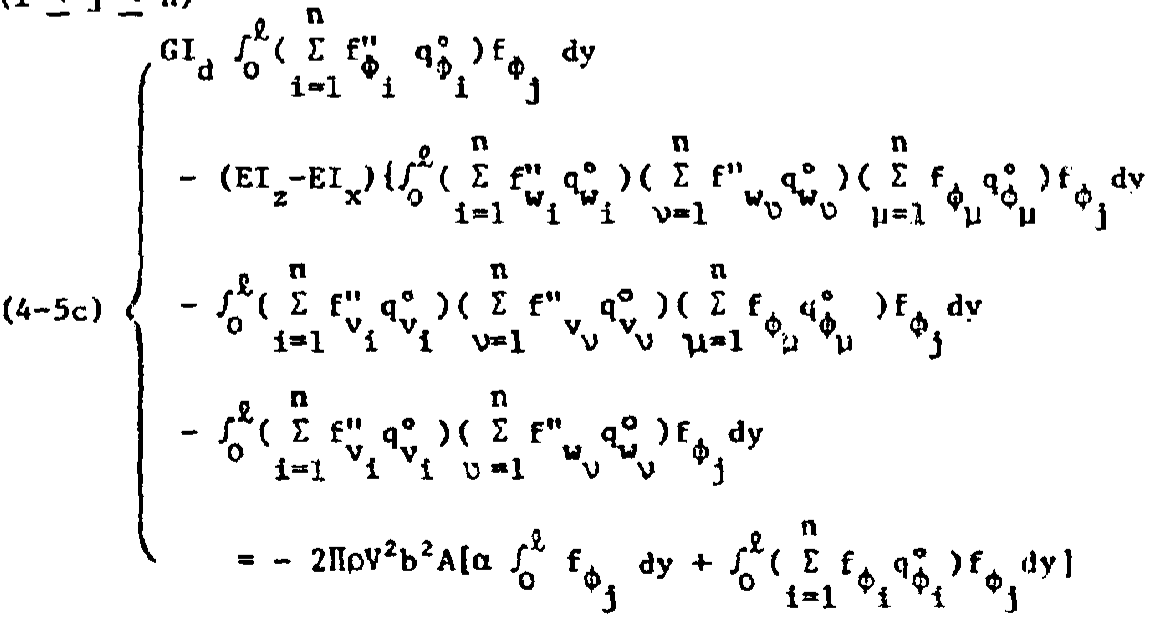


The indicated differentiations in the first two equations are carried out, and appropriate integrations by parts of the resulting modal integrais is performed. The first terms of each equation are then expressed in terms of modal natural frequencies as in (2-10) and (2-11) which leads to the form

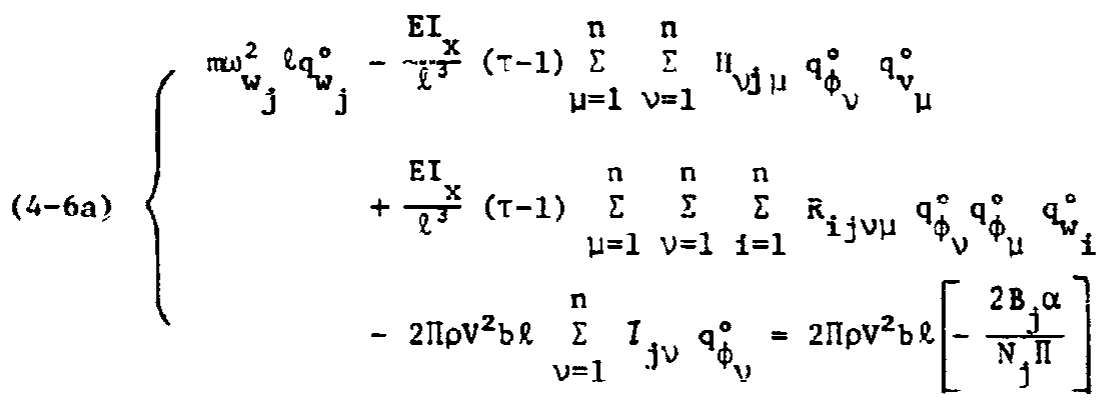

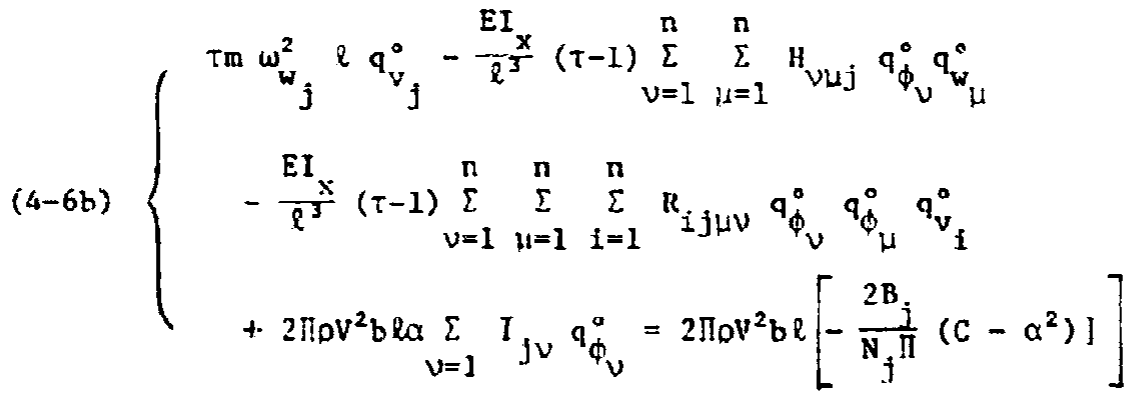

$(1 \leq j \leq n)$

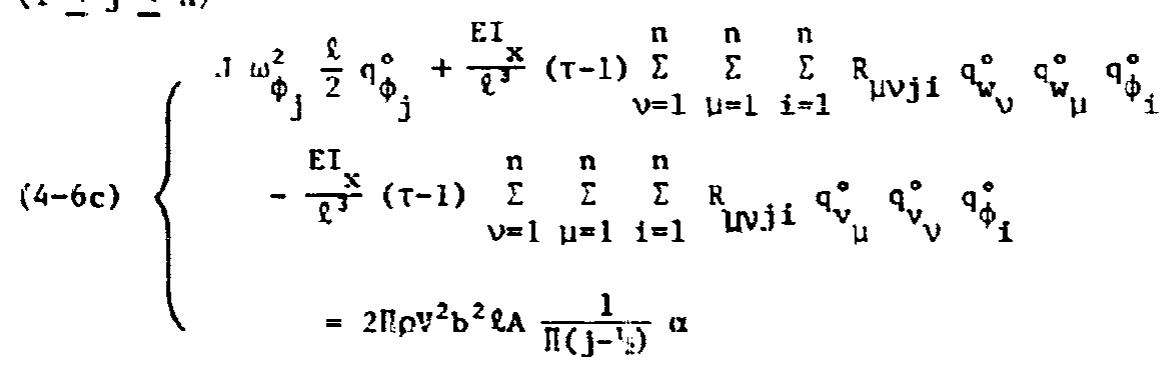

$B_{f}$ and $N_{f}$ are properties of the assumed bending todes described in Appendix $A$, and $I_{1 j}$ represent the modal integrals previously encountered and defined in (2-13). The nonl inear bending-torsion coupling terms give rise to two new forms of modal integrals, 
(4-7) $\quad H_{j \mu \nu}=\int_{0}^{1} E_{\phi_{j}}(\tilde{y}) f_{w_{\mu}}^{\prime \prime}(\tilde{y}) f_{w_{\nu}}^{\prime \prime}(\tilde{y}) d \tilde{y}$

(4-8) $\quad R_{i j \mu \nu}=\int_{0}^{1} f_{w_{i}}^{\prime \prime}(\tilde{y}) f_{w_{j}}^{\prime \prime}(\tilde{y}) f_{\phi_{\mu}}(\tilde{y}) f_{\phi_{\nu}}(\tilde{y}) d \tilde{y}$

Equations (4-6) can be arranged in the final format used for computations by dividing the torstion equation by $\Pi \rho V^{2} l b^{2}$ and the two bending equations by $\Pi p V^{2} l b$, using modal natural frequency relations in $(2-14)$ and (2-15), and nondimensionalizing with the parameters given in (2-16) and $(2-26)$.

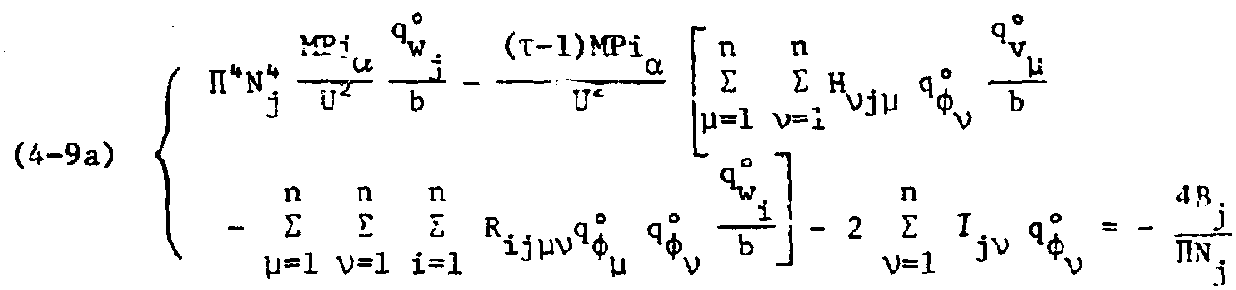

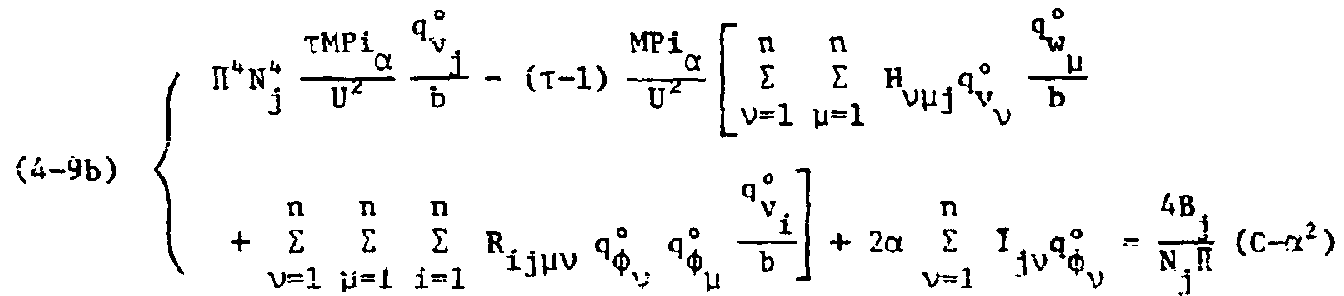

$(\mathbf{i} \leq \mathbf{j} \leq \mathbf{n})$

$$
\begin{aligned}
& \left(\left[\frac{1}{2} \Pi^{2}\left(j-\frac{1}{2}\right)^{2} \frac{M i_{\alpha}}{U^{2}}-A\right] q_{\phi_{j}}^{0}\right. \\
& (4-9 c)\left\{+(\tau-1) \frac{M P i}{U^{2}}\left[\sum_{v=1}^{n} \sum_{\mu=1}^{n} \sum_{i=1}^{n} R_{\mu \nu j i} \frac{q_{v}^{0}}{b} \frac{q_{w_{\mu}}^{\circ}}{b} q_{i}^{0}\right.\right. \\
& \left.-\sum_{v=1}^{n} \sum_{\mu=1}^{n} \sum_{i=1}^{n} R_{\mu v i j} \frac{q_{v}^{0}}{b} \frac{q_{v}^{0}}{b} q_{\phi_{i}}^{0}-\sum_{\nu=1}^{n} \sum_{v=1}^{n} H_{j \cup L} \frac{q_{v}^{0}}{b} \frac{q_{i \mu}^{0}}{b}\right] \\
& =2 A a \frac{1}{\pi\left(j-k_{2}\right)}
\end{aligned}
$$


A solution to (4-9) can be found for a given wing configuration once drag $C$, root angie of attack $\alpha$, and dimensionless speed $U$ have been specified. The solution procedure involves Newton's method and is described in Appentix $C$.

When simple hatmonic motion is assumed, the linestized parcurbation eịuations of motion (3-25) are converted into linear alpebraic equations via the same steps described in Chapter II In deriving modal equations (2-18) from (2-1). The percurbation displacements are expanded in rerms of $n$ assumed modes and generalized displacements,

$(4-10)$

$$
w_{l}(y, t)=\sum_{i=1}^{n} f_{w_{i}}(v) q_{w_{i}} e^{i w t}
$$

$$
v_{1}(y, t)=\sum_{l=1}^{n} f_{w_{i}}(y) q_{v_{l}} e^{i \omega s t}
$$

$$
\phi_{1}(y, t)=\sum_{1=1}^{n} i_{\theta_{t}}(y) \phi_{\theta_{1}} e^{i \omega t}
$$

where $q_{u_{1}}$ and $q_{v_{1}}$ have dimensions of lenxth, wereas $q_{\theta_{1}}$ are dimenstonless. Anteclpating solution for fluter boundaries using the V-g method, structural dampins. \&. Is included by Introducing a coeplex elastic adulus, and the stmple harmonte afrloads are assured to have been expressed is cerm of unlt generalized forces as in (2-9). With the addition of the fore-and-aft bending degree of freedom. the generilized forces now appanr as

$(4-3,1 a)$ $(1 \leq 5 \leq n)$

$$
\int_{0}^{i} f_{2}\left(s_{1} \cdot w_{1} ; t\right) t_{n} d y
$$

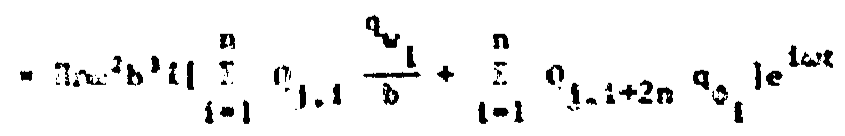


(4-11b) $\quad \int_{0}^{2} F_{x_{1}}\left(\theta_{1} \cdot w_{1}: t\right) f_{v_{j}} d y$

$$
=\pi D w^{2} b^{3} R \mid \sum_{i=1}^{n} Q_{j+n, 1} \frac{Q_{1}}{b}+\sum_{i=1}^{n} Q_{i+n, 1+2 n} q_{i} 1 e^{1 w t}
$$

$(1 \leq 1 \leq \pi)$

$$
\begin{aligned}
& (4-11 c) \quad \varepsilon^{i} m_{v}\left(s_{1} \cdot v_{1}: t\right) f_{s} d v
\end{aligned}
$$

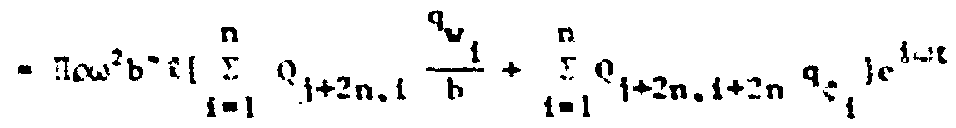

The owission of colums in the arrav o, t, havlag $n+1 \leq 1=2 n$ refleces the assumetion that there is no dependence of unsteadv alrloads upon forcmand-aft wotions $v_{1}$.

Aiter Galerkin's bethod has been i=plewented for equations (3-25) by the same manipulations required to develop $(2-17)$. the svsten of In godal equations which deteraine linear stabllits thout the stowt equilibrlus deflectims appoar in the fors

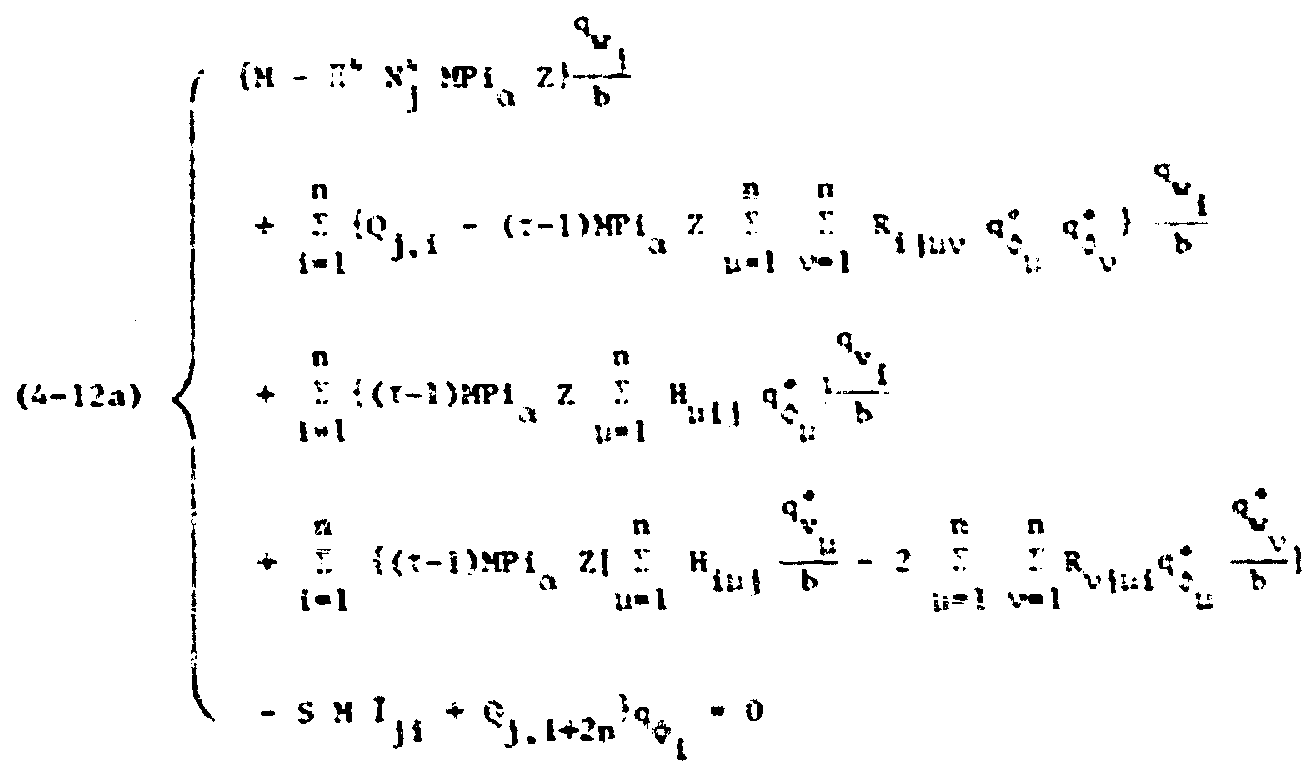




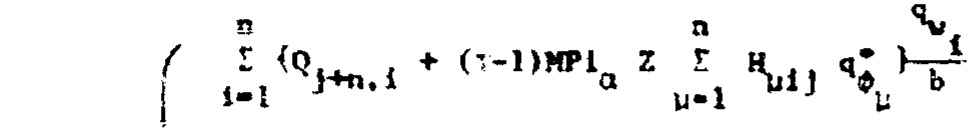

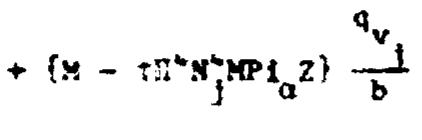

$$
\begin{aligned}
& (i-12 b)
\end{aligned}
$$

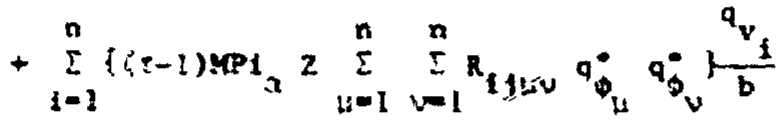

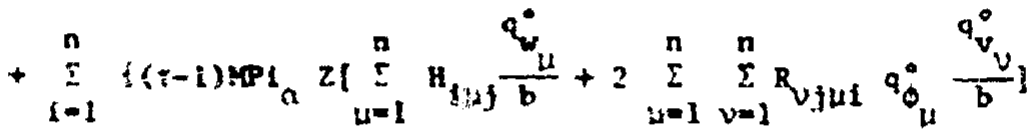

$$
\begin{aligned}
& +Q_{1+n .1+2 n^{i q}}=0
\end{aligned}
$$

$(2 \leq 1 \leq n)$

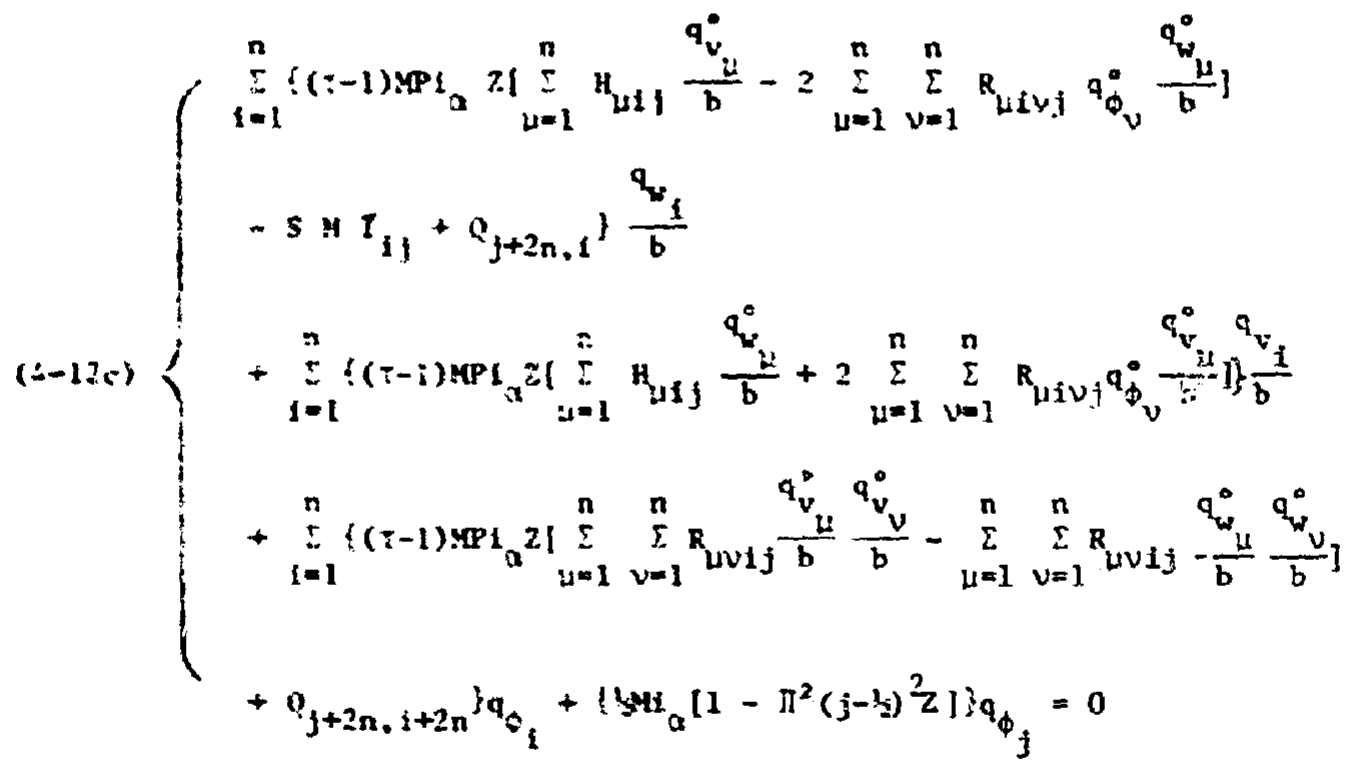

Here "Huv and $R_{1 j a v}$ are the mandal integrals defined in $(4-7)$ and (4-3), which appear in this system multiplled by steady-state generated displacemes. The cofficients of terms which couple the szeady deflections into this system must first be constructed by the indicatyd sumations bcfere the eigenvalues can be deterained. 
The unit generalized forces required in (4-12) will now be expressed in terms of the two-dimensional incompressible unsteady aerodynamics adopted for (2-2) and (2-3). It will be nacessary, as it was for the steady aerodynanics, to transfer the airloads from wind orlented axes to the coordinate system fixed at the wing root. For the present case, the circulatory part of the unsteady lift will be assumed perpendicular to the direction of the freestream velocity, whereas the noncirculatory portion will be assumed to act normal to the chord of the airfoil section in its steady-state deflected position. The inclusion of unsteady leading-edge suction effects will be considered in the next chapter.

To separate the circulatory and noncirculatory contributions to the lift, $L_{a}$ in $(2-2)$ and $(2-3)$ can be rewritten in the forr. (cf. Eq. (4-126) of Ref. 5)

$$
\begin{aligned}
\mathrm{La}\left(w_{1}, \phi_{1} ; t\right)= & \Pi \rho b^{3} \omega^{2}\left[\frac{\widetilde{w}_{1}}{b}+\frac{i \widetilde{\phi}_{1}}{k}+a \widetilde{\phi}_{1}\right] e^{i \omega t} \\
& +\Pi \rho b^{3} \omega^{2} C(k)\left[-\frac{2 i}{k} \frac{\tilde{w}_{1}}{b}+\frac{2 \tilde{\phi}_{1}}{k^{2}}+\frac{2 i}{k}\left(s_{2}-a\right) \tilde{\phi}_{1}\right] e^{i \omega t}
\end{aligned}
$$

Here the first term on the right represents noncirculatory lift $\mathrm{L}_{\text {NC. }}$ and the second term the circulatory portion $\mathrm{L}_{a_{C}}$. Referring to Fig. 4-2, these terms can be expressed as resultant forces in the $x z$ Erame by

$\left\{\begin{array}{l}\mathrm{F}_{\mathrm{z}_{1}}=\mathrm{L}_{\mathrm{a}_{\mathrm{C}}} \cos \alpha+\mathrm{L}_{\mathrm{a}_{\mathrm{NC}}} \cos \phi_{\mathrm{O}} \\ \mathrm{F}_{\mathrm{x}_{1}}=-\mathrm{L}_{\mathrm{a}_{\mathrm{C}}} \sin \alpha+\mathrm{L}_{\mathrm{a}_{\mathrm{NC}}} \sin \phi_{\mathrm{O}}\end{array}\right.$ 
Kaking the assumptions $\cos \alpha=1$ and $\cos \phi_{0}=1$ yields the 1dantity (4-15) $\quad F_{z_{1}}=\mathrm{L}_{\mathrm{a}}\left(w_{1}, \Phi_{1} ; t\right)$

When it is assumed that $\sin \alpha \approx \alpha$ and $\sin \phi_{0} \approx \phi_{0}$, the chordwise force components become

$$
\begin{aligned}
(4-16) \quad F_{x_{1}}= & \pi \rho b^{3} \omega^{2} \alpha c(k)\left[\frac{2 i}{k} \frac{\tilde{w}_{1}}{b}-\frac{2 \Phi_{1}}{k^{2}}-\frac{2 i}{k}\left({ }^{1} / 2-a\right) \tilde{\Phi}_{1}\right] e^{i \omega t} \\
& +\pi \rho b^{3} \omega^{2} \phi_{c}\left[\frac{\tilde{w}_{1}}{b}+\frac{1 \tilde{\Phi}_{1}}{k}+a \Phi_{1}\right] e^{i \omega t}
\end{aligned}
$$

Preparatory to sreps that lie ahead, it can be seen that when the unit generalized forcss are determined from (4-11) with the modal series for $\phi_{0}, w_{1}$, and $\Phi_{1}$ inserted, modal integrals of the forms

$$
\begin{aligned}
& \int_{0}^{l} f_{v_{1}}(\tilde{y}) f_{v_{j}}(\tilde{y}) f_{\phi_{v}}(\bar{y}) d \bar{y} \\
& \int_{0}^{1} f_{v_{i}}(\tilde{y}) f_{\phi_{j}}(\tilde{y}) f_{\phi_{v}}(\bar{y}) d \bar{y}
\end{aligned}
$$

will be encountered as a consequence of the noncirculatory contribution to (4-16). Since these integrals do not occur elsewhere and the effect they introduce is expected to be minor, the noncirculatory contribution to $\mathrm{r}_{\mathrm{x}_{1}}$ is neglected altogether. This simplification has the result that the unsteady noncirculatory force illustrated in Fig. 4-2 acts in the $z$ direction at all spanwise stations.

The remainiag circulatory contribution to $F_{x_{1}}$ can be expressed, using the notation in (2-3) and after some manipulation, as 
(4-17) $\quad F_{x_{d}}=\pi \rho b^{3} \omega^{2} \alpha l\left(1-L_{w}\right) \frac{\tilde{w}_{1}}{b}+\left[L_{\phi}-\frac{1}{2}+\frac{1}{k}+A\left(1-L_{w}\right)\right] \tilde{\phi}_{1} e^{f \omega t}$

With the aerodynamic loads given in $(4-17),(4-15)$, and $(2-2)$, where $m_{y_{1}}\left(\phi_{1}, w_{1} ; t\right)=m_{a}\left(w_{1}, \phi_{1} ; t\right)$. the unti generalized forces can be $c$;veloped, starting from (4-11), by means of the same steps followed in assembling $(2-22)$. The final result is

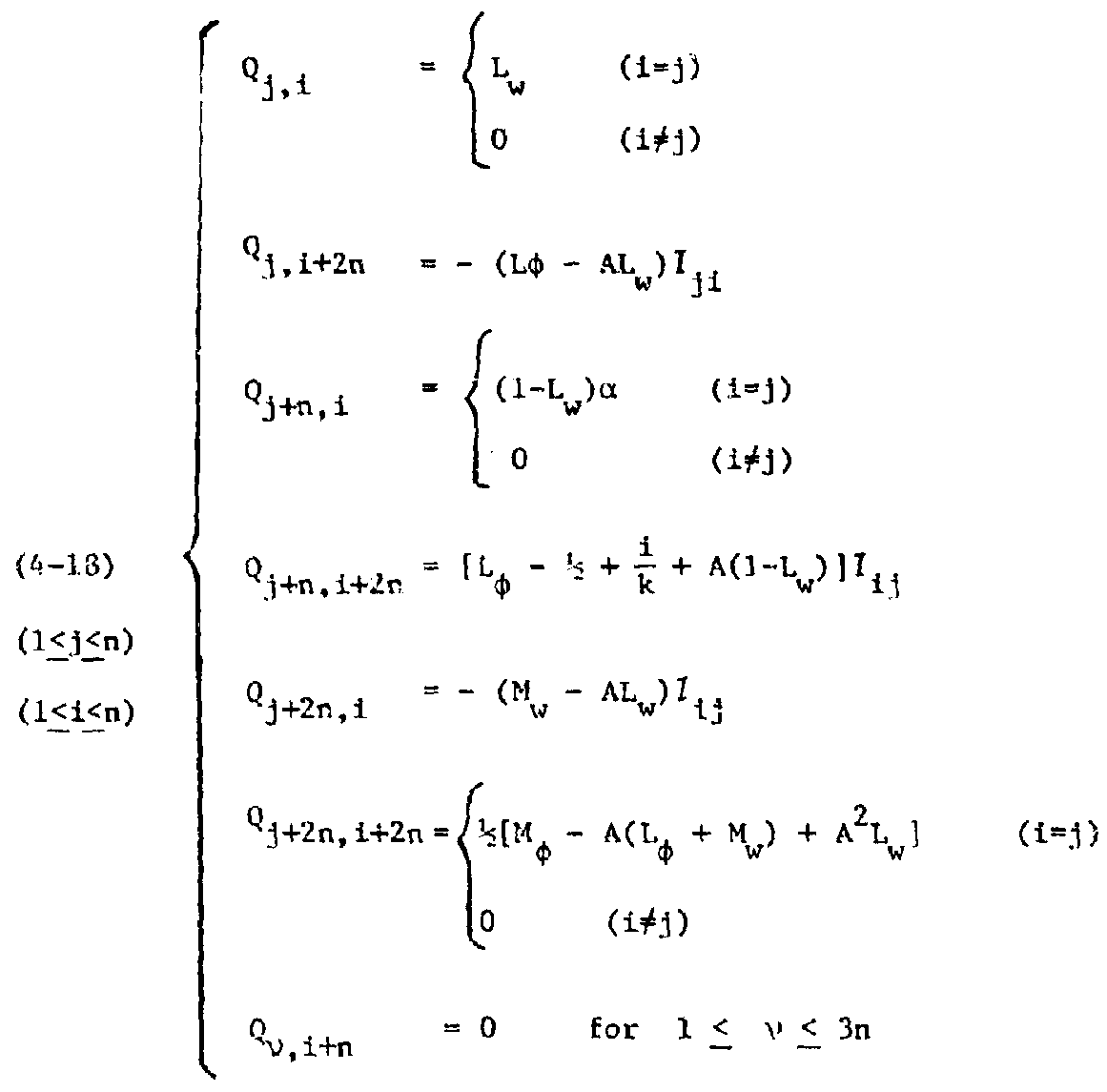




\section{B. Solution Procedure for Flutter Velocity of a Lifting Wing}

The in modal equations (4-12), with (4-18) inserted, can be expressed in matrix form

$(4-19) \quad\left(\left[M_{s}\right]+[Q]\right)\{q\}-z\left[k_{s}\right]\{q\}=0$

The mass and stiffness matrices $M_{s}$ and $K_{s}$ are real and symmetric and are written out in Fig. 4-3. The terms in $\mathrm{K}_{\mathrm{s}}$ include sums of products of modal integrals and the steady equilibrium generalized displacements found as solutions of (4-9). The aerodynamic matrix Q whose elements appear in (4-18), is complex and nonsymmetric. Equation (4-19), therefore, represents a complex eigenvalue problem for the complex frequency parameter $Z$. Its solution yields damping. speed, and frequency as in the case of (2-25) and (2-27).

The logic used to compute neutrai stability conditions from (4-19) is diagrammed in Fig. 4-4. The primary difficulty encountered when steady deflections are introduced is that a preliminary estimate of speed $U_{e}$ must be made before tine eigenvalue problem can be solved. Steady deflections for $U_{e}$ are used to generate coefficients in $K_{s}$, and unsteady aerodynamic loads are then computed for large enough reduced frequency that the eigenvalue corresponding to the aeroelastic mode which flutters has negative (stable) damping $g$. Successively smaller values of $k$ are then substituted and aerodynamic terms recomputed. The eigenvalues are recalculated until positive damping occurs for the flutter mode. From this point a zero-locating subroutine determines the reduced frequency for which damping $g$ is zero, whereupon one can calculate flutter speed $U_{F}$. The entire procedure 
is repeated with a new estimate $U_{e}$ until the speeds $U_{e}$ and $U_{F}$ are adequately matched. The flutter speed determined for zero lift serves as a good first estimate for $\mathrm{v}_{e}$. With care, close agreement of the two speeds can be achleved in three or four iterations.

Three assumed modes in each degree of freedoin, corresponding to a system order of 9 , are found to give adequate convergence for all cases. The model integrals were computed to allow $n \leq 4$, a task which required nuserical integration of 100 quantities of rype $R_{i j h \nu}$ and 40 of type $\mathrm{H}_{\mathrm{i} \mu \mathrm{V}}$.

\section{The Nonlinear Elastic Coupling iernis}

The need to retain ail Eerms in (3-22), including third degree nonlinear, in order to model adequately the nonlinear elastic bendingtorsion coupling mechanism is now demonstrated by means of typical applications. Of course, neglecting higher-order nonlinear effects would have the appeal of reducing complexity. For example, removal of all third-degree nonlinear cerms would result in the elimination of all terms containing quadruple modol integrals $R_{i j u \mu}$ from both the nonlinear steady equilibrium system and the linearized dynamic stability analysis. In order to examine the effects of such approximations, numerical experiments were conducted wherein higher degree terms in both the steady and unsteady modal equations were neglected. First, static deflections are considered. Clsarly, if the equations do not adequately represent the steaay-state deflections of the wing over a realistic range of lifting conditions, then any lineartzed unsteady perturbation analysis based on the same level of 
approximation cannot be expected to succeed. The nonlinear steady

equations (3-23) frili be solved at three levels of approximation:

(1) Linear terms only retained.

(2) Second degree monlinear terms included.

(3) All nonlinear terms included.

The linear set of modal equations is just $(4-9)$ with the nonilinear

cerms removed:

$(4-20)$

$$
\begin{aligned}
& \Pi^{4} N_{j}^{4} \frac{M i_{Q}}{U^{2}} \frac{q_{w}^{0}}{b}-2 \sum_{v=1}^{n} I_{j v} q_{\phi_{v}}^{0}=-\frac{4 B_{j}}{\pi N_{j}} \\
& \Pi^{4} \mathrm{~N}_{j}^{4} \tau \frac{\mathrm{MPi}_{\alpha}}{\mathrm{U}^{2}} \frac{\mathrm{q}_{\mathrm{v}}^{0}}{\mathrm{~b}}+2 \alpha \sum_{\nu=1}^{n} I_{j \nu} q_{\phi_{v}}^{0}=-\frac{{ }^{4 B} j}{\Pi_{j}}\left(c-\alpha^{2}\right) \\
& {\left[\frac{1}{2} \Pi^{2}\left(j-1_{2}\right)^{2} \frac{M i}{U^{2}}-A\right] q_{\phi_{j}}^{j}=\frac{2 A \alpha}{\Pi\left(j-\frac{1}{2}\right)}}
\end{aligned}
$$

The torsion equations are now seen to be $n$ uncoupled and immediatelysolveable relations for the $n$ generalized displacements $\vartheta_{\phi_{j}}^{0}$. Tine results permit solution for the bending displacements, thus eliminating the need for matrix operations. Since all elastic coupling terms are absent, deflections $w_{0}$ and $\phi_{0}$ are independent of the stiffness ratio $\tau$ and the steady drag parameter C . A very important consequence is that the mechanism by which drag influences divergence is missing. One concludes that $(4-20)$ is a particularly poor representation for steady deflections in the presence of steady drag.

Retention of the second-degree nonlinear effects from (4-9) involves adding to (4-20) all terms containing the tiple modal integrals $\mathrm{H}_{\text {ivM }}$ - The system becomes fully coupled, and solutions are found by 
the saste iterative scheme described in Appendix c for solving the full nonlinear system.

The behavior of the second degree nonlinear solution is compared with the linear deflection from $(4-20)$ in Figs. 4-5 and 4-6. Here it is shown how the deflection in the first vertical bending mode and the first torsion mode vary with increasing speed for a fixed angle of attack $\alpha=0.01$ radions of the wing root. The paraneters $M, \bar{F}$, $I_{\alpha}$, A, and $T$ for these examples correspond to the idealization of a sailplane wing discussed in Chapter VI, and sufficient modal convergence is assured by losing $n=3$. Numbers on the ordinate of Fig. 4-5 show the actual twist in radians of the wingtip due to the first torsion mode; the vertical deflection in semichords of the wingtip due to $q_{w_{1}}^{0}$ is just twice the value read from the ordinate of Fig. 4-6.

The important influence of drag on the steady deflectiens is evident. But there is pour correspondence between the second degree nonlinear deflections and the divergence speeds indicated in Fig. 4-5, which are solutions of the linear static stablity determinant (2-28). This discrepancy is most pronounced when $C=0$, and the rapid divergence of the second-degree nonlinear deflections for all values of drag suggests trouble with the second-degree approximation.

Figures 4-7 through 4-10 show solutions of the complete nonInear system (4-9) for the same wing coiffiguration, together with the linear results. Correlarion with the divergence predictions appears to be excellent, and the sudden blowing up of deflections characteristic of the second-degree noninear solutions is not encountered. Since the iterative procedure used to solve for aonlinear deflections commences 
with the linear solution as an initial estimate, nonlinear solutions which do exist for $\mathrm{C}=0$ above the classical divergence speed cannot be obtained.

The full nonlinear solutions conform with nonlinear behavior expected by intuition. For zero drag, the vertical bending and torsion deflections should fall below the linear solution owing to the effective increase in stiffness "seen" by each degree of freedom due to deformation in the other. This effect is observed. The slight rearward chordwise displacement (Fig. 4-9) for $\mathrm{C}=0$ when $w_{0}$ and $\phi_{0}$ are large comes from bending-torsion coupling, and the negative contribution of the second torsion rode (Fig. 4-?U) reflects a redistribution of elastic twist toward the wingtip where the curvature due to $w_{0} 1 s$ less. Drag alters the deformed state radically and causes large displacements at much lower speeds.

A clue to the reason why the second-degree terms alone are inadequate is found by looking at the sensitivity of solutions to changes in the bending stiffness ratio $\tau$. The second degree nonlinear solution for bending deflections, given in Fig. 4-11, reveals increasingly poor behavior as $\tau$ is increased. Conversely, the complete nonlinear solution behaves as intuition would anticipate, becoming insensitive to changing $\tau$ as this parameter grows toward $\infty$ (Fig. 4-12).

In Chapter III the behavior for $\tau \rightarrow \infty$ of the steady nonlinear equilibrium equations was analytically investigated. In the discussion following equation (3-33) it was pointed out that the quantity $v_{0}^{\prime \prime}-\phi_{0} w_{0}^{\prime \prime}$ represents the actual curvature about the principal axis of chorduiss bending for the deflected afrfoil section, and that this 
quantity should vanish as $\tau+\infty$. In this limit any chordulse deflection is a geometric consequence af elastic coupling between $\phi_{0}$ and $w_{0}$; terms which contain the product $\tau\left(v_{0}^{\prime \prime}-\phi_{0} y_{0}^{\prime \prime}\right)$ approach a finite value. Now, referring to equations (3-24), it can be seen that in the second degree approximation this product is retained in the second equation but not in the vertical bending equation (where $w_{0}^{1} \varphi_{0}^{2}$ is dropped but $v_{0}^{\prime \prime} \phi_{0}$ retained). Likewise in the torsion equation, $w_{0}^{\prime \prime 2} \phi_{0}$ is dropped but $v_{0}^{\prime \prime} w_{0}^{\prime \prime}$ is kept. The result is that terms containing ' $\left[v_{0}^{\prime \prime}\right.$ remain and blow up in the limit $T \rightarrow \infty$. Accordingly, the conclusion is reached that, for structures representative of aircraft: wings for which $\tau$ is reasonatily large, the third-degree terms containing the product $w_{0}^{\prime \prime} \Phi_{o}$ must be ratained. It may be added that, aithough this elastic coupling effect was neglected in the helicopter blade equations developed in Ref. 7 , in that case the ordering scheme required $\tau$ to be on the order of unity. Another point is that the remaining third-degree terms in (3-24), which contain the product $v_{0}^{\prime \prime} \phi_{0}$, actually can be neglected when $\tau$ is large.

Since it has been determined that third degree terms cannot be excluded from the steady nonlinear equilibrium analysis, it foliows that they must also be kept in the linearized unsteady porturbation equations (3-25). The orlginal dynamic stability equations used early in this investigation, on the other hand, consisted of a lineartzed unsteady perturbation system based on the second-degree nonlinear approximation. That is, they are the result of removing all terms containing the modal integrals $R_{i j \mu v}$ from (4-12). Irregular behrvior of the flutter speed with increasing angle of attack $\alpha$ was discovered, 
as was an extreme sensitivity to large $T$ which could not be justifled physfially. Furthermore, linear steady-state deflections were used-a particularly unsuitable approximation for inclusion of steady drag. Examples of these original flutter calculations appear in Figs. 4-13 and 4-14. The former gives a compapisen of the early results with the full analysis by (4-12) for the sailpient example, showing how the stmpler approximation differs signiflcarisy in flutter speed even in the presence of moderate steady deflections. Figure 4-I4, based on a different wing configuration, gives an idea of the difficulty encountered for large $\tau$ when using the simpler analysis. (Note that the steady deflections are quite small in view of the fact that this example is a large-aspect-ratio wing). All of these stability buundaries abruptly terminate, at which point the eigenvalue solutions began to behave erratically. In contrast, flutter solutions of (4-19) can be obtained for arbitrarily large sceády deformations-- indeed for tip deflections well beyond practical Iimits of material IInearity.

\section{Comparison of VT and VCT Calculations}

The flutter velocities found using the VCT model can be shecked directly against velocity computed fiom the VT model developed in Chapter II. Although such a comparison restricts the former method to the special notilifting case $w_{0}=\phi_{0}=0$, its basic approach of linearized perturbation motions about a steady-state deflection solution can nevertheless be tested by inclusion of steady drag. This is because the drag effect enters the stability determinant through coefficients depending on $v_{0}$. The chordwise bending equation of 
motion is uncoupled when $w_{0}=v_{y}=0$. and fluter sodes involve Just vertical bending and sorsion.

The VCT stabllity equations were analyclcally reciuced to the IT form (2-1) in the last chapeer by specifying $a-0$ and $t+\infty$. This agreement is reflected in numerical results, as evidenced by Fig. $4-15$. For an exceptionally large magnttude of drag. $c=0.04$. chosen to magnify the iqportance of drag coupling and hence the steady deflections in the stability analysis, the two methods are used to compute flutter spiteds for the same nonlifting configuration. The FCT analyses were mede for $\tau$ zanging from 1 to 10,000 .

As is always true for $E_{z}=E_{x}$, the drag coupling effect vanighes at $T=1$ with flutter speed unaffected by drag. At the other extreme, for $\tau=10,000$ the computed flutter speeds for $c=0.04$ differ by a mere 0.052\%. The Ref. 1 flutter speeds calculated for this same configuration are also shown and agree quite well.

When one examines the VCT dependence of flutter speed on $\tau$ (Fig. 4-15), the effect of drag is apparently insensitive to $T$ over the range of this parameter representative of practical aircraft applications. Hence the VT system (2-1) does not suffer by Its inherent assumption that $\tau \rightarrow \infty$.

Table 4.1 presents a further comparison of results from the two approaches for a different configutation, in which modai convergence is emphasized. Of course, since $\tau=50$ exact agreement cannot be expected.

Convergence of flutter speeds and frequencies and also mode shapes for the VCT solutions is dacumented in Table 4.2 for zero steady 
1ift and In Table s.J for a 11 feing condition speclfled by a - .01

rad. Iri the latzer case, tha apparent slower coavernence is relased

co the steady lifting deflections (also cabulated). Vhleh also yagr ytch $n$. 


\begin{tabular}{|c|c|c|}
\hline$n$ & $U_{F} \cdot \mathrm{V}$ & $U_{\mathrm{F}}$ VCT \\
\hline 2 & 4.260423 & 4.258457 \\
3 & 4.260479 & 4.258351 \\
4 & 4.260882 & 4.258335 \\
5 & 4.260889 & 4.258336 \\
\hline
\end{tabular}

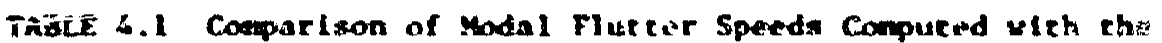
IT and VCT Sxiters. (M-40..P-0.4. $q_{a}-0.25$. A $0.1 .5=0.1 .7=50 . \mathrm{C}=0.08$ )

\begin{tabular}{|c|c|c|c|c|c|c|c|c|}
\hline & \multicolumn{2}{|c|}{$\begin{aligned} n & =1 \\
u_{F} & =4.15027 \\
\Omega_{F} & =0.85254\end{aligned}$} & \multicolumn{2}{|c|}{$\begin{aligned} n & =2 \\
U_{F} & =4.183899 \\
\Omega_{F} & =0.088768\end{aligned}$} & \multicolumn{2}{|c|}{$\begin{aligned} n & =3 \\
u_{F} & =4.183883 \\
\Omega_{p} & =0.88757\end{aligned}$} & \multicolumn{2}{|c|}{$\begin{aligned} n & =\dot{s} \\
U_{F} & =\dot{s} 183916 \\
\Omega_{F} & =0.88758\end{aligned}$} \\
\hline & AMPL. & PHASE & AMPL. & PHASE & AMPL. & PHASE: & AMPL. & PHASE \\
\hline & 2.1800 & $224^{\circ} .54^{\circ}$ & 2.0650 & $223.87^{\circ}$ & 2.0659 & $223.87^{\circ}$ & 2.0659 & $223.87^{\circ}$ \\
\hline & - & & 0.6345 & $-46.85^{\circ}$ & 0.6309 & $-46.84^{\circ}$ & 0.6308 & $-46.85^{\circ}$ \\
\hline & - & & - & & 0.0107 & $-4.46^{\circ}$ & 0.0107 & $-4.26^{\circ}$ \\
\hline & - & & - & & - & & 0.0022 & $1.89^{\circ}$ \\
\hline$q$ & 1.0 & $0^{\circ}$ & 1.0 & $0^{\circ}$ & 1.0 & $0^{\circ}$ & 1.0 & $0^{\circ}$ \\
\hline & - & & 0.03067 & $19^{\circ} .53^{\circ}$ & 0.03075 & $199.70^{\circ}$ & 0.03075 & $199.70^{\circ}$ \\
\hline$q_{\phi}$ & - & & - & & 0.00340 & $-5.55^{\circ}$ & 0.00339 & $-5.67^{\circ}$ \\
\hline$q_{\phi}$. & - & & - & & - & & 0.0059 & $220.49^{\circ}$ \\
\hline & & & & & & & & \\
\hline
\end{tabular}

TABLE 4.2 Modal Convergence for Flutter at Zero Steady lift. $(M=9.4$, $\mathrm{P}=0.01, i_{\alpha}=0.25, \mathrm{~A}=0.1, \mathrm{~S}=0.1, \tau=25 ., \mathrm{C}=0$ ) 


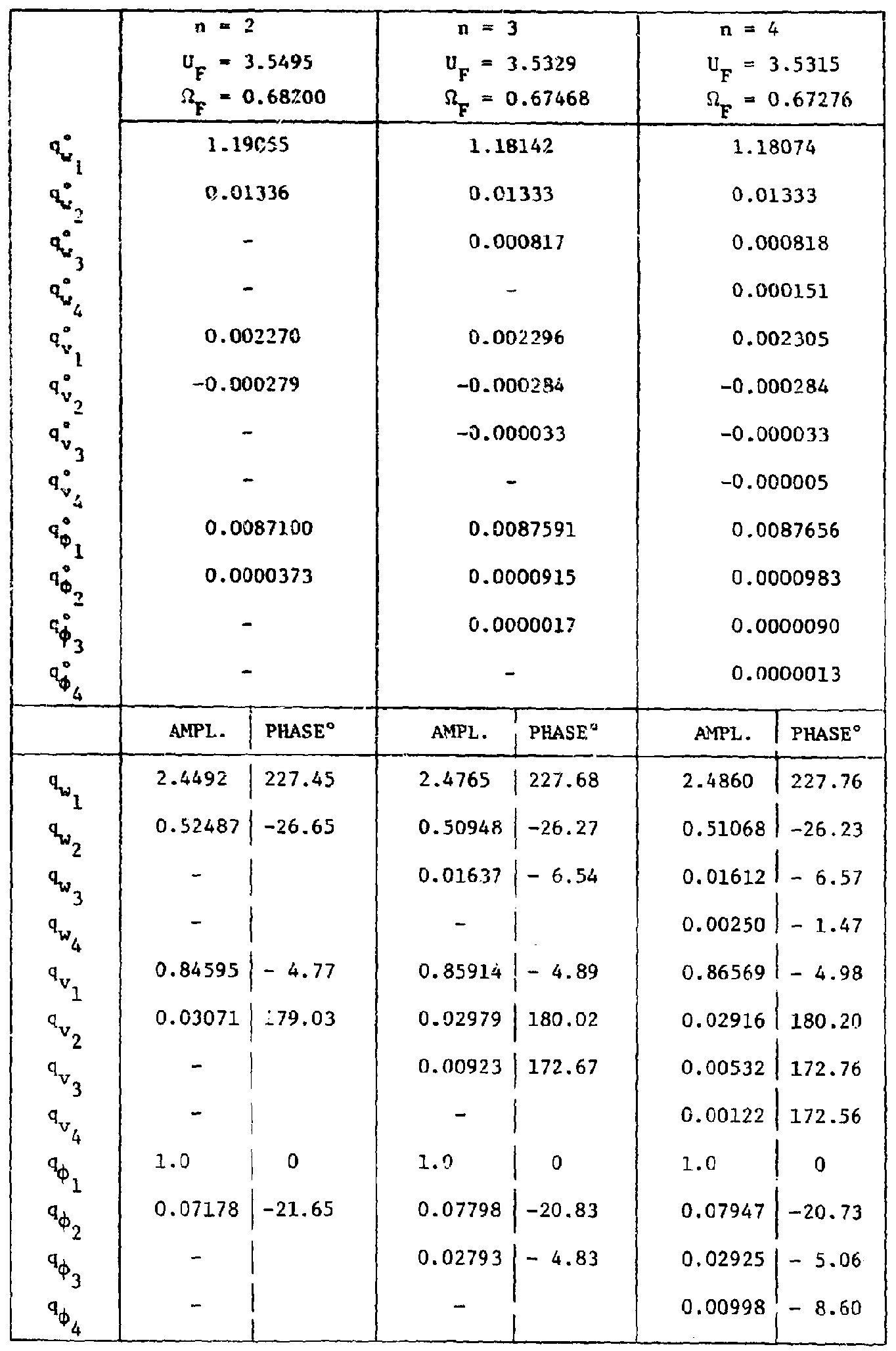

TABLE 4.3 Modal Convergence for Flutter at Steady Lift for $\alpha=0.01$ rad. $\left(M=9.4, P=0.01, i_{\alpha}=0.25, A=0.1, S=0.1, \tau=25, C=0\right)$ 


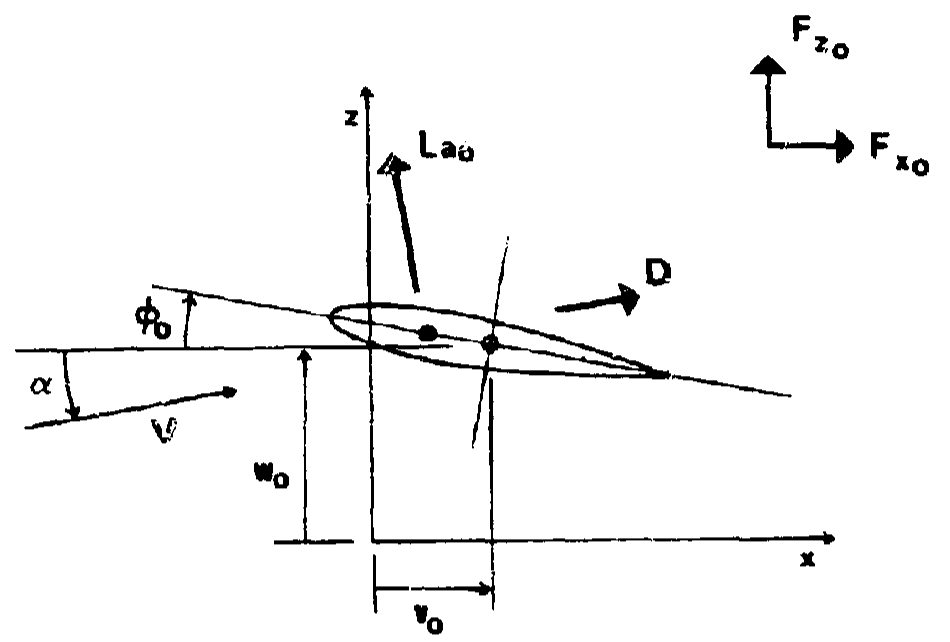

FIGURE 4-1 Resolution of Applied Steady Loads into Components $F_{x_{0}}, F_{z_{0}}$

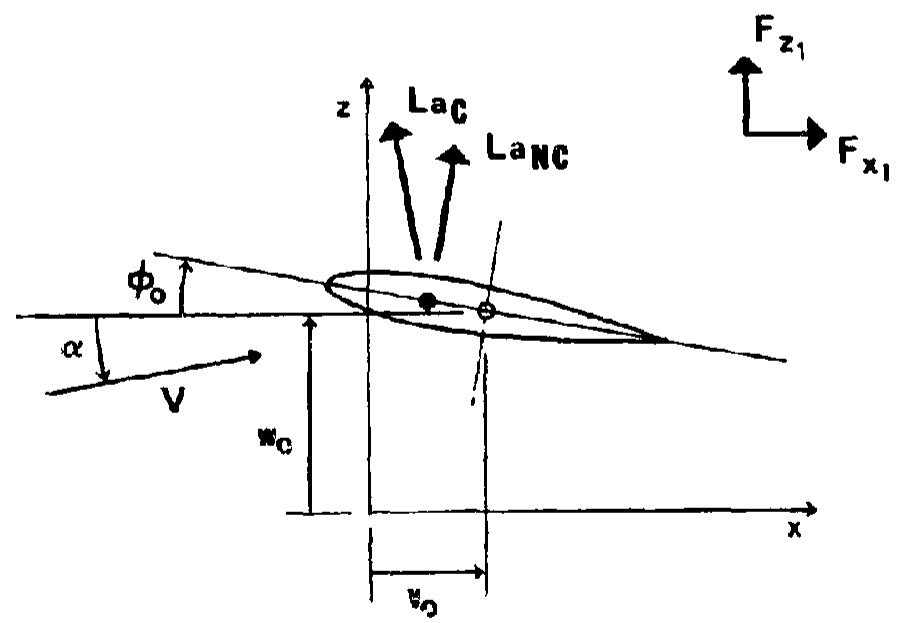

FIGURE 4-2 Resolution of Unsteady Alrloads into Components $\mathbf{F}_{\mathbf{x}_{1}}, F_{z_{1}}$ 


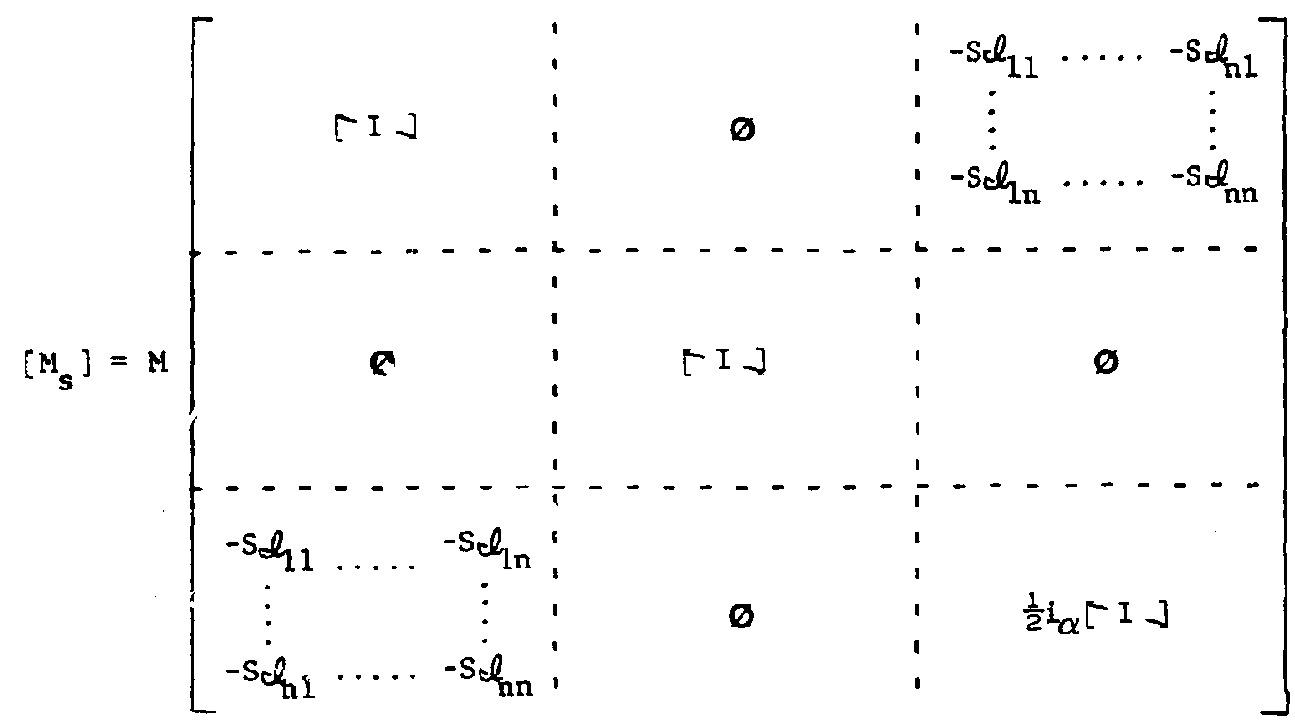

ABBREVIATIONS OF SUMMATIONS APPEARING IN $\left[\mathrm{K}_{\mathrm{s}}\right]$ :

$$
\begin{aligned}
& R_{i j \phi \phi}=\sum_{\mu=1}^{n} \sum_{v=1}^{n} R_{i j \mu v} \cdot q_{\phi_{\mu}}^{\circ} q_{\phi_{v}}^{o} \\
& \mathrm{R}_{w i \phi j}=\sum_{\mu=1}^{\mathrm{n}} \sum_{v=1}^{\mathrm{n}} \mathrm{R}_{v i \mu \mathrm{j}} \mathrm{q}_{\phi_{\mu}}^{\circ} \frac{\mathrm{q}_{\mathrm{b}}^{\mathrm{o}}}{\mathrm{o}} \\
& \mathrm{H}_{\phi i j}=\sum_{v=1}^{n} \mathrm{H}_{v i j} q_{\phi}^{\circ} \\
& \mathrm{H}_{i w j}=\sum_{v=1}^{n} \mathrm{H}_{i v j} \frac{\mathrm{q}_{\mathrm{w}}^{0}}{\mathrm{~b}}
\end{aligned}
$$

FIGURE 4-3(a) liass and Stiffness Matrices Appearing in Eouation $(4-19)$ 


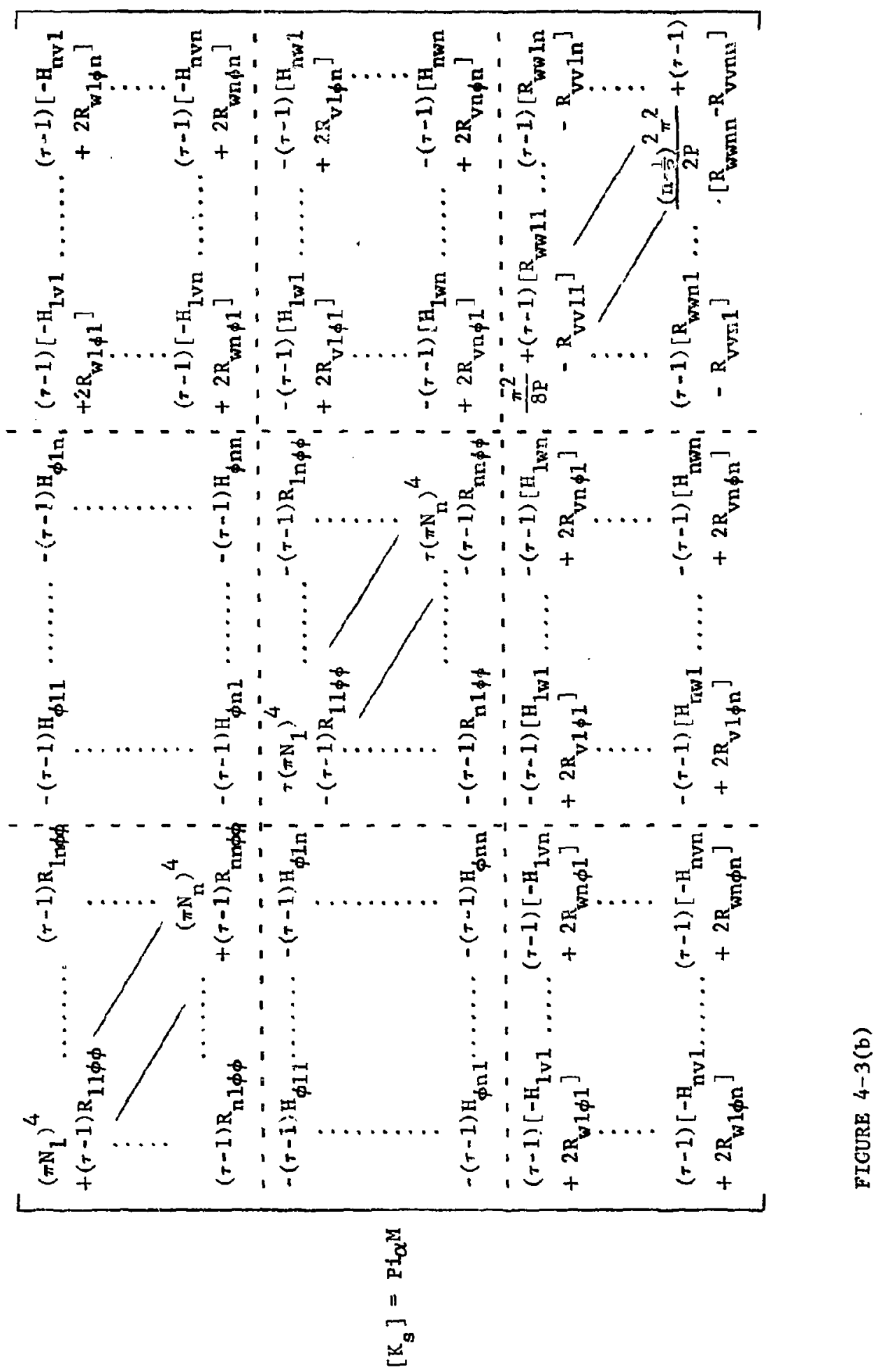




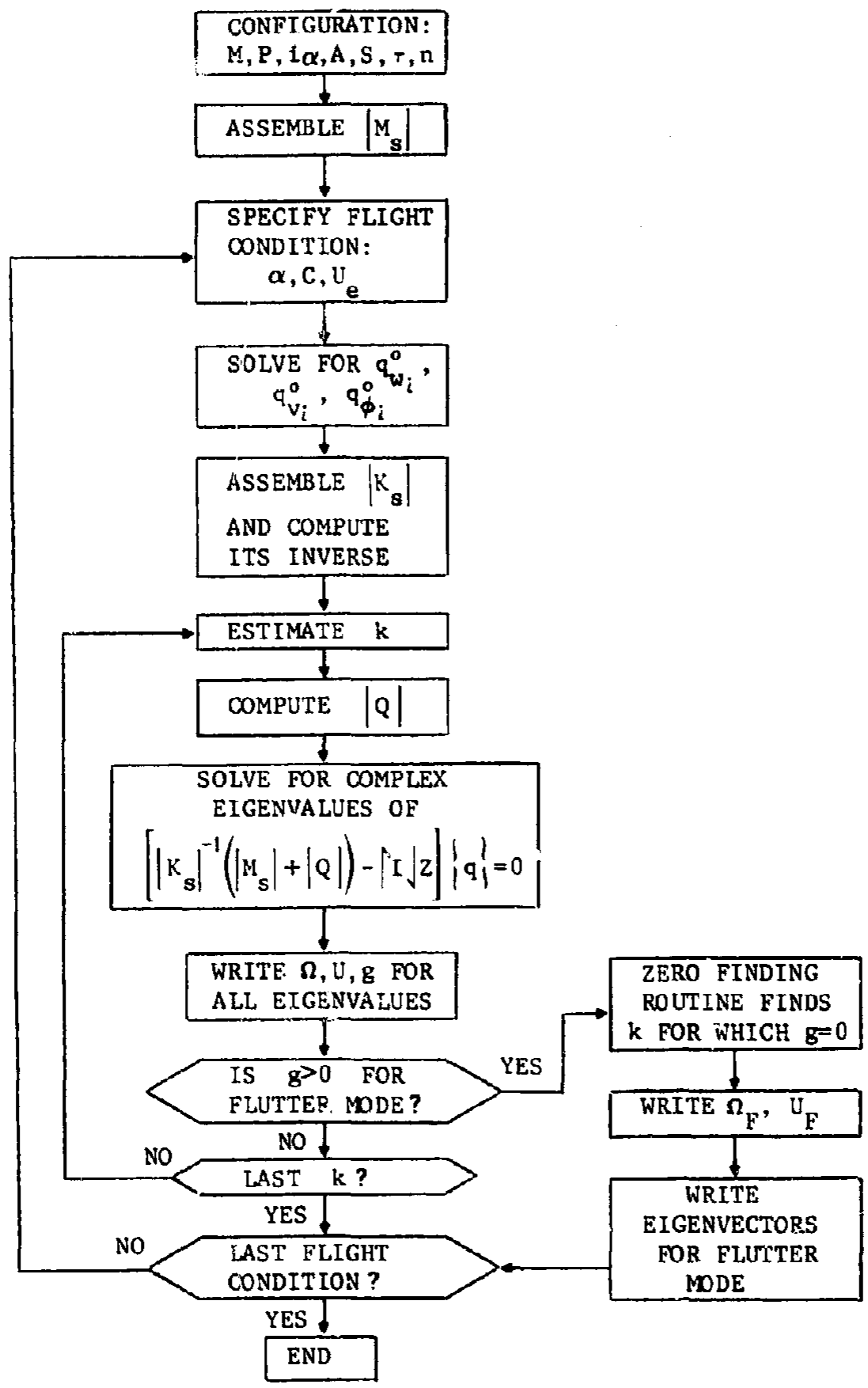

FIGURE 4-4 Flow Chart for Solution Procedure Using V-g Method 


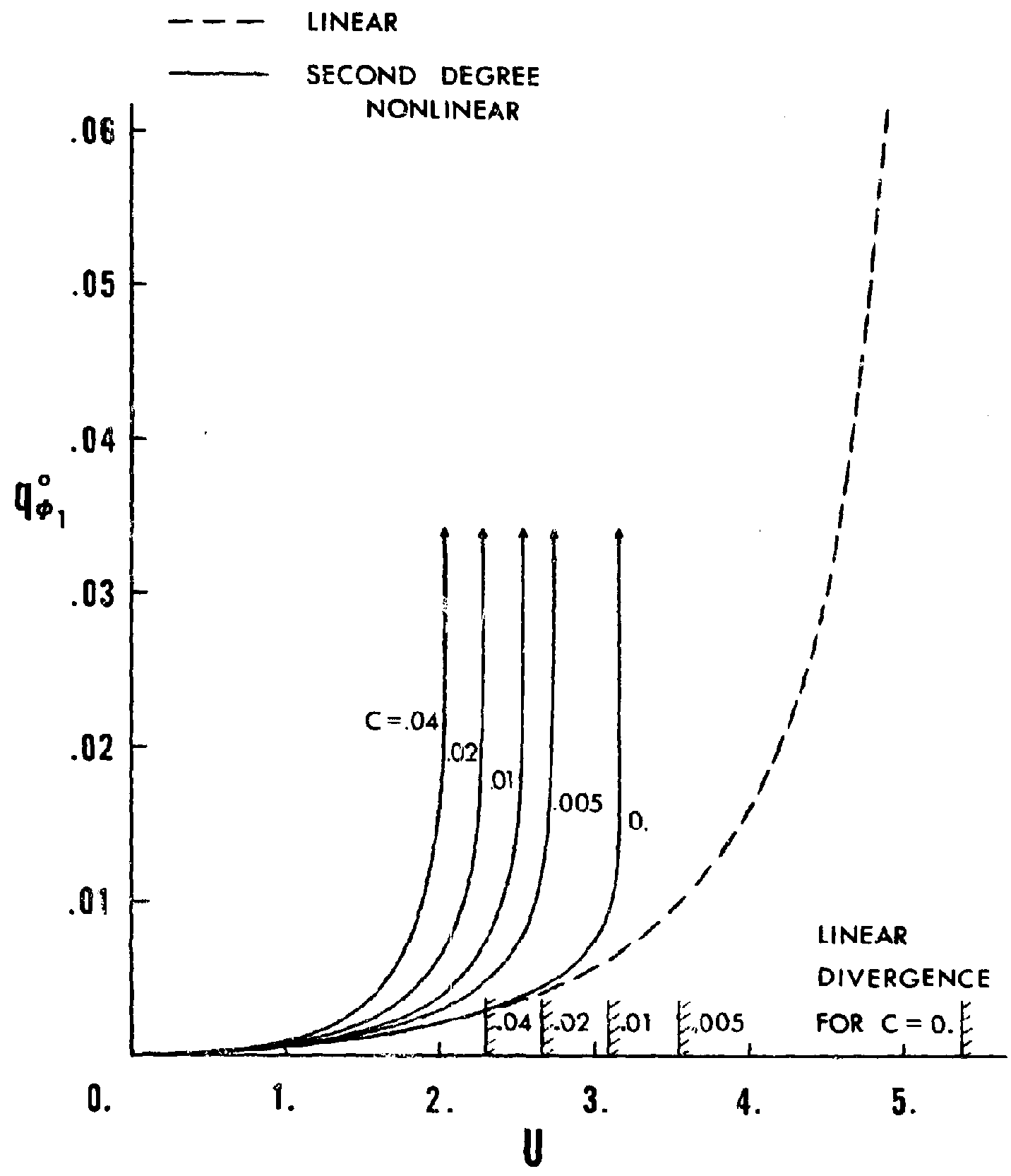

FIGURE 4-5 Deflection of the First Torsion Assumed Mode for $\alpha=.01$ and Increasing Speed, Comparing LInear and Second-Degree Nonlinear Solutions. $\left(M=9.4, F=0.01, i_{\alpha}=0.25\right.$, $\mathrm{A}=0.1, \mathrm{~S}=0.1, \mathrm{~T}=25$, and $\mathrm{n}=3$ ) 


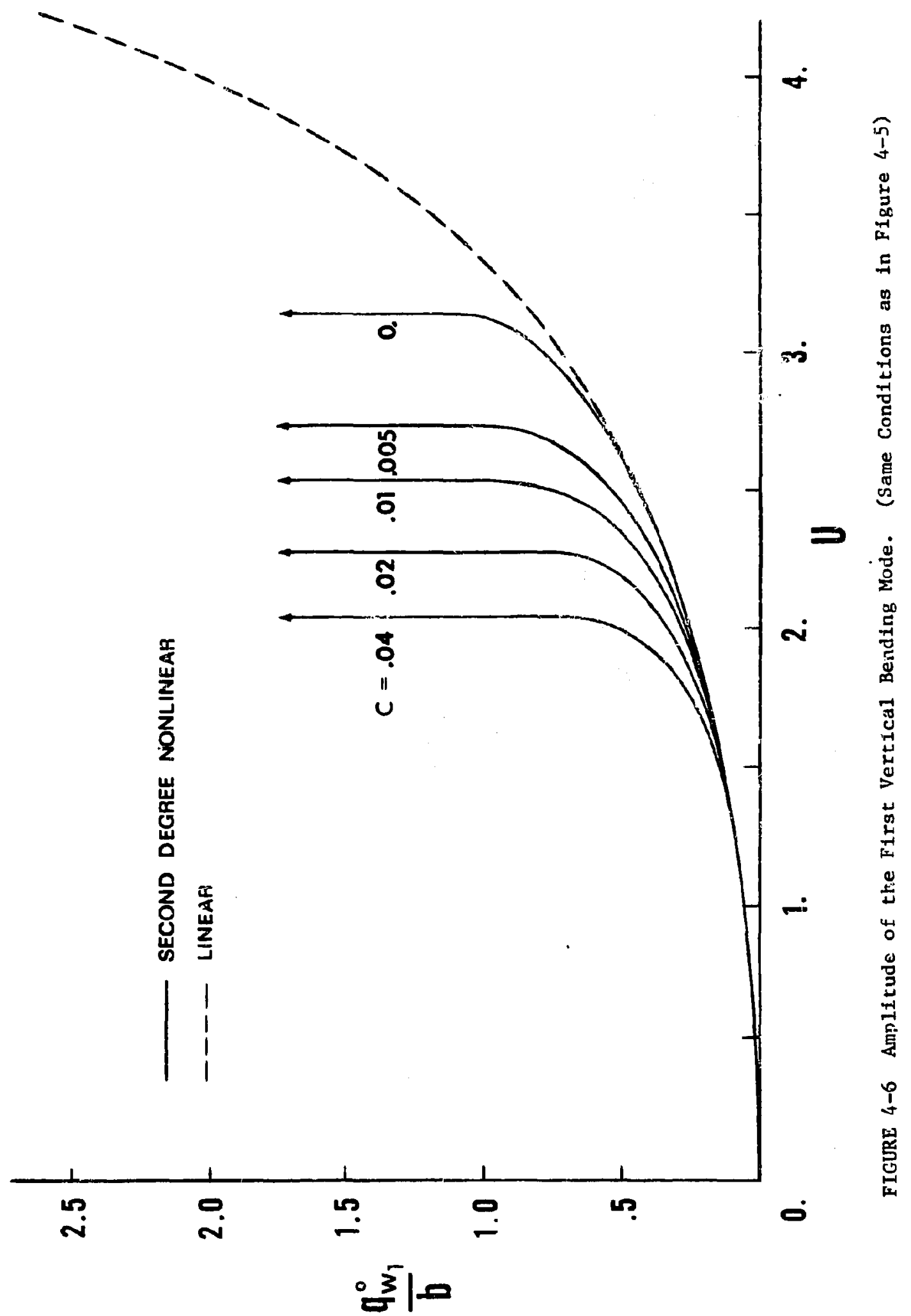




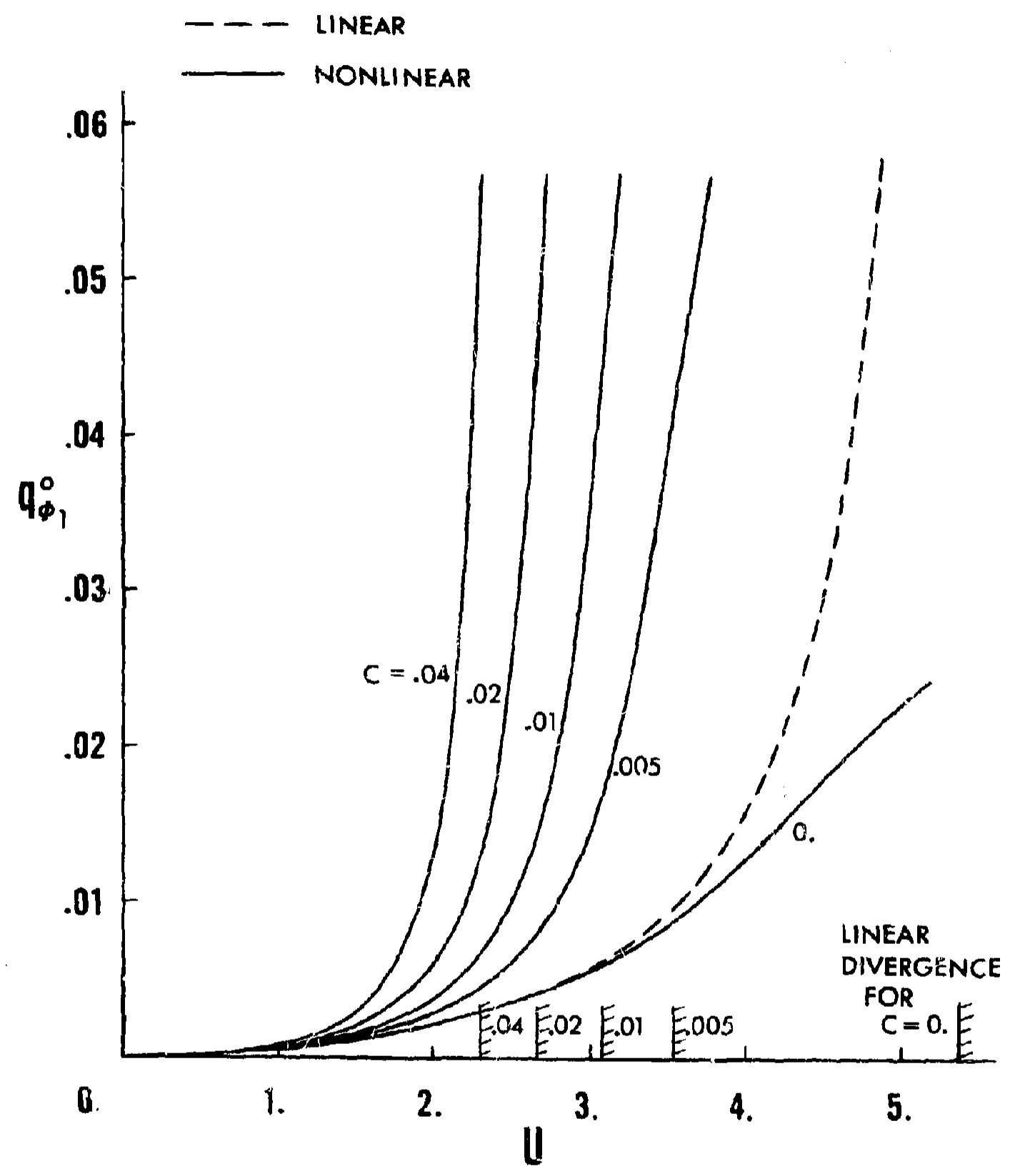

FIGIRE 4-7 Amplitude of the First Torsion Mode for $\alpha=0.01$ and Increasing Speed, Comparing Linear and Full Nonlinear Solutions. $\quad\left(N_{i}=9.4, P=0.01, i_{\alpha}=0.25, A=0.1, S=0.1\right.$, $\tau=25$, and $n=3$ ) 


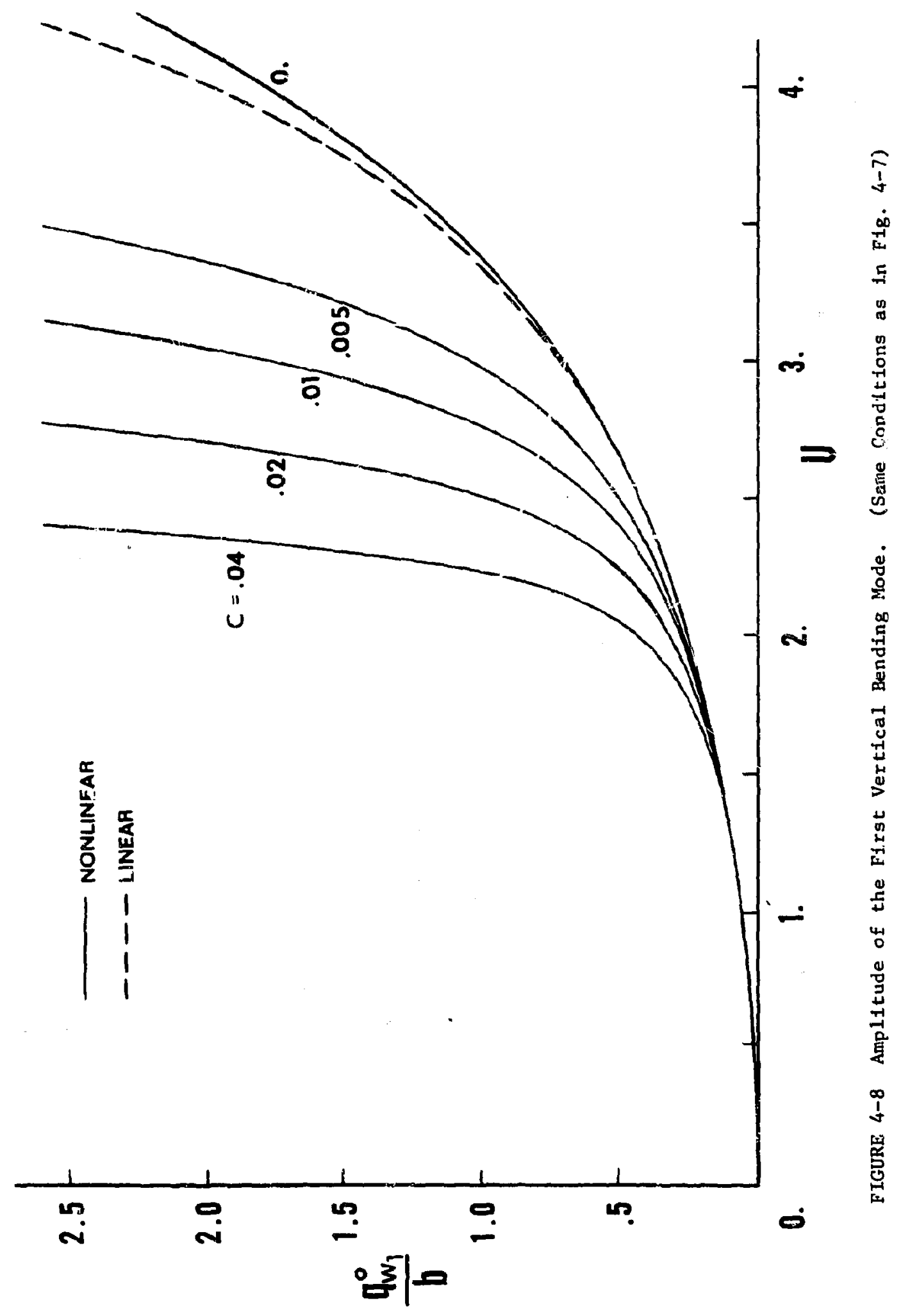




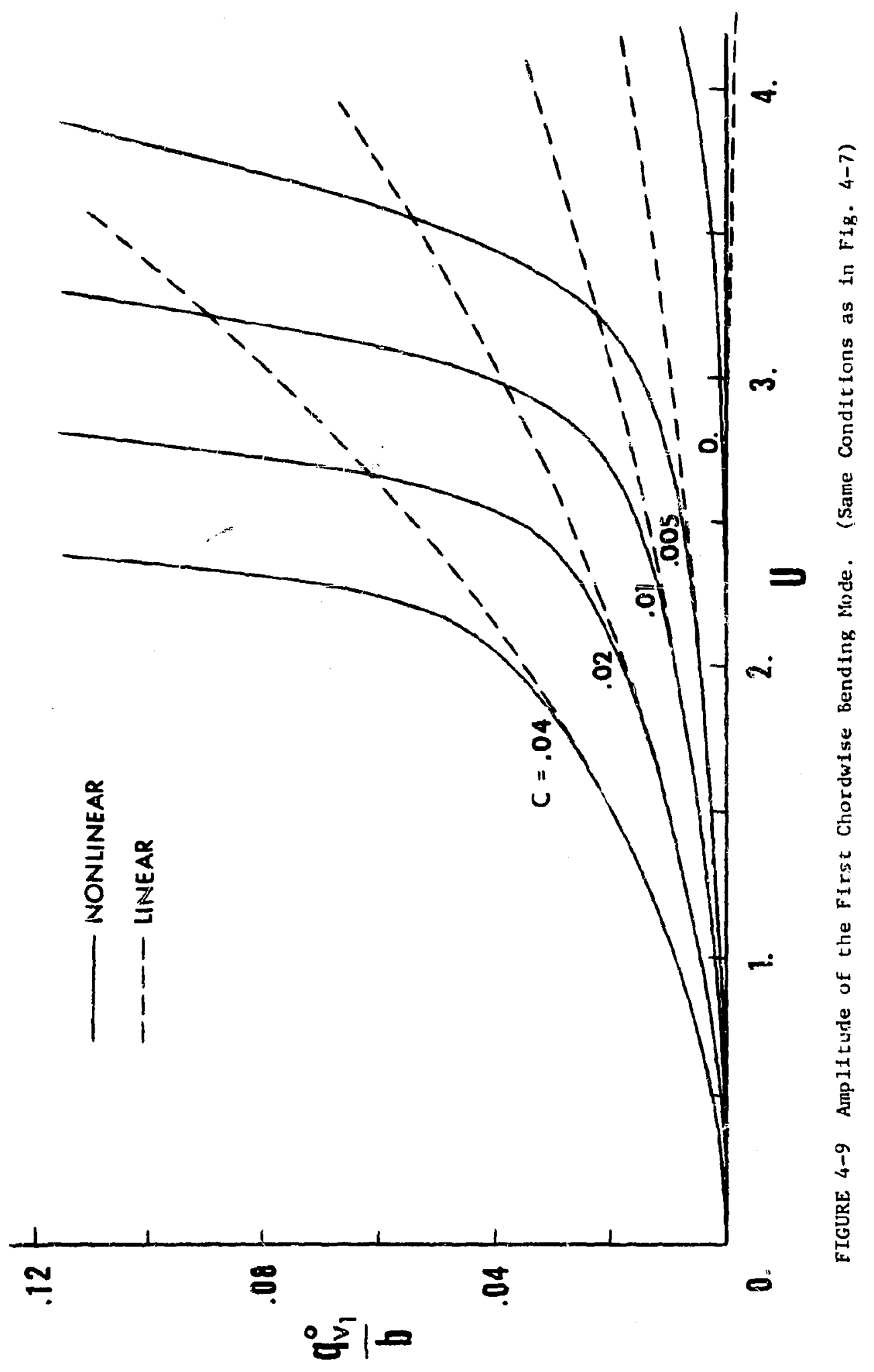




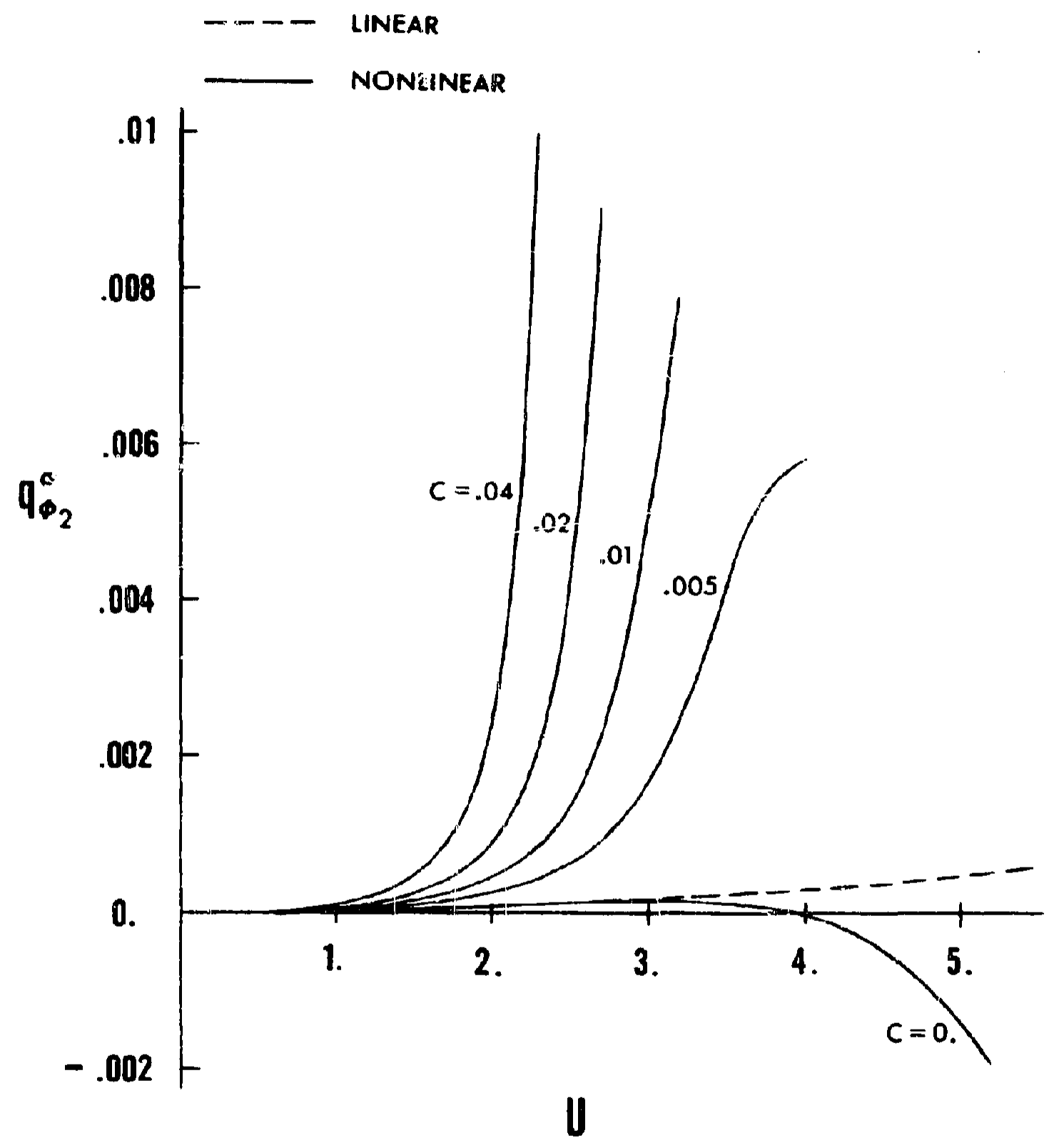

Figure 4-10 Amplitude of the Second Torsion Mode. (Same conditions as in Fig. 4 - 7) 


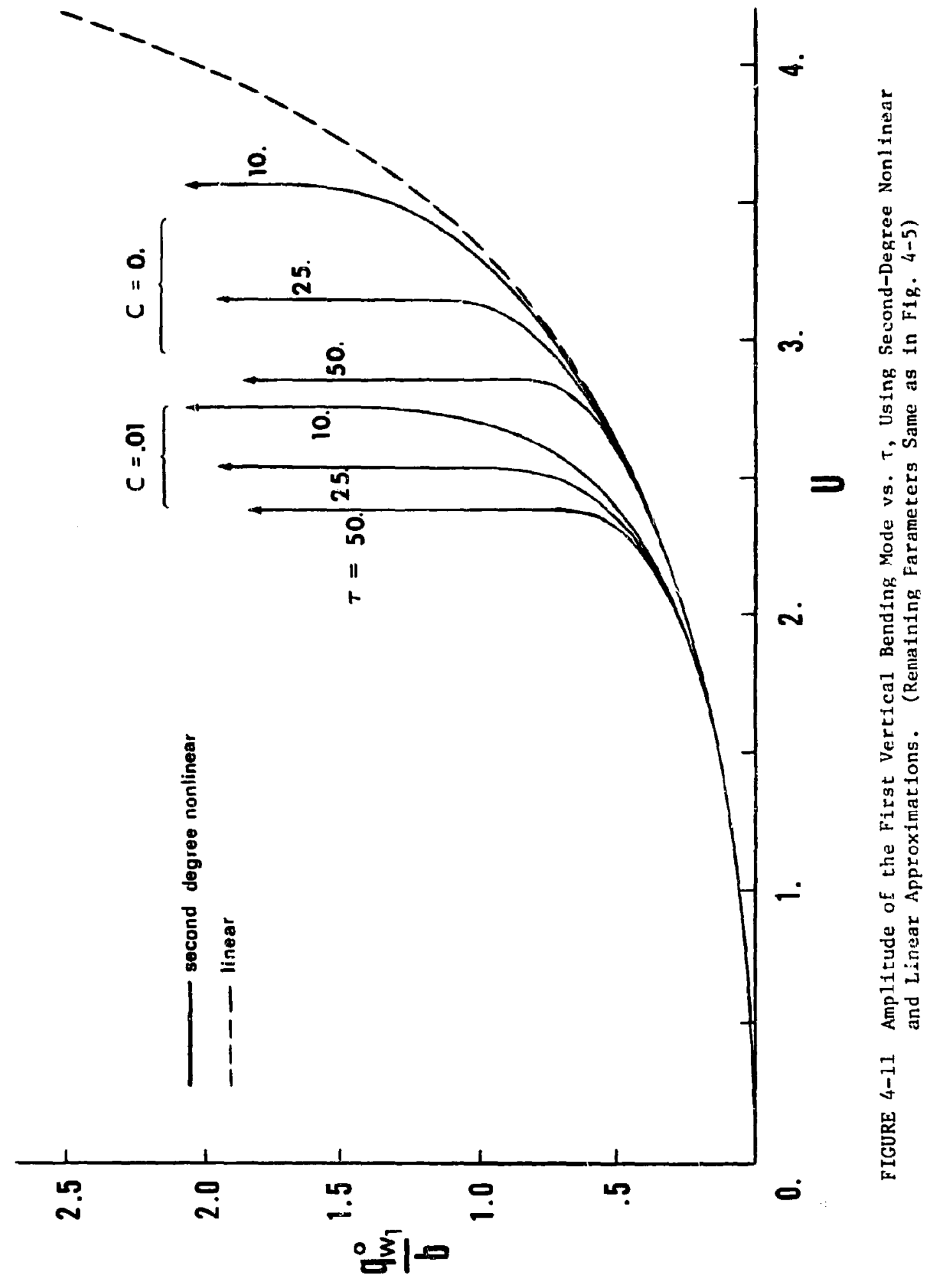




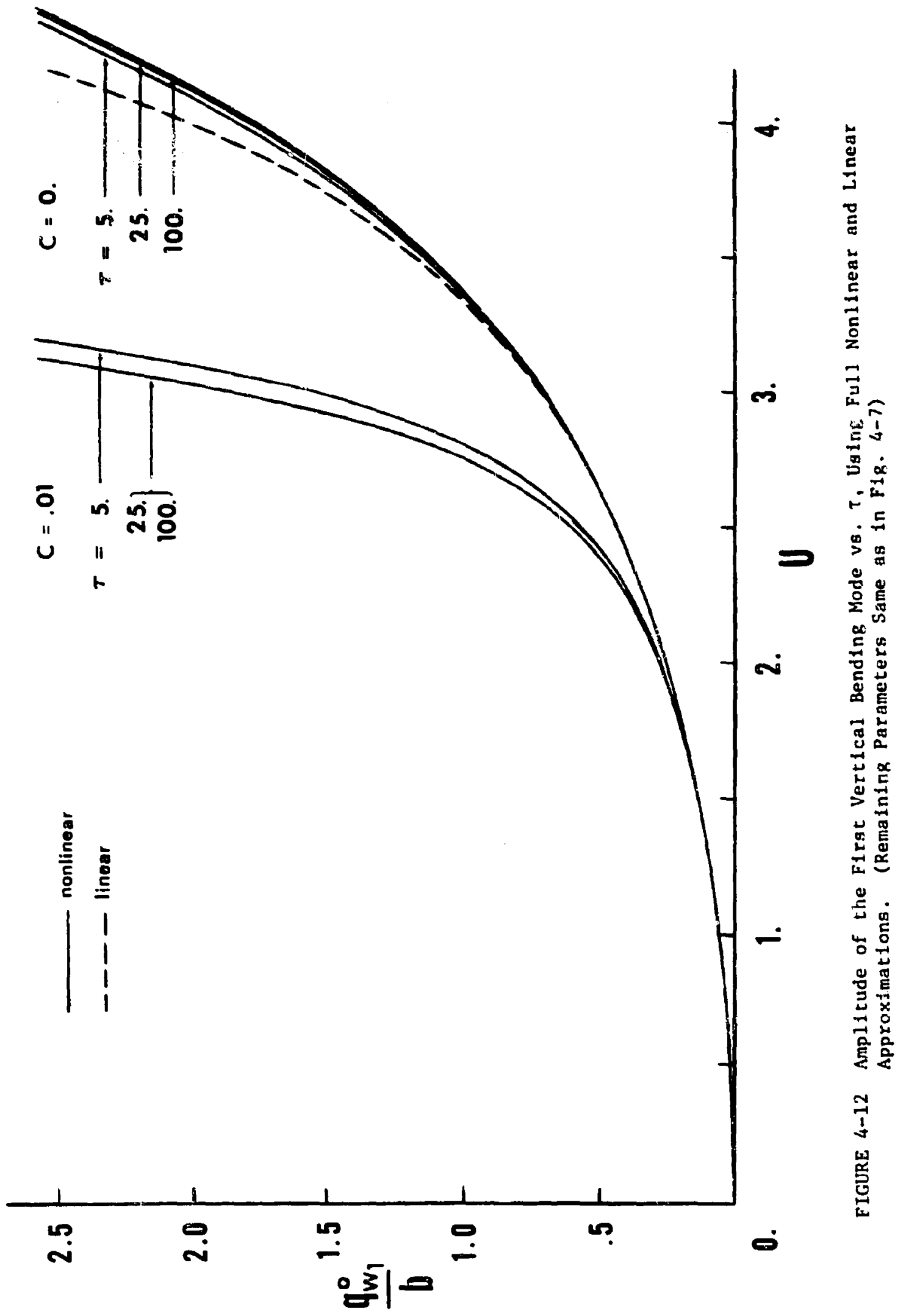




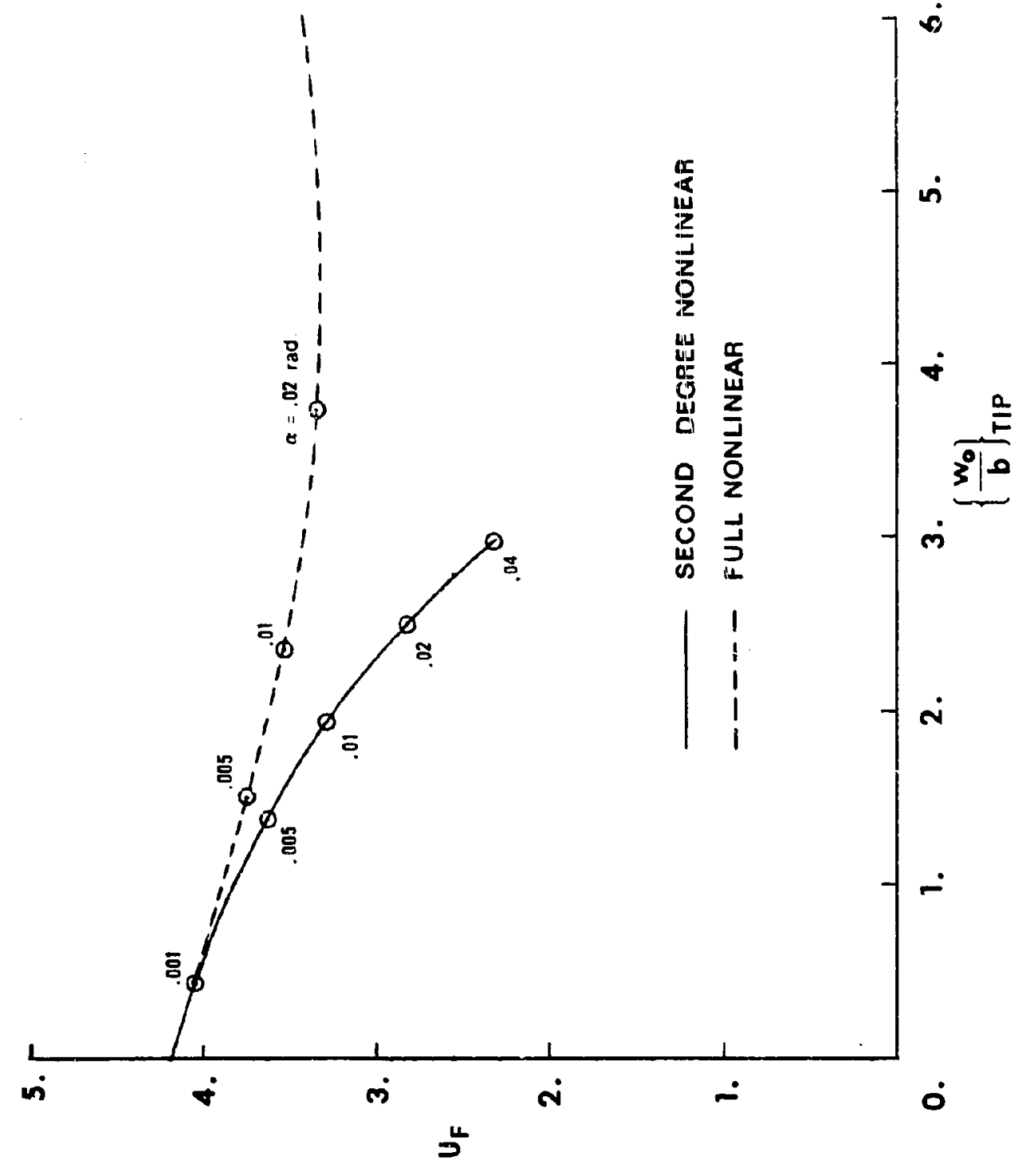

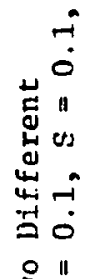

$3<$

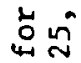

웅

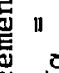

空

品

तें

에

F

Ẽ்

즌 11

ปู

㟧

需

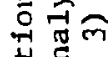

Uै।

急

ชู $E$ 范

눙

过

की 0

ปู

岁虽"

死安

告 


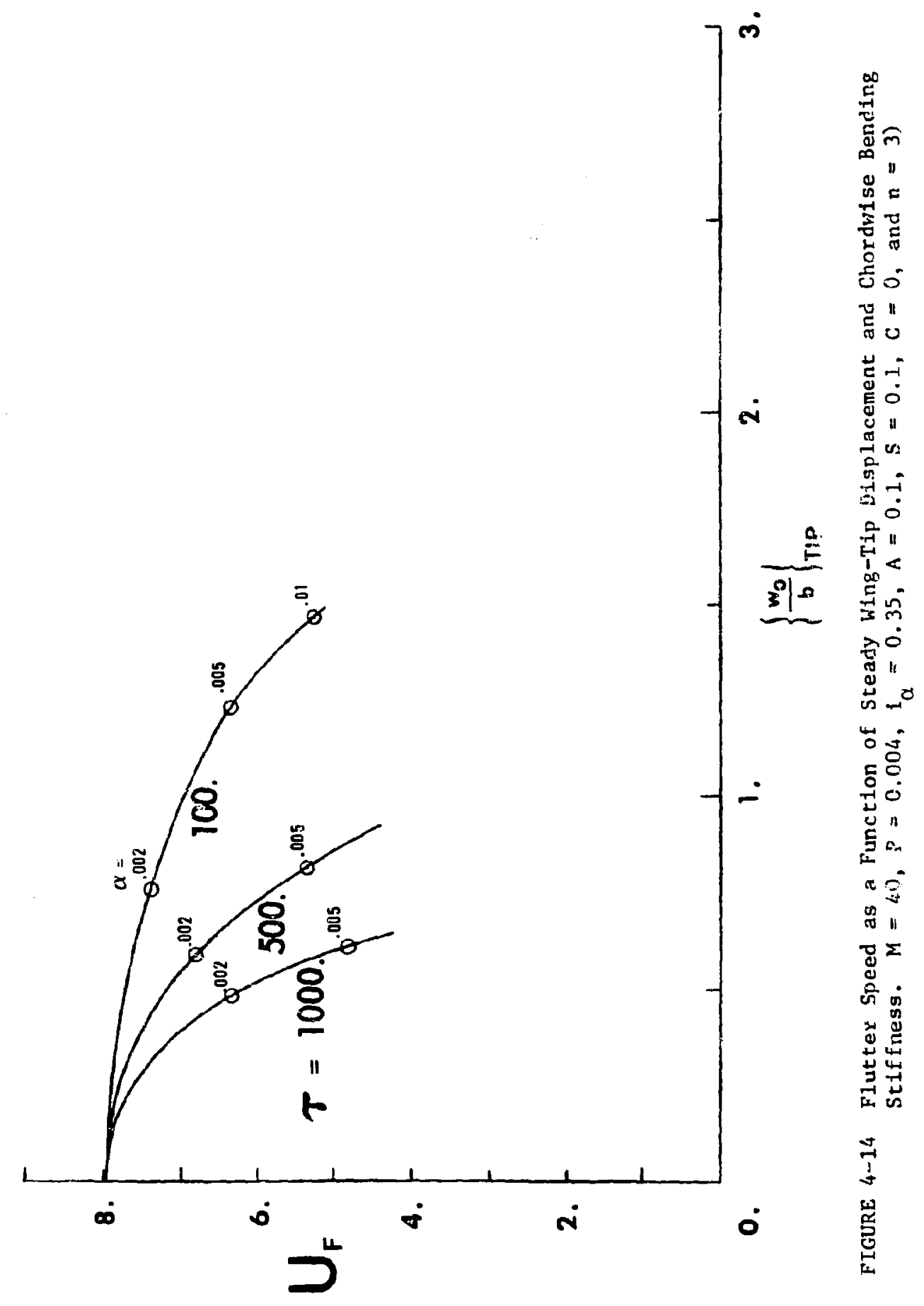




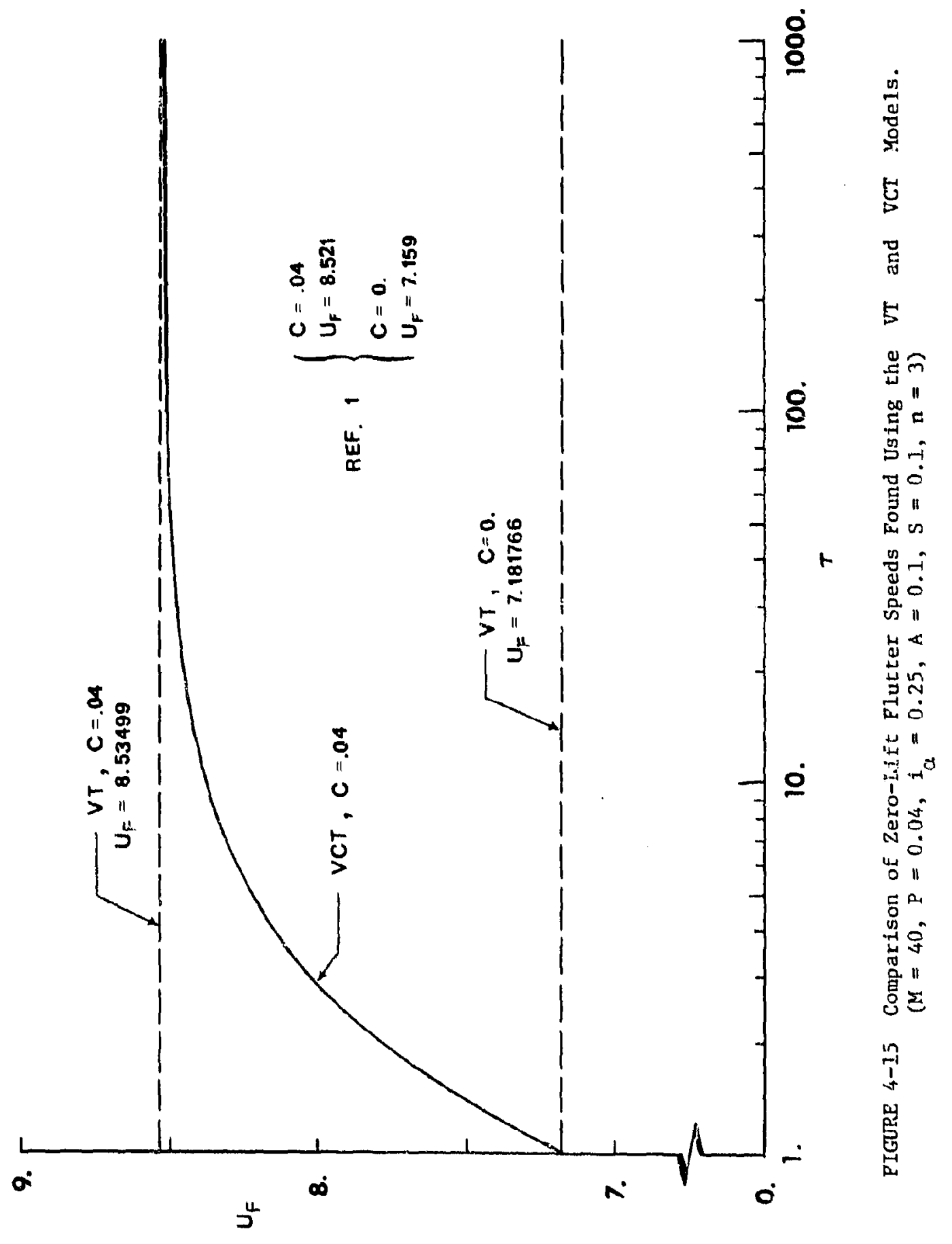


Chapter V

THE DETERMINATION OF AERCEIASTIC MODES FOR ARBITRARY VELOCITY

\section{A. Incompressible Strip Theory Airloads for Arbitrary Motion}

The procedure for determining flutter velocity developed in Chapter IV has several drawbacks. It requires a matched point analysis in which an estimated velocity $U_{e}$ used to calculate steady deflections has to be iteratively matched to the lowest calculated flutter speed $\mathrm{U}_{\mathrm{F}}$ for the proper structural damping. Consequently the solution for a stability boundary over a range of lifting conditions can be lengthy. Furthermre, intermediate computations have no physical significance and are if limited qualitative value. The behavior and degree of stability of Individual aeroelastic modes, which becomes more interesting with the addition of the fore-and-aft bending degree of freedom, has proven to be difficult to deduce from the $\mathrm{V}-\mathrm{g}$ solutions. The only quantitative information available pertains to the neutral stability conditions found for the mode which experiences flutter.

We would like to have physically meaningful information regarding dynamics of the system at any desired speed. That is, we would Iike to know the complex eigenvalues of the aeroelastic modes at subcritical and supercritical velocities. Obviously a major drawback of the $V-\varepsilon$ method is its dependence upon simple harmonic air loads.

Several solution procedures were studied, which replace the Fourler transformation with respect to $t$ by Laplace transformation with respect to $t$ and obtain at least an approximation to the modal stability below and above the flutter velocity. The p-k method, a

91

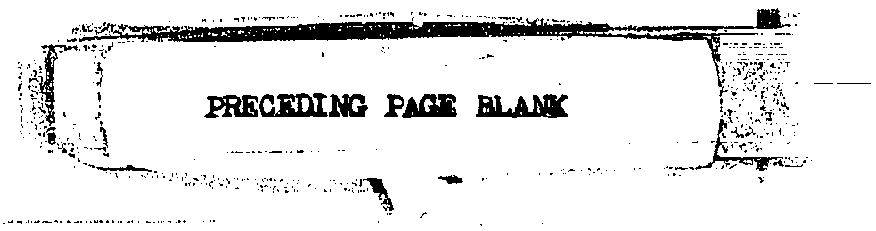


British flutter analysis technique (Ref. 8), uses the same simple harmonic airloads but assumes that these loads are approximately complex elgenvalues $s=\sigma+1 \omega$, where $\omega$ is the frequency used in calculating the airloads and $|\sigma| \ll|\omega|$.

Another approach, commonly applied in helicopter blade stability analysis, involves use of quasisteady aerodynamic theory by assuming $k$ is small enough to permit $C(k) \approx 1$. Laplace transformation of the system then yields only linear and quadratic terms in $s$. Linear matrix techniques can then be used in determining the roots. This level of approximation neglects entirely the effect of the unsteady wake upon the circulatory airloads and is not apnropriate for the magnitudes of reduced frequencles observed in many flutter calculations by the $\mathrm{V}-\mathrm{g}$ method.

A third possible course is to apply an augmented-state method, which approximates the actual unsteady aerodynamic loads for arbitrary motion with a transfer function relating airfoll displacements to loads having a rational Laplace transform, resulting in a linear matrix eigenvalue problem for the aeroelastic modes. Goland and Luke (Ref. 9) used this route to study wing bending-torsion flutter. They adopted the R.T. Jones (Ref. 10) approximation to the Wagner indicial lift Function to express unsteady airloads in rational form, taking the Laplace transform in time. In additson to their accurate description of the bas:c kending-torsion aeroelastic behavior at all flight speeds, Goland and Luke demonstrated that the severity of flutter cannot be reliably inferred from solutions by the $V$-g method. 
Each of the techniques mentioned above atcempts to gain information about aeroelastic modes whose elgenvalues have nonzero real parts. The true effect of the unsteady wake on the aerodynamic loads for arbitrary motions is approximated to varying degrees. This is done quite well for most motions in the case of augmented-state methods, marginally in the $p^{-k}$ method, and not at all In the quasisteady case.

For present purposes all of these schemes were rejected in favor of the more exact approach developed by Edwards (Ref. $11^{*}$ ). An important contribution of Ref. 11 (adapted from Scars, Rei. 21) is the definition of a generalized Theodorsen function to represent the exact circulatory two-dimensional incompressible unsteady airloads in the Laplace domain for arbitrary motions. The generalized Theodorsen function is expressible in terms of the modified Bessel functions of complex argument $K_{0}$ and $K_{1}$ as

$$
c(\bar{s}) \equiv \frac{K_{1}(\bar{s})}{K_{0}(\bar{s})+K_{1}(\bar{s})}
$$

where

$$
\bar{s}=\frac{s b}{V}
$$

Although previous investigators had recognized that this form was convergent for the right half plane, representing divergent oscillatory motions with $\operatorname{Re}(s)>0$, the restriction on the integral definitions of the modified Bessel functlons caused some Investigators to believe that convergent oscillatory motions (s in the left half plane) could

\footnotetext{
${ }^{\star}$ See also Milne (Ref. 22).
} 
not be so represented. Edwards observed that $K_{0}(\bar{s})$ and $K_{1}(\bar{s})$ are defined and analytic throughout the s-plane except for a brarich point at the origin. When one places a branch cut along the negative real axis, $C(\bar{s})$ can be shown by analytic continuation to relate circulatory loads and displacements throughout the s-plane except along this cut. It is, in effect, an "aerodynamic transfer function" in the Laplace domain.

With substitution of $\vec{s}=i k$ in $(5-1)$, the familiar Theodorsen function of reduced frequency for simple harmonic motion is recovered. Although arbitrary motion is now being considered rather than simple harmonic motion, the two approaches yield similar forms when the initial conditions arising in the transforms are neglected. In fact, the simple harmonic airloads $(4-18)$ and $(2-3)$ can be used for arbitrary unsteady motion simply substituting $C(\bar{s})$ for $C(k)$ and $s$ for $i w$. The modified Bessel functions are computed from their ascending powex series expansions, as mentioned in Ref. 11 and described in Appendix B. Since the transforms of aerodynamic loads will be multiplevalued functions because of the branch point of $c(\bar{s})$ at the origin, the convention

$$
-\pi<\operatorname{Arg}(s) \leq \pi
$$

is used for the cut on the negative real axis. The generalized Theodorsen function is computed by Edwards in the form $c\left(\bar{r} e^{i \theta}\right)$ for representative values $0 \leq \theta \leq \Pi$. It is shown to approach 1 as $\bar{r}+0$ and $\frac{1}{2}$ as $\bar{r} \rightarrow \infty$ for a11 $\theta$. 
W1th the ablifty to compute unsteady two-dimensional incompressible airloads for arbitrary motions in hand, the simple harmonfc stability analysis developed in Chapter IV can be generallzed for this case. All of the true aeroelastic elgenyalues and eigenfunctions can be obtained for any prescribed speed $U_{e}$. Stability can be displayed with root locus dagrams. The solution technique is developed in the following section.

\section{B. Solution for the Aeroelastic Roots by Means of Assumed Modes}

Formally, the procedure for developing the modal equations needed for the true aeroelastic modes throughout the complex plane begins with Laplace transformation of the Iinearized equarions of motion (3-25). The transformed perturbation displacements are then expressed as series expansions in the assumed modes as

$$
\begin{aligned}
\bar{w}_{1}(y ; s) & =\sum_{i=1}^{n} \tilde{r}_{w_{i}}(y) \bar{q}_{w_{1}}(s) \\
(5-2) \quad \bar{v}_{1}(y ; s) & =\sum_{i=1}^{n} f_{v_{i}}(y) \bar{q}_{v_{1}}(s) \\
\bar{\phi}_{1}(y ; s) & =\sum_{i=1}^{n} f_{\phi_{i}}(y) q_{\phi_{1}}(s)
\end{aligned}
$$

A system of homogeneous, linear, algebraic equations in the generalized displacements $\bar{q}_{w_{i}}, \bar{q}_{v_{j}}, \bar{q}_{\phi_{i}}$ can then be derived by Galerkin's method, as before. Nontrivial solutions are given by the zeros of the determinant in $s$. Since the coefficients in this determinant which arise from the aerodynamic loads wili contain the nonrational function $C(\bar{s})$, this will not be a polynomial eigenvalue problem. Roots $s=\sigma+i \omega$ will thus have to be obtained by iteration. 
Since ful1 development of the modal equations is analogous to that for the simple harmonic case, the stabilfty determinant in 9 will simply be constructed directly from the modal equations for simple harmonic motion defined by $(4-12),(4-18)$, and $(2-3)$. One replaces $1 \omega$ by $s$, ik by $\vec{s}$, and eliminates $g$. The elements of the aerodynamic matrix given in (4-18) for simple harmonic loads become, for arbitrary motion,

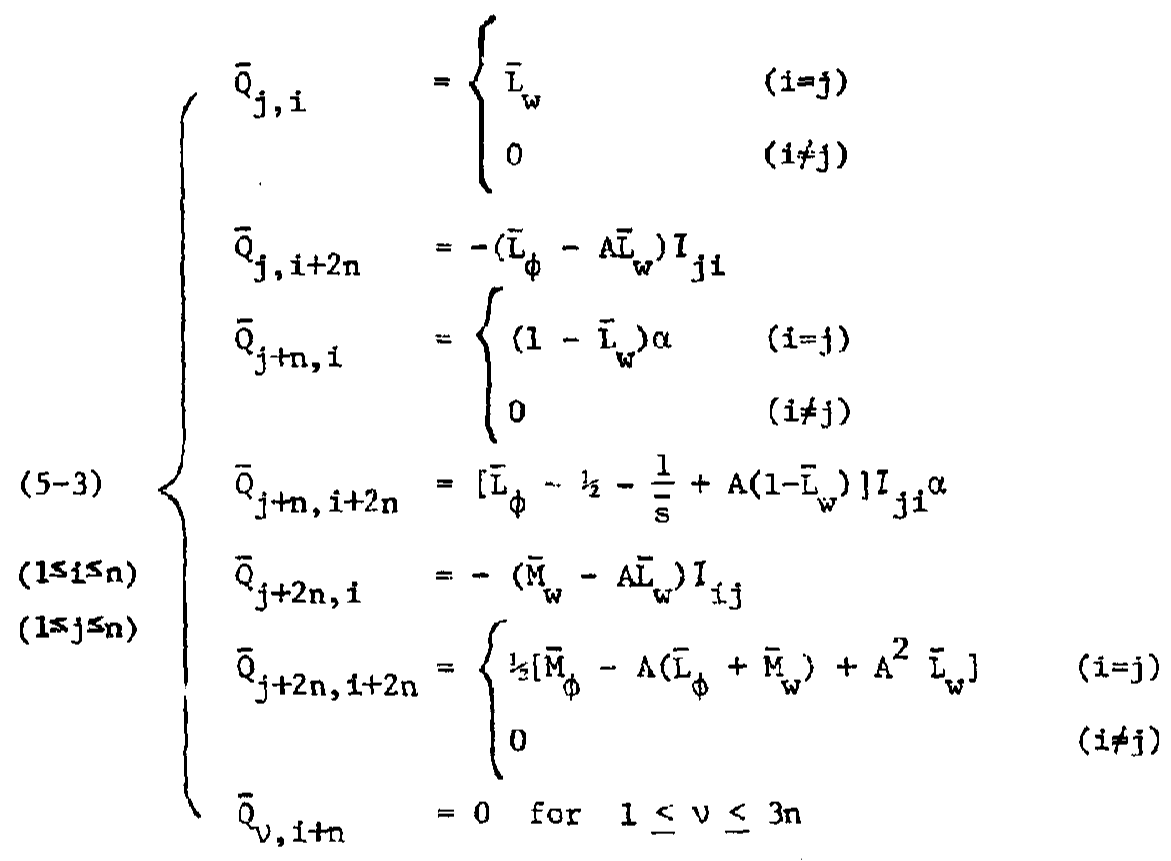

in which

$(5-4)\left\{\begin{array}{l}\bar{L}_{W}=1+2 \frac{C(\bar{s})}{\bar{s}} \\ \bar{L}_{\phi}=\frac{1}{2}+\frac{1}{s}[1+2 C(\bar{s})]+2 \frac{C(\bar{s})}{\bar{s}^{2}} \\ \bar{M}_{W}=\frac{\pi}{2} \\ \bar{M}_{\phi}=\frac{3}{8}+\frac{1}{s}\end{array}\right.$ 
These aerodyramic loads are based on the assumptions discussed prior to Eq. $(1-13)$.

With the same mass and stiffness matrices $M_{s}$ and $K_{s}$ illustraced In Fig. 4-3, and the aerodynamic matrix $\bar{Q}$ whose elements are defined by $(5-3)$, the matrix form of the rodal equations in $s$ becomes (5-5) $\left.\quad\left\{6\left[M_{s}\right]+[\bar{Q}]\right)+\frac{G I d}{J l^{2} s^{2}}\left[K_{s}\right]\right\}\{\bar{q}(s)\}=0$

(5-5) may the compared with the simple harmonic form (4-19). It is conventent to define a dimenstonless Laplace transform varlable

$$
p \equiv s \ell \sqrt{\frac{J}{G I}}
$$

which is related to the reduced laplace transform variable through the dimensionless velocity by

$$
(5-7) \quad \ddot{s}=\frac{s b}{V}=\frac{p}{U}
$$

The stability determinant thus takes the form

$$
(5-8) \quad\left|p^{2}\left(\left[M_{s}\right]+[\bar{Q}]\right)+\left[K_{s}\right]\right|=0
$$

Zeros of this determinant will yleld 3 n exact roots for the aeroelastic modes in terms of the $3 n$ assumed modes. These roots describe modal frequencies and stability at the speed $U_{e}$ used to calculate the steady-state deflections which enter as coeffictents in the stiffness matrix.

A computer program was developed to locate numerically the zeros of the determirant (5-8); the logic is outlined in Fig. 5-1. This 
format proved to be quite conventent for constructing root losus dagrams having efther the speed $U$, the root angle of atrack $\alpha$, or the drag parameter $C$ as the changing parameter. Any of the important aeroelastic modes, at any degxee of stability, could be traced through the complex plane as long as the tritial guess of $\bar{s}$ was sufficiently close to irs particular root locus.

Since the determinant order is $3 n$ and $n \geq 2$ js desirable to model adequately the physical system, numerical expansion of the determinant was not practical. A 1ibrary subroutine, employed to calculate the complex determinant, proved to be the source of numerical diffirulties. It was found that for $1 \leq \mathfrak{n} \leq 4$ and $\alpha=0$ the Fig. (5-1) program gave accurate results when compared to parallel V-g method neutral stability computations, agreeing to at least seven digits in flutter speeds. For $\mathrm{n}=2$ and $\alpha \neq 0$, which gives rise to steady deflections due to lift, similar good agreement was encountered. But for $n \geq 3$ and $\alpha \neq 0$, the prograin converged on zeros which did not match the neutrally stable $V-g$ predictions and were obviously incorrect from a physical standpoint. Subsequent investigation revealed that the nunerical difficulties originated in the library subroutine. When $\alpha=0$ the fore-and-aft bending degree of freedom is dynamically uncoupled, and the order of the determinant which was actually computed by the library subroutine was reduced to $2 \mathrm{n}$. Thus, for $\mathbf{n}=3$ and $\alpha=0$, the actual computed determinant was of order 6 , whereas for $n=3$ and $\alpha \neq 0$ the order was 9 . For the latter case the actual magnitude of computed determinants was of ten $0\left(10^{16}\right)$, while for $\alpha=0$ the $n=3$ determinants $0\left(10^{10}\right)$. For $n=2$ 
and $\alpha=0$ determanants again were $0\left(10^{10}\right)$. The magnitude of the computed determinsnt thus appears to be related to the numerical difficulties. Since $n=2$ results are judged to model the problem adequately and never encountered numerical problems, correction of the above difficulties was not pursued.

As a result, all root loci shown hereln for steady lifting, conditions involve $n=2$. As will be discussed, however, this regtriction does not compromise the modeling of the physical system nor prevent qualitative understanding of its behavior. Furthermore, numerical results always agree reasonably well when compared with $V-\xi$ computarions for $n=3, \alpha \neq 0$.

The algorithm used to estimate the zeros of the decerminant in the $\bar{s}$ plane, using an initial guess $\bar{s}_{o}$, is illustrated graphically in Fig. 5-2. The complex determinant is first calculated at $\bar{s}_{0}$ and at the two related points $\bar{s}_{0}+.001$ and $\bar{s}_{0}+1.001$. Points $A$ and $C$ are then determined, at which linear extrapolation in the two orthogonal directons predicts that the real part of the determinant vill vanish. Similarly poirts $B$ and $D$ are predicted, for which the imaginary part vanishes by extrapolation. A new guess for the root $\bar{s}_{1}$ is then determined as the intersection point of dashed lines in the figure. The process is repeaced until satisiactory convergence is realized. This simple scheme worked quite well and never failed to converge on a root, usually within four or five fterations. The convergence criterion used was generally $\| \bar{s}_{1}|-| \bar{s}_{0}|| \leq 10^{-5}$. Typical performance of che algorithm is documented in Table 5.1. 


\section{c. Mode Shapes for Aeroelastic Modes}

The mode shapes associated with roots determined for arbitrary motion by the determinant iteration method were conventently calculated with the same linear elgenvalue routine anpiled in solving the simple harmonic stability problen. For a root computed by treration, the nonrational aerodynamic temis containing $c(\vec{s})$ in the matrix $\overline{0}$ can be immediately evaluated. Thus one is led to a conventional matrix eigenvalue problem, which rotains the same root as one of irs eigenvalues and also provides its elgenvector. This approich worked well and, as a bonus, verified the accuracy of the roots compured by the determinant iteration scheme.

since numerical difficulties were encountered with the determinant evaluation routine for $n=3$ and $\alpha \neq 0$, this eigenvalue approach of rechecking its results for $n=2, a \neq 0$ (and for all $n$ with $\alpha=0$ ) is valuable. It offers the only means of verifying computed roots lying off of the iw axis. Correlation to at least six significant digits was always observed. Moreover, the accuracy of the $n=2$. $\alpha \neq 0$ determinant iteration solutions has been checked for a few representative cases by letting $n=3$ in the eigenvalue routine, with the known $n=2$ root as a first guess to evaluate the aerodynamic loads, and iterating until the true $n=3$ root is obtained. Two cases for wilch this was done, together with their eigenvectors, are shown in Table 5.1 , where $n=4$ roots are also given. The $n=2$ results obcainable by the determinant iteration routine are thus seen to be acceptably accurate even for the higher frequency mode. 
D. Inclusion of Unsteady Chordwise Loads Due to Leading Edge Suction

Since motion of the wing in fore-and-aft bending is permitted. unsteady chordwise loads car participate in the dynamic stability

problem. Unsteady two-dimensional incompressible airloads given in

Eqs. (4-18) and in (5-3) are strictly based upon the assumption that

the instantaneous resultant unsteady lift on anv diffoll section along.

the span is always perpendicular to the direction of the free stream

velocfty. Two-dimensional incompressiole potential flow theory, however, does predict an unsteady leading edge suction force which arises from the inverse squar a root singularity of the vorticity distribution along the airfoil chord at its leading edge. This effect will be included into the analysis within the framework of the linearized unsteady perturbation theory used to deternine stability. The effect of the unsteady propulsive force on stability can then easily be isolated by comparison of roots computed for the (4-18) airloads jirectly with roots determined with the airloads derived in this section.

The existence of a leading-edge surtion force due to the leadingedge sitizularicy was determined by von karman and Sears (kef. 12). Greenberg, (Ref. 13) in developing the propulsive force on an airfoil in an escillating stream, staces that a propulsive forie acting on the airfoil in the upstream direction.

(5-9) $\quad F_{S}=\Pi o C_{F}^{2}$

arisels from the unsteady vorticity distribution which behaves at the leading edge as

$$
\left.\gamma\right|_{\tilde{x} \rightarrow-1}=\left.\frac{2 C_{F}}{\sqrt{b} \sqrt{\tilde{x}+1}}\right|_{\tilde{x} \rightarrow-1}
$$


Ir this application the coefficient $C_{F}$ will contain contributions from the superposed vorticity distributions due to the steady and the unstendy lifting flow fields. Therefore $C_{F}$ will be a sum of steady and unsteady parts, and $C_{F}^{2}$ will involve a steady portion, a linear unsteady cross multiplication part, and a nonlinear quadratic unsteady term. In the context of the linearized unsteady perturbation approach to stability analysis, only the cross multiplication term will enter the dynamic equations. To include consistently the nonlinear unsteady propulsive force effect on stability, the nonlinear structural coupling ierms discarded during linearization would have to be reintroduced. As a consequence of Iinearization, the unsteady propulsive force can be included only when both steady and unsteady vorticity distributians are present. Thus the case of zero steady lift will have no contribution due to this effect to the state of stability. The effect will become increasingly pronounced as the steady lift is incieased. Tise vorticity singularity strength $C_{F}$ in (5-9) was given by Garrick (Ref. 14) for an airfoil oscillating in a uniform stream as

$$
c_{F}=\sqrt{2 b}\left\{\left[\dot{h}+v \alpha+b \dot{\alpha}\left(1_{\Sigma}^{1}-a\right)\right] c(k)-1 / b \dot{\alpha}\right\}
$$

with in positive downward. Converting to present notation, introducing the generalized Theodorsen function, and introducing superposition of sready and unsteady deflections gives

$$
\begin{aligned}
& \alpha+\alpha+\phi_{0}+\phi_{1} \\
& h+-w_{0}-w_{1}
\end{aligned}
$$


$(5-12)$

$$
C_{F}=\sqrt{2 b}\left\{\left(\alpha+\phi_{0}\right) v+\left[-\dot{w}_{1}+\phi_{1} v+b\left(\xi_{2}-a\right) \dot{\phi}_{1}\right] c(\bar{s})-{ }^{1} b \dot{\phi}_{1}\right\}
$$

The propulsive force $F_{s}$, using (5-9), is

$$
\begin{aligned}
F_{s}= & 2 \pi \rho b\left\{\left(a+\phi_{0}\right)^{2} v^{2}+2 v\left(a+\phi_{0}\right)\left[\left(-\dot{w}_{1}+\phi_{1} v\right.\right.\right. \\
& \left.\left.+b\left(s_{2}-a\right) \dot{\phi}_{1}\right) c(\bar{s})-1_{1} b \dot{\phi}_{1}\right]+\left[\left(-\dot{w}_{1}-\phi_{1} v+b\left(1_{s}^{1}-a\right) \dot{\phi}_{1}\right) c(\bar{s})\right. \\
& \left.-s_{s} b \dot{\phi}_{1}\right]^{2}
\end{aligned}
$$

The last squared term is the nonlinear time-dependent contribution and is neglected hereafter. The first squared term is the propulsive force on a flat-plate airfofl at incidence in steady flow. The resultant steady aerodynamic force should act at right angles to the free stream velocicy in potential flow, and this steady propulsive force can be interpreted physically as the component which tilts the resultant lift vector, obtained by sumning the pressure distribution at right angles to the chord, forward to become normal to the airstream.

The propulsive force can thus be seen to correct for the chordwise component of the lift which is compuced normal to the airfoil chord. The assumption incorporated into $(4-18)$ that the unsteady circulatory lift acts at right angles to the airstream must be discarded and the force assumed rather to be normal to the chord of the girfoil in its steady-state orfentation. The assumed direction of forces is shown in Fig. 5-3.

In the structure axis system, the loads are 
(5-14)

$$
\begin{aligned}
& F_{x_{1}}=-F_{s} \cos \phi_{0}+L_{n} \sin \phi_{0} \\
& F_{z_{1}}=L_{1} \cos \phi_{0}+F_{s} \sin \phi_{0}
\end{aligned}
$$

The following assumptions are made

$$
\begin{gathered}
\cos \phi_{0} \approx 1 \\
\sin \phi_{0} \approx \phi_{0} \\
F_{s} \sin \phi_{0}<L_{a}
\end{gathered}
$$

so that

$$
\begin{aligned}
& F_{z_{1}} \approx L_{a} \\
& F_{x_{1}} \tilde{\approx}-F_{s}+L_{a} \phi_{0}
\end{aligned}
$$

The ift for arbitrary motion in time to given by Ref. (5) as

$$
\begin{aligned}
u_{i 3}= & \operatorname{2\pi \rho bv}\left[-\dot{w}_{1} c(\bar{s})+\phi_{1} v c(\bar{s})+b\left(!_{2}-a\right) \phi_{1} c(\bar{s})\right] \\
& +\pi \rho b^{2}\left[-\ddot{i}_{1}+v \dot{\phi}_{1}-b a \ddot{\phi}_{1}\right]
\end{aligned}
$$

Combining (5-16) and the linear unsteady part of (5-13) into the second of $(5-1.5)$ y felds

$$
\begin{aligned}
\cdot(5-17) \quad \mathrm{F}_{\mathrm{x}_{1}}= & -4 \pi \rho v b\left(\alpha+s_{s}\right)\left(-\dot{w}_{1} c(\bar{s})+\phi_{1} v c(\bar{s})\right. \\
& \left.+\left(\left(L_{2}-a\right) c(\vec{s})-L_{2}\right) b \dot{\phi}_{1}\right]+\pi \rho h^{2} v \phi_{0} \dot{\phi}_{1} \\
& +\pi \rho h^{2} \phi_{0}\left[-\ddot{w}_{1}+v \dot{\phi}_{1}-b a \ddot{\phi}_{1} j\right.
\end{aligned}
$$


The loads can now be arranged into the unft generalized force format of (5-3) by Laplace transformation in time, substitution of the assumed modes as in (5-2), and formation of the generalized forces from relations such as (4-11). After this work, which is straightforward, the elements of the aerodynamic matrix which incisporate the linearized unsteady propulsive force are

$$
\begin{aligned}
\bar{Q}_{j+n, i}= & 2\left(1-\bar{L}_{w}\right) \alpha \delta_{i j} \\
& +\left(2-\bar{L}_{w}\right) \sum_{\nu=m+1}^{n} H_{v i j} q_{\phi_{\nu}}^{0} \\
\bar{Q}_{j+n, 1+2 n}= & 2\left[\overline{\mathrm{L}}_{\phi}-\frac{1}{2}-\frac{2}{\bar{s}}+A\left(I-\bar{L}_{w}\right)\right] I_{j i} \alpha \\
& +\left[\bar{L}_{\phi}-1-\frac{4}{\bar{s}}+\left(2-\overline{\mathrm{L}}_{w}\right) A\right] \sum_{\nu=m+1}^{n} Y_{j v i} q_{\phi_{\nu}}^{0}
\end{aligned}
$$

where the new modal integrals appear,

$$
Y_{i \cup j}=\int_{0}^{1} F_{w_{I}} I_{\phi_{V}} F_{\phi_{j}} d \tilde{y}
$$

The remainder of the terms of the aerodynamic matrix remain the same as in $(5-3)$.

Prior to actual calculations a further approximation is made. The terms in (5-18) which depend on sums of $q_{\phi_{\nu}}^{0}$ are neglected, eliminating the need to compute the $Y_{i v j}$. This is equivalent to assuming that the lift $L_{a}$ in Fig. 5-3 is aligned with the z-axis and that $\phi_{0}$ is neglected relative to $\alpha$ in the linear term of (5-13). Strictly, this simplification will alter the results somewhat, but it is not expected to change the overall effect of the propulsive force 
on stability and does simplify computations. The flrst order trend of the effect of $100 \%$ leading edge suction on stabllity ts the main point of interest and should not be affected.

The changes made to the aerodynamic matrix are therefore substitution of the terms

$$
\begin{aligned}
& \ddot{Q}_{j+n, i}= \begin{cases}2\left(1-\overline{\bar{L}}_{w}\right) a & (i=j) \\
0 & (1+j)\end{cases} \\
& (1 \leq j \leq n) \\
& \bar{Q}_{j \cdot n, i+2 n}=2\left[\bar{L}_{\phi}-\frac{1}{2}-\frac{2}{\bar{s}}+A\left(1-\overline{\mathrm{L}}_{w}\right)\right] I_{j i} \alpha \\
& (1 \leq 1 \leq x)
\end{aligned}
$$

for their counterparts in (5-3).

The program described in Section B includes the opticn of using either of these unsteady aerodynamic force systems, and a comparison of their relative effect on stability is made in the next chapter. Except for the $-\frac{2}{\bar{s}}$ term in the second of $(5-20)$, incidentally, the newer system simply involves doubling the magnitude of the terms in (5-3) that are replaced. 


$$
\begin{aligned}
& M=40, P=0.005, i_{\alpha}=0.25, A=0.1, S=0.1, \tau=25, \alpha=0, n=3, \\
& C=0, U=6.5
\end{aligned}
$$

\begin{tabular}{|c|c|c|}
\hline ITERATION & $\bar{s}$ & DETERMINANi AT $\bar{s}$ \\
\hline 0 & $-0.072968+10.0582071$ & $0.326 \times 10^{9}+10.1266 \times 10^{10}$ \\
1 & $-0.078290+10.067052$ & $-0.259 \times 10^{9}+10.504 \times 10^{8}$ \\
2 & $-0.079541+10.065839$ & $0.106 \times 10^{8}+10.504 \times 10^{7}$ \\
3 & $-0.079527+10.065910$ & $0.106 \times 10^{6}-10.178 \times 10^{6}$ \\
4 & $-0.079526+10.065910$ & $-0.281 \times 10^{4}+10.499 \times 10^{4}$ \\
\hline
\end{tabular}

$$
\begin{aligned}
& M=40, P=0.02,1_{\alpha}=0.25, A=0.1, S=0.1, \tau=25, \alpha=0.02, \mathrm{n}=2, \\
& C=0, U=7
\end{aligned}
$$

\begin{tabular}{|c|c|c|}
\hline ITERATION & $\bar{s}$ & DETERMINANT AT $\bar{s}$ \\
\hline 0 & $-0.0055179+10.119612$ & $0.261 \times 10^{11}-10.253 \times 10^{10}$ \\
1 & $-0.0014167+10.1074866$ & $0.125 \times 10^{10}+10.428 \times 10^{10}$ \\
2 & $0.0005501+10.1085285$ & $-0.459 \times 10^{7}+10.135 \times 10^{9}$ \\
3 & $0.0005027+10.1084861$ & $-0.129 \times 10^{6}+10.178 \times 10^{6}$ \\
4 & $0.0005026+i 0.1084861$ & \\
\hline
\end{tabular}

TABLE 5.1 Performance of the Determinant Iteration Algorithm for Two Cases, One Nonlifting With $\mathrm{n}=3$ and One at Steady Lift With $\alpha=0.02, n=2$ 
STEADY-STATE DEFLECTIONS:

\begin{tabular}{|c|c|c|c|c|c|}
\hline & $\mathrm{n}=2$ & $n=3$ & $n=4$ & & \\
\hline$q^{0} / b$ & 1.97768 & 2.01496 & 2.019088 & & \\
\hline & 0.022566 & 0.023221 & 0.023274 & $\mathbf{M}$ & $=40$ \\
\hline & - & 0.001453 & 0.001464 & $\mathbf{p}$ & $=0.005$ \\
\hline & - & - & 0.000271 & $i_{0}$ & $=0.25$ \\
\hline $\mathrm{q}_{\mathrm{y}}^{\mathrm{a}^{4} / \mathrm{b}}$ & 0.003170 & 0.003534 & 0.003594 & A & $=0.1$ \\
\hline $\mathrm{q}_{\mathrm{v}}^{a 1} / \mathrm{b}$ & -0.00363 & -0.000403 & -0.000407 & $\mathrm{~S}$ & $=0.1$ \\
\hline $\mathrm{q}_{\mathrm{v}}^{02} / \mathrm{b}$ & - & -0.000044 & -0.000046 & $\tau$ & $=60$ \\
\hline $\mathrm{q}_{\mathrm{v},}^{0^{3} / \mathrm{b}}$ & - & - & -0.000007 & & \\
\hline$q_{\phi}^{04}$ & 0.0068192 & 0.0072034 & 0.0072456 & $\alpha$ & $=0.01 \mathrm{RAD}$. \\
\hline $\mathrm{q}_{\phi}^{\mathrm{a}}$ & -0.0001112 & 0.0000129 & 0.0000303 & $\mathrm{C}$ & $=0$ \\
\hline $\mathrm{q}_{\phi}^{\circ 2}$ & - & -0.0000322 & -0.0000130 & $U$ & $=7$ \\
\hline$q_{\phi_{4}}^{0}$ & - & - & -0.0000103 & & \\
\hline
\end{tabular}

ROOT FOUND BY DETERMINANT

ITERATION WITH $\mathrm{n}=2: \quad \mathrm{p}=0.03579+\mathrm{i0.55875}$

RESULTS OF IINEAR EIGENVALUE ANALYSTS FOR $n=2,3,4$ :

\begin{tabular}{|c|c|c|c|c|c|c|}
\hline & $\begin{array}{l}n=2: \\
p=.035795\end{array}$ & $\begin{array}{r}1.55875 \\
+\end{array}$ & $\begin{array}{l}\mathrm{n}=3: \\
\mathrm{p}=.036828\end{array}$ & $\begin{array}{r}+.52903 \\
\end{array}$ & $\begin{array}{l}\mathrm{n}=4: \\
p=.036486\end{array}$ & +1.52309 \\
\hline & AMPLITUDE & 1 PHASE & AMPLITUDE & PHASE & AMPL ITUDE & PHASE \\
\hline $\bar{q}_{w} / b$ & 4.2245 & $1215.40^{\circ}$ & 4.6573 & $217.75^{\circ}$ & 4.7497 & $218.17^{\circ}$ \\
\hline & 1.2064 & $-40.51^{\circ}$ & 1.1808 & $-37.53^{\circ}$ & 1.1815 & $-37.07^{\circ}$ \\
\hline $\bar{q}_{\mathrm{w}} 2 / b$ & - & 1 & 0.03432 & $-18.25^{\circ}$ & 0.03544 & $-17.97^{\circ}$ \\
\hline $\bar{q}_{w}^{w} 3 / b$ & - & 1 & - & & 0.00530 & $-12.46^{\circ}$ \\
\hline & 0.083839 & $1-7.71^{\circ}$ & 0.87304 & $-8.79^{\circ}$ & 0.88887 & $-9.12^{\circ}$ \\
\hline & 0.05128 & $181.23^{\circ}$ & 0.05146 & $181.49^{\circ}$ & 0.050487 & $181.93^{\circ}$ \\
\hline $\bar{q}_{v_{a}}^{2} / b$ & - & 1 & 0.009128 & 169.96 & 0.009314 & $169.12^{\circ}$ \\
\hline $\bar{q}_{\mathrm{y}}^{3 / b}$ & - & 1 & - & & 0.002135 & $165.92^{\circ}$ \\
\hline$\overline{\mathrm{q}}_{\phi}^{4}$ & 1.0 & $10.0^{\circ}$ & 1.0 & $0.0^{\circ}$ & 1.0 & $0.0^{\circ}$ \\
\hline & 0.05780 & $-33.85^{\circ}$ & 0.07738 & $-30.30^{\circ}$ & 0.08213 & $-29.93^{\circ}$ \\
\hline $\bar{q}_{\phi}^{2}$ & - & $j$ & 0.02511 & $-19.79^{\circ}$ & 0.02969 & $-18.96^{\circ}$ \\
\hline $\bar{q}_{\phi}^{3}$ & - & i & - & & 0.00912 & $-22.80^{\circ}$ \\
\hline
\end{tabular}

TABLE 5.2 Modal Convergence for Convergent and Divergent Oscillatory Aeroelastic Modes (see Fig. 6-24(a)) 
ROOT FOUND BY DETERMINANT

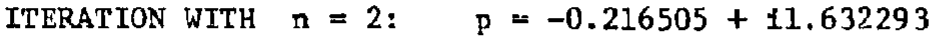

RESULTS OF LINEAR EIGENVALUE ANALYSIS FOR $n=2,3,4$

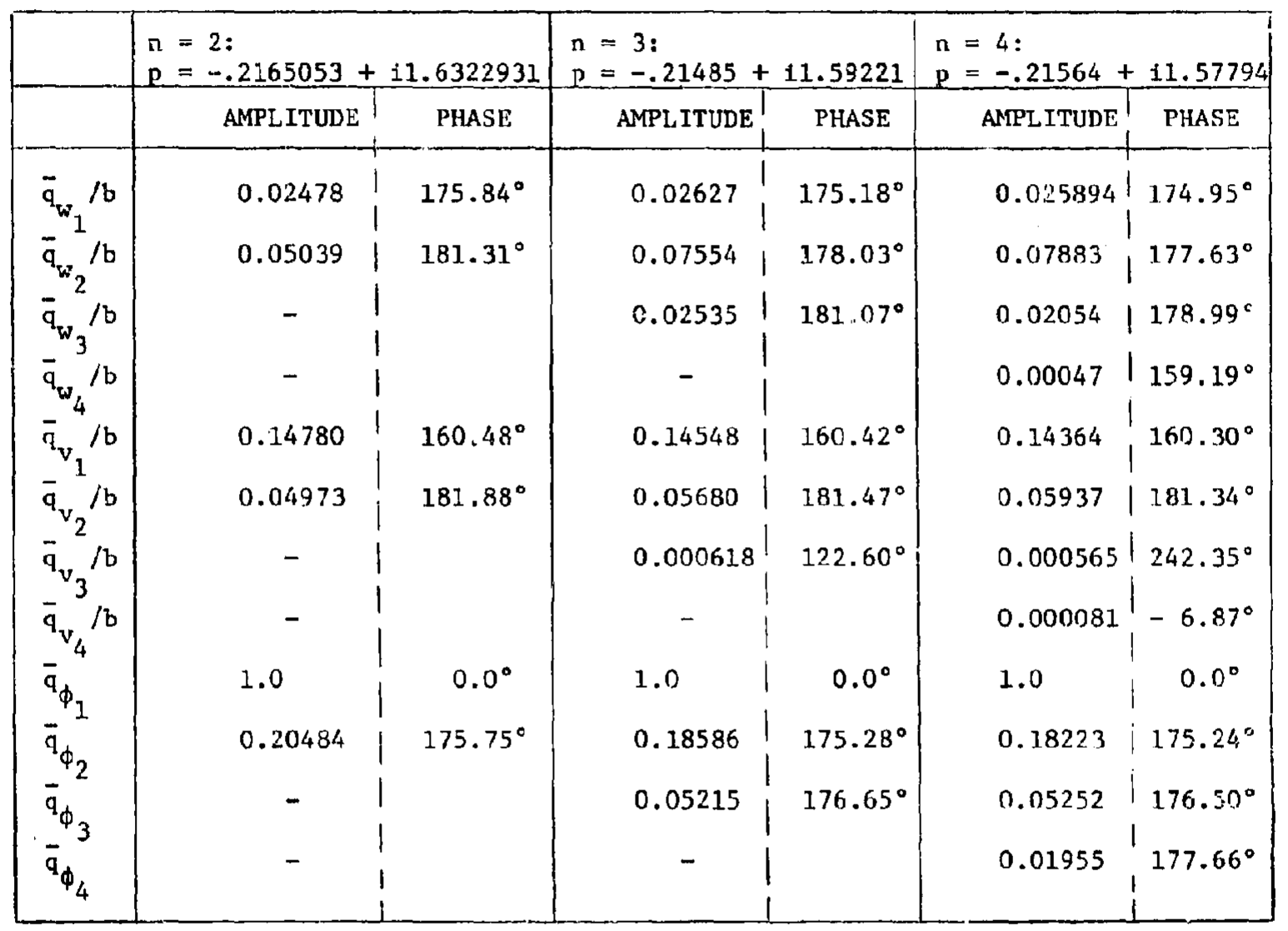

TABLE 5.2 CONCLUDEl) 


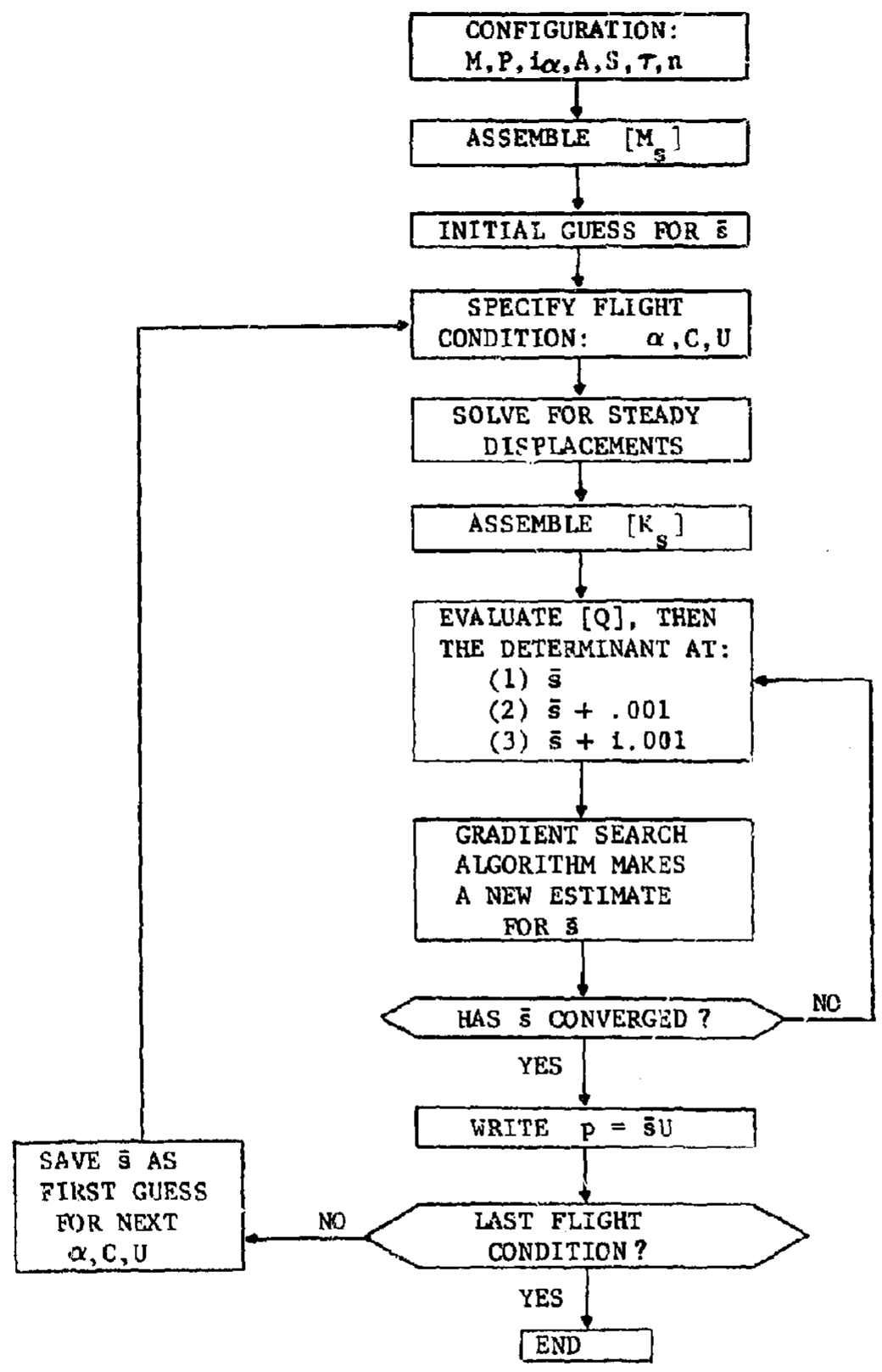

FIGURE 5-1 Flow Chart for Locating zeros of (5-8) 


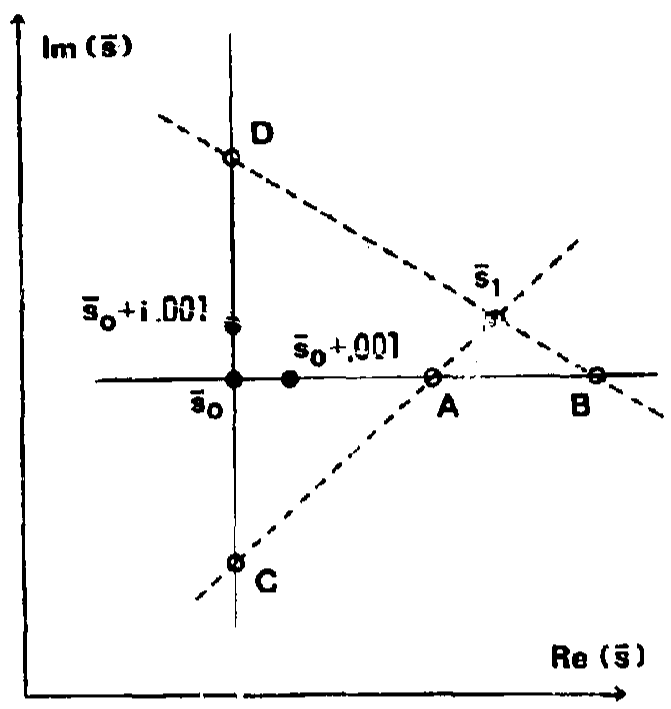

FIGURE 5-2 Gradient Search Algorithm

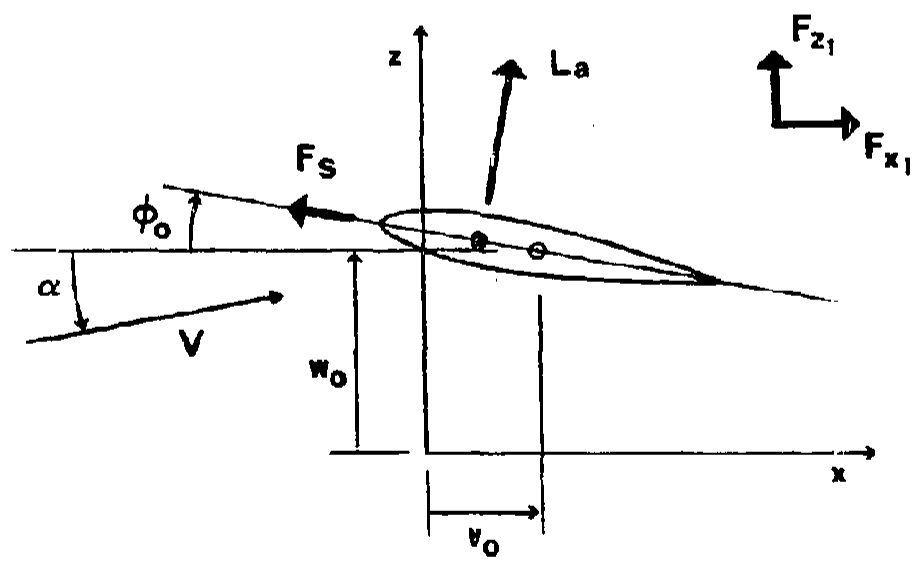

FIGURE 5-3 Resolution of Unsteady Airloacs Including Leading Edge Suction into Components $\mathrm{F}_{\mathbf{x}_{1}}, \mathrm{~F}_{\mathrm{z}_{1}}$ 
Shapter VI

AEROELASTIC MODES USING AIRLOADS FROM INCOMPRESSTBLE STRIP THEORY

\section{A. The Effect of Steady Drag on Flutter of a Nonlifting Wing}

Before considering steady-state deflections due to Ifft, a thorough understanding of the stability behavior of the cantilever wing at zero steady lift is needed. With $w_{0}$ and $\phi_{0}$ at zero, the fore-and-aft bending degree of freedom is dynamically uncoupled from vertical bending and torsion motions, and the system analyzed in Ref. 1 results. In the zero lift case, then, solutions for stabjlity involve $2 n$ aeroelastic modes consisting of coupled motions in $w_{1}$ and $\phi_{1}$; the remaining $n$ modes represent uncoupled free vibration in each of the assumed modes in $v_{1}$. As demonstrated in previous chapters, the flutter conditions obtained by this assumed mode analysis compare favorably with Ref. 1 results over all practical combinations of the parameters $M, P,{ }_{\alpha}$, $A, S$, and $C$. Owing to this good agreement, the results and conclusions of Ref. 1 apply here as well, yet the assumed mode solution method still is useful in providing additional insight into the flutter behavior of the nonlifting wing.

The parameters $M, i_{\alpha}, A$, and $S$ offer no suprising effect, and most importantly here, the nature of their influence is not altered by the inclusion of drag. The Ref. 1 results indicate that an increase in the elastic axis - A.C. offset given by $A$ is destablizing, an increase in the sectional C.G - elastic axis offset given by $S$ is destabilizing, an increase in the radius of gyration parameter $i_{\alpha}$ is stabilizing, and that the flutter speed is approximateiy proportional to the square root of the mass ratio parametex $M$. Since Ref. 1

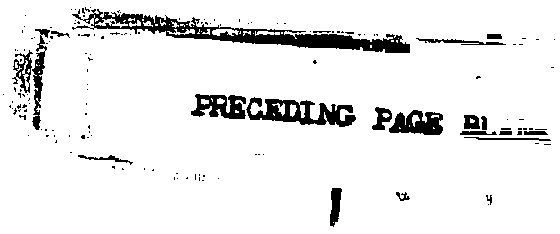


establishes that the influence of drag is not sensitive to tiese four parameters, more detailed study in this area is not considered here.

The most curious finding of Ref. I involves the effect of $c$ in conjunction with the aspect-ratio parameter $P$, which is the product of the vertical bending-to-torsion stiffness ratio with the inverse square of the geometric aspect ratio. The effect of steady drag on flutter speed is stabilizing for smaller aspect ratios (larger P) but destabilizing for larger aspect ratio wings. The reversal of the effect of drag on flutter occurs near $P=0.01$, and the behavior in this neighborhood, including flutter mode shapes, appears to be quite interesting. No conclusions regarding physical causes of this phenomenon were made in Ref. 1, however.

Figure 6-1 is a reproduction from Ref. 1, showing the effect of drag on flutter speed as a function of $P$ for intermediate values of $M, i_{\alpha}, A$, and $S$. Numbers in parentheses on the abacissa give the true aspect ratio for a typical value of the ratio $E I_{x} / G I_{d}=1.6$. Clearly wings of practical interest include the region within which the effect of drag on flutter appears to be most int:resting.

To help gain a better physical understanding of the behavior near $P=0.01$, flutter solutions for this same example have been foind via the simple harmonic method of Chapter IV, over the range $0.002 \leq P \leq 0.02$. Resuits appear in Fig. 6-2 that show flutter speeds and flutter mode shape amplitudes and phase relations as functions of $P$. Since a finice chordwise to vertical bending stiffness razio $\tau$ must be specified, and the effect of drag on flutter depends on $\tau$ as in Fig. 4-15 the value $\tau=50$ was used to allow adequately for the 
$\tau+\infty$ behavior 1nherent in the Ref. 1 formulation. Three assumed modes in each degree of freedom are used.

The flutter speeds in Fig. 6-2a closely match the Fig. 6-1 results, The flutter mode shapes include participation by the three generalized displacements $q_{\Phi_{1}}, q_{w_{1}}$, and $q_{w_{2}}$, with the remaining assumed modes contributing negligibly to the motions. In Fig. 6-2b the amplitude and phase of the two assumed bending, modes at flutter are shown for unit magnitude and zero phase angle of the first assumed torsion mode. It can be seen that for any $C$ the sharp drop in flutter speed that comes with decreasing $P$ is accompanied by a sudden change in the flutter mode shape. The amplitudes of the two bending modes merge, and the second bending mode undergoes a large phase shift. Further decrease in $P$ gives a gradual separation of the assumed bending mode amplitudes with the fundamental mote again becoming dominant.

To help visualize the physical appearance of these flutter mode shapes, phasor diagrams of the spanwise distribution of bending displacements, for $C=0.02$, are given in Fig. 6-3 for five values of $P$. Arrows depict the modal generalized displacements from Fig. 6-2, and the curves give the relative displacements along the span and their phase referenced to $q_{\phi_{1}}$. For both $P=0.02$ and $P=0.002$ aII stations are nearly in phase and the mode shape is dominated by the first assumed bending mode. But for the intermediate values of $P$, where the transition in phase of the second bending mode takes place. the displacements at different locations along the span can be over $90^{\circ}$ out of phase. 
The behavlor of flutcer modes in this range of $P$ offers the greatest discrepancy found between the results reported in Ref. 1 and the assumed mode solution, suggested by Fig. 2-1. For $P \leqslant 0.04$ in Ref. 1, collocation at only five spanwise statlons was used, and the mode shapes in bending and torsion were permitted to have spanwise phase differences. It was found, however, that these phase differences never exceeded a few degrees, in contrast to the results presented here. Possibly the use of only five spanwise collocation points did not allow enough freedom to represent the flutter mode shapt transition found using assumed modes. In any case good agreement between flutter speeds and frequencies is still observed for the two methods.

Figure 6-2 seems to indicate that the second assumed bending mode plays a significant role in the reversal of the effect of drag on flutter near $p=.01$, which coincides with the natural frequency of this mode crossing the flutter frequency. Interaction of actual aeroelastic modes is masked by the limitations of the solution method, however, which only gives neutrally stable solutions. In order to better understand these results, the Laplace transform approach of Chapter $V$ is used to allow tracing all of the aeroelastic modes in the complex plane for speeds from zero into the supercritical range.

Figures 6-4(a) - 6-4(h) give root locus diagrams for increasing speed at eight representative values of aspect-ratio paraneter $P$. Each locus originates for $U=0$ at one of the normal modes of free vibration of the structure, which are easily calculated in terms of the uncoupled assumed bending and torsion modes. Zero drag branches are shown in all of the figures, with loci corresponding to $c \neq 0$ added where their behavior differs significantly from that for zero drag. 
The first two figures represent stubby low-aspect-ratio wings, for which the strip-theory aerodynamic assumption is certainly inadequate. Due to the manner in which the Laplace variable $p$ is nondimensionalized, the predominantly first torsion normal mode of free vibration remains essentially fixed on the iw axis near 1.6 on a11 of these diagrams. The predominantly bending notmal modes move down the iw axis as $P$ decreases, since their natural frequencies decrease relative to the torsion frequencies. In Fig. 6-4(a) the bending branch of the aeroelastic modes leads to flutter, whereas in Fig. 6-4(b) the torston branch eventually becomes unstable. The normal mode having the third lowest natural frequency, predominantly the second bending assumed mode, occurs well up the iw axis and of these two diagrans and has negligible influence on flutter. These low-aspect-ratio cases show entirely twodegree-of-freedom behavior and closely resemble the root locus given Ey Edwards (Ref. 11) for a typlcal section in plunge-and-pitch motion in incompressible flow.

Figures 6-4(c) and 6-4(d) represent values of $p$ just above the condition where the effect of drag on flutter reverses. Although the Elutter phencmenon is still similar to that for larger $p$, the third normal mode frequency has now decreased sufficiently to appear on the diagram, and it produces a branch which does not lead to flutter for all values of drag.

According to the flutter curve of Fig. 6-2, for $P=0.01$ the cases $C=0.02$ and $C=0.04$ result in decrease of flutter speed from the zero-drag condition whereas for $c=0.01$ it is still increased. Figure 6-4(e) gives the $P=0.01$ root locus, which teveals that the 
aeroelastic mode emanating from the predominantly second bending normal mode now plays an important role. The next illustration, Fig. 6-4(f) with $\mathrm{P}=0.007$, gives this branch as becoming unstable for all $\mathrm{C}$. In the final two of these illustrations, with $P=0.005$ and 0.002 , the flutter phenomenon appears to be returning to the type of behavior seen for small-aspect-ratio wings, with the second bending contribution assuming a lesser influence. In Fig. 6-4(h) a fourth normal mode, the third bending mode, has made its appearance but does not noticeably influence flutter.

The nature of the aeroelastic modes on the various branches of the root locus diagrams can be clarified by looking at their mode shapes. In Fig. 6-5, phasor diagrams are used to show $C=0$ mode shapes for each branch of the .005 locus (Fig. 6-4(g)). At selected speeds both subcritical and supercritical, the generalized displacements are showl with the phase angle of $q_{\phi}$ taken as zero.

The dependence of drag's effect on flutter upon $P$ is also given for a second configuration having a smaller mass ratio $M=9.4$, more representative of light aircraft and sailplanes. Shown in Fig. 6-6, the basic behavior resembles the first configuration, with certain differences. For example, the reduction in fiutter speed by drag for small $P$ is moderate relative to that for the larger mass ratio; this is also seen in the tabulated Ref. 1 results. Also the transition as $P$ reduces through the 0.01 region is much less severe. Again the reversal coincides with the crossing of the second assumed mode frequency below the flutter frequency.

In conclusion, assumed mode results attribute the reversal of the effect of drag on flutter speed to the interaction of the structure's 
necond natural bending mode with the baslc cant1lever wing flutter mechanism involving the first bending and first torston modes. The exact reason that drag fncreases the flutter speed for $p$ above this apparent "resonance" condition and decreases it for sma1lir $r$ is noc evident in these results, and like many aeroelastir phenomena may not have a simple physical explanation. It does appear, however, that the drag force enhances the coupling of the second bending mode into the flutter beliavion and thereby magnifies its already present effect on Flutter speed.

Since the Ref. I tabulated results gtve a fafrly complete pirture of fluter of the nonlifting case in the presence of steady drag and are not disputed by current results, further work herein is dfrected towards the more general case involving steaty deformations due to 11ft.

\section{B. EfEect of Steady Deformations Due to Lift}

The good agreement in prediction of dynamic stability between the current analysis and the Ref. 1. collocation method for nonlifting wings with a steady drig force included surnlshes confidence that the modal scheme whit be successful for steady lifting conditions. The effect of steady deformations due to lift is incorporated into the dynamic stability analysis by the same means as the steady drag effect-- namely through coeffictents of thr stlffness matrix deteru! ned tin a separate tonlinear solution for the stendy-state deflections. Thus, the agreement Indfates that the scheme allowing small time dependent percurbations about a static deflection ts workting properly. When steady lifting deflections ar'e introduced through a nonzero root anple of artack $\alpha$, 


\begin{abstract}
the fore-and-aft bending is no longer dynamically uncoupled.
\end{abstract}
Coupling in both elastic terms (the stiffness matrix coefficients containing $w_{o}$ and $\phi_{0}$ ) and aerodynamic coupling terms (arising from unsteady aerodynamic force components in the $x$-d1rection) now appear, In the fore-and-aft bending dynamic equations. The $3 \mathrm{n}$ aeroelastic modes will consist of coupled motions in $w_{1}, \phi_{1}$, and $v_{1}$. Three basic wing configurations are seiected to illustrate the effects introduced by steady-state lifting deformations. Parameters $M, 1_{C}, A$, and $S$ are taken the same as in Fig. 6-1, and aspect ratio parameter $P$ is assigned three different values in order to consider wings of large, moderate, and low aspect ratio. For large aspect ratio, $P=0.005$ is chosen to provide a case for which steady drag decreases the flutter speed (Fig. 6-1). A mcdera:e aspect ratio example with $P=0.02$ having an increase in flutter spex: due to steady drag, and a low-aspect-ratio case, $\mathrm{P}=0.1$, are also included. For the typical bending-to-torsion stiffness ratio

$$
\frac{\mathrm{EI} x}{\mathrm{GI}}=1.6
$$

these examples correspond to actual retangular planforms having aspect ratios of $17.89,8.94$, and 4 respectively. The bending stiffness ratio $\tau$ now becomes important, and is given the nominal value 25 . The essential features of the flutter behavior encountered when steady deformations enter can be fllustrated by using these basic configurations as examples.

The flutter stability boundary for the moderate-aspect-ratio example is shown in Fig. $6-7$ for $c=0$ and $C=0.01$; the steady bending 
displacement $w_{0}$ of the wingtip is the measure of steady lift. An alternative would be to show flutter spesds as a function of $\alpha$, but this is a poor means for comparing curves having different steady drag and gives no information about the elastic steady deformations. A better way to indlcate the steady flight condition would be the total. lift force on the deformed wing nondimenstonalized, for example as $\frac{L \ell^{2}}{4 E I_{X} b}$. As can be seen in Fig. 6-8 this dimensionless total lift parameter, which depends on the steady twist $\phi_{0}(y)$, varies for constant wingtip deflection as $\mathrm{C}$ changes for points along the stabllity boundary of Fig. 6-7. This is because the drag force alters the relative $\phi_{0}$ and $w_{0}$ distributions for the same total steady lift. But. since this effect is small, and $w_{0}$ gives the best indication of the magnitude of the steady equilibrium deflections, this deflection is used to Indicate the steady IIft condition. In Fig. 6-7, the semispan of the wing is about 9 semichords, and steady deformations well exceeding the limtts of the moderate displacement beam theory are therefore shown. This demonstrates that flutter solutions can be found for arbitrarily large steady displacements and that 1 t is a matter of practical engineering fudgment to recognize when the assumptions made in the dertvation of the equations have been violated.

For $\mathrm{C}=0$ FIg. 6-7 indicates that the flutter speed reduces continuously with increasing steady lift until a maximum reduction of ibout $13 \%$ is achieveo at an excessively large deflection of if semichords. The frequency at flutter reduces monotonically with $w_{0}$; this effect is generally observed for all wing configurations. 
With steady drag included, the eltuation for the deformed wing is not as simple as $1 t$ is for the $\alpha-0$ case. As exhibited by the nonlinear steady deformations shown in Chapter IV drag cun greatly alter the deformation state associated with a given speed and root angle of attack. Even more importantly, drag signtflcently reduces divergence speeds, possibly to less than the flutter speed. This 1s revealed in the stability analysis when the nonlinear steady solution blows up before dynamic instabilities appear.

In Fig. 6-7 divergence speeds found by the linear vI daterminant (2-29) are also indicated for several values of $C$. Reaults of a dynamic stability analysis with $c=0.01$ also appear. The flutter speed for $\alpha=0$ and $c=0.01$ ts the single point on the ordinate and is greater than the divergence apeed for the same drag. During the search for a neutra-1y stable oscillating condition for the very small angle of atrack $\alpha=0.001 \mathrm{rad}$, and $C=0.01$, the nonlinear steady displacement solution was sensitive to $U$ to the nelghborhood of flutter owing to the proximfty of divergence. For specifled larger angles of attack, the $C=0.01$ flutter boundary is found without difficulty. The slight tncrease in flutter speed due to drag observed for $\alpha=0$ appears to be preserved in the presence of steady deformations. For decreasing $\alpha$, however, a point is reached as $\alpha+0$ at which the steady displacements are st111 nonzero at flutter. For smaller steady deflections divergence becomes the Instablifty encountered for increasing speed. For $\mathrm{C}=0.02$, divergence was observed for all lifting conditions with no flutter points found. 
As steady deformation increases from zero, the flutter mode shape undergoes a smooth transition, originating with the same zero Ifft mode shapes shown In Fig. 6-2 for $P=.02$. For zero drag, the amplitudes of modal generelized displacements which contribute significantly to the flutter mode are shown in Fig. 6-9, normalized to the first torsion mode amplitude. Their phase angles relative to zero phase for $q_{\phi_{1}}$ appear in FIg. 6-10. Participation by the first chordwise bending mode Increases steadily with increasing $w_{0}$, reflecting the increased strength of the elastic bending-torsion coupling. Vertica1 bending motions are increasingly dominated by the first assumed bending mode, and the contribution of the second torsion assumed mode increases significantly.

The flutter mode shapes at one steady lifting condition are presented in Fig. 6-11 in a form giving a clearer physical description of tine motion. Above the phasor diagram (which contains the information given in Fig. 6-9 and 6-10) is a sketch of the cyclic path traced in the $x-z$ plane by wing sertions at the wingtip and at midsemispan. Points where the first torsion assumed mode is at phase angles of $0^{\circ}, 90^{\circ}$, $180^{\circ}$, and $270^{\circ}$ are located. These diagrams emphasize the three-degreeof-freedom nature which flutter can have when steady deflections are present.

The low-aspect-ratio example $(P=0.1$ and $R \approx 4)$ shows only minor effects upon its flutter characteristies due to stcady deformations, as might be anticipated. As given in Fig. 6-12, even for the extreme condition $\alpha=.12$ rad. yielding a 1.7 semichord tip deflection at flutter, there is only a $0.62 \%$ reduction in flutter speed due to $11 f t$. The flutter mode shapes (Fig. 6-12) undergo little change, with a slight 
contribution by $q_{v_{1}}$ the only new feature. Owing to these unremarkable results, further work centers on the moderate-and-high-aspect-ratio examples, where the effects of steady deformations and chordwise forces are significant and interesting.

For the high-aspect-ratio example with $P=0.005$, the flutter behavior is quite different when steady deformations enter. Figure 6-13 shows the dependence of flutter speed upor the steady wingtip bending deflection for two drag cases, $C=0$ and 0.01 . The minimum flutter speed in this case is over $20 \%$ below its undeflected counterpart, but quite interestingly as $w_{0} \rightarrow 0$ the stability boundaries do not converge continuously to their respective zero lift flutter speeds but approach lower points on the ordinate.

The flutter mode shapes, Figs. 6-14, 6-15, explain this new behavior. As the steady deflection becomes small, the flutter mode becomes dominated by the first chordwise bending mode, and as $\alpha \rightarrow 0$ this type of instability approaches simple free uncoupled vibration in this degrea of freedom. For moderacely large steady deflection the flutter mode sisape closely resembles that for the medium-aspect-ratio wing, Figs. 6-9 and 6-10.

Flutter mode shapes for two steady-lift conditions are diagramed in Fig. 6-16 using the same technique as in Fig. 6-11. Relative to the $P=0.02$ example, this wing shows a greater amount of participation in fore-and-aft bending, and a greater contribution from the second vertical bending mode. This mode's contribution causes the noticeable difference In the eccentricities of the elliptical paths traced in the $x-z$ plane by different wing stations. The phase relationship among $q_{\phi_{1}}, a_{w_{1}}$, and $q_{v}$ appears similar in this and other examples. 
The most noticeable effect of steady drag again is 1 ts reduction of the divergence speed. Figure 6-17 gives divergence speeds found by the linear determinant (2-29) together with zero lift flutter speeds for Increasing steady drag for the moderate- and high-aspect-ratio cases. Divergence is clearly more important relative to flutter for the larger aspect ratio.

The effect of steady drag on the dynamic behavior (Fig. 6-13) appears to cause only slight adjustments to the zero drag flutter results for any steady deflection. The flutter mode shape amplitudes and phase angles show but small changes for the rather large drag $c=0.01$. The flutter speeds fall fust below the $C=0.01$ divergence speed.

The stability boundaries in Fig. 6-13 do not allow for structional damping and give no feel for the degree of stability at speeds near flutter. To gain a better understanding of the type of instability that has been found with steady deformations, and also define the overall aeroelastic behavior, the Laplace transform approach detailed in Chapter $\mathrm{V}$ is applfed to the large- and moderate-aspect-ratio examples. The true aeroelastic modes are then conveniently traced in the complex plane at any flight conditions using root locus diagrams.

Before showing the root-locus results, it is interesting to see how stabflity is suggested by the simple harmonic method with nonzero structural damping assumed. The $\mathrm{C}=0$ stability boundary of Fig. 6-13 is reproduced in Fig. 6-18, to which stability boundaries for three values of structural damping are added. The sizeable increase in flutter speed with $g$ hints that the instability is not of severe nature, and as $w_{0}$ becomes small the predominantly fore-and-aft bending 
motion is obviously very lightly damped. For small deflectlons with structural damping included, there is a change in the flutter mode back to the basic bending-torsion type encountered for zero steady 1ift. In spite of structural damping's stabilizing influence, there still exists the possibility of a reduction in flutter speed at high load factor Flight conditions.

Root locus diagrams depicting the dynamic stability of the moderateand large-aspect-ratio-wing examples with steady deflections appear in Figs. 6-19 and 6-20 respectively. Dashed lines in these figures show the zero steady lift loci of roots for Increasing speed, which are the same as the zero drag diagrams of Figs. 6-4(c) and 6-4(g). Solid lines trace the elastic modes for selected constant speeds as the angle of attack $\alpha$ is varied, and origknate for $\alpha=0$ at nonlifting roots corresponding to these speeds.

In addition to the normal modes of free vibration involving vertical bending and torsion, previously sein on the iw axis in Figs. 6-4, the first fore-and-aft bending mode natural frequency now also must be included. This normal mode romains an uncoupled, undamped aeroelastic mode at all speeds at $\alpha=0$, but a family of constant speed branches emanates from this root with steady lift included. Since its natural Erequency $\omega_{v_{1}}$ is a factor $\sqrt{\tau}$ larger than the first vertical bending assumed mode frequency $w_{w_{1}}$, this new normal mode lies on the iw axis approximately at a multiple $\sqrt{\tau}$ of the lowest normal mode frequency. This falls above the zero-lift flutter frequency for the moderate aspect ratio wing, but less than it in the high-aspect-ratio example. 
A general feature of FIg. $6-19$ and $6-20$ is that as steady lift Increases from zero, one group of constant-speed branches tends to stabilize and rapidly increase in frequency, whereas another lower frequency family of root paths reduces in stability and decreases in frequency. This latter group is responsible for tie stability boundaries of Figs. 6-7 and 6-13. In the high-aspect-ratio example, they originate from the lowest chordwise bending normal mode, but for the moderateaspect-zatio wing they originate on the zero-Iift torsion branch of the root locus. The constant-sperd branches which originate on the first and second vertical bendirg zero lift paths, for both examples, do not show much sensitivity to steady deflections.

To illustrate more clearly the role played by the chordwise bending upon stabilicy, the large-aspect-ratio example is modified by increasing the bending-stiffness ratio $T$ from 25 to 60 . This raises the dimensionless natural frequency of the first chordwise bending mode from 0.5216 to 0.9629 , which is greater than the zero lift bending-torsion flutter frequency of 0.8921 . The stability boundary calculated for this modieied example appears in Fig. 6-21 and the assuciated flutter modes are presented in Figs. $6-22$ and $6-23$. The root locus obtained via the Laplace transform method appears in Iig. 6-24(a) and the true stability of constant-speed branches yielding instability is better depicted in 6-24(b) using the damping ratio $\zeta$. The discontinuity in the stability boundary is only a consequence of the solution procedure of Chapter IV and covers a region where solutions that do exist could not be determined. It is due to an interaction of the predominanty second bending, stable aeroelastic mode with the 
roots that yield instabilfty. The simple harmonic solution method involves fixing the angle of attack a atd searching for the neutrally stable lifting condition whose speed matches the calculated flutter speed. In Fig. 6-21, the gap in the curve falls between points found for $\alpha=.0095$ and .01 rad. For all points on the left segment of the stability boundary, the simple harmonic solution yielded an additfonal highly damped eigenvalue whose frequency was slightly below the flutter frequency. On the right: portion its frequency was above the flutter frequency. From the rootlocus diagram (Fig. 6-24(a)) this highiy damped eigenvalue can be identified as the predominantly second vertical bending aeroelastic mode, and the discontinuous behavior in Fig. 6-21 coincides with the crossing of frequencies as the downward moving constant speed loci associated with flutter pass the stable second bending aeroelastic mode frequency. By correlazion with the section UI- $\Omega$ discussion of the effect of $P$ and $C$ on flutter, the general effect of the second bending mode appears to be destabilizing when fts frequency is just below that of flutter, and stabilizing when its frequency is fust above.

The llutter mode shapes (Fig, 6-22, 6-23) reflect an interesting transition as flutter frequency drops below the second bending aeroelastic mode frequency for increasing $w_{o}$. To the left of the discontinuity the mode shapes resemble the zero-lift large-aspect-ratio Elutter behavior, to which they converge as $\alpha+0$. For larger $w_{0}$. though, the modes closely resemble flutter mode shapes for the $T=25$ case (Figs. 6-14, 6-15) which in turn resemble mode shapes for the moderate-aspect-ratio example. This similarity is most evident in $q_{w_{2}}$ and $q_{\phi_{2}}$ 
In Fig. 6-25 the flurter mode shapes are dlagrammed for two lifting conditions to aseist physical visualization of the motion. The larger steady deflection iliustration is similar to those given in Fig. 6-16. But the smaller steady deformation wode shape, with its large contribution by the second assumed bending mode, has the wingtip rotating in a clockwise direction about its elliptical path for a counterclockwise motion at midsemispan. In spite of the relatively small steady deflection (a deflection of 1.226 semichords at the wingtip for a semispan of roughly 19 semichords) the motion is quite three-dimensional, indicating, a significant inertial contribution to flutter in fore-and-aft bending.

The three root-locus diagrams (Figs. 6-19, 6-20, and 6-24) exhibit the basic effect which steady deflections have upon dynamic stability when incompressible strip-theory airloads are used. The basic zerosteady-lift bending-torsion flutter root together with the first foreand-aft assumed mode produce a pair of constant-speed branches, one of which rapidly stabilizes and increases in frequency while the other decreases in frequency and becomes unstable for speeds below the $a=0$ flutcer speed. These latter aeroelastic modes axe lightly damped, and generally the onset of flutter at constant speed for increasing steady deflections would not be as severe as for that encountered with increasing speed. The reduction of flutter speed with steady deformations has been noted to be greatest when the first fore-and-aft natural frequency is near to the basic bending-torsion flutter frequency.

A better comprehension of the various aeroelastic modes comes from inspecting mode shapes at both subcritical and supercritical conditions. In Fig. 6-26, mode shapes for selected points along the $u=6$ and 7 
branches in Fig. 6-19, the moderate-aspect-ratio example, are shown in phasor form. Likewise for the high-aspect-ratio wing having $\tau=60$. Fig. 6-27 presents mode shapes for certain steady deflections alcng the $u=6$ and 7 paths from Fig. 6-24. These mode shapes disclose that the branches which stabilize and inirease in frequency consist iargely of motion in the first assumed torsion mode, having a small ${ }^{q_{v}} v_{1}$ contribution locked in a characterislic phase ralationship with $q_{\phi_{1}}$ of near $180^{\circ}$. The branches producing instabilities, furthermore, have $q_{v}$ nearly in phase with $q_{\phi_{i}}$ and give the previously shown flutter mode shapes as they cross the $i \omega$ axis. Mode stapes (Fig. 6-27) for the essentially second bending aeroelastic modes show the dominance of the second assumed vertical bending mode in this branch, which remain nearly fixed in the complex plane at a frequency close to the second assumed bending mode natural frequency.

The role of the bending-stiffness ratio $\tau$ in flutter of lifting wings is next examined. Figures 6-28 and 6-29 give flutter speeds and frequencies found for the same moderate-aspect-ratio example used earlier compared with solutions for different values of $\tau$. For $\tau=12$ the dimensionless natural frequency of the first chordwise bending mode is 0.86124 , which is only slightly higher than the zero-lift flutter frequency of 0.8332 , and its stabil1ty boundary shows the most marked decrease in flutter speed as $w_{0}$ increases from zero. The other extreme, $\tau=1000$, has a dimensionless natural frequency in chordwise bending of 7.862 , yet some decrease in flutter speed still occurs. Elastic deformation in bending about the major principal axis of the airfoil cross section should be virtually suppressed and all fore-and-aft 
motion connected with vertical bending-torsion elastic coupling. Calculations are impractical for larger $\tau$ since numerical problems begin to appear, such as convergence difficulties with the nonlinear steady deflection iterative solution.

The uniform decrease in flutter frequencies as $\tau$ is decreased reflects the additional inertia from the larger fore-and-aft motions brought about by the reduced elastic stiffness in bending about the airfoil major principal axis. Decreasing flutter frequencies for increasing steady deformations likewise should be due in part to the increasing contribution of $q_{v_{1}}$, although the increase in the relative participation of $q_{w_{1}}$ is also a factor.

In Fig. 6-30 the stability boundaries shown earlier for the largeaspect-ratio example with $\tau=25$ and 60 are compared slong ith curves having $\tau=10,40$, and 200 . The appearance is complicated by the interaction of the flutter modes with the predominantly second vertical bending aeroelastic mode as discussed earlier, and by fore-andaft bending natural frequencies sufficiently low to cause convergence as $\alpha \rightarrow 0$ to speeds below the true nonlifting flutter speed.

For both $\tau=200$ and 60 the flutter frequencies descend through the range of the second vertical bending mode frequency as $w_{0}$ is increased, causing the discontinuous stability boundaries. The flutter frequencies (Fig. 6-3I) reflect the role of this second bending mode, whose dimensionless natural frequency is 0.77904 and (as already shown) a stable aeroelastic mode with approximately this frequency exists at flutter. As mentioned in discussing the $\tau=60$ results, these curves are actually continuous. but the simple harmonic solution procedure could not produce results within the gaps. 
For the $\tau:=40,25$, and 10 cases, stability boundaries converge to free vioration in the first fore-and-alt mode as $\alpha \rightarrow 0:$ thri ir frequency curves converge to the respective natural frequencies of this made. In the case $t=40$, this frequency is 0.7862 , quice close to the seconti vertical bending assumed mode natural frequency 0.77904 . This near resonance caused diffculty in precisely locating the neutrai statilicy curves for small $\omega_{0}$, as the damping became extremelv small: of course this troublo is unimportat since even small structuzal damping would raise ilutter speeds considerably here.

The stfffness in chordwise bending is thus a factor in the fluter of liftune cantilever wings. Although stability is most adversely affected for $w_{v_{l}}$ near the zero-ift flutter frequency, this effect appears over a wide range of $i$. Higher-aspect-racio-wings apparently experience a greater decrease in flutter speed with steady deformation.s, given that $w_{1}$ is sufficiently near the $\alpha=0$ flutter frequency. The mass ratio $M$ is the only parameter which can change for a specific wing, as it depends upen alticude. In Fig. 6-32 the dependerce of both flutter and divergence speeds on $M$ for the moderate-aspectratio example appears for the complefe range of mass ratios of practical interest. Divergence speeds for several values of steady drag together with zero-iff flutter speeds int $c=0$ and 0.01 are compared. In addition zero drag flutcer speeds for steady lift giving

$$
\left(\frac{w_{o}}{b}\right)_{\operatorname{Tip}}=1.3
$$

are shown for $Y=10.40$. and 100 . This same information is 
derlcted In Fig. 6-33 for the large-aspect-ratio example except wingtip deflections of 2 and 4 semichords are ised.

Divergence speeds for all drag values vary exactly as $\sqrt{M}$, as can be seen. from the divergense determinant (2-29), whore $M$ appears only as a product with the inverse square of divergence speed. The $\mathrm{C}=0$ divergence speeds for these two examples are identical, but $U_{D}$ decreases more rapidly with $C$ for the higher aspect ratio.

For mass ratiog above about 5, noniffing flutter speeds behave approximately as $\sqrt{M}$. A minimum $\ln$ flutter speed is found near $M=5$. whith a rapid asymptotic rise to infinity following a further decrease in $M$. This reflects well known results for incompressible flow (Ref. 5 , page 247). A practical application for mass ratios sufficienty small to be theoreticaliy free from flutter is the stabiltty of hydrufolis used in high-speed marine transportation. Divergence would be the type of instability encountered. This conclusion emphasizes the importance of allowing for steady drag since its effect on divergence is propor tionally the sime for any mass ratio and the decrease of divergence speed with C. can be considerable.

Divergence is more important relative to fluter tor mass ratios around 10 than for higher mass ratios; this indicates a greater likelfirond of light aircraft and sallplanes experiencing divergence. Calculation of divergence speeds for a high-performance satiplane appones as a likely application in which allownce for steady drag effects would be destrealile.

The effect of steady doformation on flutter appears insenstrfve to $M$. For equal tip deflections, $U_{F}$ reduces by about the same proportion at each of the three mass ratios in the two fisures. For the high-aspectratlo wing, these deflected tiuter speeds correspond to the aeroolastic mode connected with chordwiso bending. as discussed for $M=40$. 
The effect of changes in the remaining parameters $A, S$, and $1_{\alpha}$ has also been investigated for the steady deformed case, but no new interesting behavior was found. Their influence at zero lift, as tabulated in Ref. 1, is carried over into the deflected flutter behavior. Stablify boundaries and flutter mode shapes for increasing steady lift show the same alterations of the basic flutter phenomenon as is demonstrated by the examples used here.

To clarify the strong influence which steady chordwise loads can have upon divergence, particularly for high aspect ratios, the dependence of $U_{D}$ upon $C$ as aspect ratio parameter $P$ is varied appears in Fig. 6-34. The corresponding zero-11ft flutter speeds for this configuration (Fig. 6-1) can be compared directly. Unquestionably, for higher aspect ratios $(P \leqslant 0.01)$ drag forces typically encountered in flight ( $1 . e ., C=0.0025$ ) can cause divergence to become no less important for flight safety than primary bending-torsion flutter.

\section{Effect of Unsteady Leading-Edge Suction Forces from Two Dimensional Incompressible Flow}

Substituting the terms in (5-20) for their counterparts in the aerodynamic matrix (5-3) allows inclusion of the linearized unsteady chordwise forces arising from Incompressible potential strip theory as described in Chapter V. This effect is present only for $\alpha \neq 0$ and will grow as angle of attack is increased. The initial trend of stabllity as a increases from zero should be unchanged from the results already discussed, but at higher steady lifting conditions leading edge suction should have a noticeable infiuence. 
Figure 6-35 displays how allowance for the linearized unsteaciy propulsive force affects the destabllizing, constant speed, Increasing steady lift branches of the root locus in Fig. 6-19, which is the moderate-aspect-ratio-wing example. For this case a tip deflection of just one semichord is a very large deformation assuming a conventional bending-to-torsion stiffness ratio $E I_{x} / G I_{d}$, since the semispan would be about 9 semichords. Thus larger deflections shown are only of academic interest, since they exceed in practice the 1imits imposed by several underlying assumptions.

Figure 6-36 recasts the Fig. 6-35 information into a better form for inferring stablitty; the damping ratio of the same root branches is plotced for wingtip deflection. Stailltty is slightly increased when leading-edge suction 1 s added, but for practical deformations its effect is really not too significant. A stability characteristlc of deformed wings, also evident in the format of $\mathrm{Fig} .6-36$, is the reduced severity of flutter onset with increasing speed at constant wo for higher load factors.

Figure 6-37 gives the manner in which the root locus for the highaspect-ratio example (Figure 6-20) is altered by allowing for linearized unsteady suction forces. Shown ts the destabllizing family of constantspeed branches associaced with the first fore-and-aft bending normal mode. Again true stabiity with steady wingtip deflection is also shown (FIg . 6-38). Also added are the constant-speed branches which stabilize and increase in frequency as steady deformations increase.

Once again the unsteady suction force has a glightly stabllizing influence. Given the same ratio $E I_{x} / G I_{d}$, this axample has twice the 
span in semichords of that for the moderate-aspect-ratio example, hence lasger wingtip deflections in semichords can be tolerated. After allowing for this factor, the unsteady chordwise potential forces do not appear to have any greacer influence on stablifty for the larger aspect ratio.

Figure 6-39 shows how stability of the modifled high-aspect-ratio example (having $\tau=60$, presented in Fig. 6-24) depends on steady deformations and on the Iinearized unsteady propulsive force. Comparison with Fig, 6-38 shows a similar effect due to leading-edge suction, but steady deformations definitely have a stronger and more immediate destabilizing effect for the stfffer chordwise bending case. This Is due to the proximity of the fore-and-aft bending normal mode frequency to the zero-1ift bending-torsion flutter frequency.

Inclusion of the linearized unsteady leading-edge suction terms derived in Section $D$ of Chapter $V$ is thus found to be stabilizing for deformed wings. Aeroelastic modes involving a primary contribution by the first chorawise bending mode appear to be most affected by these terms. With their inclision, potential flow strip theory has been fuldy exploited for this problem, and further improvement in the aerodynamics involves the compressible three-dimensional loads of Chapter VII.

\section{Two Practical Examples}

The manner in which chordwise forces and steady deformations influence the aeroelastic stability of cantilever wings has thus far been 1 ilustrated by means of idealized examples. After one has identifled the fundamental effects, it is instructive to appiy the same 
solution techniques to typlcal designs. Sallplanes, having large aspect rat1os, low mass ratio, and low operating speeds (which pewit the use of inconpressible aerodynamics), are a logical choice for practical examples. Accordingly, two existing sallplanes are modeled within the approximations imposed by the assumption of uniform spanwise mass and stiffness properties and strip theory airloads. Their stability is studied using the same techniques applied before.

The first example is modeled from information given about the Slingsby Dart $17 R$ in Ref. 15, as summarized in Table 6.1. The approach taken in determining the wing stiffnesses, in lieu of looking at wing construction details, was to use photographs in Ref. 15, from which tip deflection and twist at a load factor of 4 could be measured. The stiffness $E I_{x}$ so obtatned is clearly larger than would be expected. for the weight and type of construction; this inaccuracy is due to the uniform stiffness restriction, the rectangular planform, and strip theory steady airloads. The torsion stiffness $\mathrm{GI}_{d}$ is more reasonable. The räio

$$
\frac{\mathrm{EI}_{\mathrm{x}}}{\mathrm{GI}}=4.41
$$

turns out impractically large. This number leads to a larger value of $P$ than would be expected, which causes bending natural. Erequencles to be larger relative to torsion natural frequencies than would probably occur on the actuel vehicle. It can be added that, had $G I_{d}$ been Increased to compensate for the seemingly excessive $\mathrm{EI}_{\mathbf{x}}$, then the flutter speeds would have been absurdly high. Since wing construction detalls were not avallable, typlcaj values for the parameters $A, S$, $1_{\alpha}$, and $\tau$ were simply assigned. 
A different philosophy was applied when modelilng the second vehicle, the Gemint two-place high performance sallplane detajled in Ref. 16. With construction detalls of the metal wing structure described In ikef. 16, it was possible to assign reasonably accurate values to all six of the dimensionless parameters from a diagram of the average wing cross section (Fig. 6-40). The pertinent details of modeling the Gemini are listed in Table 6-2.

One notable result is a much lower bending stiffness $E_{x}$ than for the Dart 17R. This result will compromise the static deflectionload factor relationship but should favor the dynamic modeling. The considerable differerce in bending stiffnesses between the two examples is revealed in Fig. 6-41, which shows how the true load factor (found using the respective aircraft gross weights) varies with steady vertical tip deflection at speeds near the expected flutter speeds. The poor static modeling of the Gemint should not adversely affect its flutter results, however, tip deflection ratier than load factor should be used to measure the amount of steady lift.

The mass per unit span of thi: Gemini wing was taken to be the average for the outer two-thirds of the semispan, so as better to model it dynamically. The mass ratio-- over twice that for the Dart 17R-reflects the heavier construction needed for a two-place vehicle and the smaller average semichord. This design (Ref. 16) intentionally has a higher wing loading to optimze the glide slope, with thermalling performance improved using full-span flaps. The chordwise bending stiffness was conservatively estimated yet still yielded a quite low value of $\tau=5$. The low $\mathrm{EI}_{\mathrm{x}} / \mathrm{GI}$, ratio, together $w 1$ th the very large 
aspect rat1o, result in the extremely small $P=0.00128$. This wing thus is an extreme case in the context of the sxamples studied earlier. The effect of steady drag on divergence speeds and nonifiting flutter speeds is shown in Fig. 6-42 for the Dart 17R example and in Fig. 6-43 for the Gemint. For both, the divergence speed drops below the zero lift flutter speed at drag values which can be realistically expected in flight. The excessively large $P$ of the Dart $17 R$ model moderates the drop in its divergence speed due to drag. For flutter only a slight dependence of speed on $\mathrm{C}$ is found. On the Gemini, in fact, the $c=0.02$ flutter speed is only $0.28 \%$ less that for $c=0$. Its larger $\mathbf{S}$ tends to lower the flutter speed relative to divergence. Figure 6-44 depicts the stability boundary as affected by steady wingtip deflection for the Dart 17R. The associated flutter mode shape anplitudes and phase relations appear in Figs. 6-45(a), (b). The coupling effect between the first fore-and-aft bending mode and the primary zero-lift bending-torsion flutter mechanism appears to be responsible for a decrease in flutter speed with lift, as in earlier examples. The corresponding root locus diagram (Fig. 6-46) reveals that the first chordwise bending natural frequency by coincidence happened to fall almost exactly on the zero lift flutter frequency. This phenomenon causes the pronounced drop in flutter speed for small deflections seen in Fig. 6-44. Inclusion of the unsteady propulsive force is slightly stabilizing at large deflections, as in previous cases.

A parallel analysis of the Gemini example, given by Figs. 6-47, 6-48, and 6-49, yields a very different response to steady deflections. Flutter speed decreases only slightly at representative tip displacements, and the mode shape contributions from $q_{w_{1}}, q_{w_{2}}$, and $q_{\phi_{1}}$ show 
almost no sensitivity to $w_{0}$. The chordwise bending contribution suggests the cause, made clear in the root locus diagram. The combination of a very small $P$ and small $\tau$ produces a fundamental fore-and-aft bending natural frequency which is much smaller relative to the zero lift flutter frequency than in any example yet treated. Furthermore, the second chordwise frequency drops to near the flutter frequency. This second mode (rather than the fundamental) participates in flutter of the deformed wing, yet it is not as strongly coupled elastically with the first vertical bending and torsion modes. The aeroelastic-mode branches assoclated with the first chordwise bending mode no longer play an important role in stability with steady deformations. Indeed it has degenerated into a virtually uncoupled, neutrally damped fore-andaft vibration. The linearized effect of unsteady leading-edge suction or this type of flutter is negligible.

Evidently, for extreme cases such as the Gemini model with a foreand-aft bending frequency much lower than the zero-lift bending-torsion flutter frequency, the lowest chordwise mode will not participate strongly in flutter. The low-frequency root braiches associated with it give no cause for concern. Before this calculation is accepted as a definitive flutter analysis of an existing sailplane, however, the cantilever root boundary conditions must especially be recalled. While these results do offer insight into symmetric flutter of the actual vehicle, the possibility of anti-symmetric motions involving fuselage roll and yaw Is entirely suppressed. For the Gemini in particular, there remains the likelihood that anti-symmetric vertical and fore-and-aft bending modes may couple to produce a different type of flutter-- possibly one 
with a greater sensitivity to gteady deformations. Although the results here are Insensitive to both steaiy deflections and to chordwise forces, steady drag unquestunably plays a critical part in divergence. 
DART 17R SPECIFICATIOLS (REF, 1.5):

$\begin{array}{ll}\text { SPAN } & 55.8 \mathrm{ft} . \\ \text { AREA } & 149 \mathrm{ft}^{2} \\ \text { ASPECT RATIO } & 20.9 \\ \text { GROSS WEIGHT } & 8001 \mathrm{~b} .\end{array}$

ASSUMPTIONS :

$$
\begin{array}{ll}
\text { WING WEIGHT } & 200 \mathrm{Ib} \Rightarrow m=0.1242 \frac{\mathrm{slug}}{\mathrm{ft} .} \\
\text { AVG. SEMICHORD } & 1.33 \mathrm{ft} .
\end{array}
$$

FROM REF, 15 PHOTOGRAPHS:

$$
\begin{aligned}
& \left(w_{0}\right)_{\mathrm{TIP}}=3 \mathrm{fr} . \text { at } 4 \mathrm{~g} \Rightarrow \mathrm{EI} \approx 1,500,000 \mathrm{lb} \mathrm{\textrm {ft } ^ { 2 }} \\
& \left(\phi_{0}\right)_{\mathrm{TIP}}=1.5^{\circ} \text { at } 4 \mathrm{~g} \Rightarrow \mathrm{GI} \approx 340,000 \mathrm{lb} \mathrm{ft}^{2}
\end{aligned}
$$

FROM THIS TNFORMATION ONE CAN SPECIFY

$$
\mathrm{P} \approx 0.01 \quad M \approx 9.4 \text { (sea leve1) }
$$

ASSUME THE REMAINING PARAMETERS

$$
\begin{array}{ll}
i_{\alpha}=0.25 & s=0.1 \\
A=0.1 & \tau=25 .
\end{array}
$$

EXPRESS SPEED $V$ IN FT./SEC. IN TERMS OF $U$ :

$$
U=\frac{\ell}{b} \sqrt{\frac{J_{G}}{G_{d}}} V=0.008432 V
$$

LOAD FACTOR AT SEA LEVEL:

$$
N=\frac{4 \pi \rho V^{2} b \ell}{W}\left(\alpha+\sum_{j=1}^{n} \frac{2}{\Pi} \frac{1}{(2 j-1)} q_{\phi_{j}}^{a}\right)
$$

TABLE 6.1 Modeling of the Dart 17R Wing 
GEMINI SPECIFICATIONS (REF. 16)

SPAN

$60.5 \mathrm{ft}$.

AREA

$124 \mathrm{ft}^{2}$

ASPECT RATIO

29.4

GROSS WEIGHT

$10651 \mathrm{~b}$.

TOTAL WING WEIGHT

$400 \mathrm{Ib}$.

WEIGHT OF OUTER

20 FT.

$110 \mathrm{lb} . \Rightarrow \mathrm{m}=0.1708 \frac{\mathrm{slug}}{\mathrm{ft} .}$

ASSUME $\quad b=1.00 \mathrm{ft}$.

PROPERTIES ESTIMATED FROM THE TYPICAL SECTION (FIg. 6-40)

$\begin{array}{ll}\mathrm{EI}_{\mathrm{x}} & 444,9001 \mathrm{~b}-\mathrm{ft}^{2} \\ \mathrm{GI}_{\mathrm{d}} & 402,400 \mathrm{lb}-\mathrm{ft}^{2} \\ \mathrm{EI} & 2,179,000 \mathrm{1b}-\mathrm{ft}^{2} \\ \mathrm{~J} & 0.04697 \mathrm{slug}-\mathrm{ft} . \\ \mathrm{a} & -0.4 \\ \mathrm{~s}_{\mathrm{e}} / \mathrm{m} & 0.2322 \mathrm{ft} .\end{array}$

RESULTING DTMENSIONLESS PARAMETERS
$M=22.9$
$A=0.1$
$P=0.00128$
$S=0.23$
$i_{\alpha}=0.275$
$\tau=5$

EXPRESS SPEED $V$ IN FT./SEC. IN TERMS OF $U$

$$
U=\frac{l}{b} \sqrt{\frac{J}{G I_{d}}} v=0.010335 v
$$

TABLE 6.2 Modeling of the Gemint Wing 


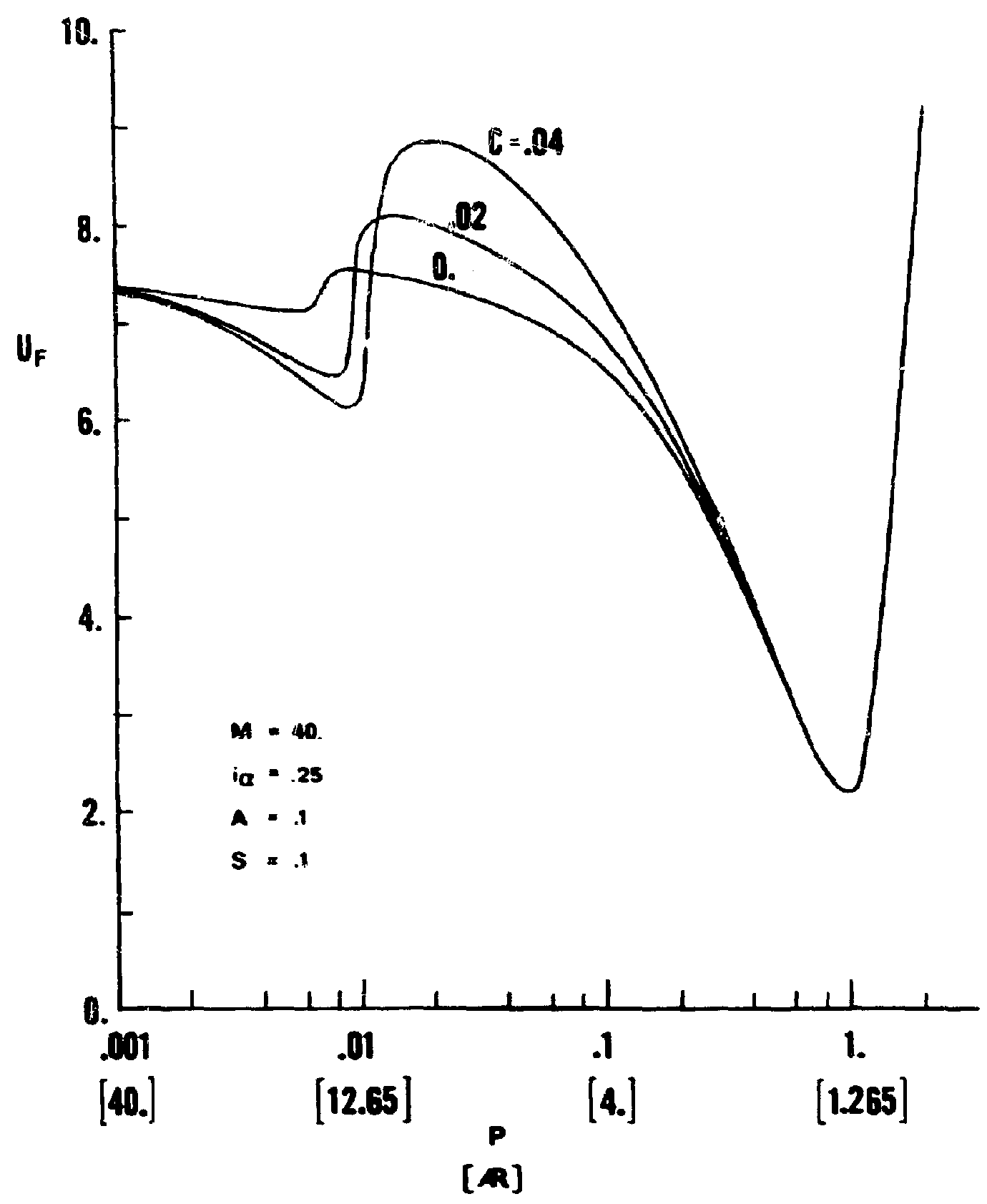

FICURE 6-1 Dependence of Flutter Speed on $C$ and $P$. Ref. I Solution 

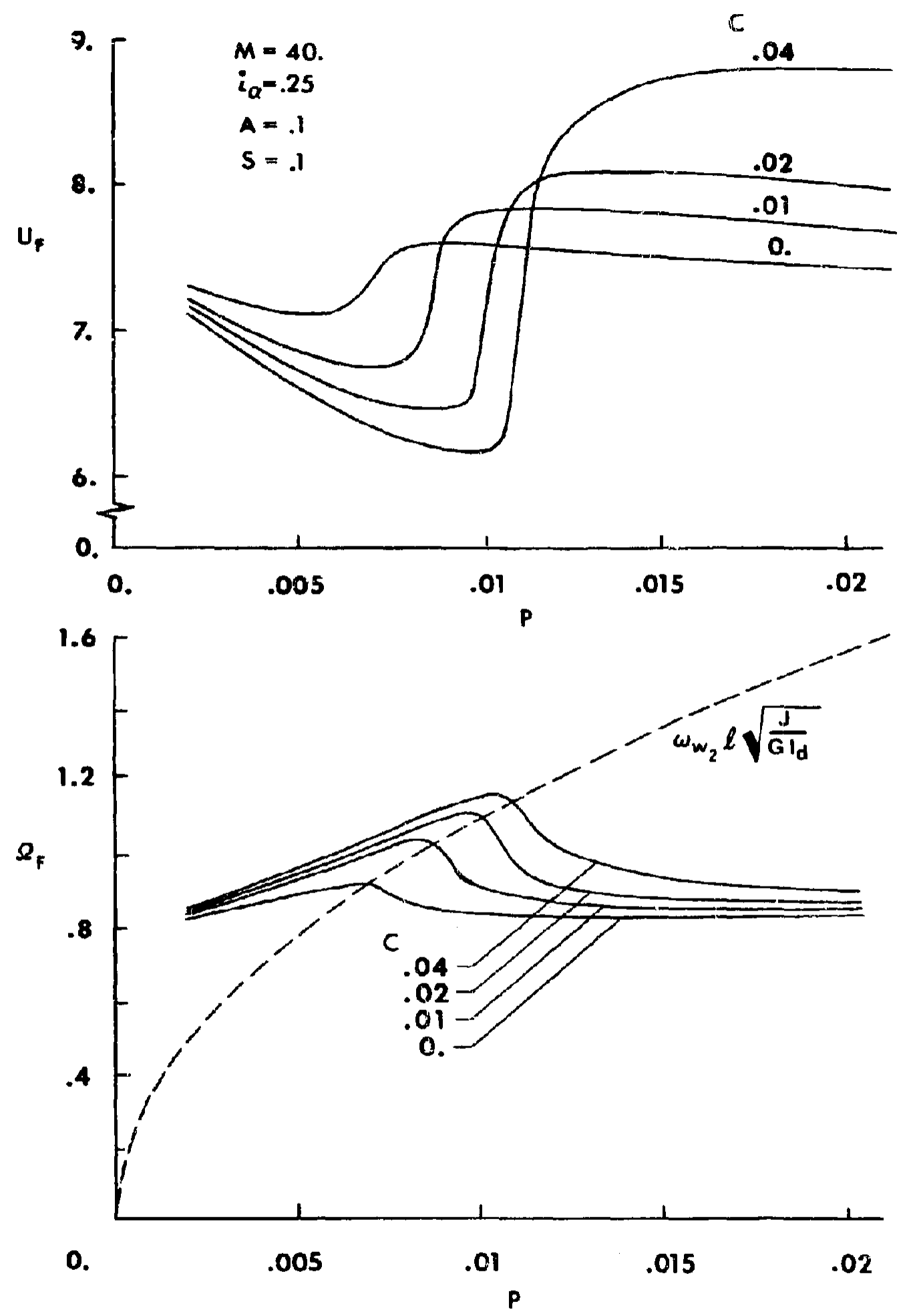

FIGURE 6-2(a) Flutter Speeds, Frequencies, and Mode Shapes for Different $C$ as $P$ Varies, Modal Solution of the Fig. 6-1 Configuration 

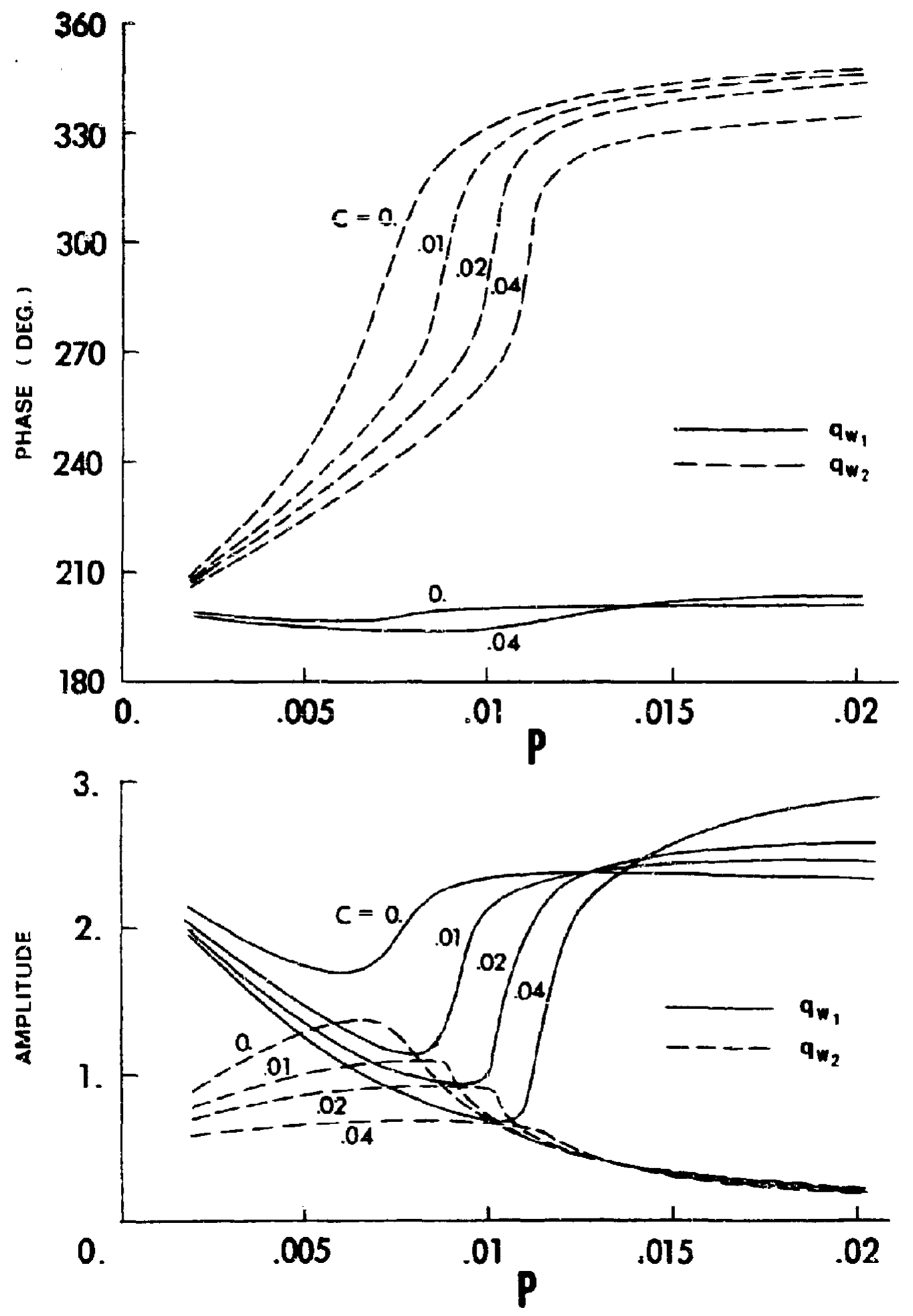

FIGURE 6-2(b) 


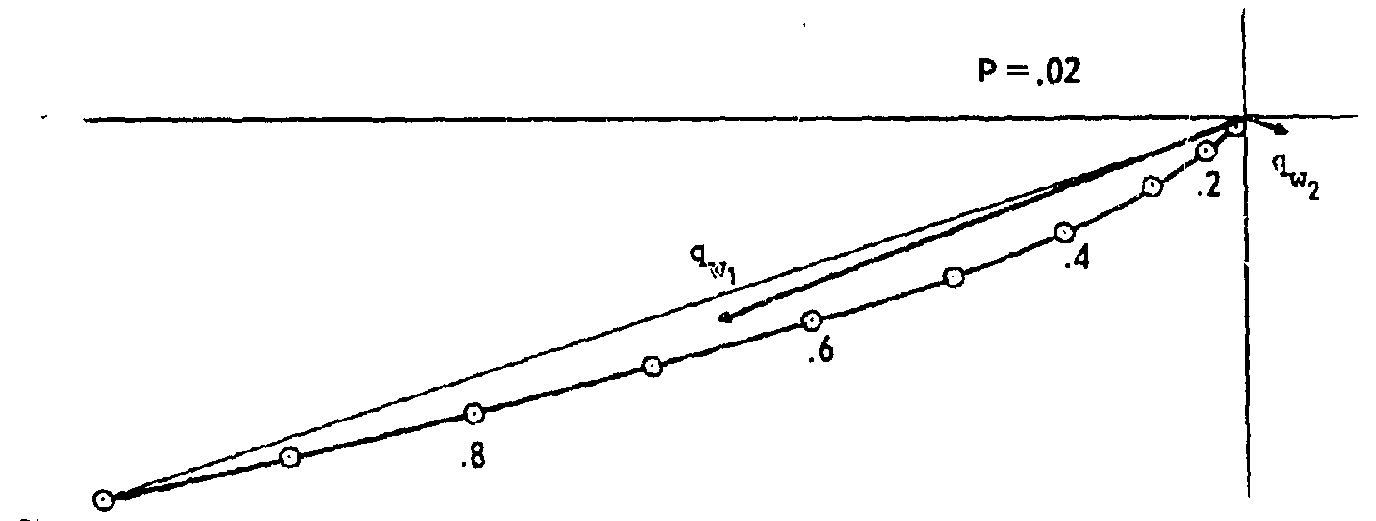

$\tilde{y}=1.0$
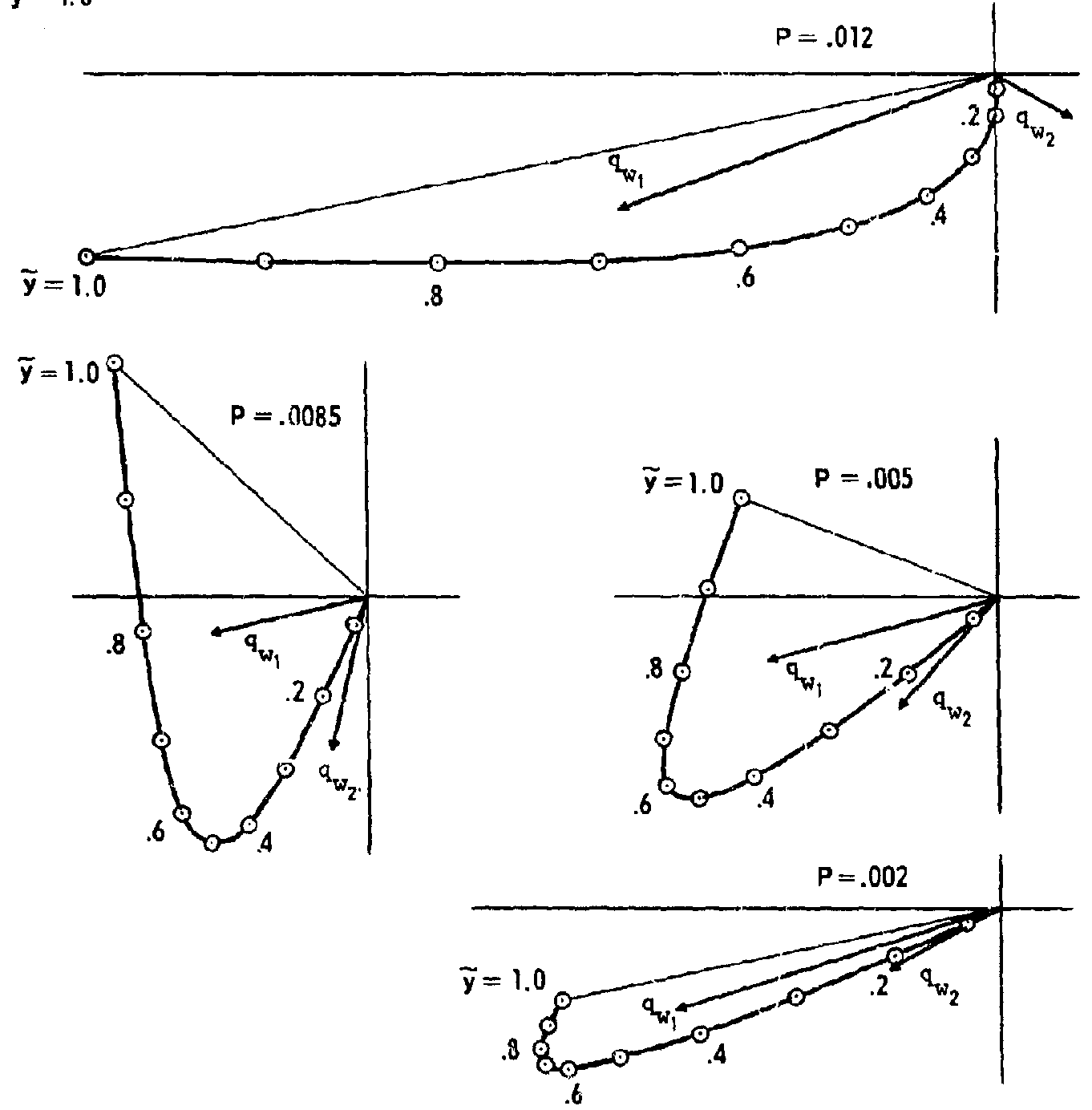

FIGURE 6-3 Phasor Representation of Flutter Mode Bending Displacements From Fig. 6-2 for $\mathrm{C}=0.02$ at Selected Values of $\mathrm{P}$ 


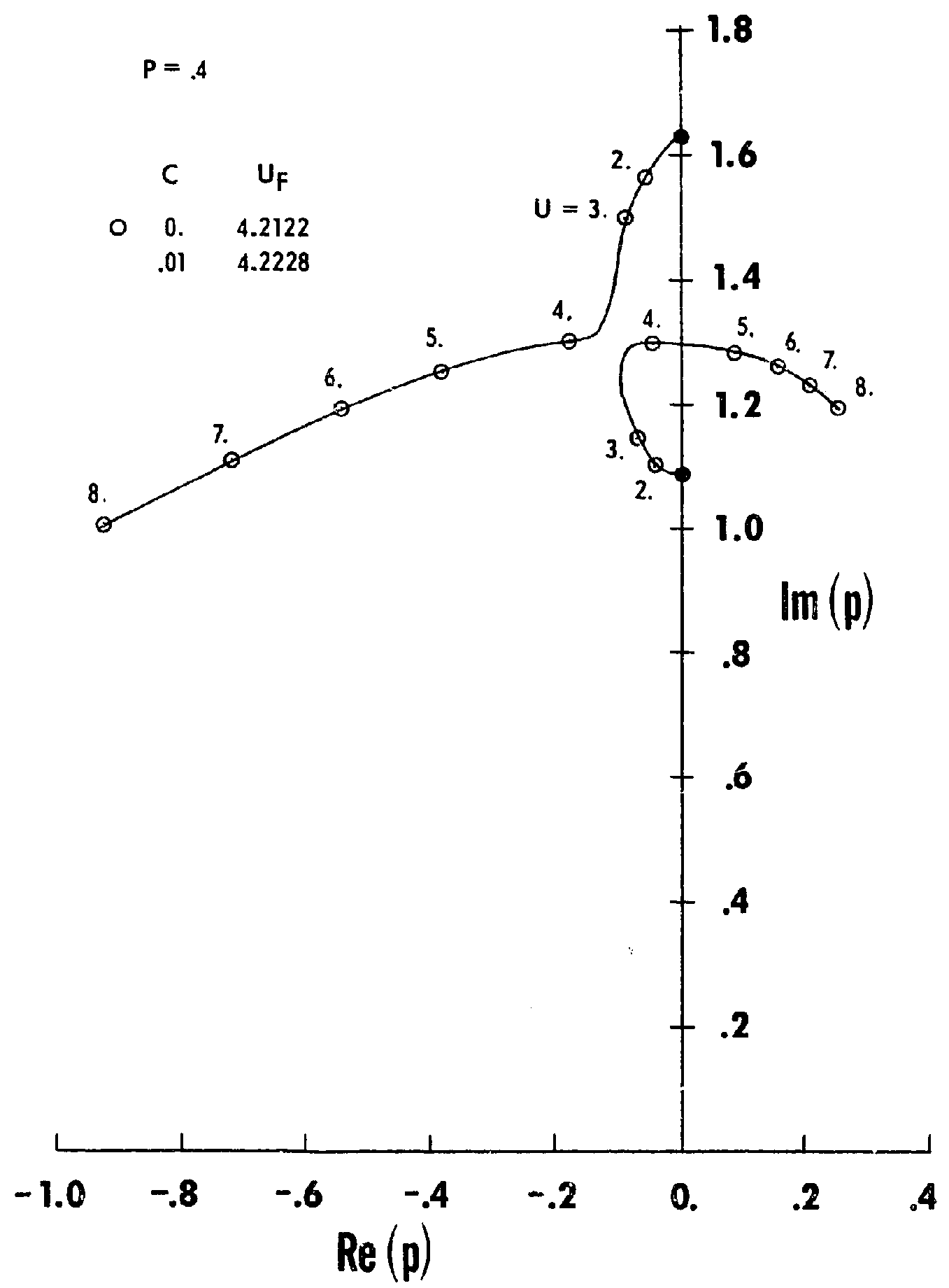

FIGURE 6-4(a) Locus of Roots as Speed Increases From Zero to Supercritical for Eight Values of $P$, with $M=40,1_{\alpha}=0.25, A=0.1$, 


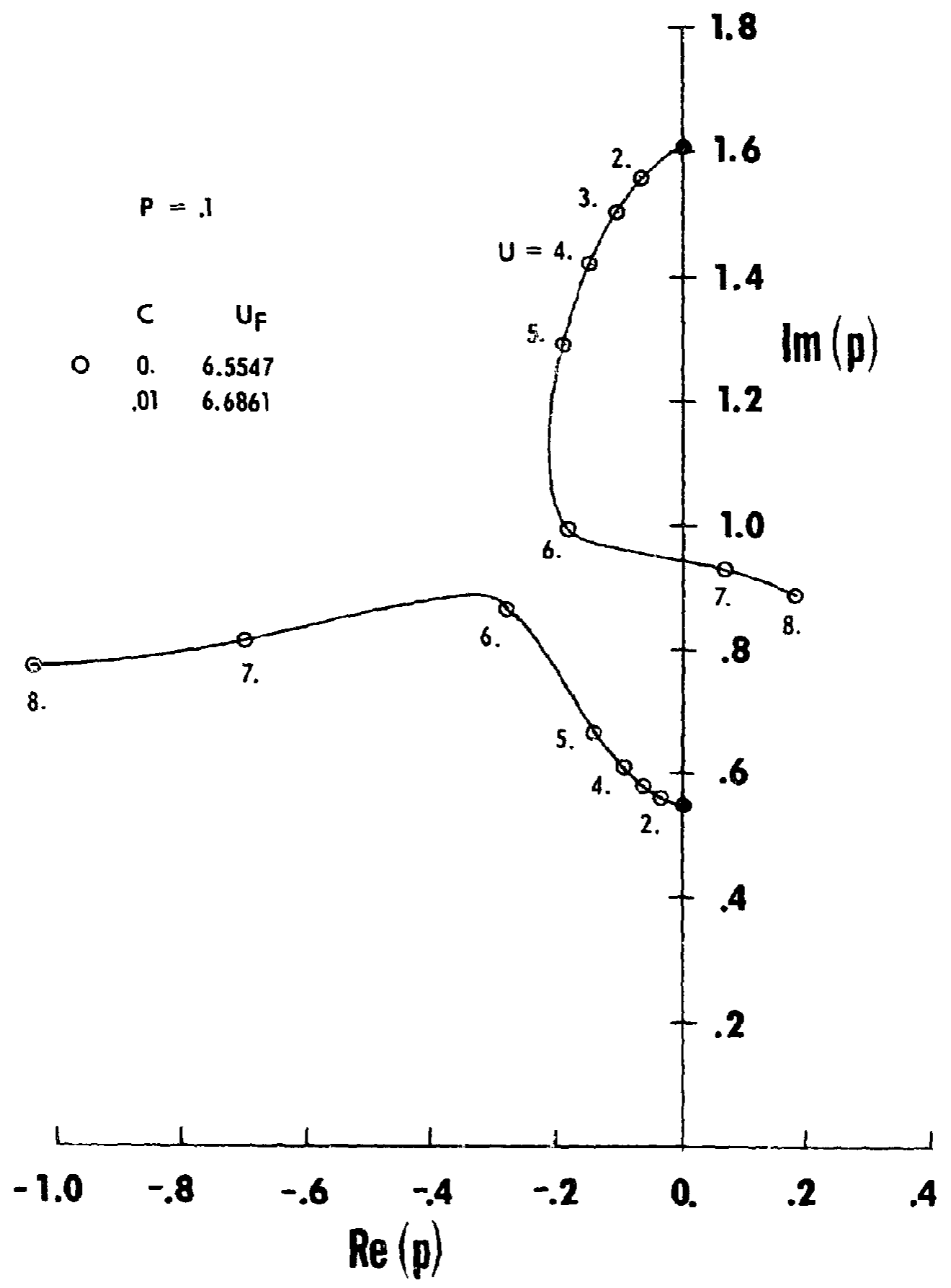

Figure 6-4(b) 


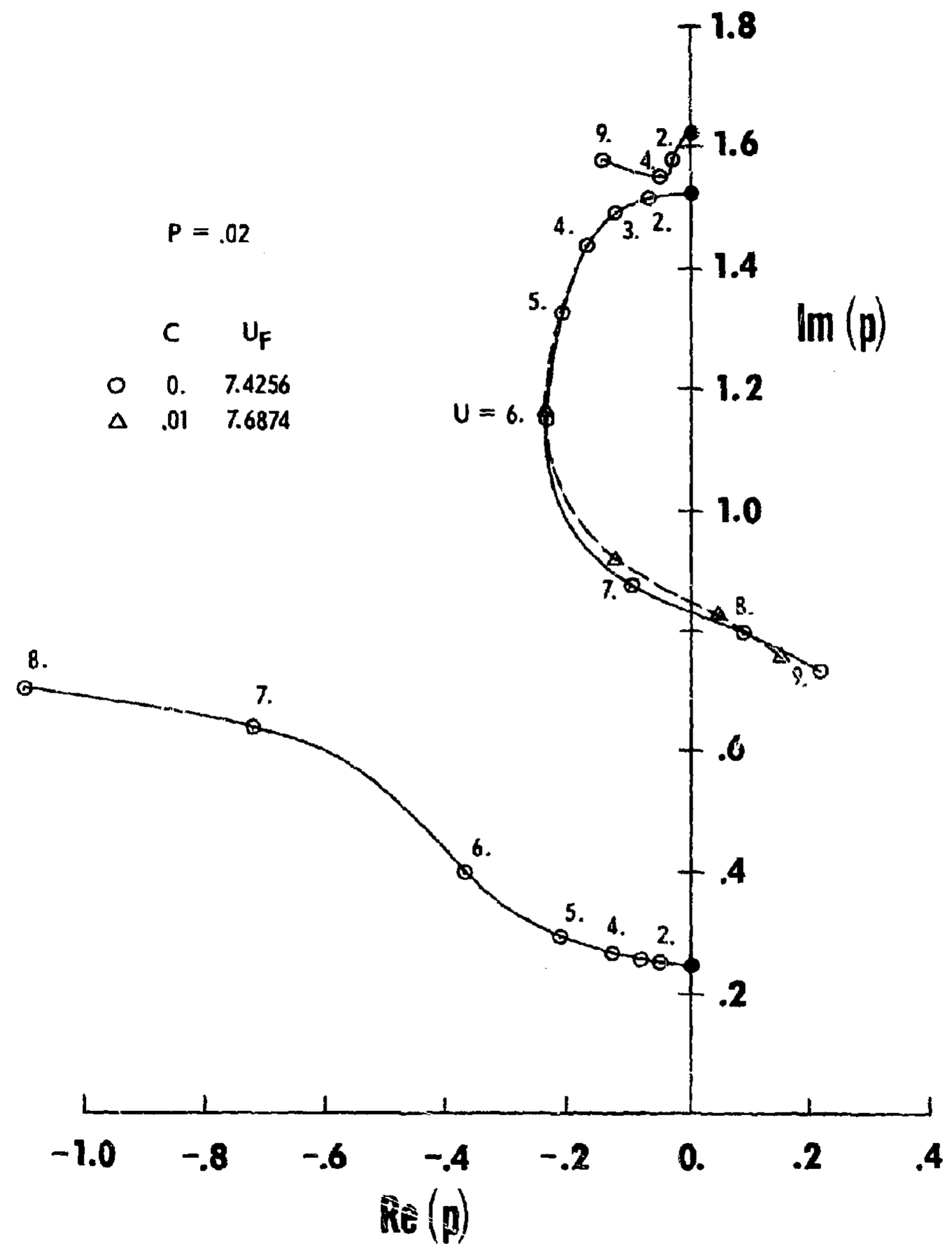

FIGURE 6-4(c) 


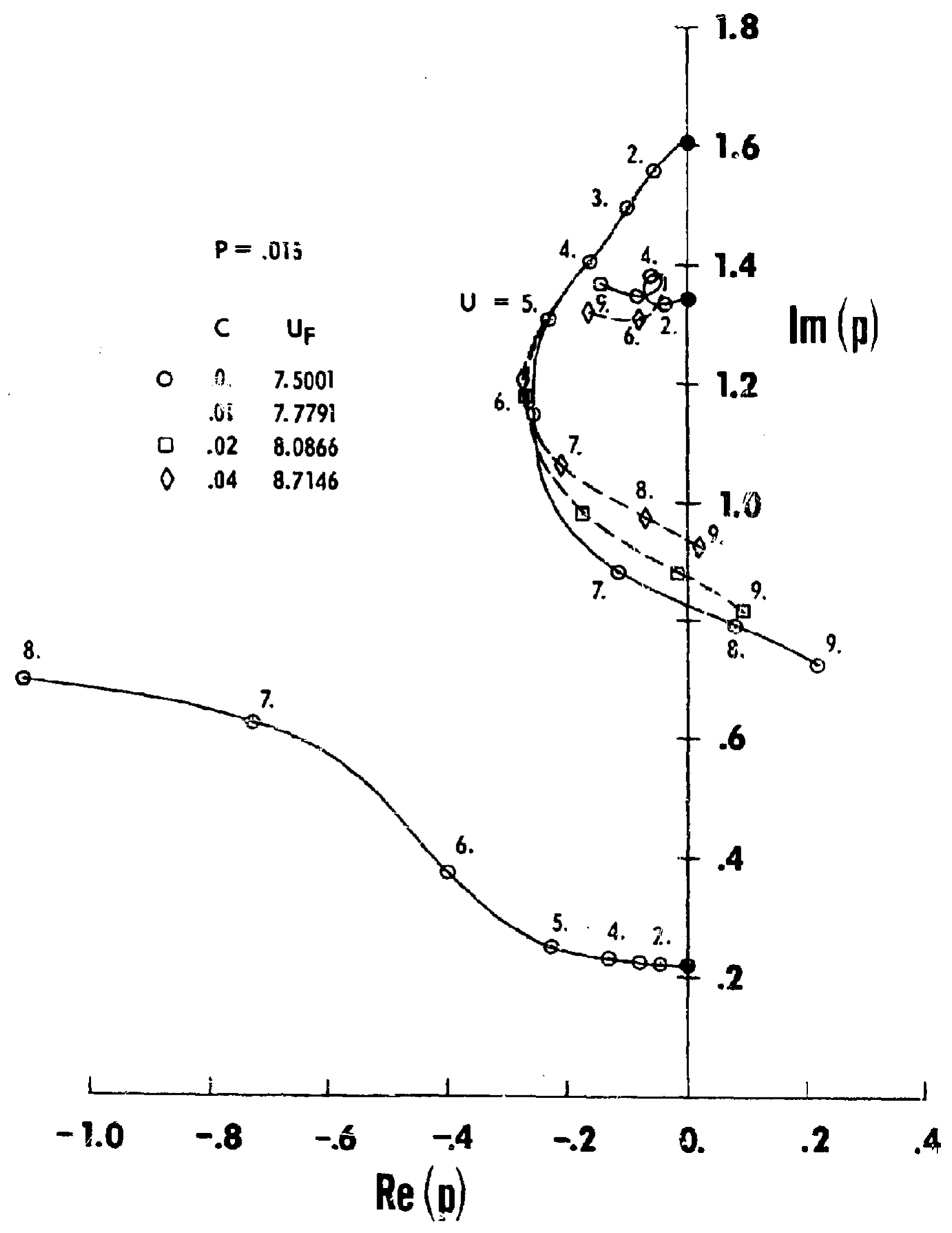

FIGURE 6-4(d) 


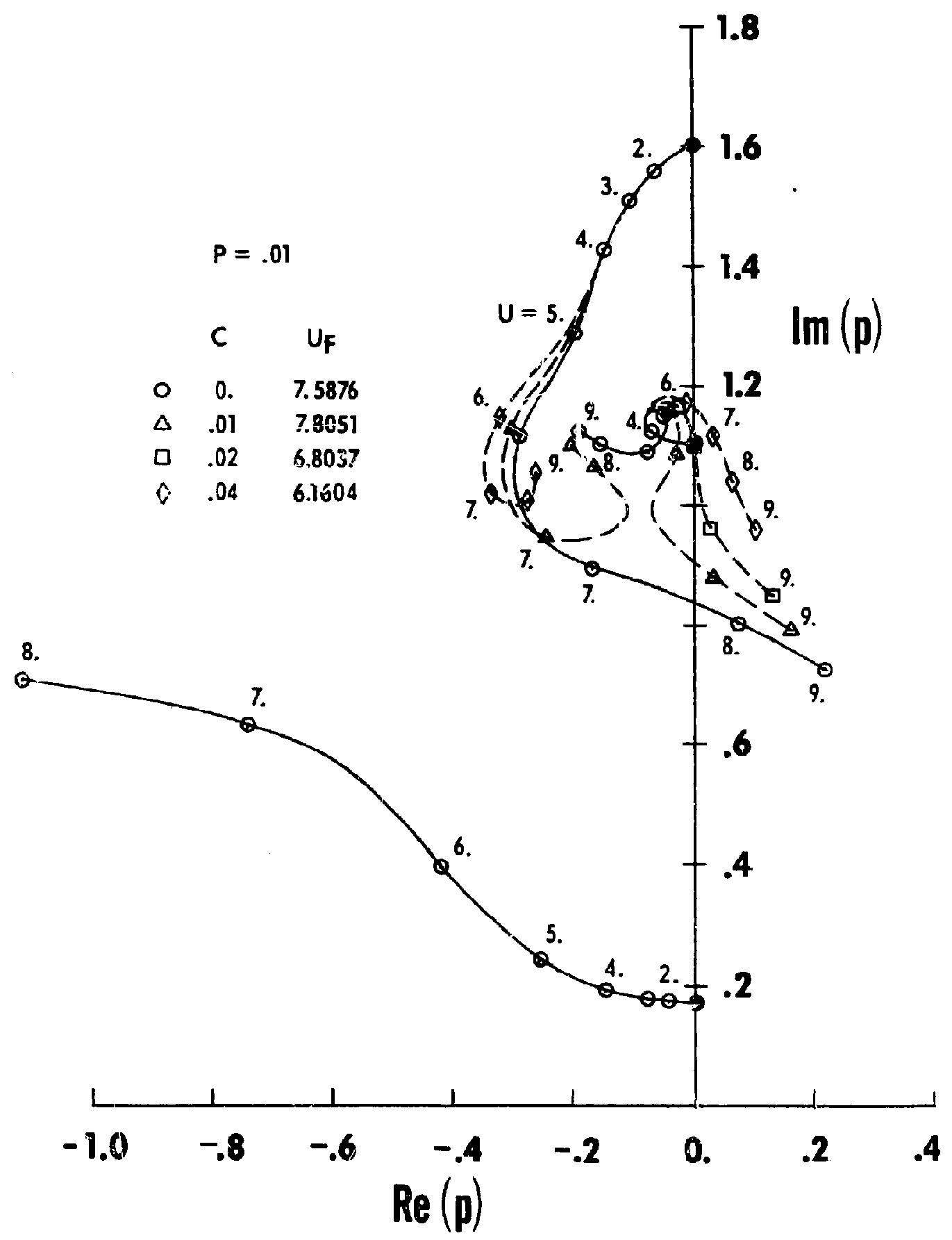

FIGIRE 6-4(e) 


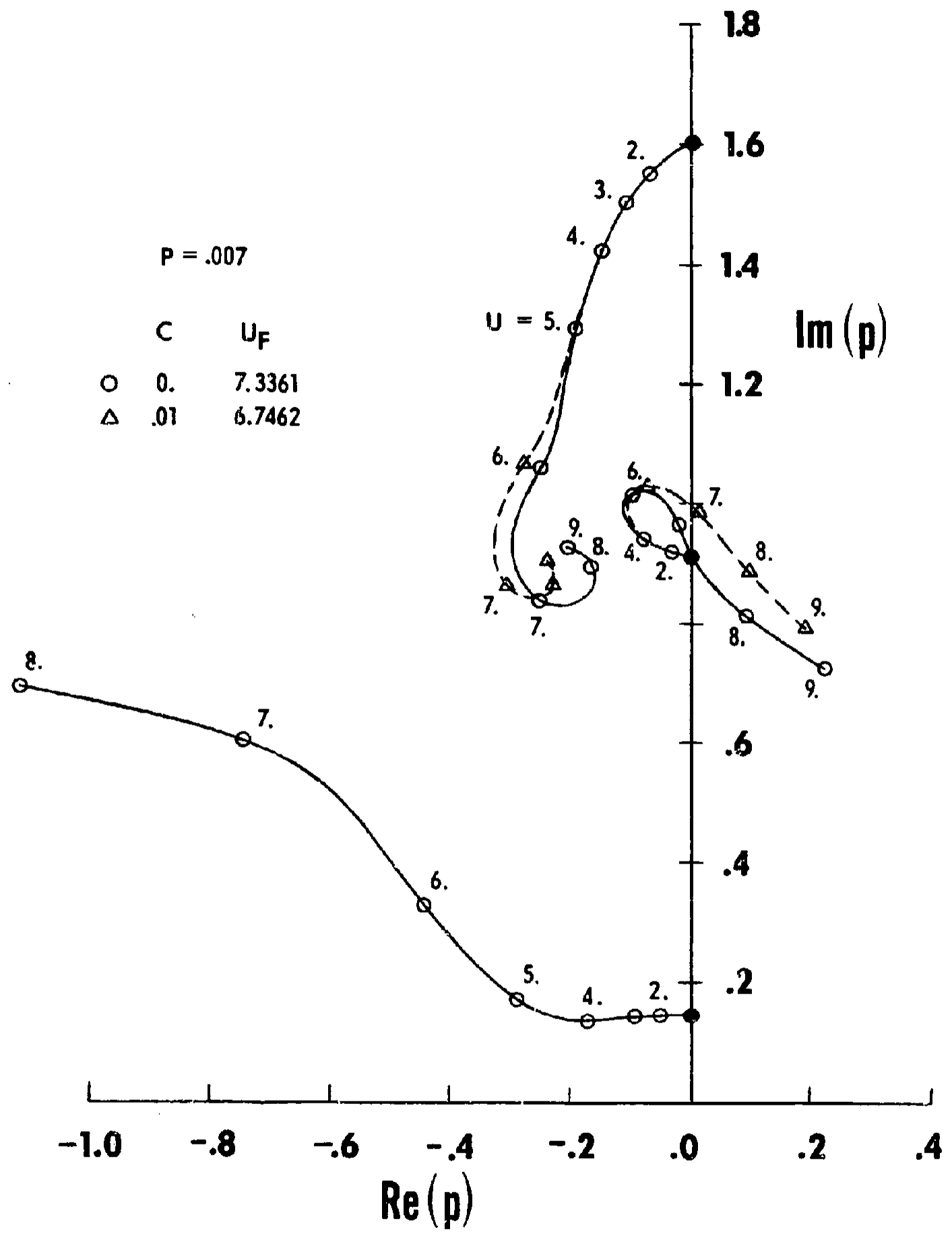

FIGURE $6-4$ (f) 


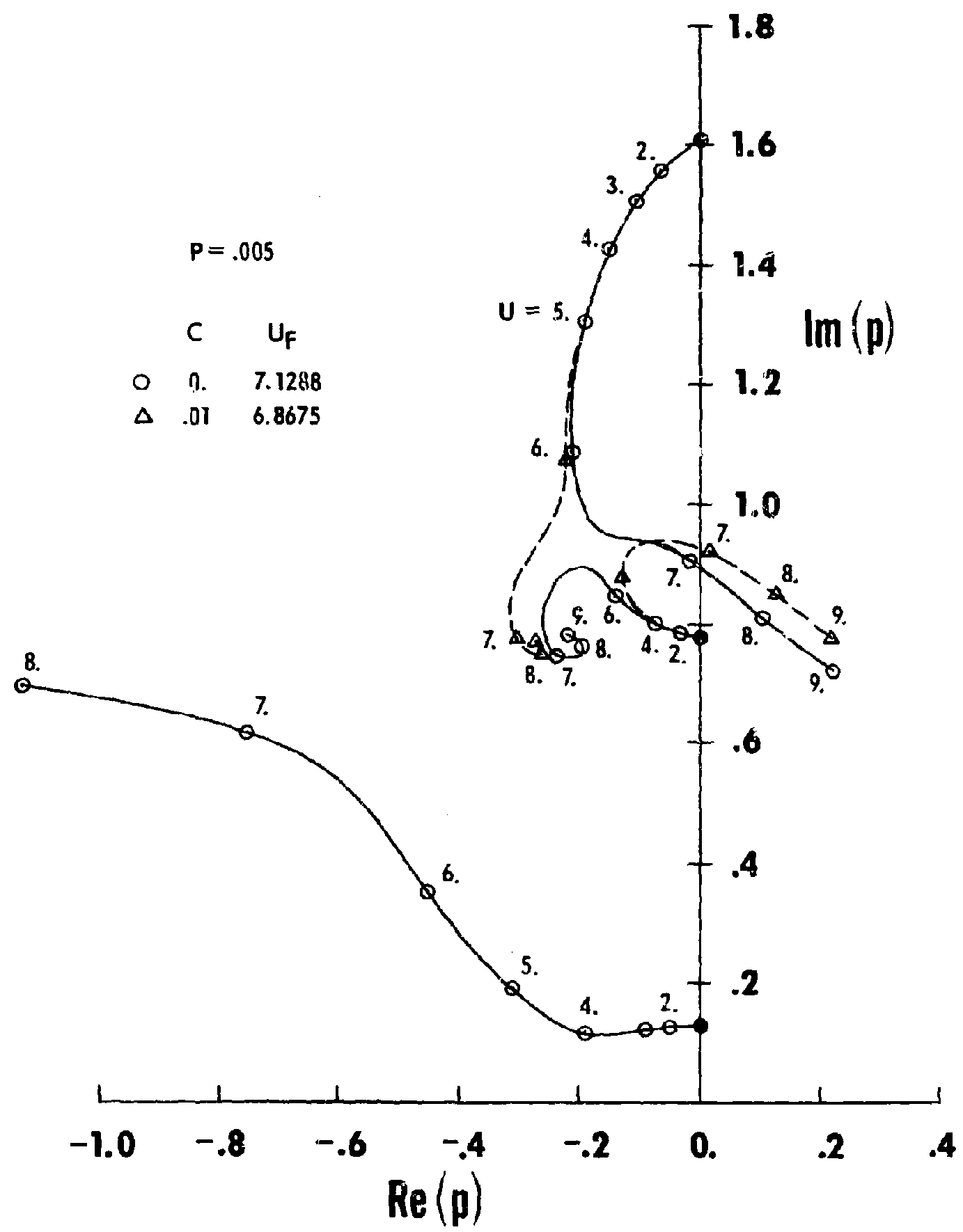

FIGURE 6-4(g) 


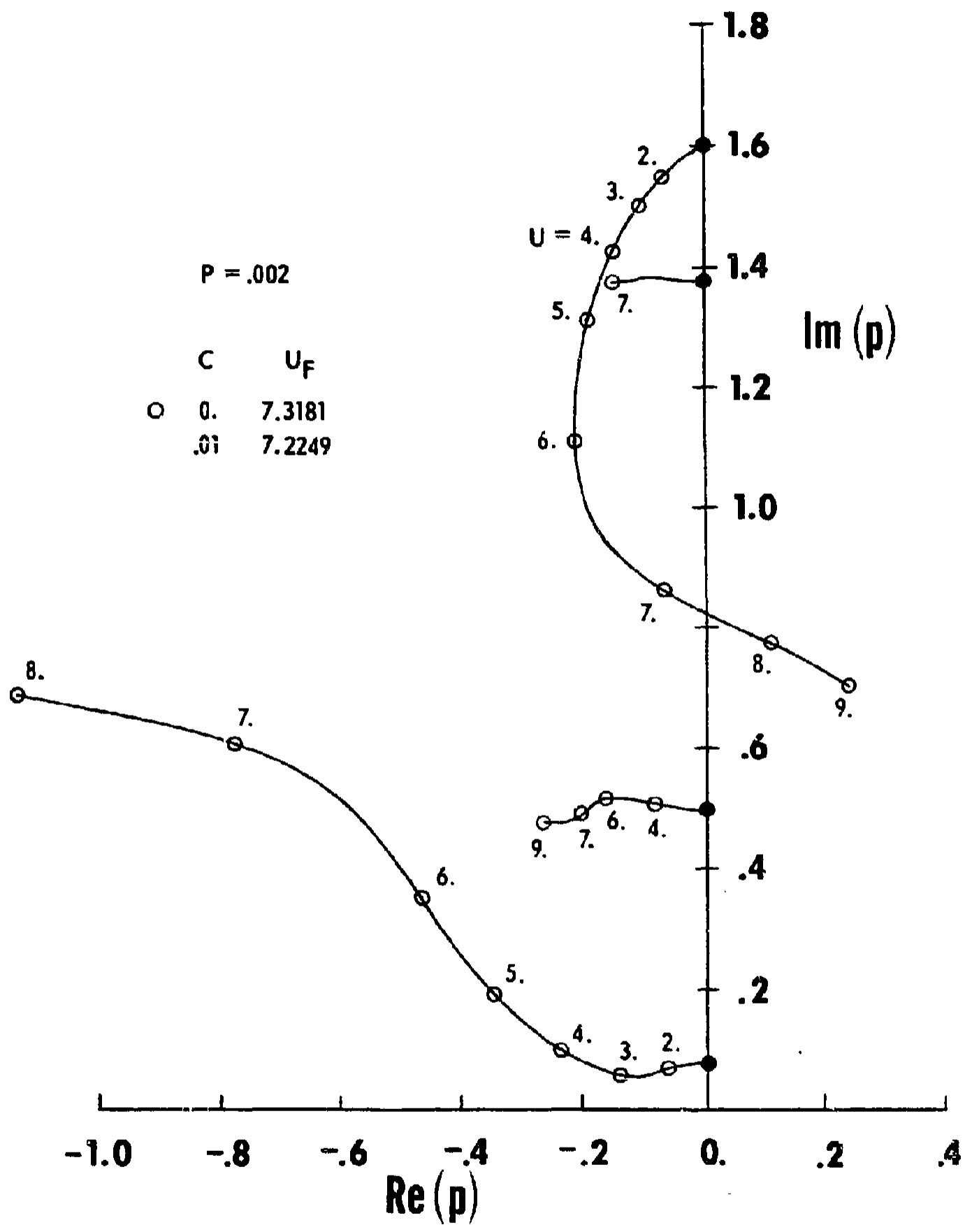

FIGURE 6-4(h) 

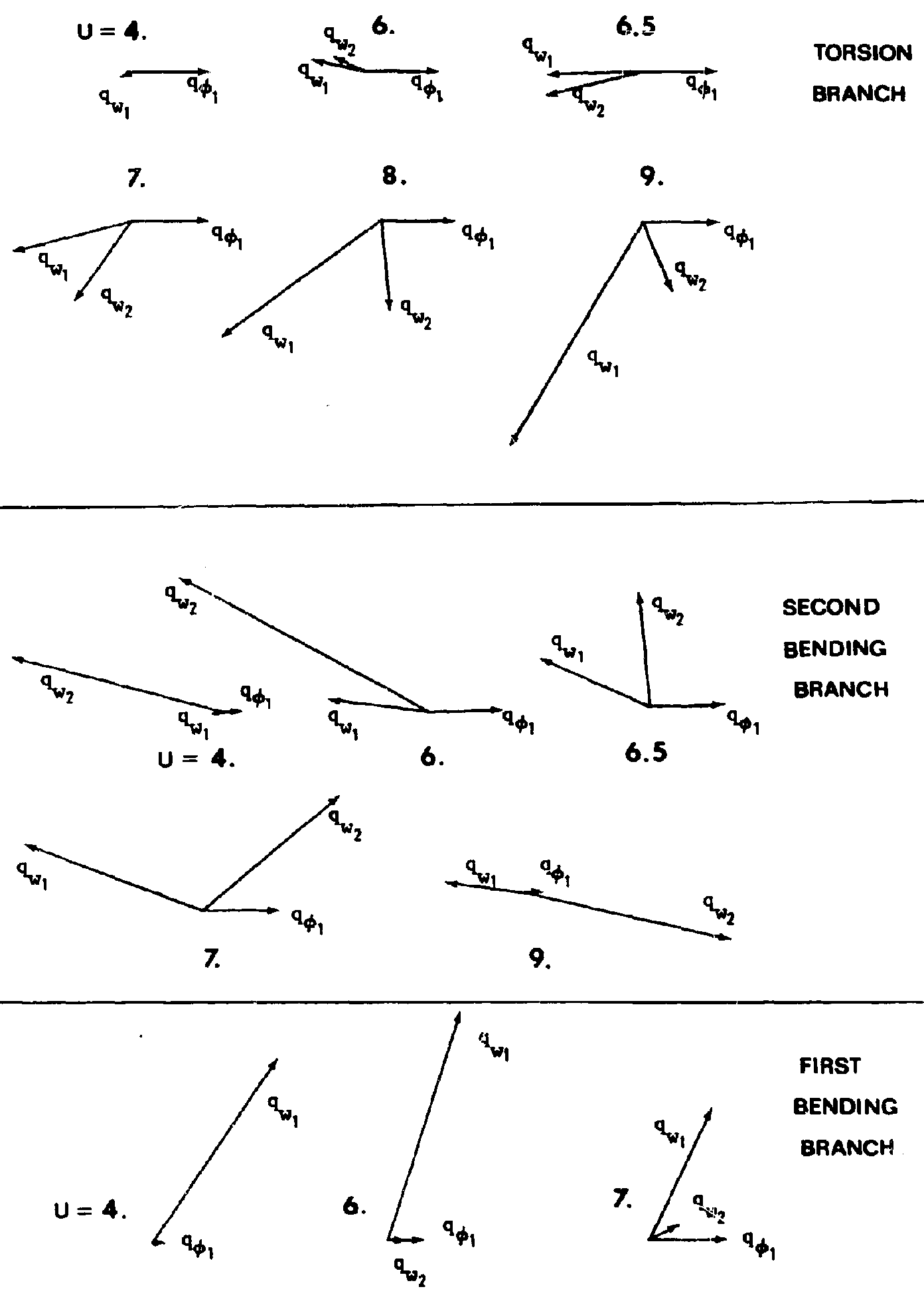

FIGURE 6-5 Mode Shapes for Selected Roots on Each Branch of Fig. 6-4(g) for $C=0$ 

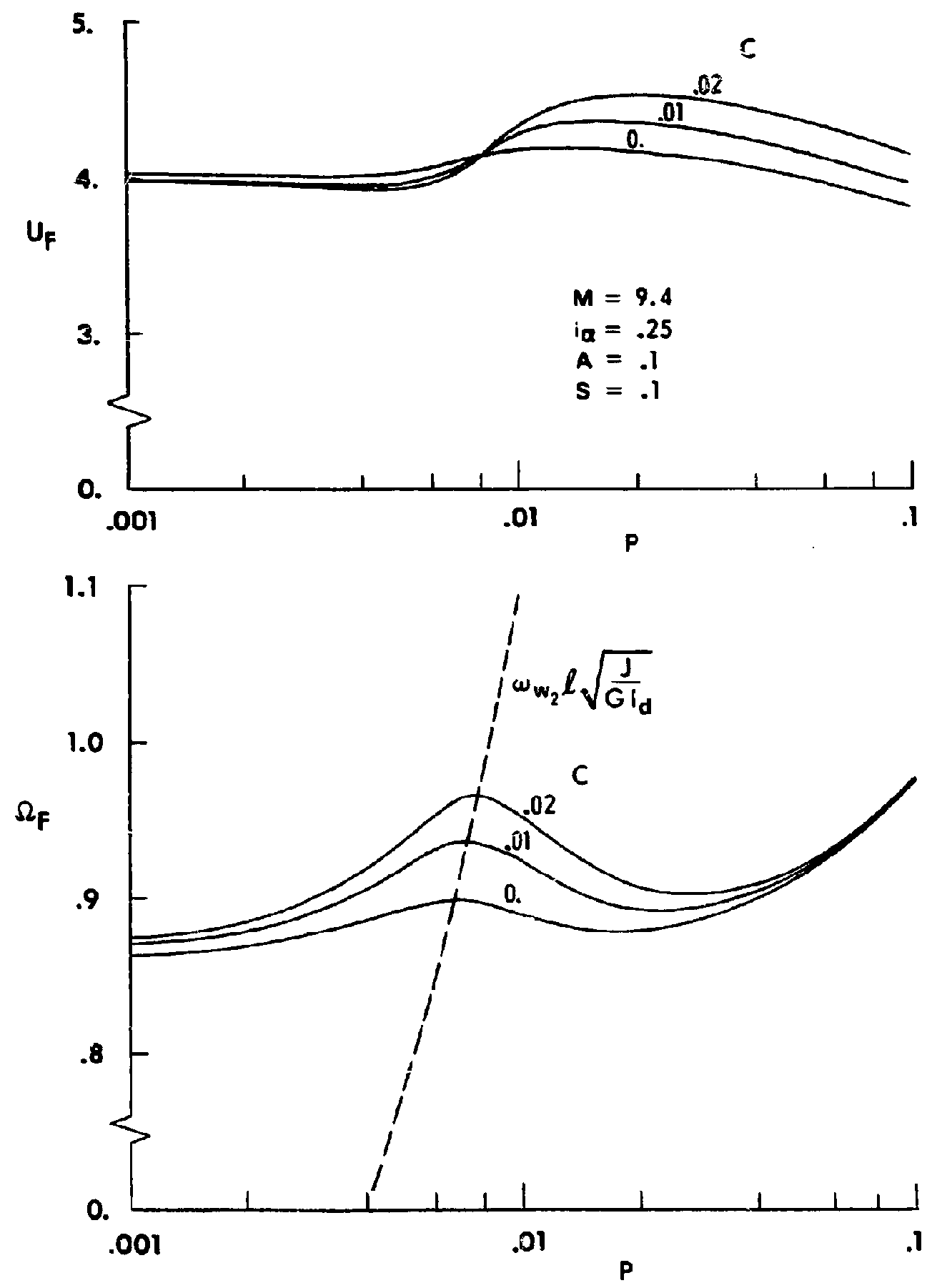

FIGURE 6-6(a) Flutter Speeds, Frequencles, and Mode Shapes for Different $C$ as $P$ Varies, Fig. 6-2 Configuration with Smalier Mass Ratio 

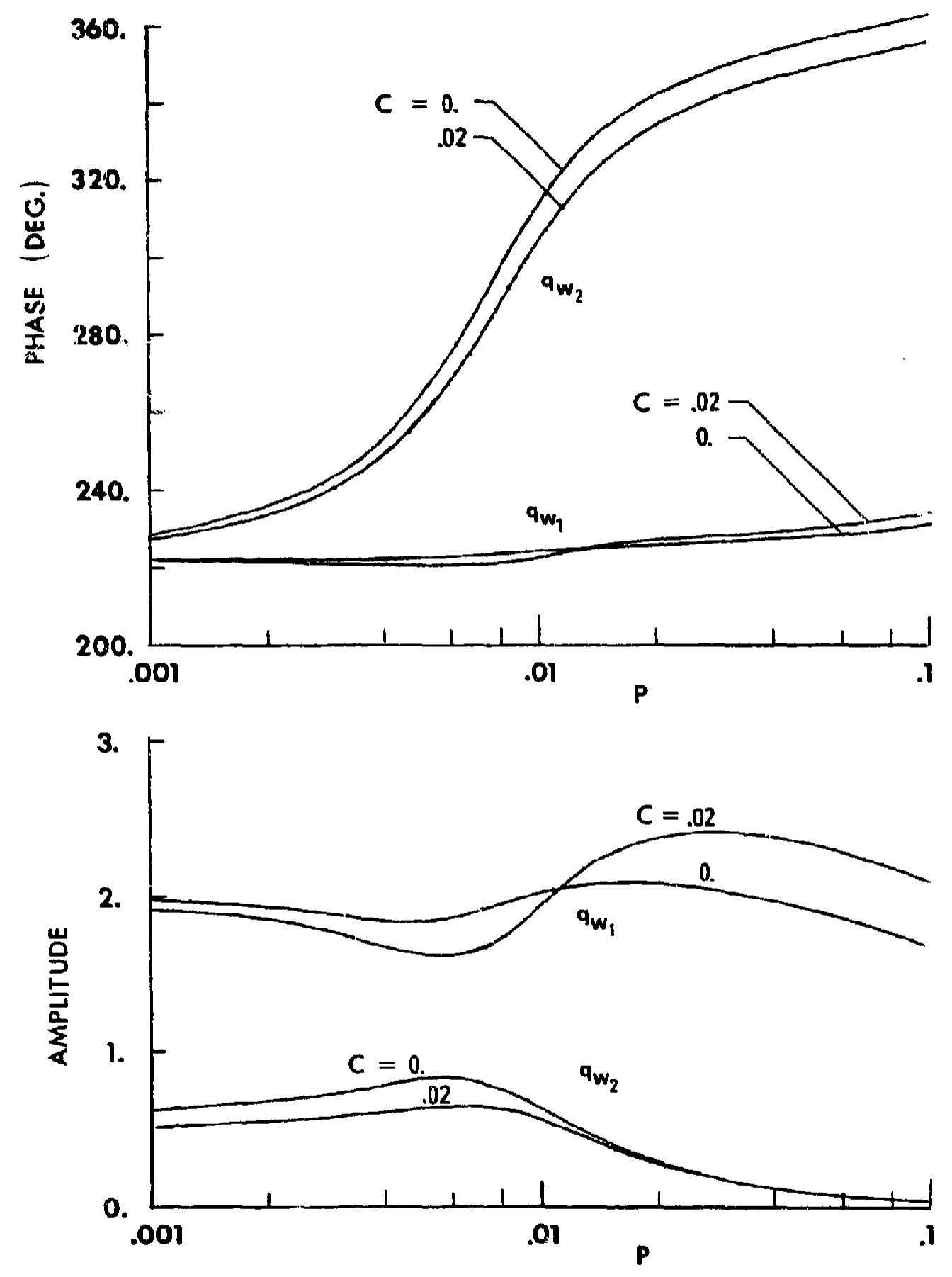

FIGURE 6-6(b) 

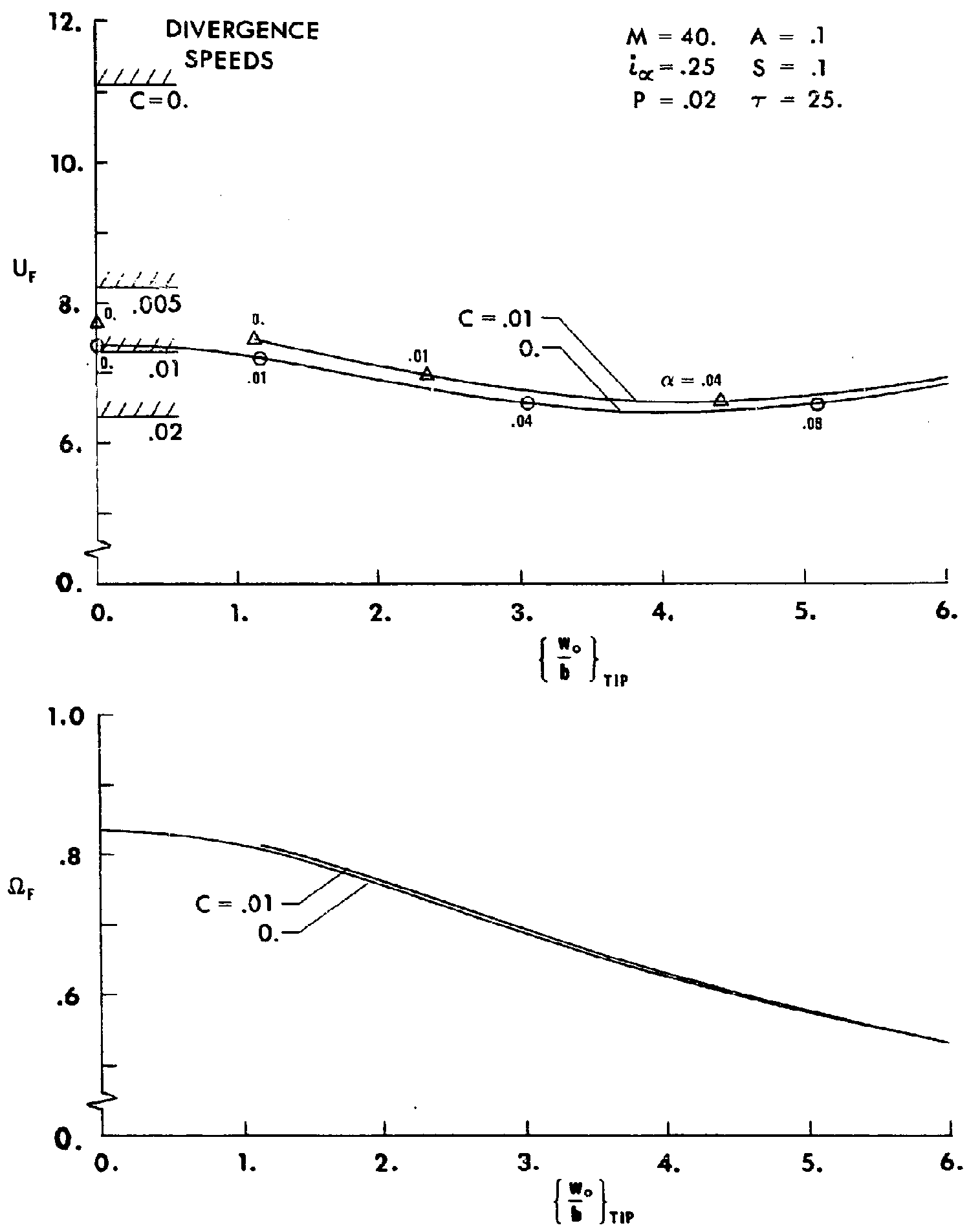

FIGURE 6-7 Flutter Speeds and Frequencies as Affected by Steady Deformations: Moderate-Aspect-Ratio Example 


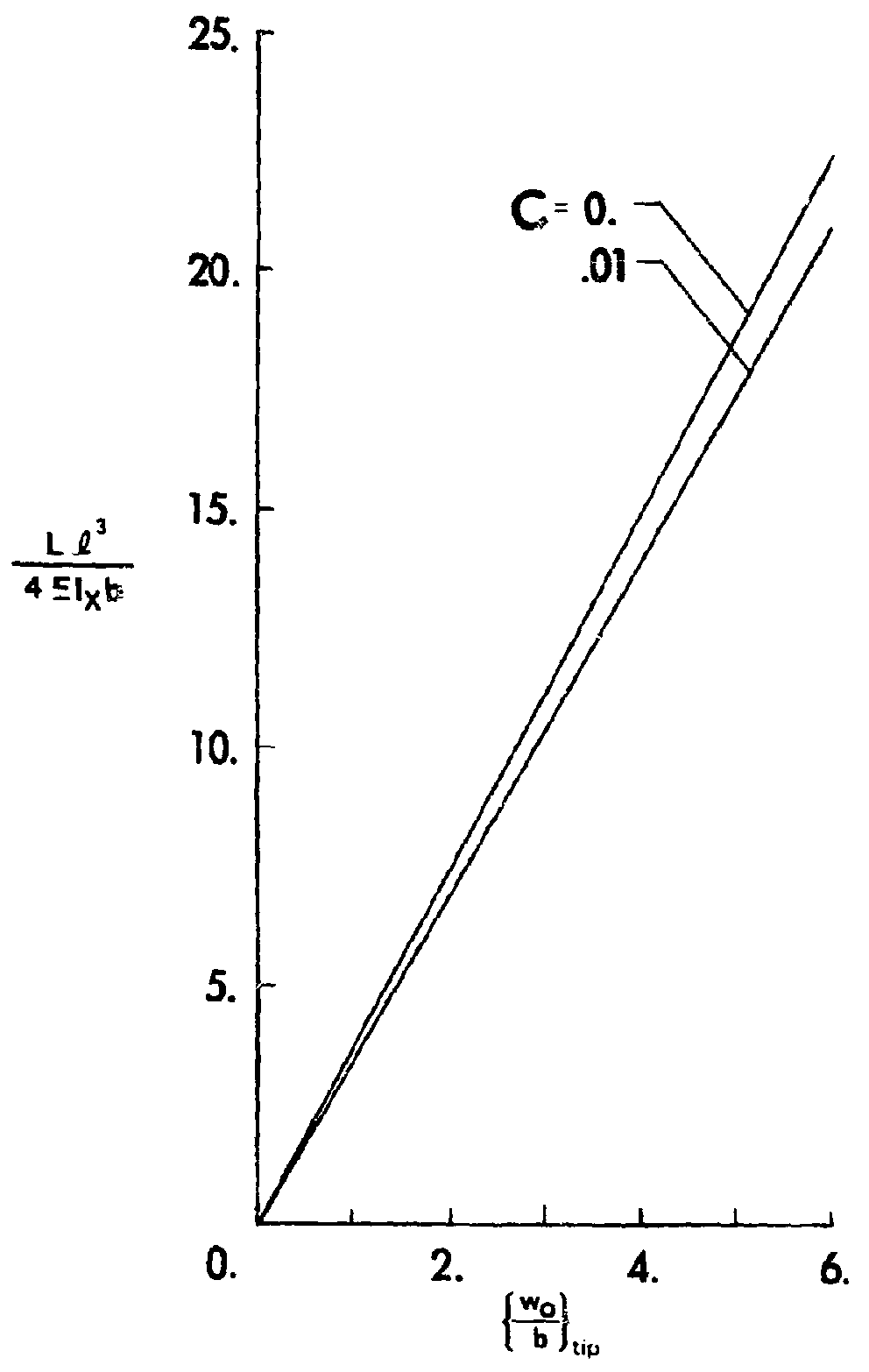

FIGURE 6-8 Effect of Drag on the Relation Between Steady Lift and Steady Bending Deflection, For the Fig. 6-7 Flutter Conditions 


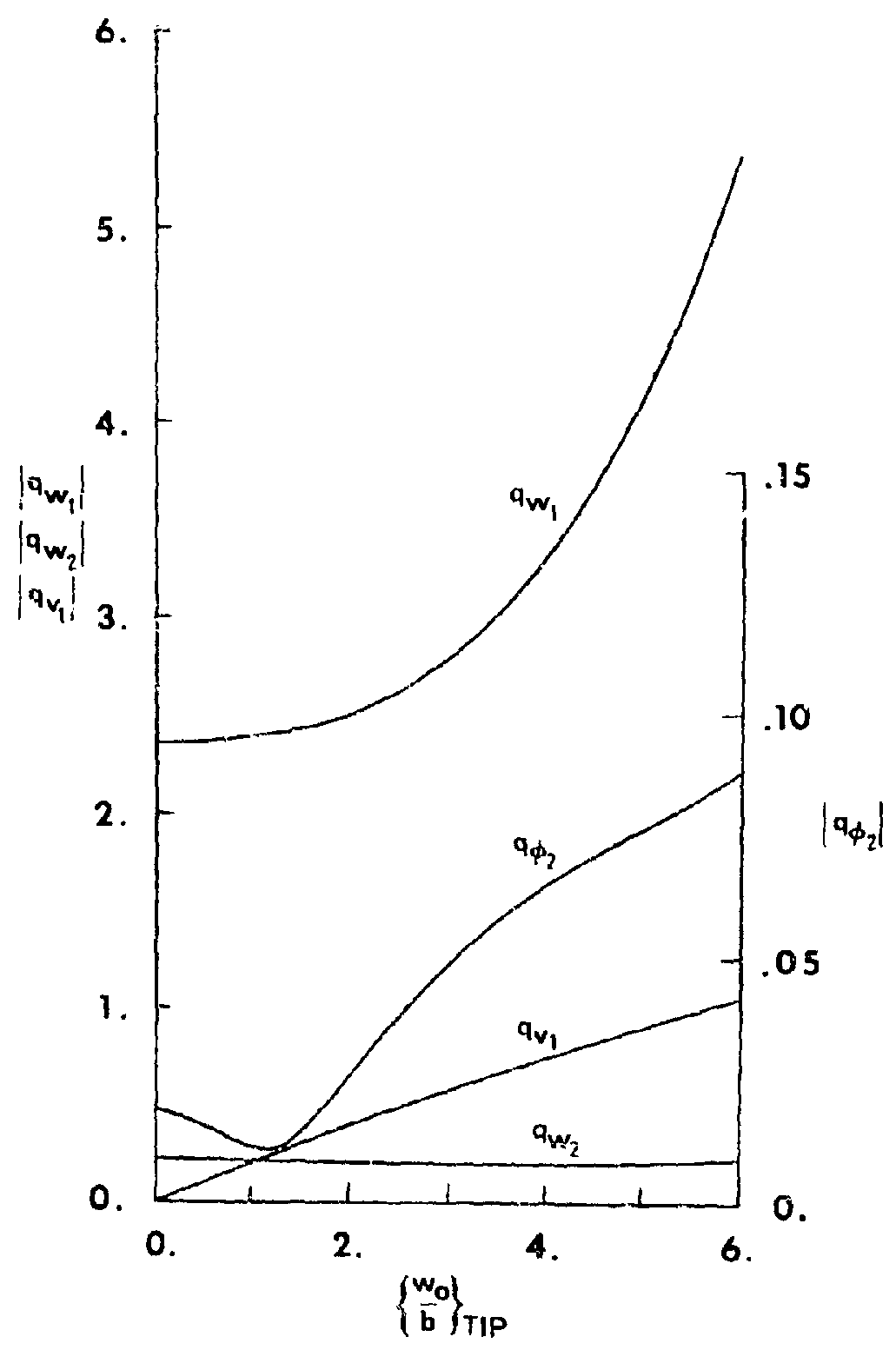

FIGURE 6-9 Elutter Mode Shape Amplitudes for Unit $\mid q_{\alpha_{1}}$, for the
Fig. 6-7 Stability Boundary With $\mathrm{C}=0$ 


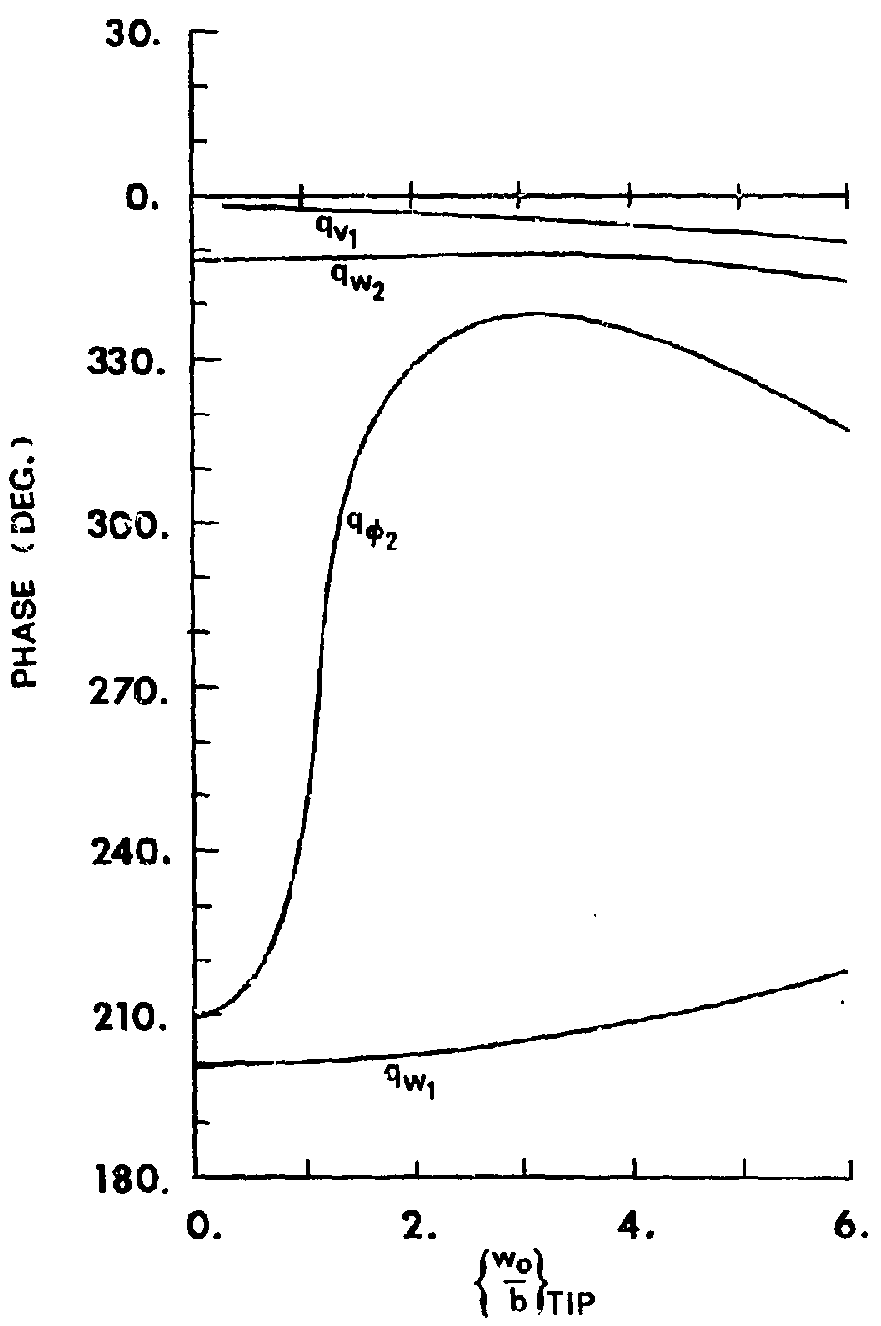

FIGURE 6-10 Flutter Mode Shape Phase Relations for Zero Phase of $q_{\phi_{1}}$,
far the Fig. 6-7 Stability Boundary With $\mathrm{C}=0$ 


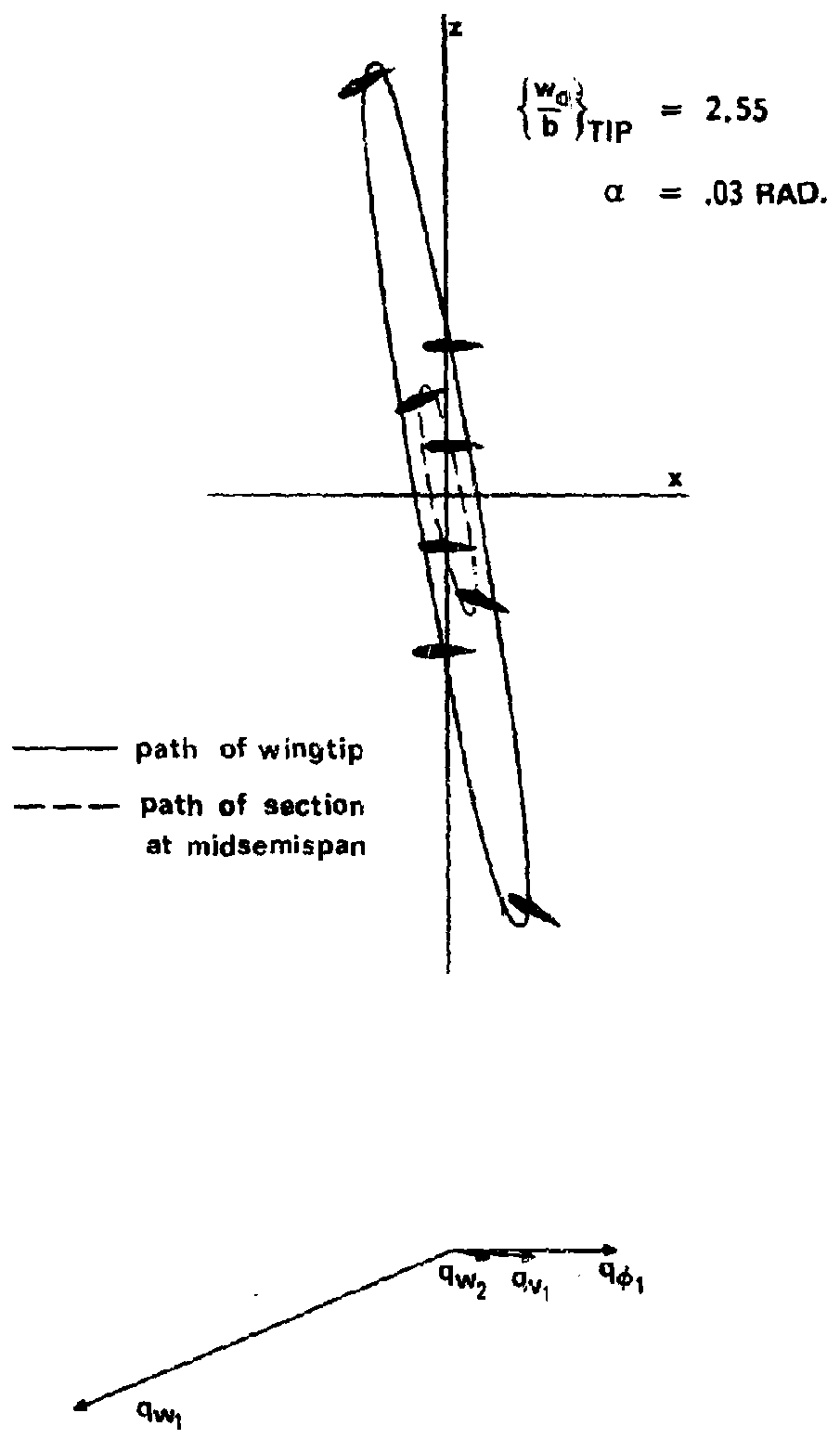

FIGURE 6-11 Physical Appearance of Flutter Mode of Deflected Wing; Fig. $6-7$ Configuration with $\mathrm{C}=0$ 

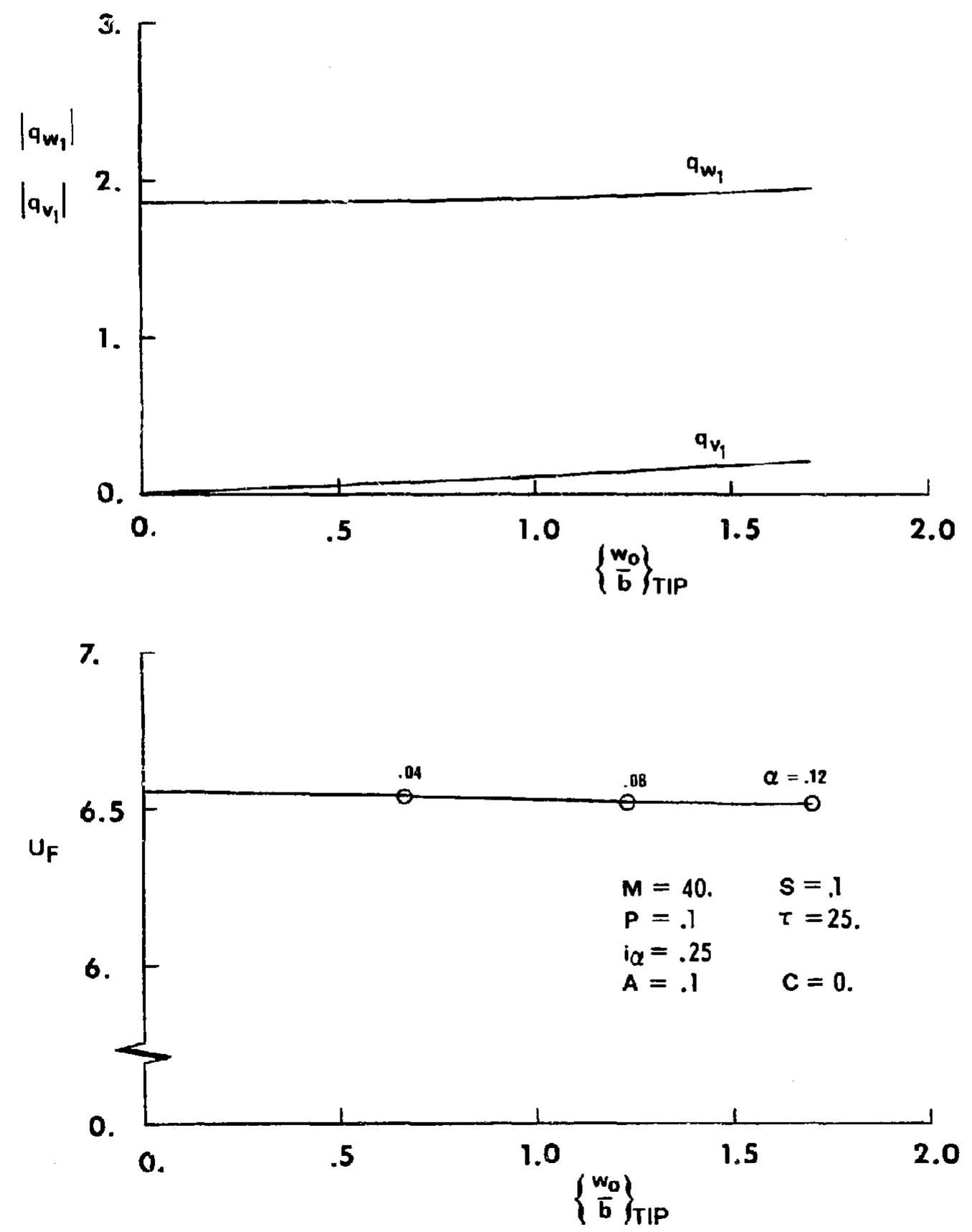
FIGURE 6-12 Flutter Speed and Mode Shape Amplitudes, for Unit $\left|q_{\phi_{1}}\right|$,
As Affected By Steady Deflections; Low-Aspect-Ratio Example 


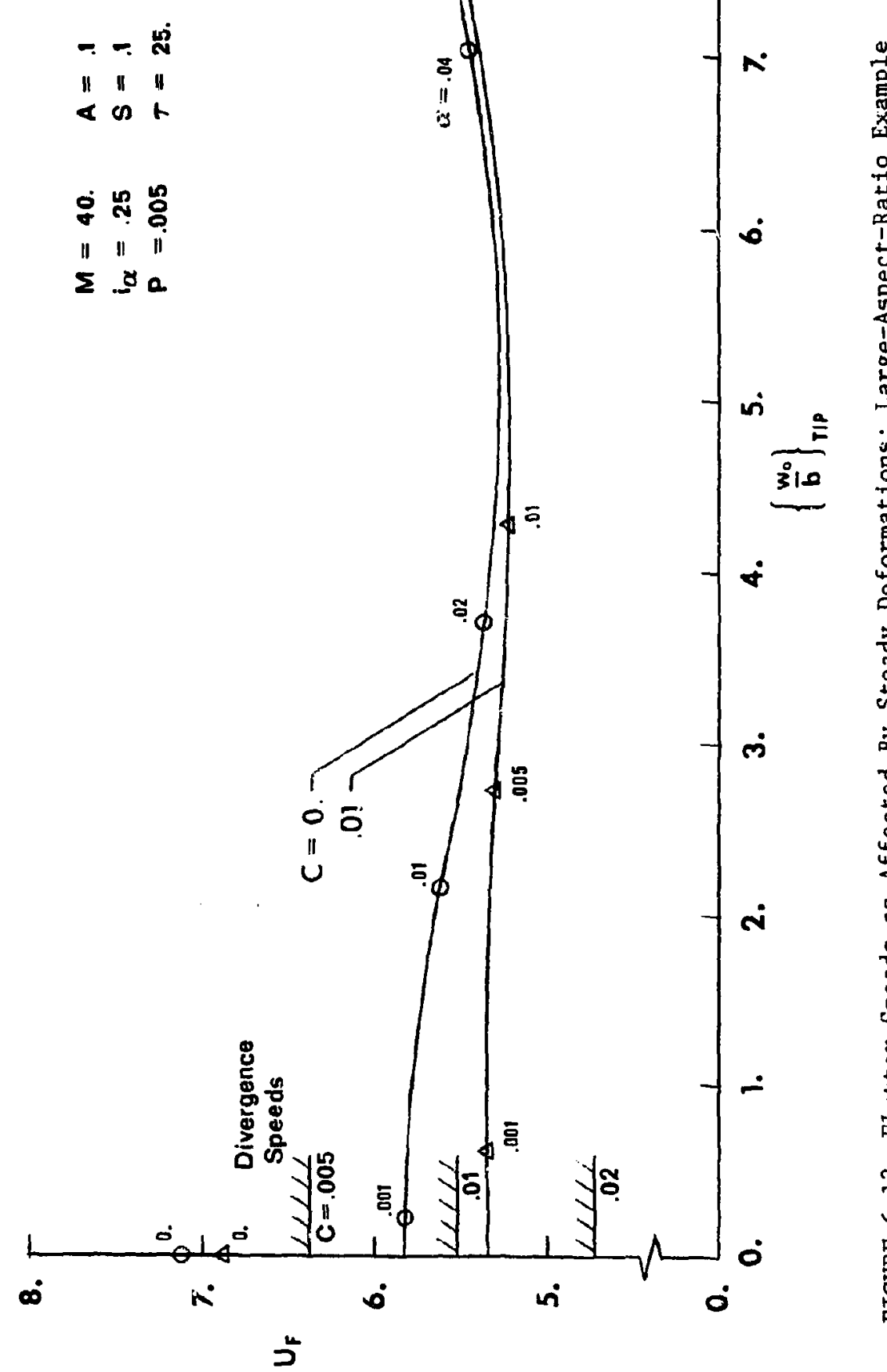

吾 


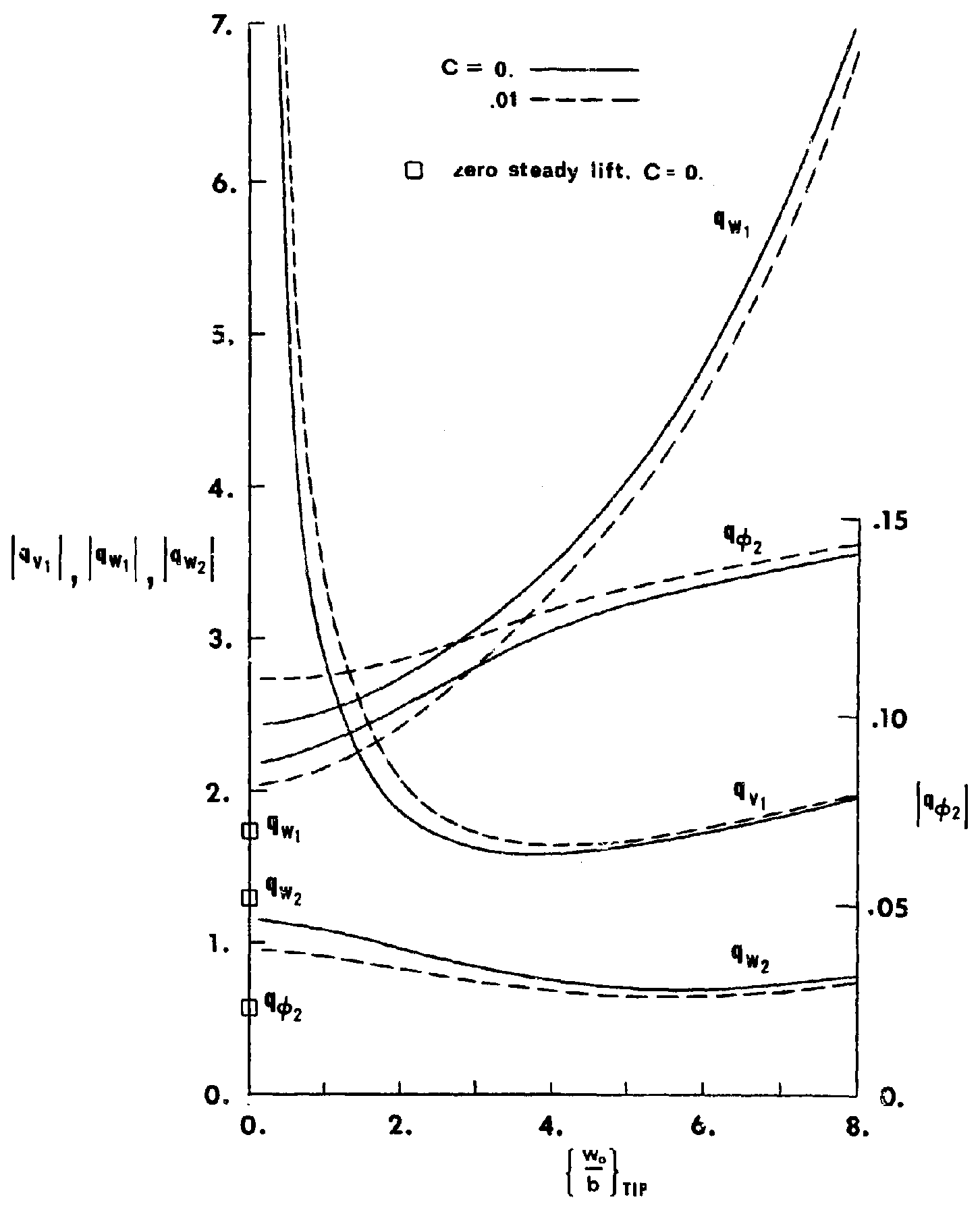

FIGURE 6-14 Flutter Mode Shape Amplitudes for Unit $\left|q_{\phi_{1}}\right|$ Corresponding
to Fig. 6-13 


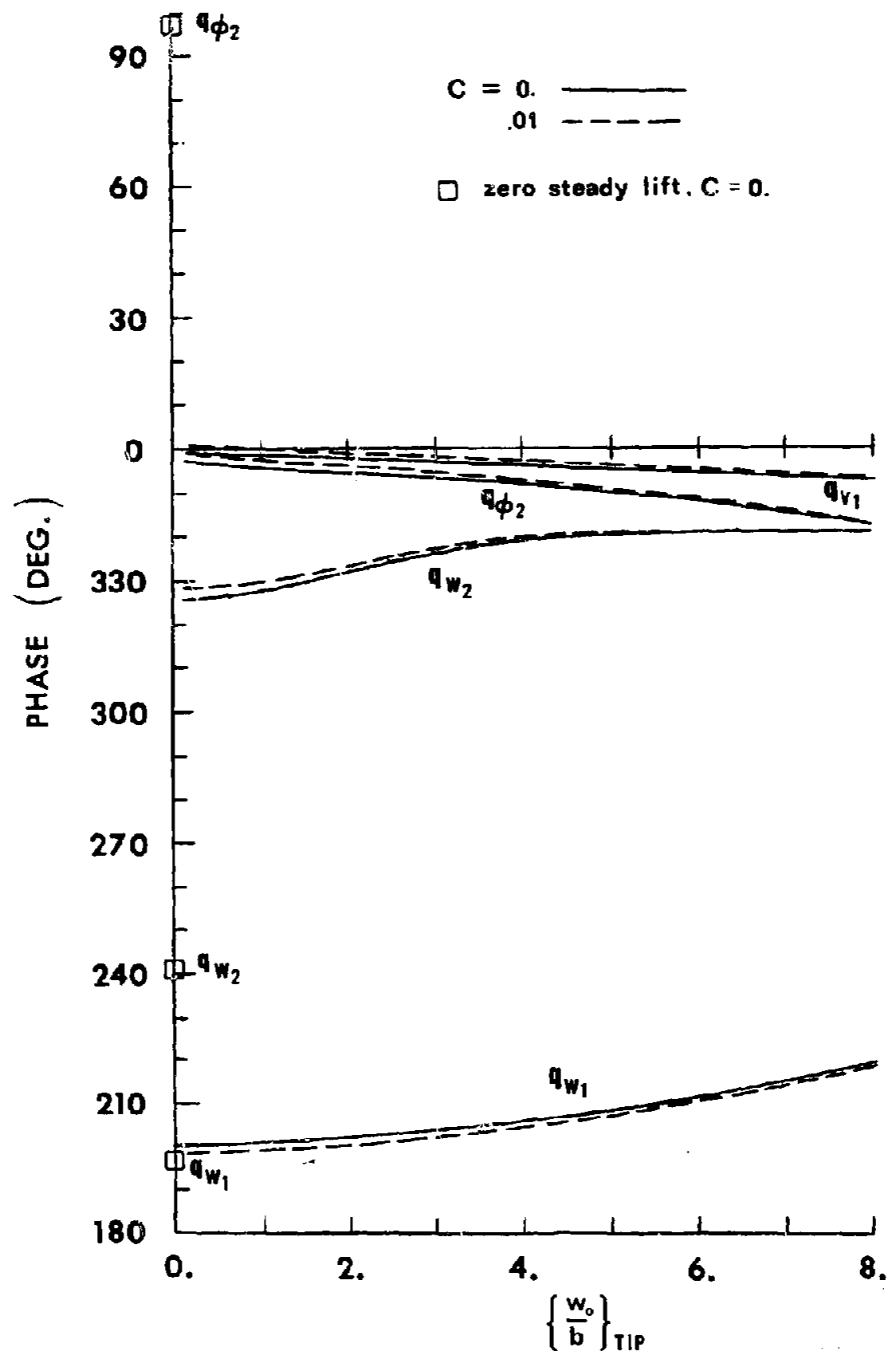

FIGURE 6-15 Flutter Mode Shape Phase Relations for Zero Phase of $q_{\phi_{1}}$
Corresponding to Fig. $6-13$ 

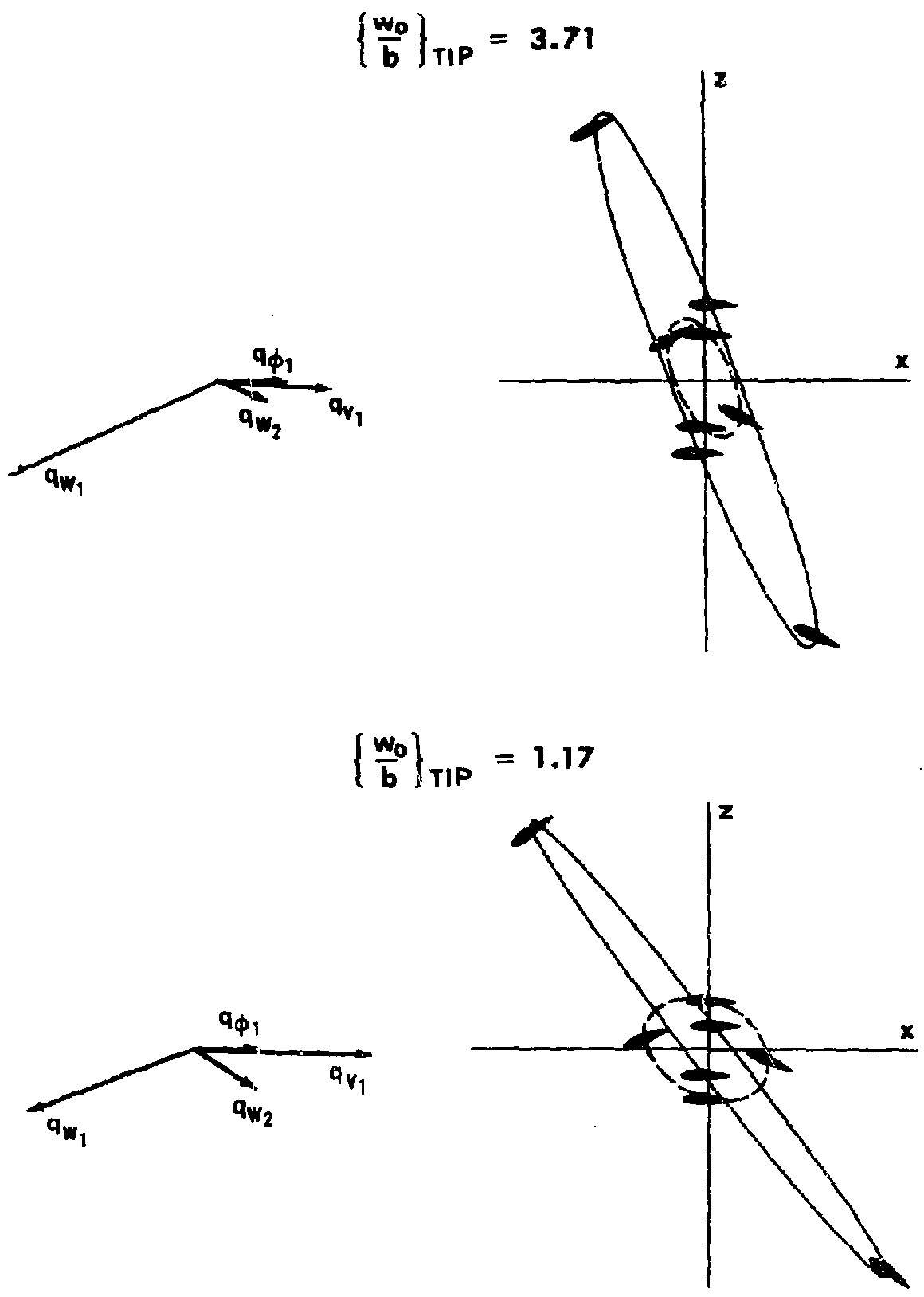

path of wingtip

- path of section at $\tilde{y}=.5$

FIGURE 6-16 Physical Appearance of Flutter Mode of Deflected High Aspect Ratio Wing of Fig. 6-13 


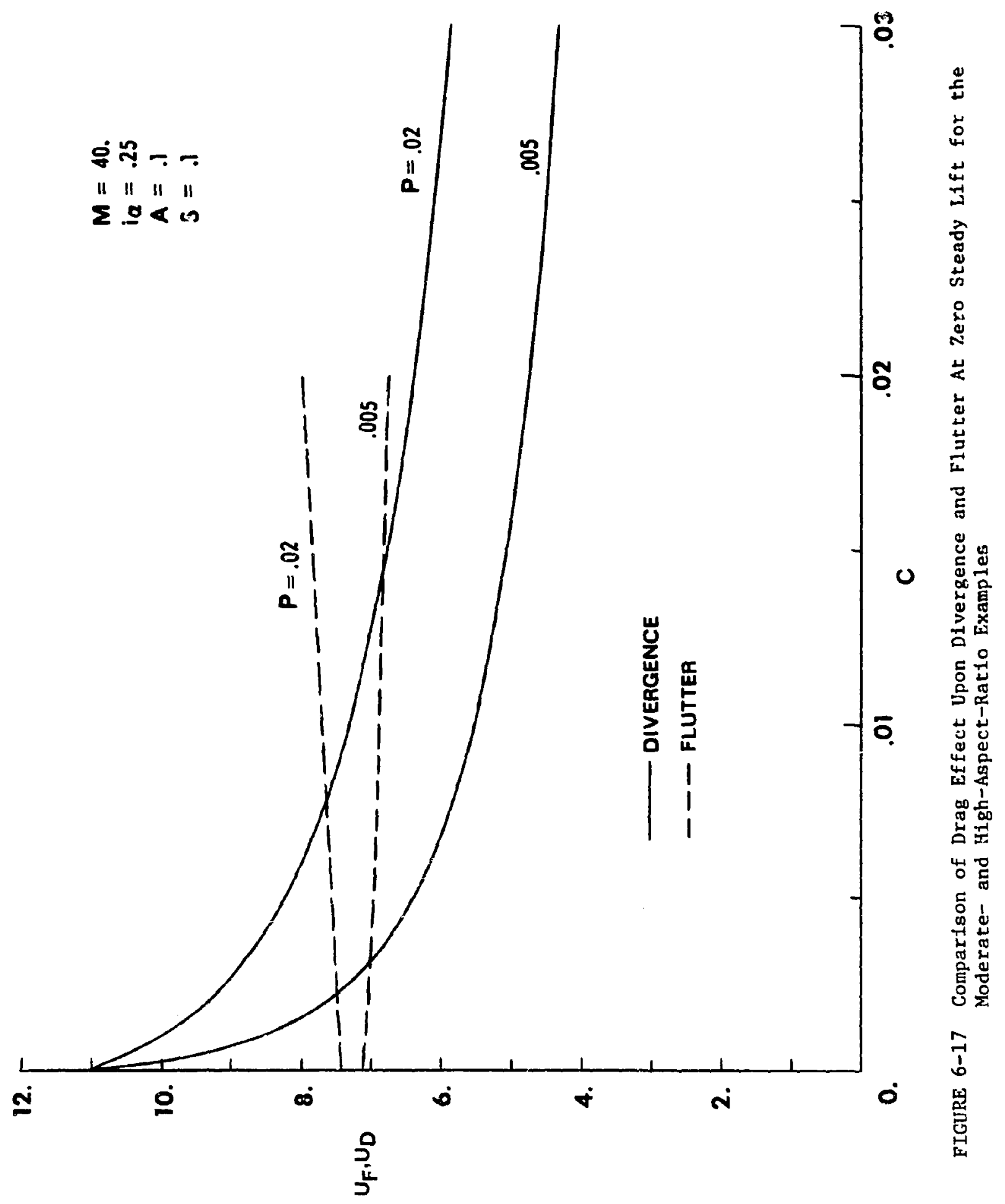




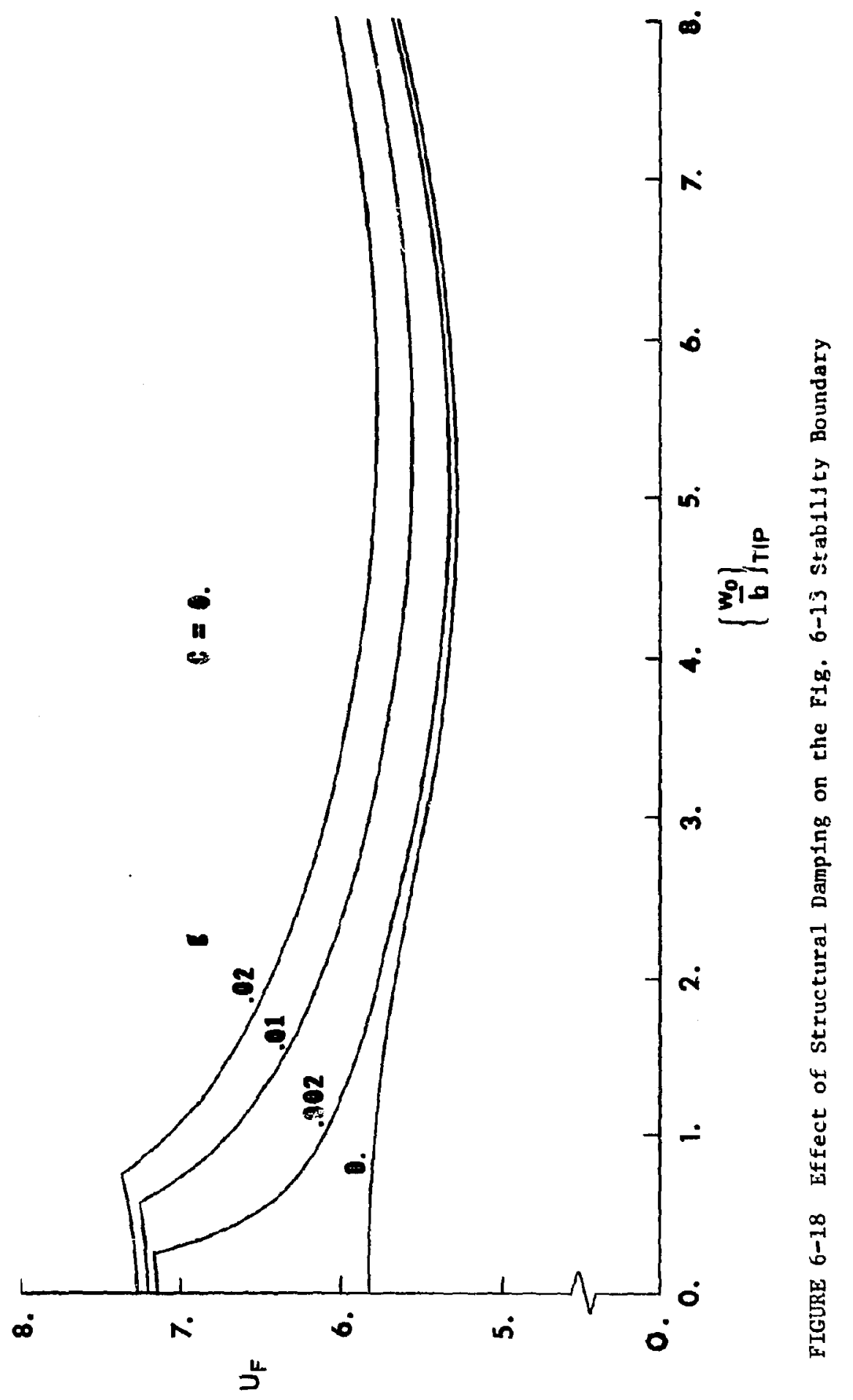




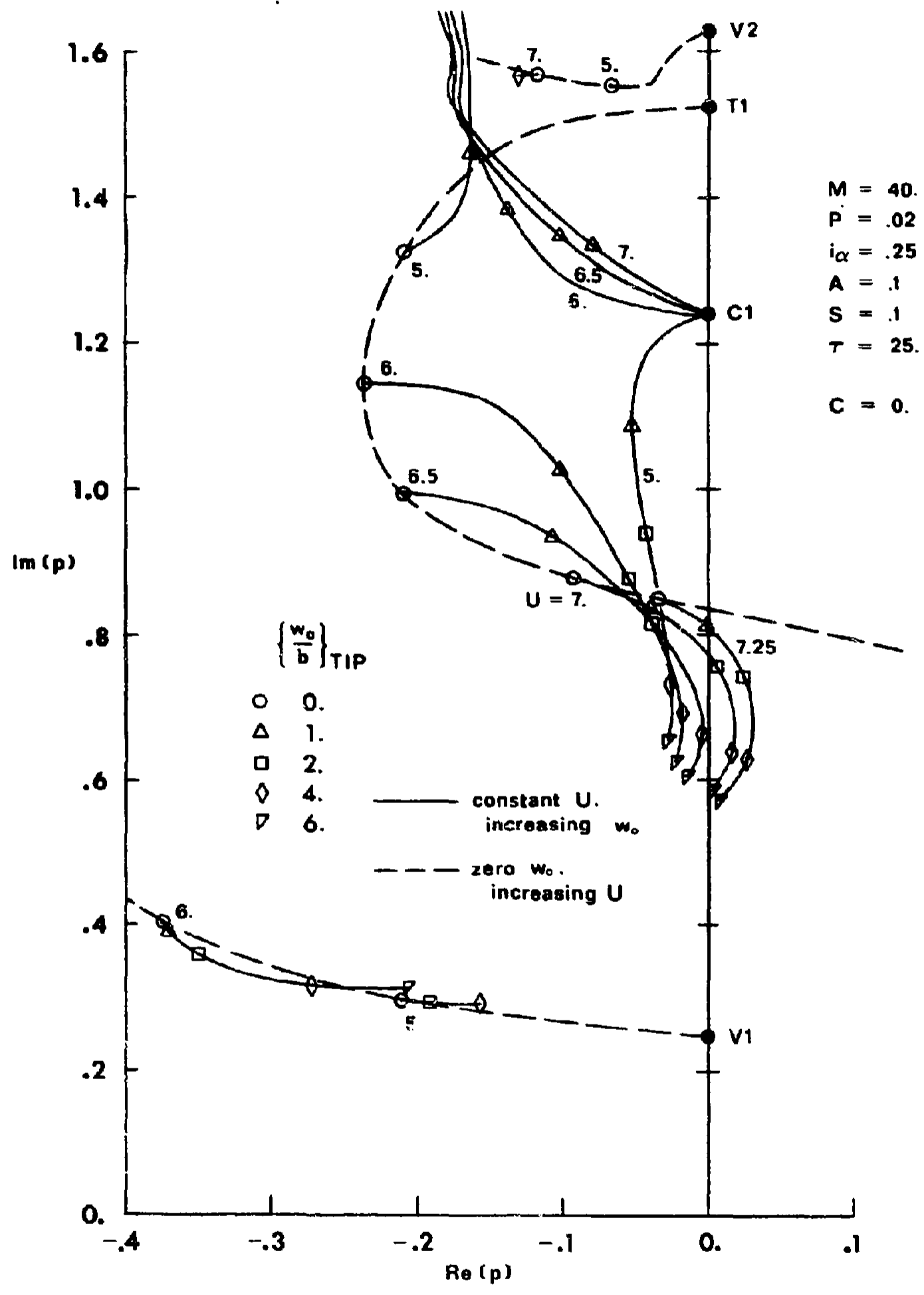

FIGURE 6-19 Locus of Roots for True Aeroelastic Modes, ModerateAspect-Ratio Example 


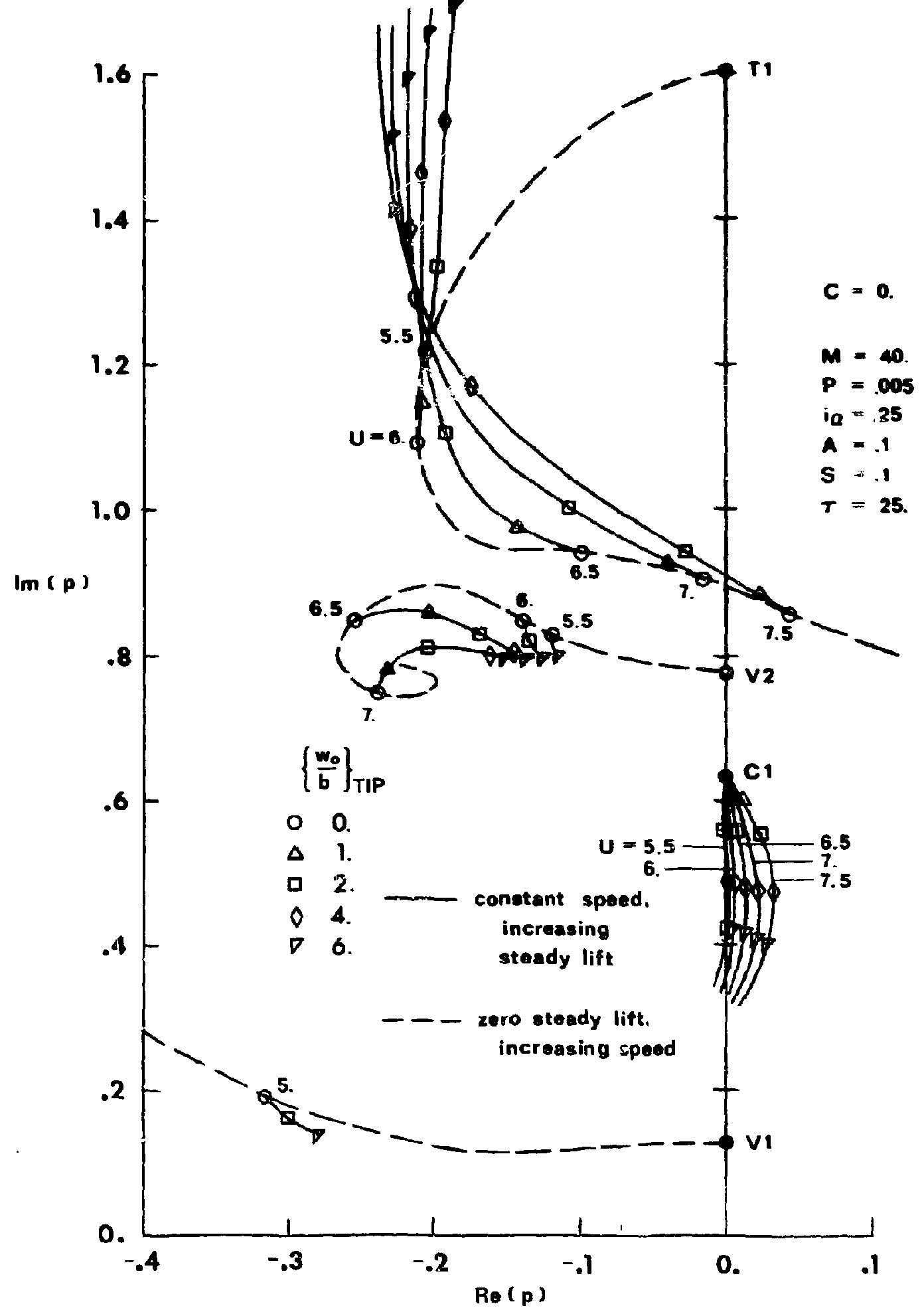

FIGURE 6-20 Locus of Roots for True Aeroelastic Modes, High-Aspect-Ratio Example 


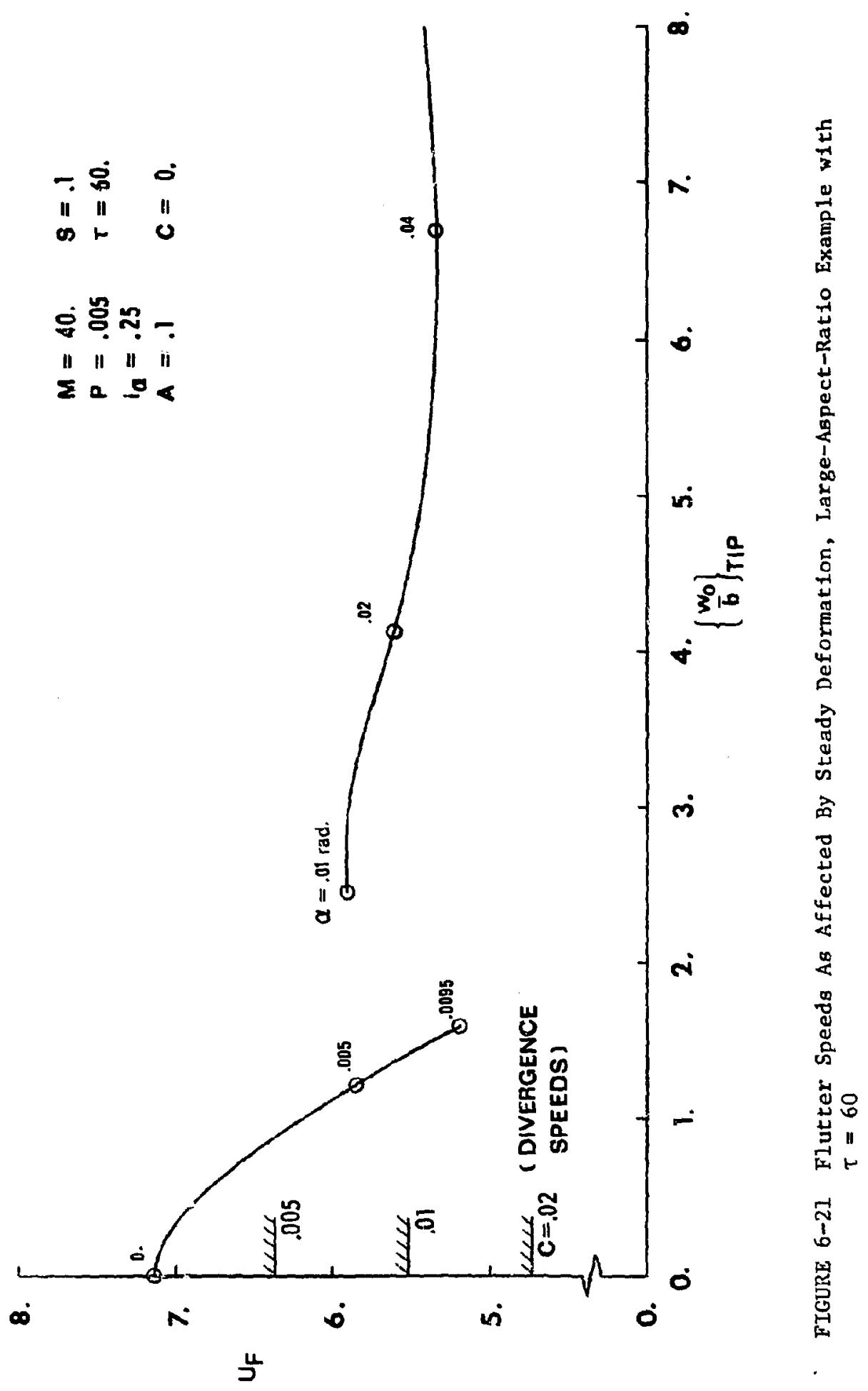




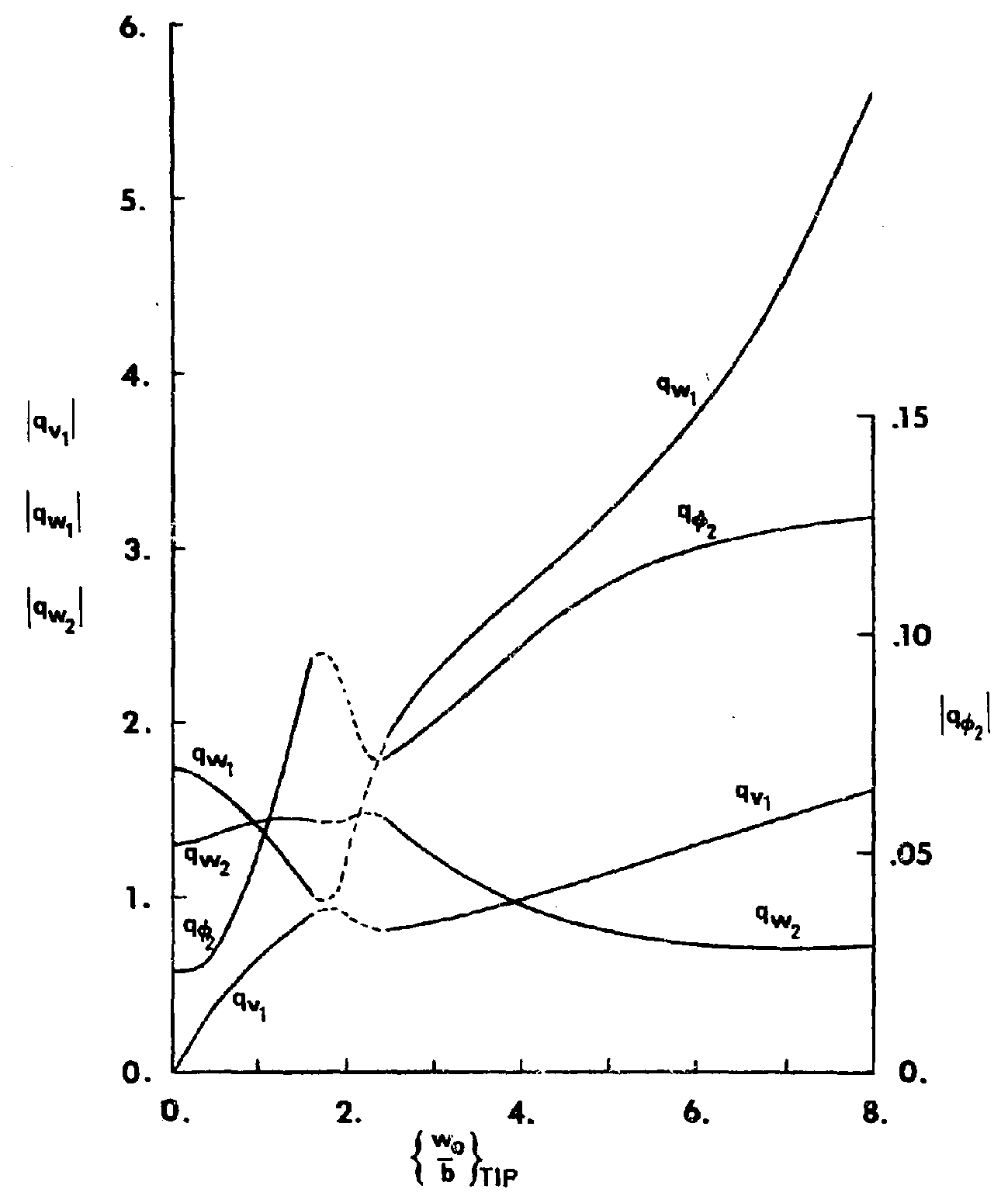

FIGURE 6-22 Flutter Mode Shape Amplitudes for Unit $\left|q_{\phi_{1}}\right|$ Corresporiding
to Fig. 6-21 


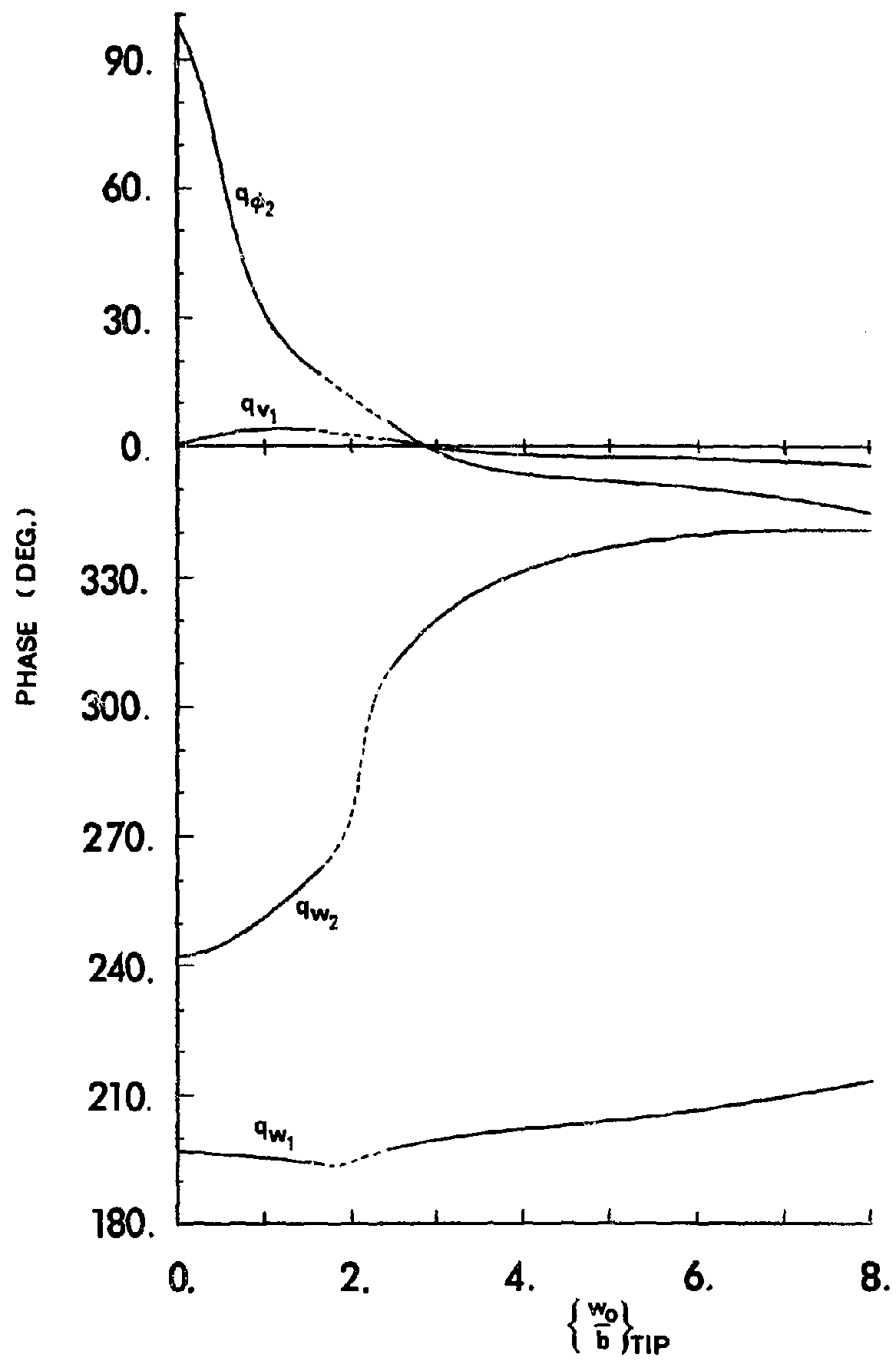

FIGURE 6-23 Flutter Mode Shape Phase Relations for Zero Phase of $\mathbf{q}_{\phi_{1}}$,
Corresponding to Fig. $6-21$ 


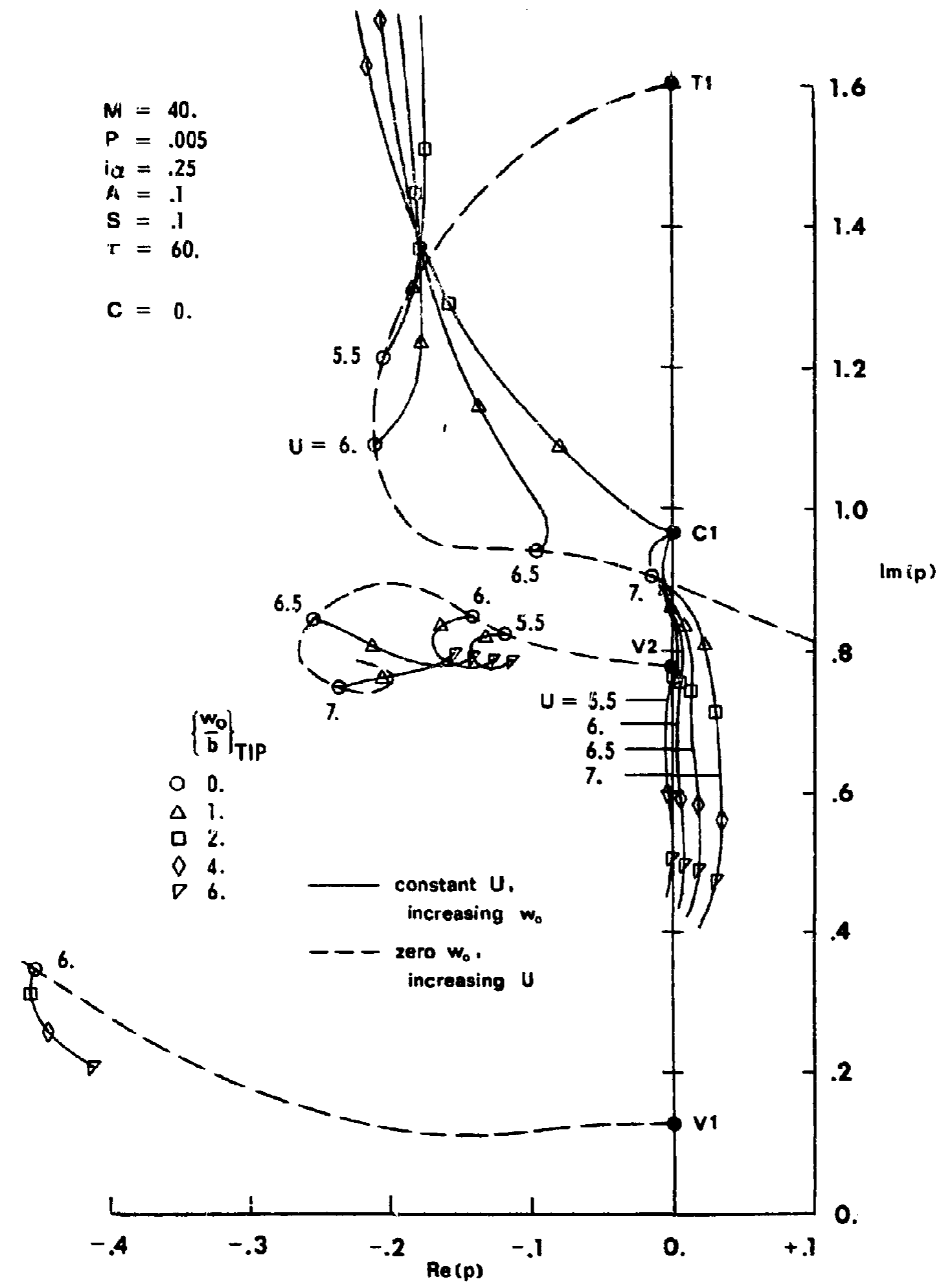

Figure 6-24(a) Locus of Roots for True Aeroelastic Misdes, LargeAspect-Ratio Example with $\tau=60$ 


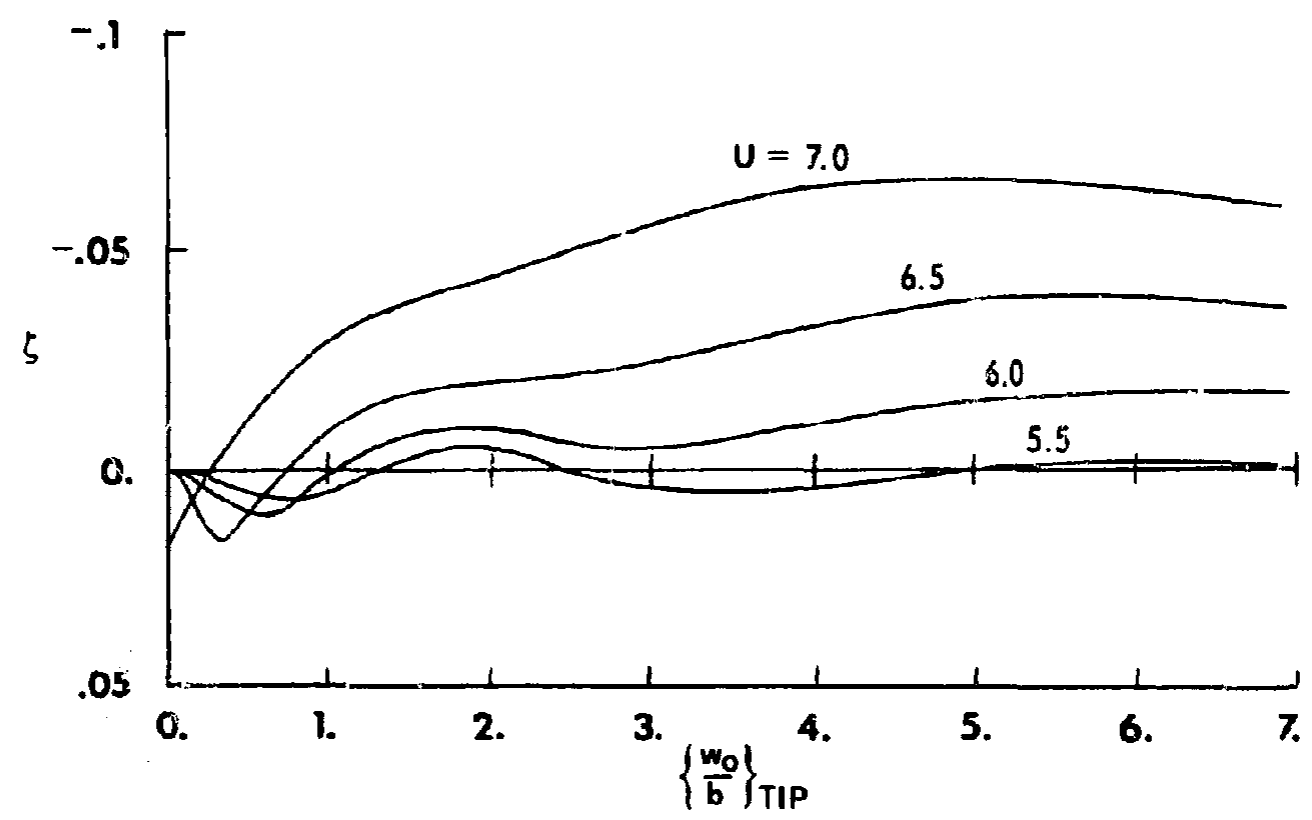

FIGURE 6-24(b) Damping Ratio of Flutter Mode Constant Speed Branches

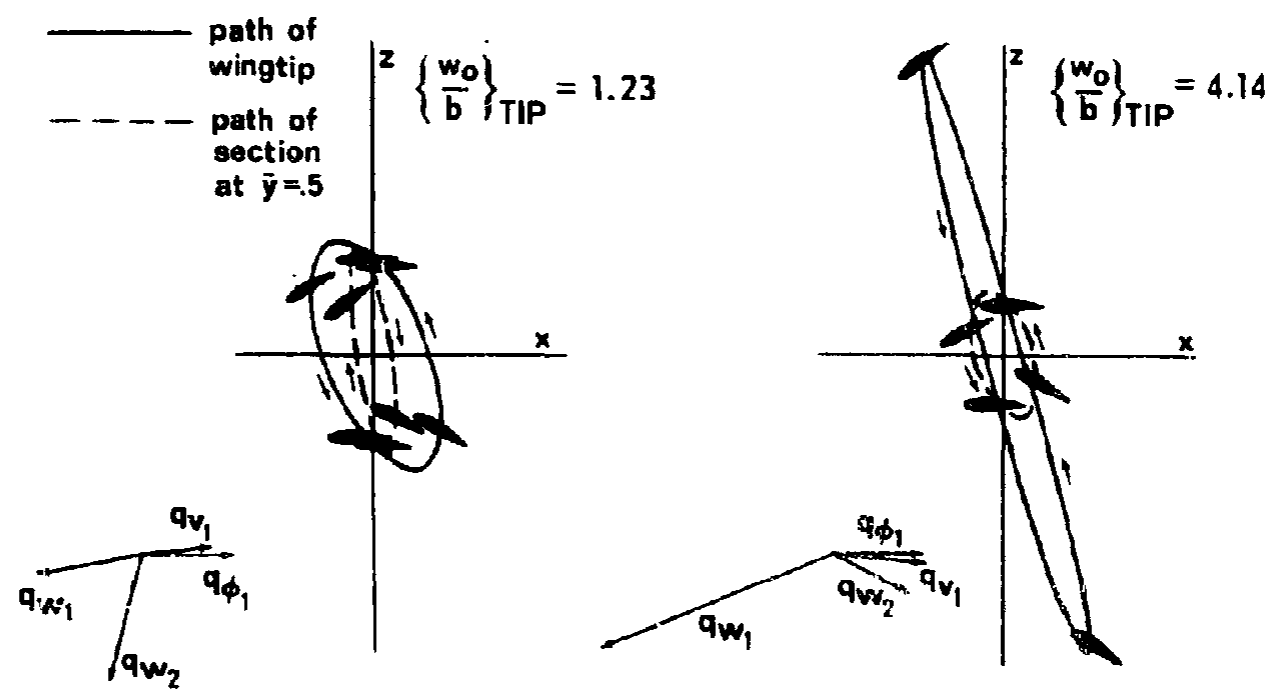

FIGURE 6-25 Physical Appearance of Flutter Modes Defined in Figs, 6-22, 6-23 

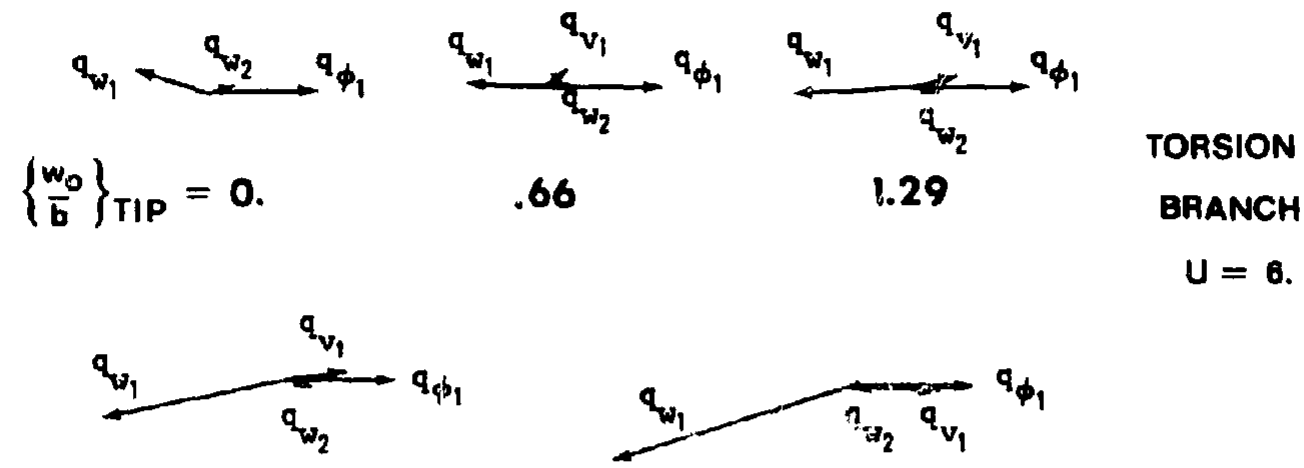

2.44

3.87

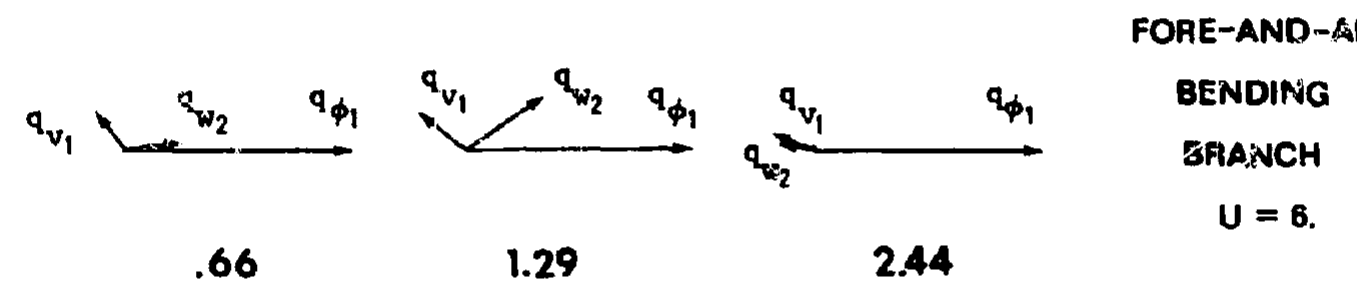

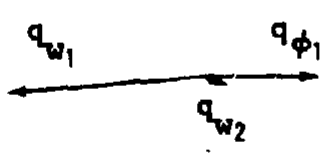

0.

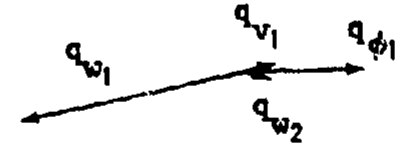

1.05
TORSION

BRANCH

$\mathbf{U}=\mathbf{7}$.
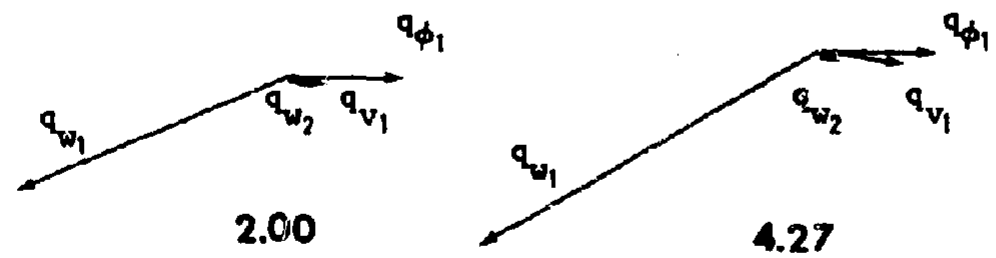

FIGURE 6-26 Mode Shapes for Selected Roots from FIg. 6-19 


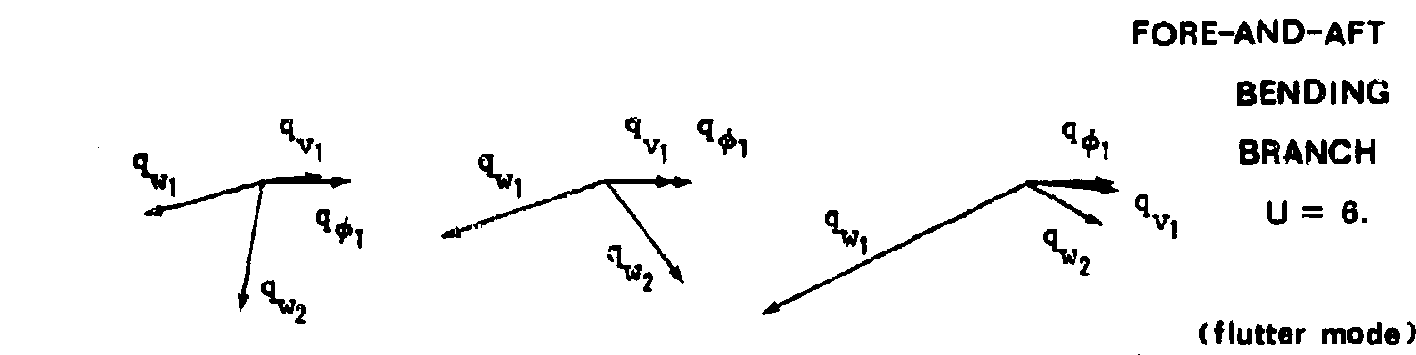
$\left\{\frac{\omega_{0}}{b}\right\}_{\text {TIP }}=1.31$
2.57
5.29
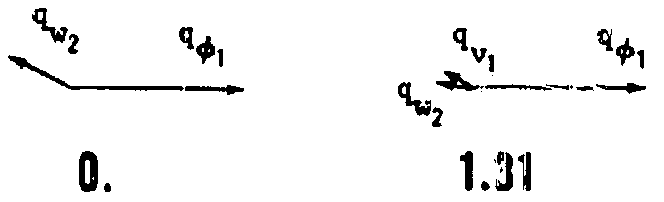

TORSION

BRANCH

$j=8$.
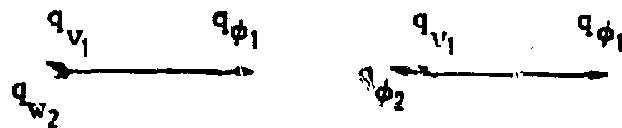

2.57

5.29

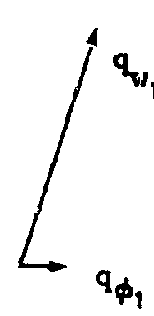

0.

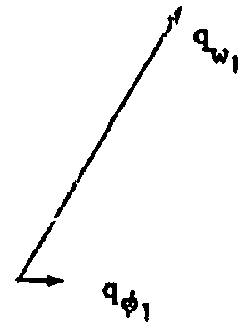

2.57

FIGURE 6-27(a) Mode Shapes for Salected Roots from Fig - 6-24 

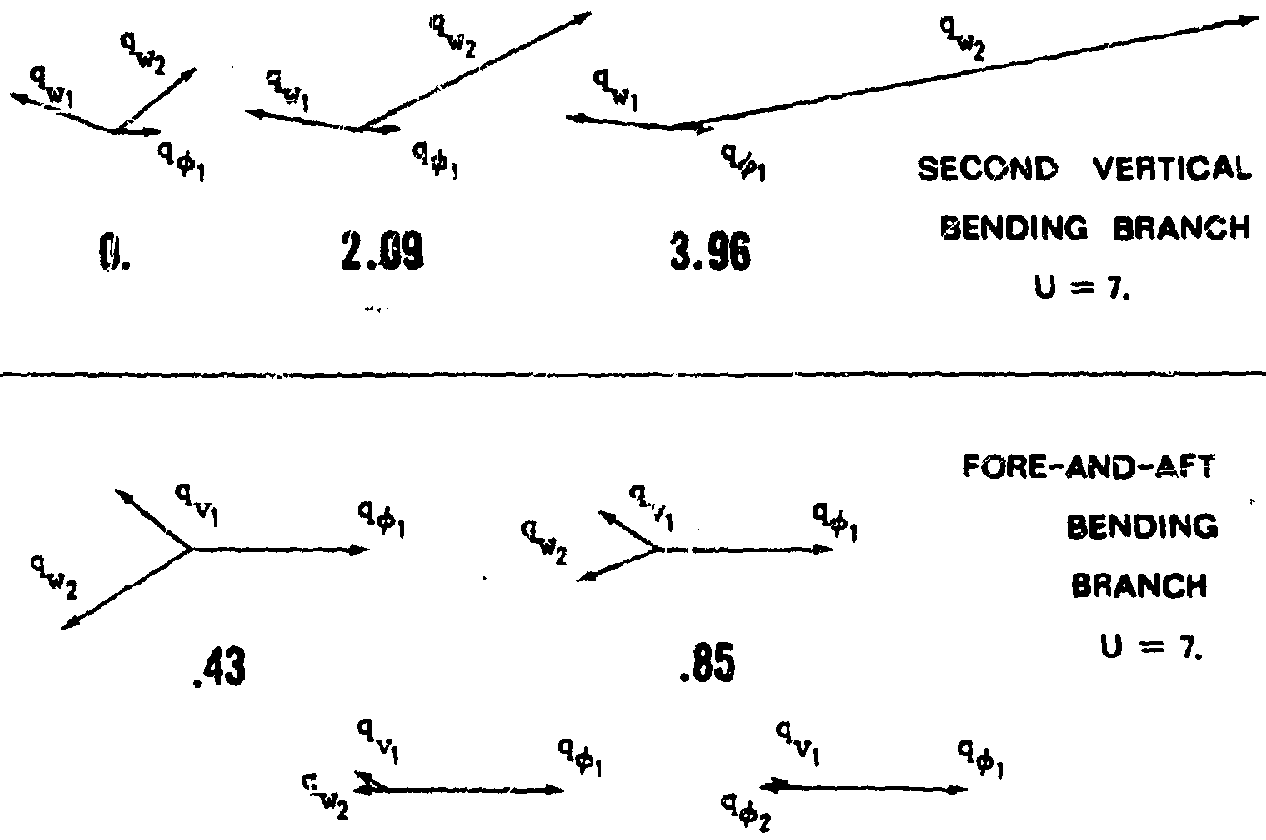

2.09

3.96

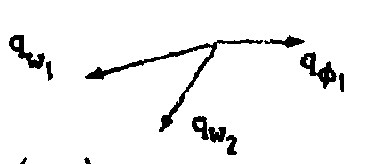

$\left\{\begin{array}{c}w_{0} \\ b\end{array}\right\}_{T I P}=0$.

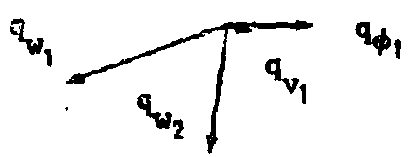

.85

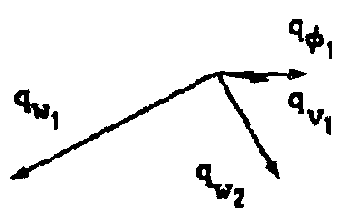

2.09

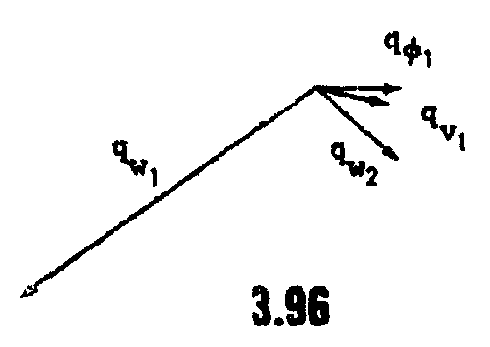

(fiutter mode)

FIGURE $6-27(b)$ 


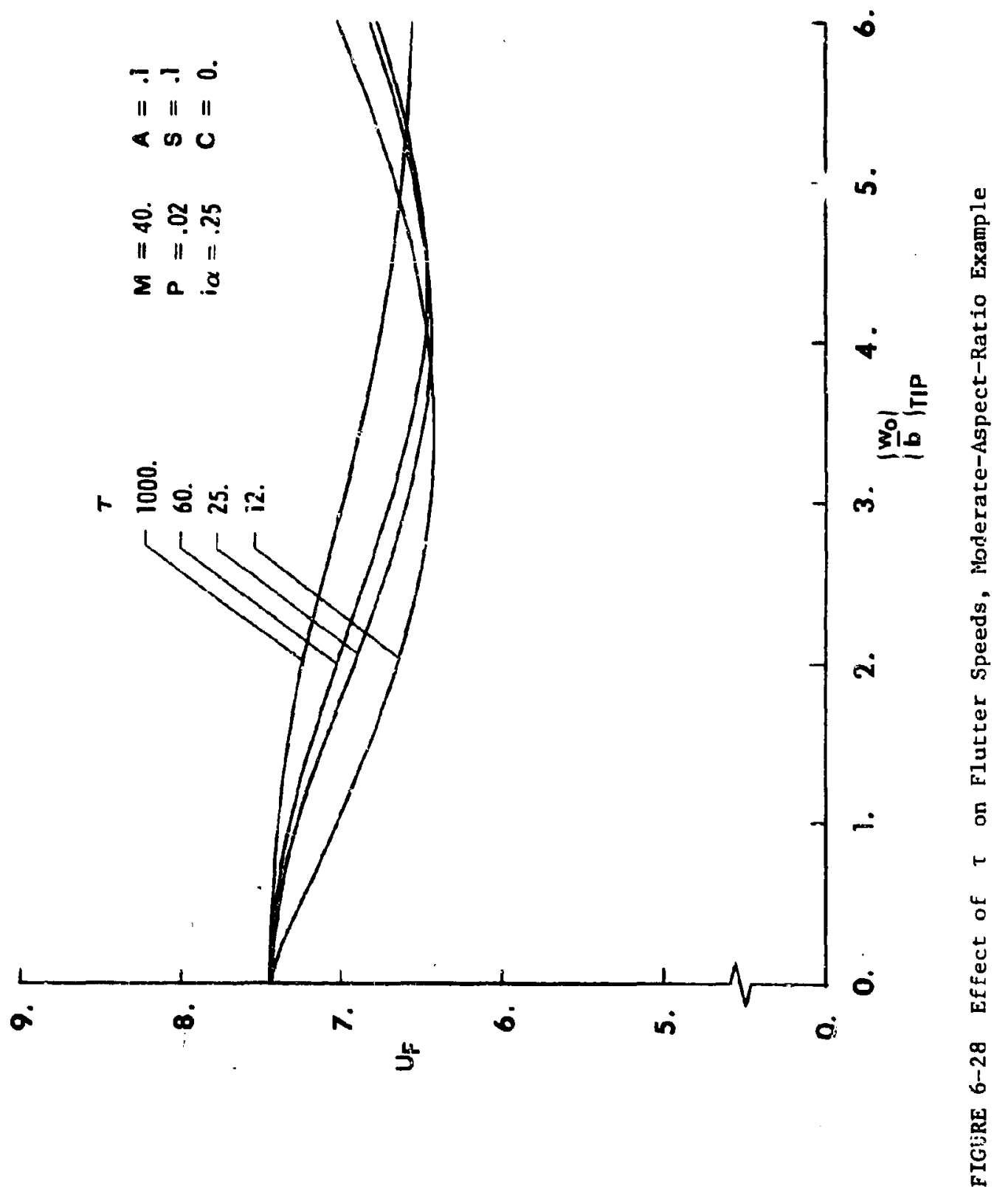

182 


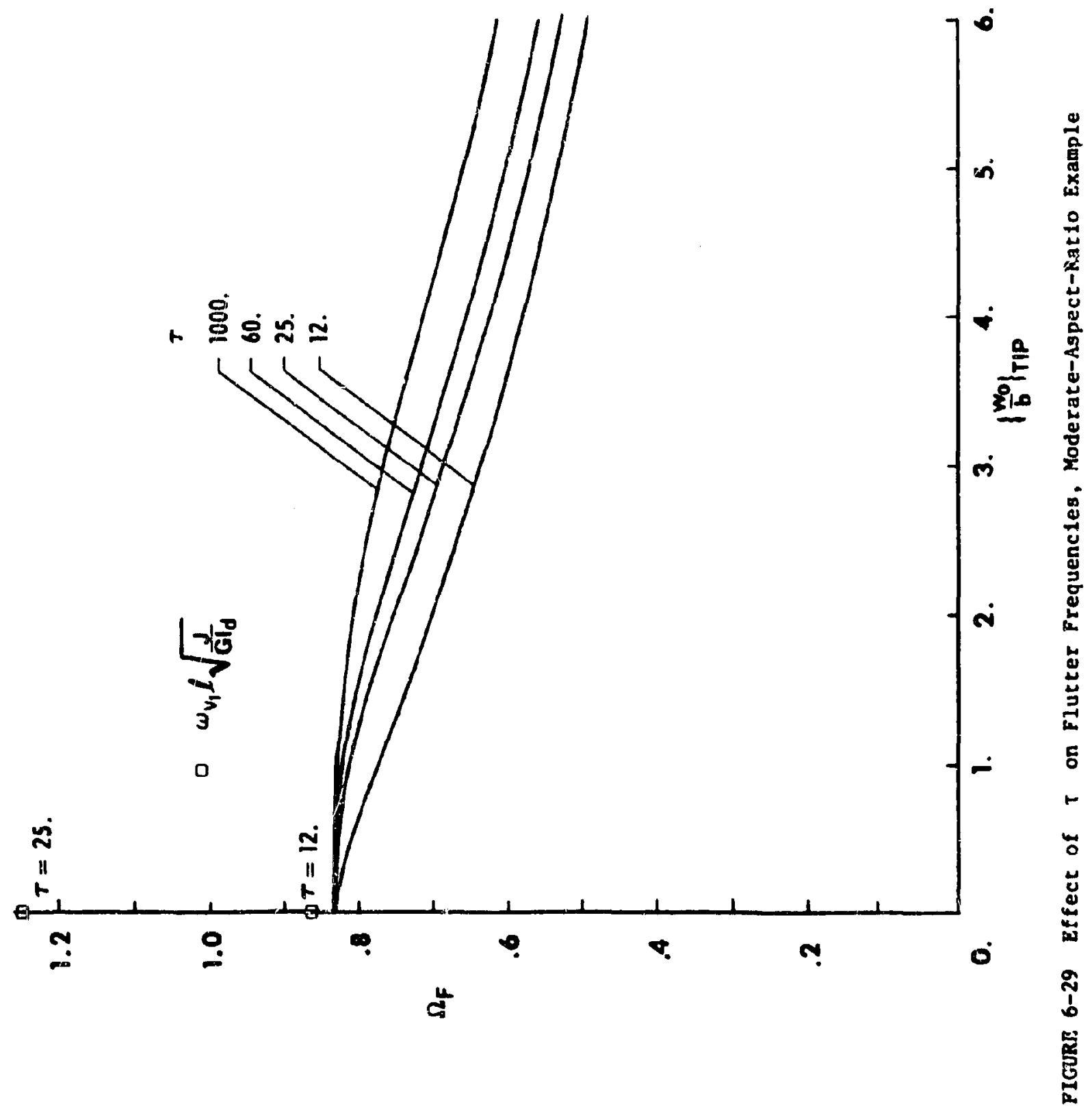




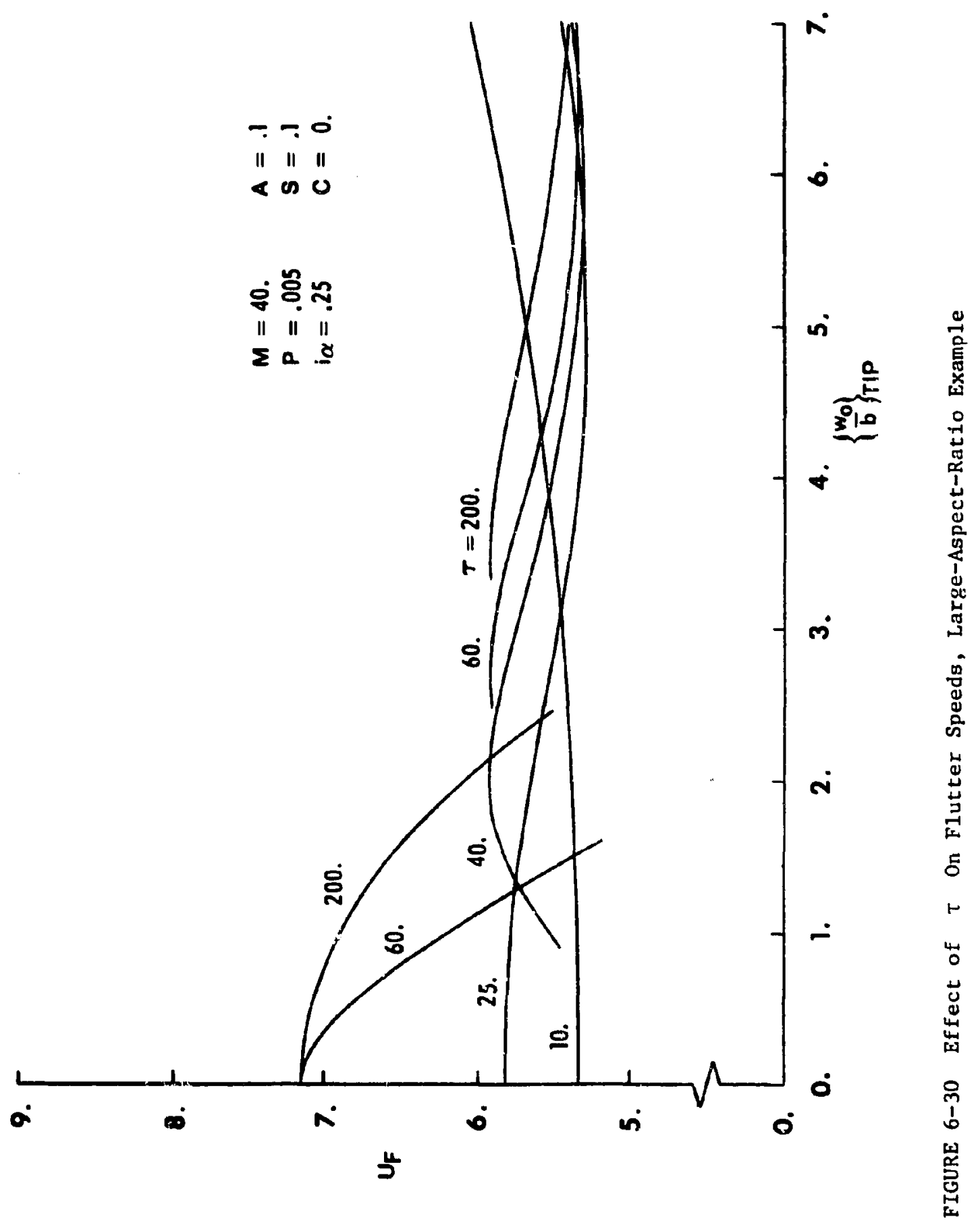




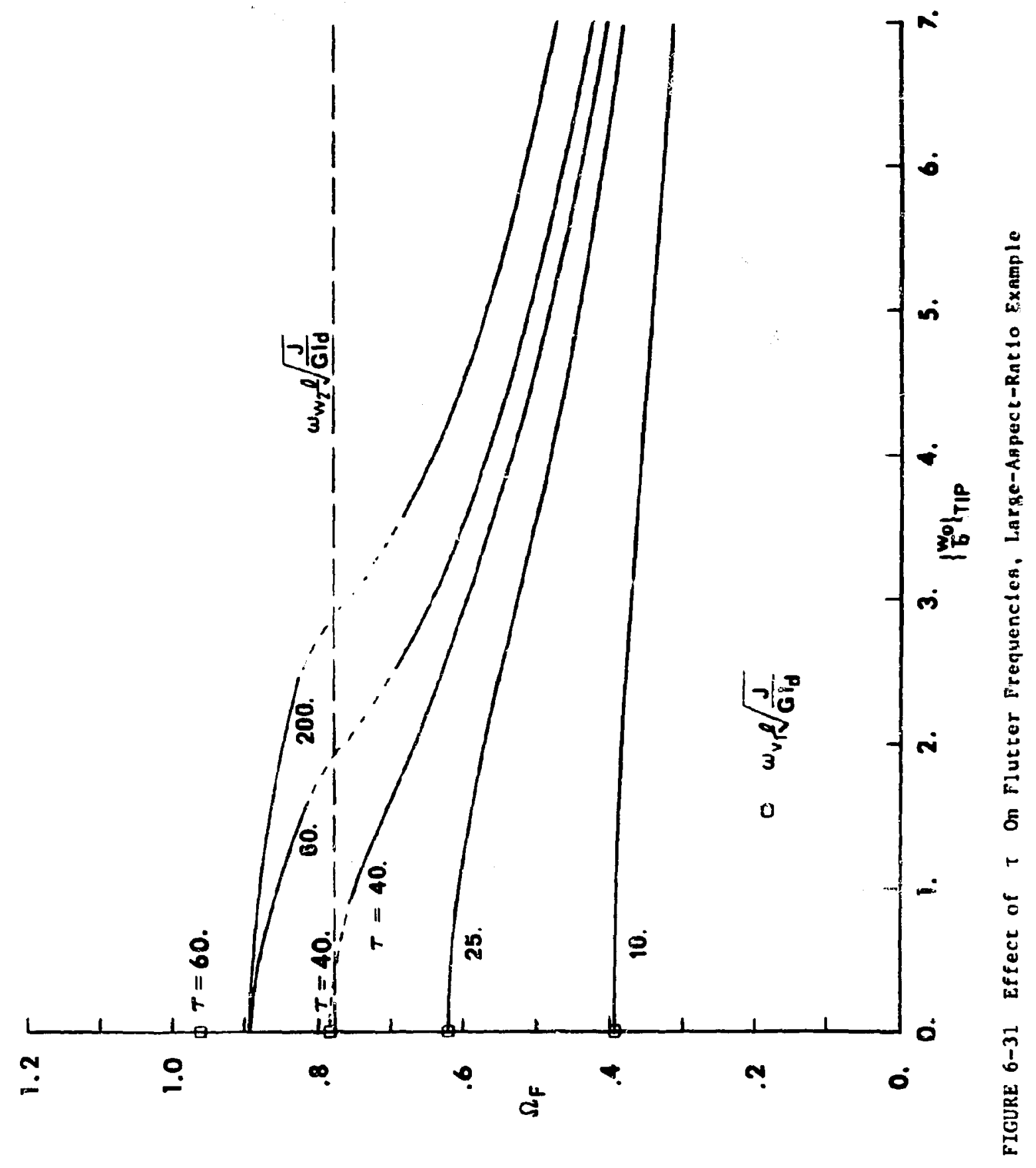




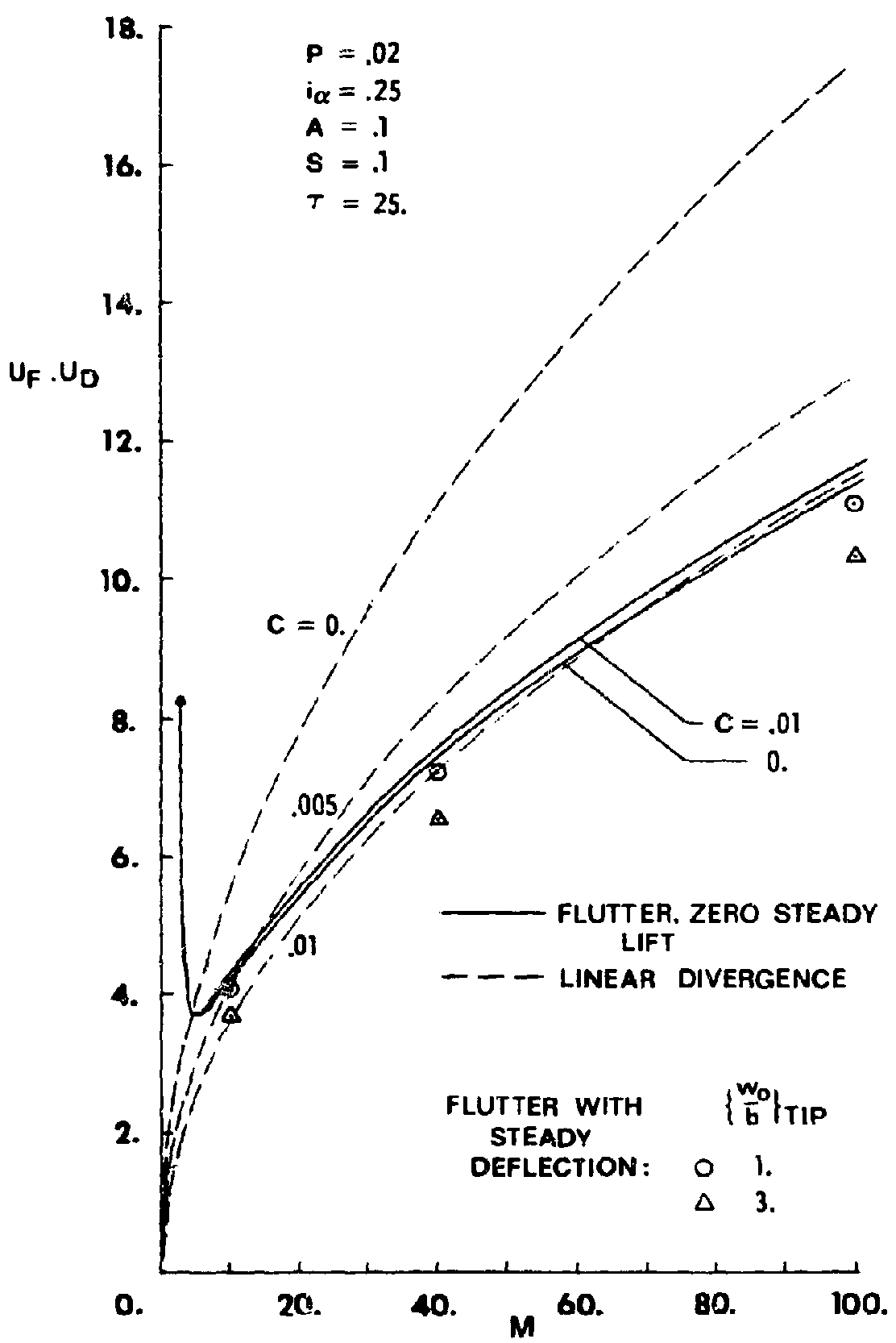

Figure 6-32 Effect of Mass Ratio on Divergence and Flutter, ModerateAspect-Ratio Example 


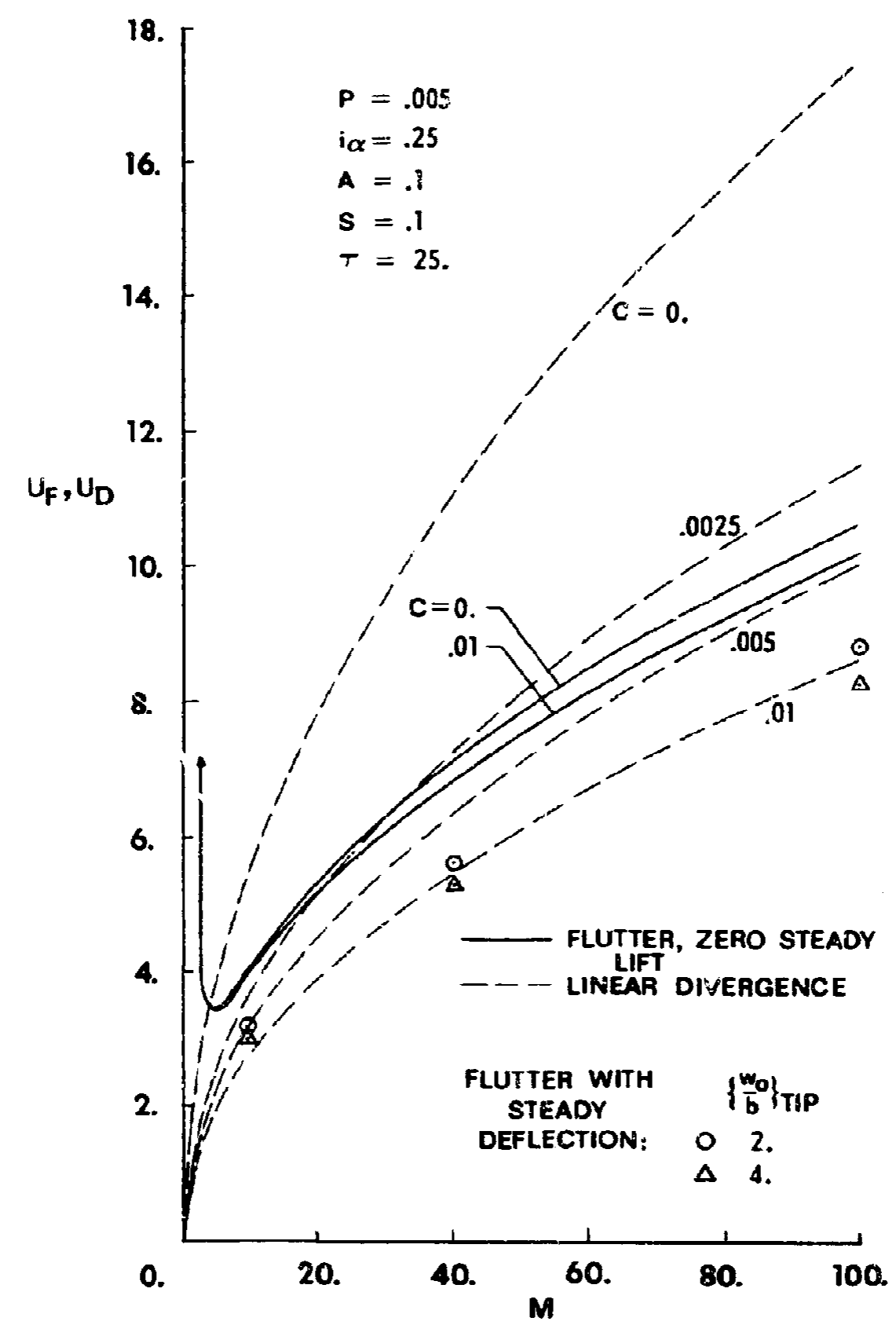

FIGURE 6-33 Effect of Mass Ratio on Divergence and Flutter, vargeAspect-Ratio Example 


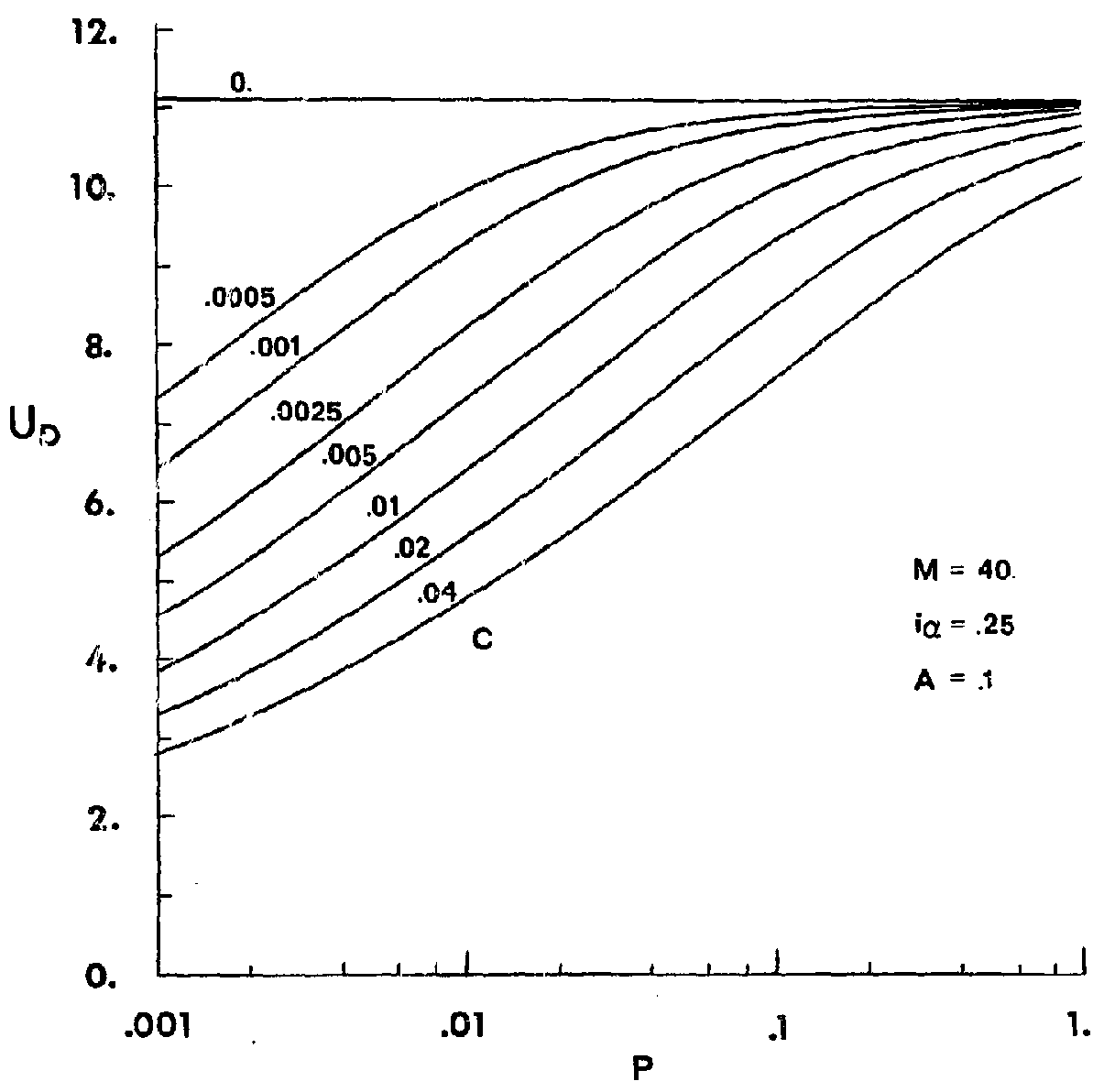

FIGURE 6-34 Divergence Speeds for Selected Values of Drag Parameter $C$ as Aspect Ratio Parameter $P$ Varies 


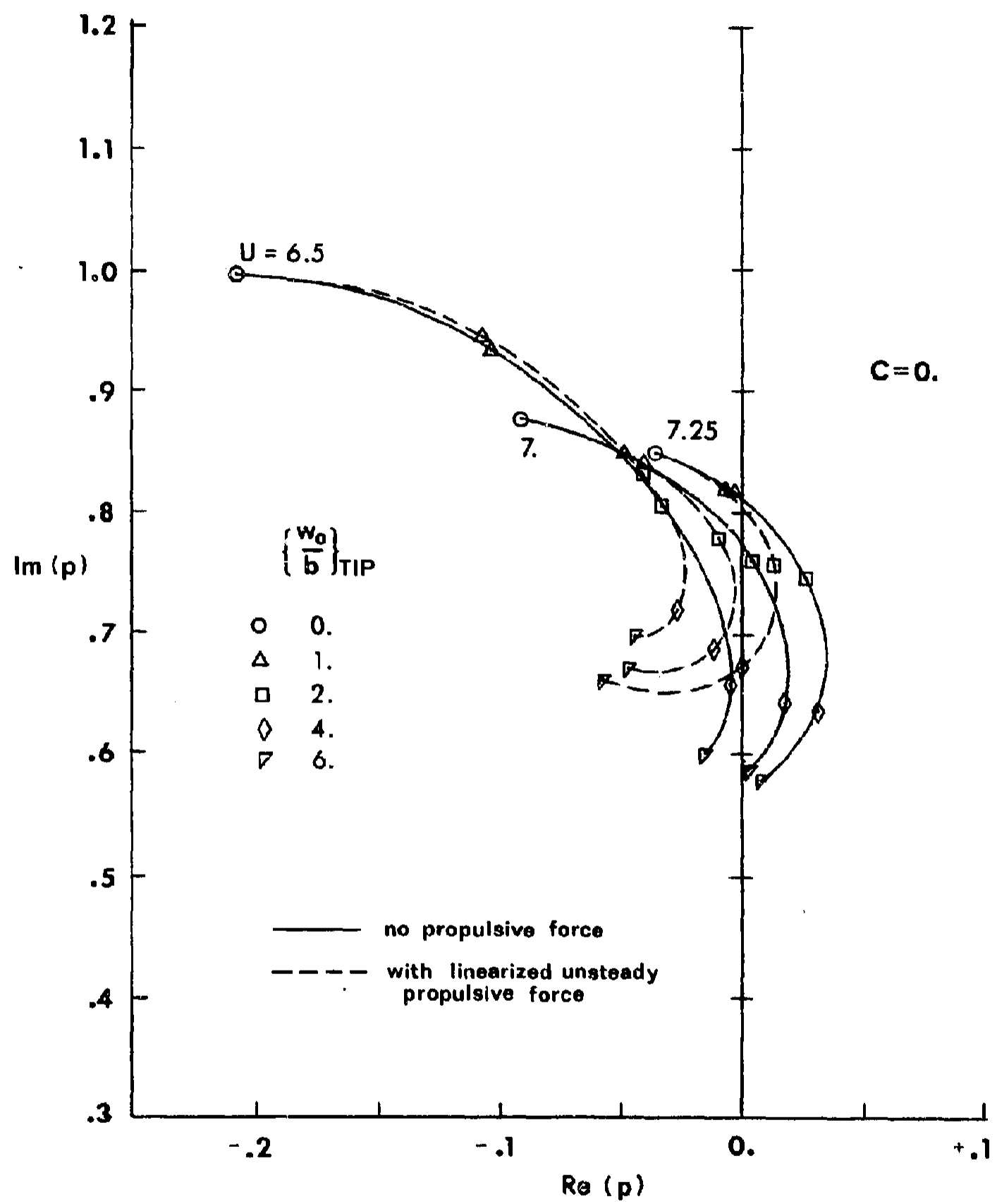

FIGURE 6-35 Effect of Including Unsteady Leading Edge Suction Forces Upon the Constant Speed Branches of Fig. 6-19 


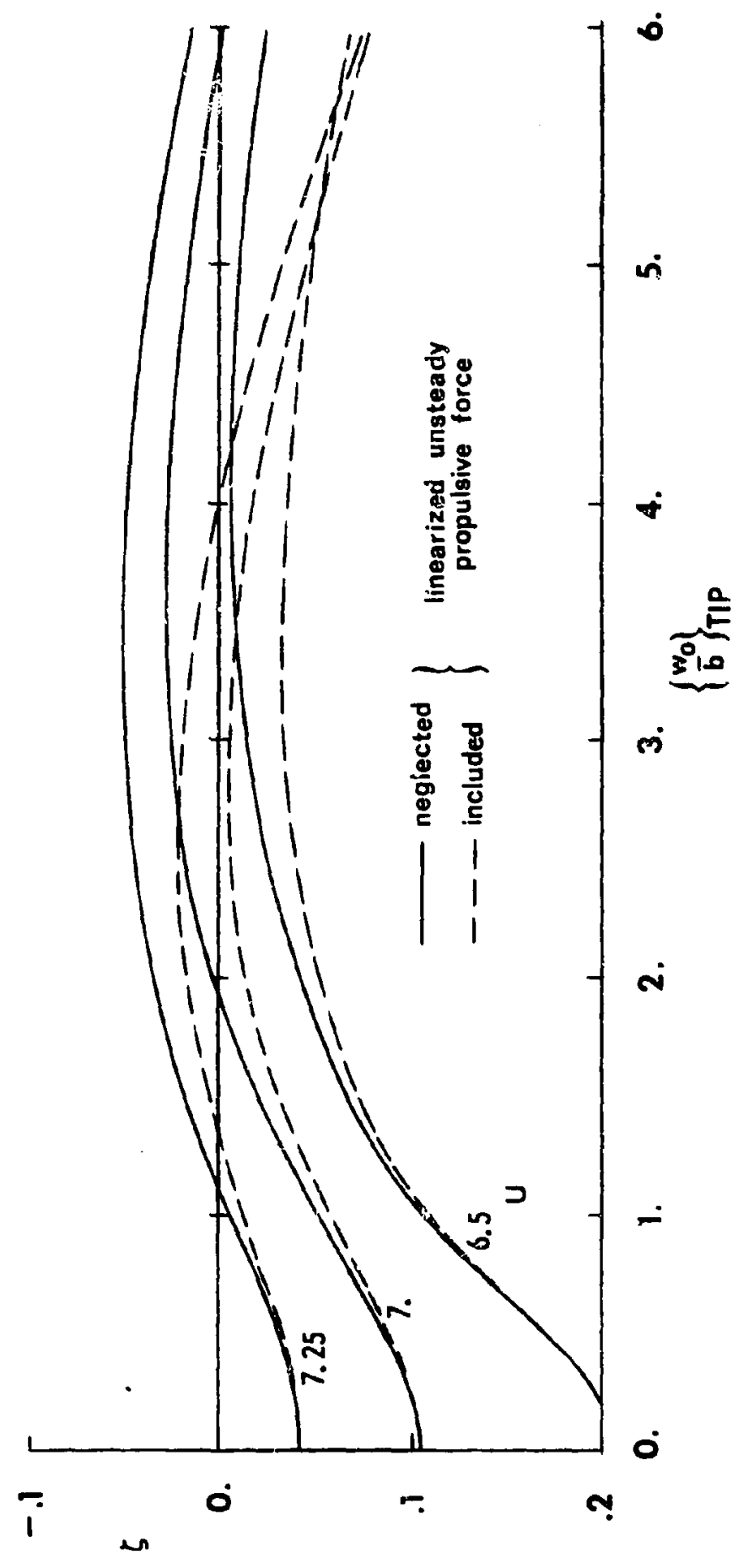

年 


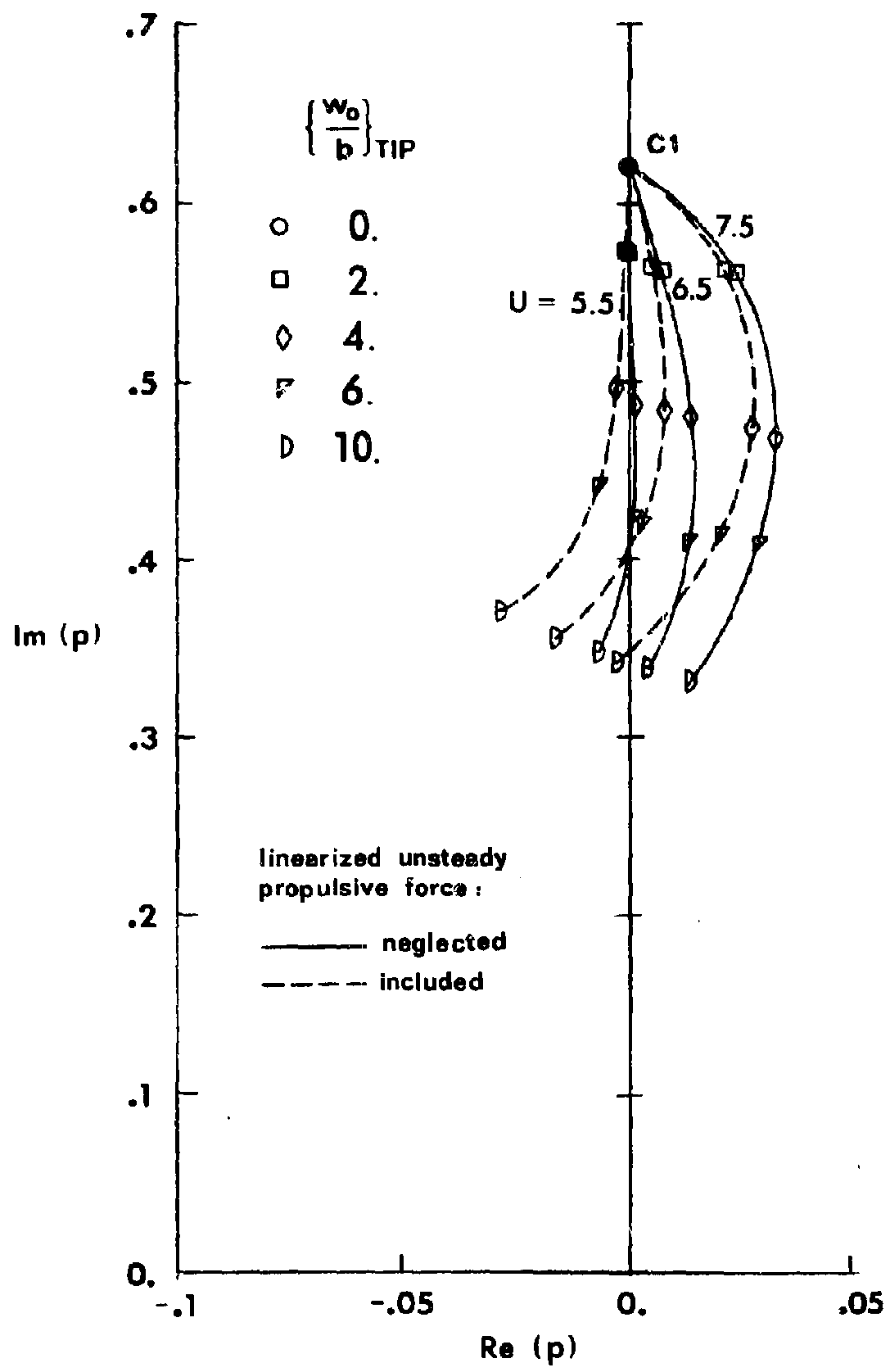

FIGURE 6-37 Effect of Allowing for Unsteady Leading Edge Suction Upon the Constant Speed Branches of F1g. 20 


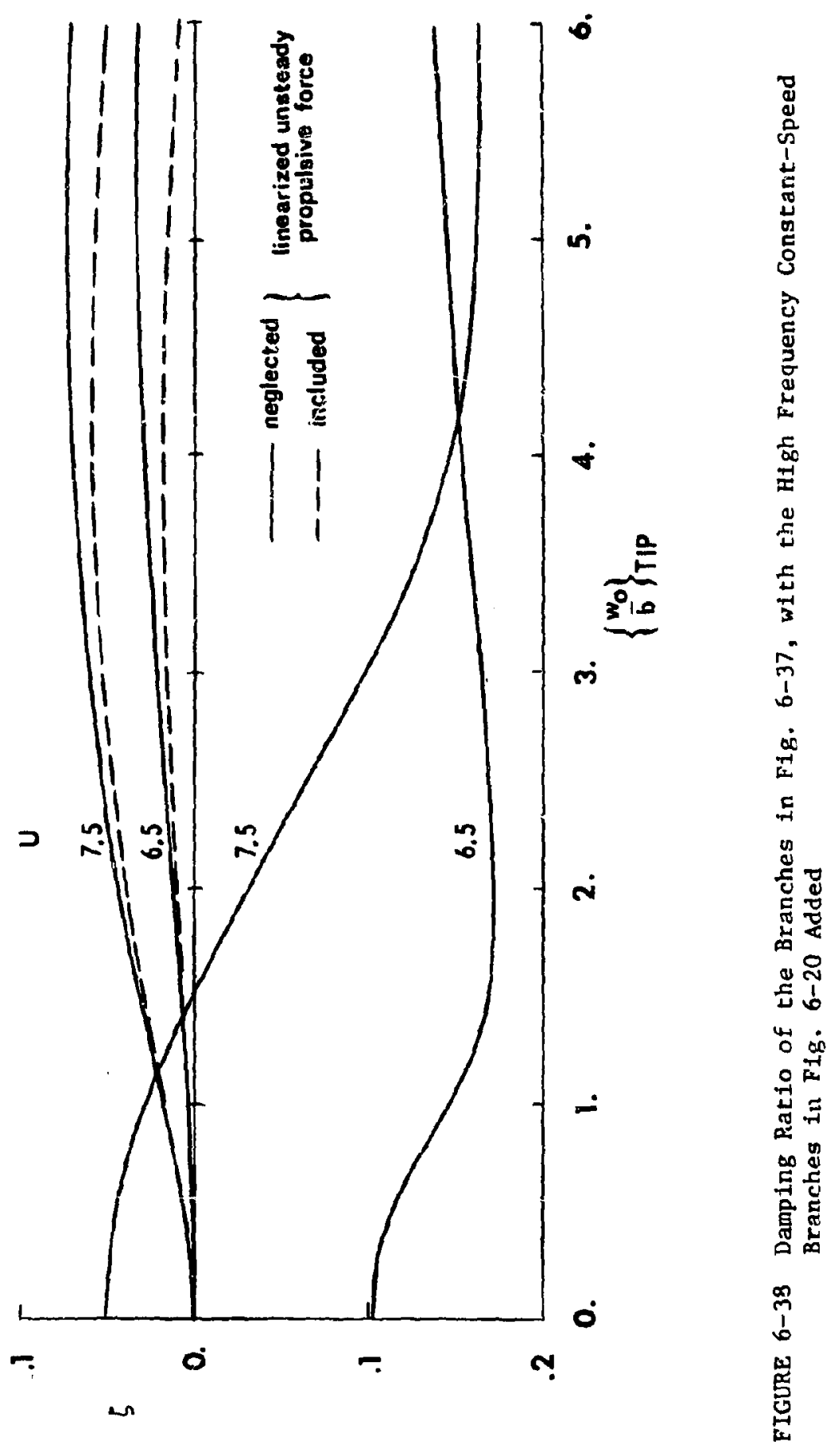




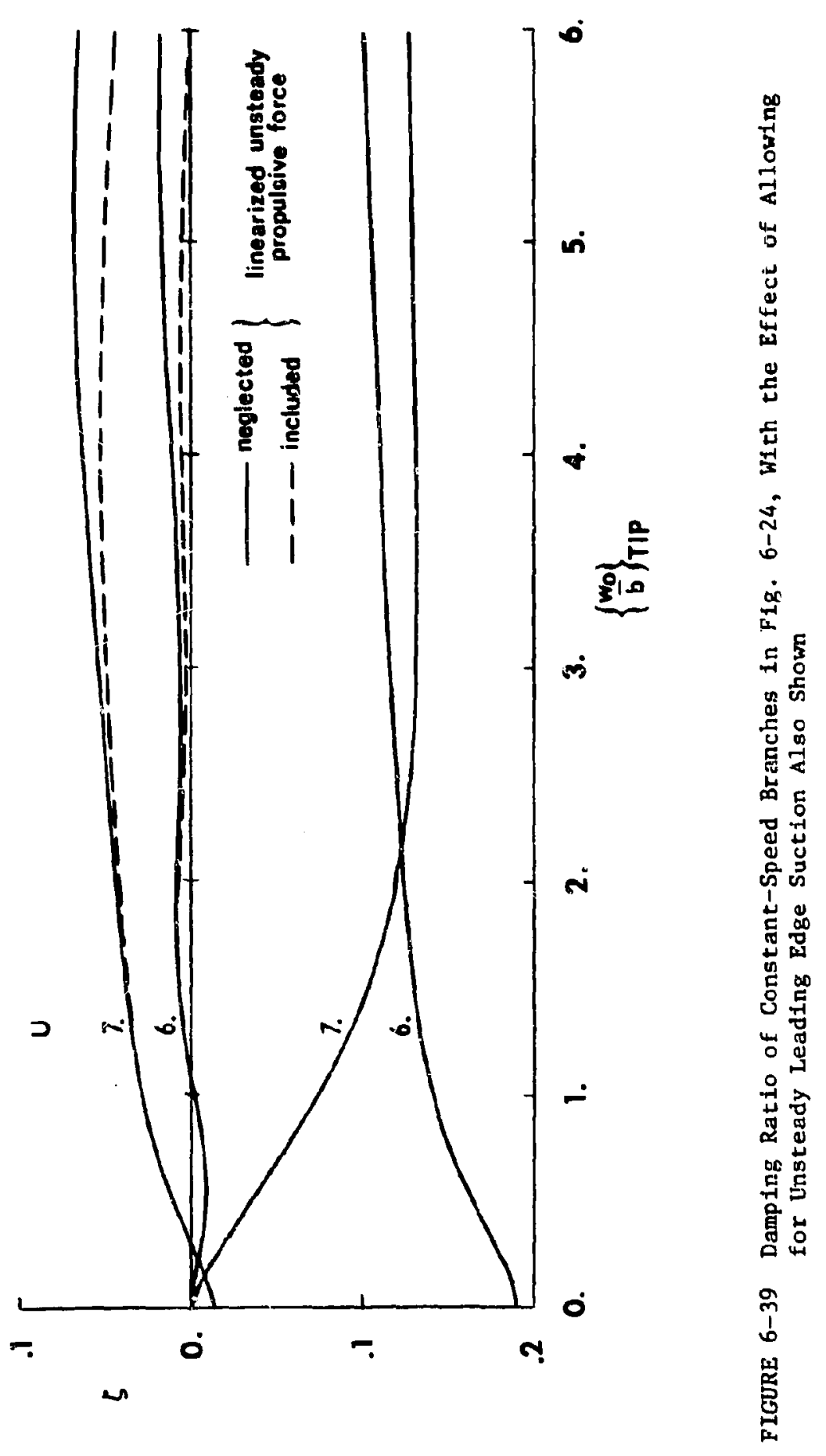




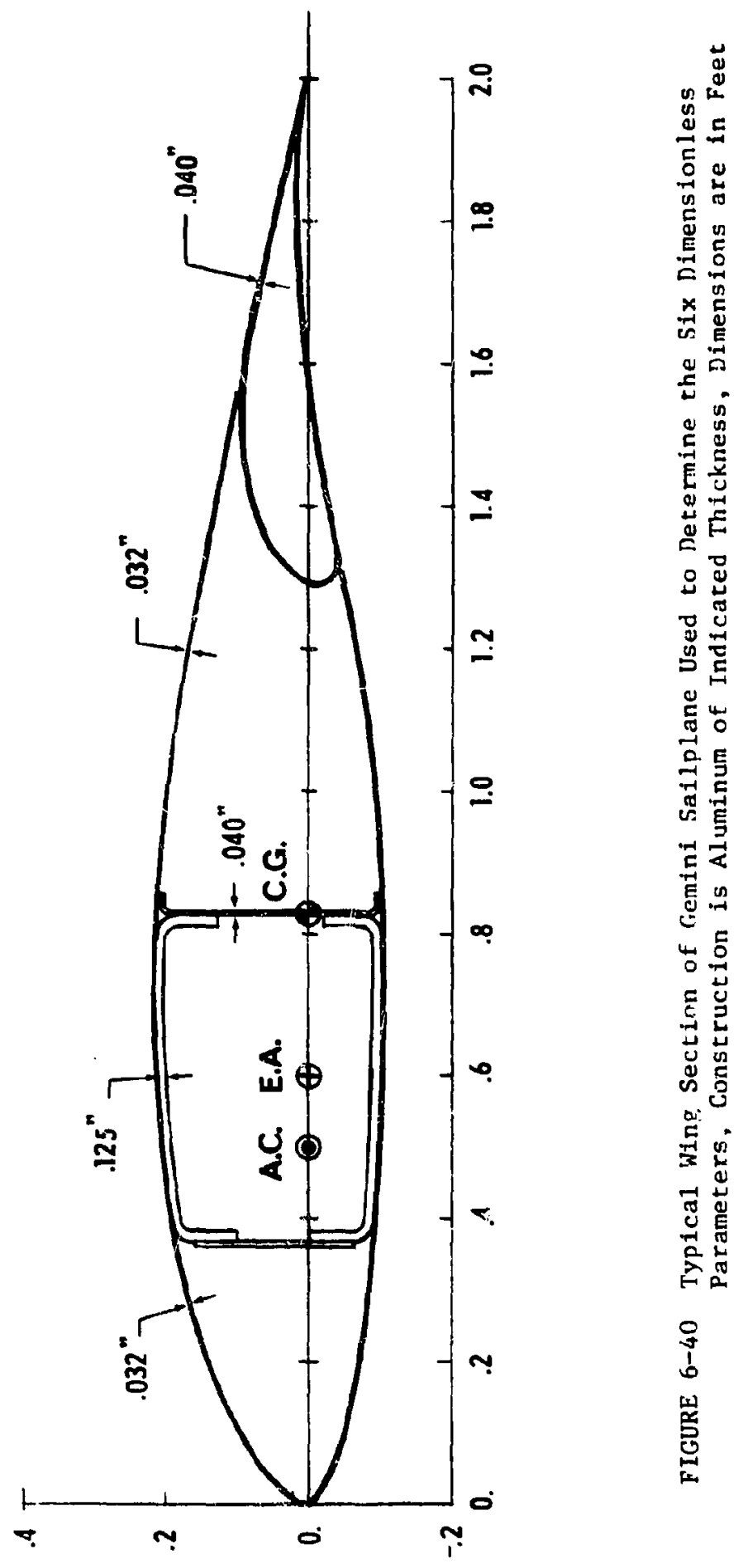

193 


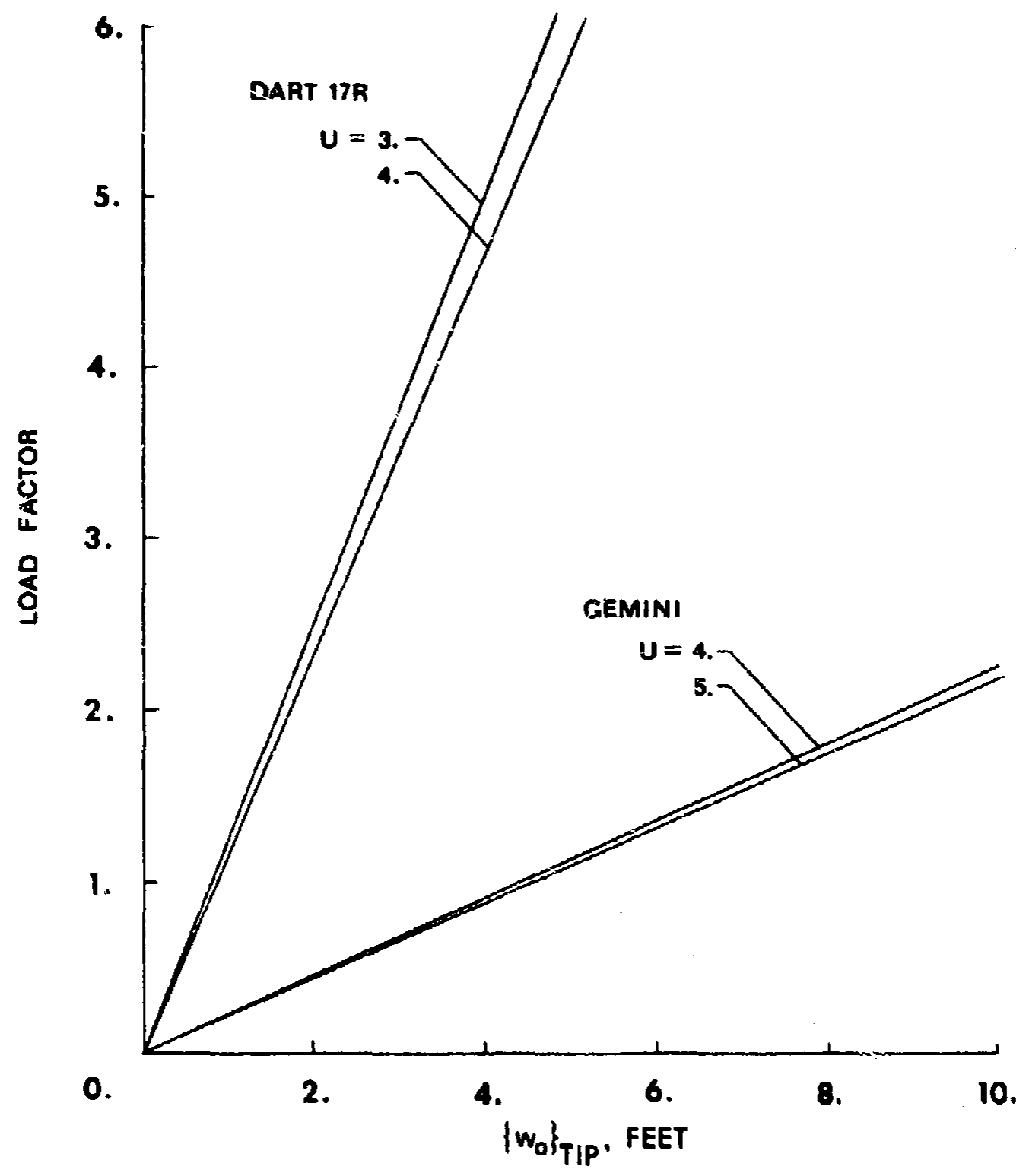

FIGURE 6-41 Comparison of Vertical Bending Deflections of the Two Sallplane Wing Models for Given Steady Load Factor 


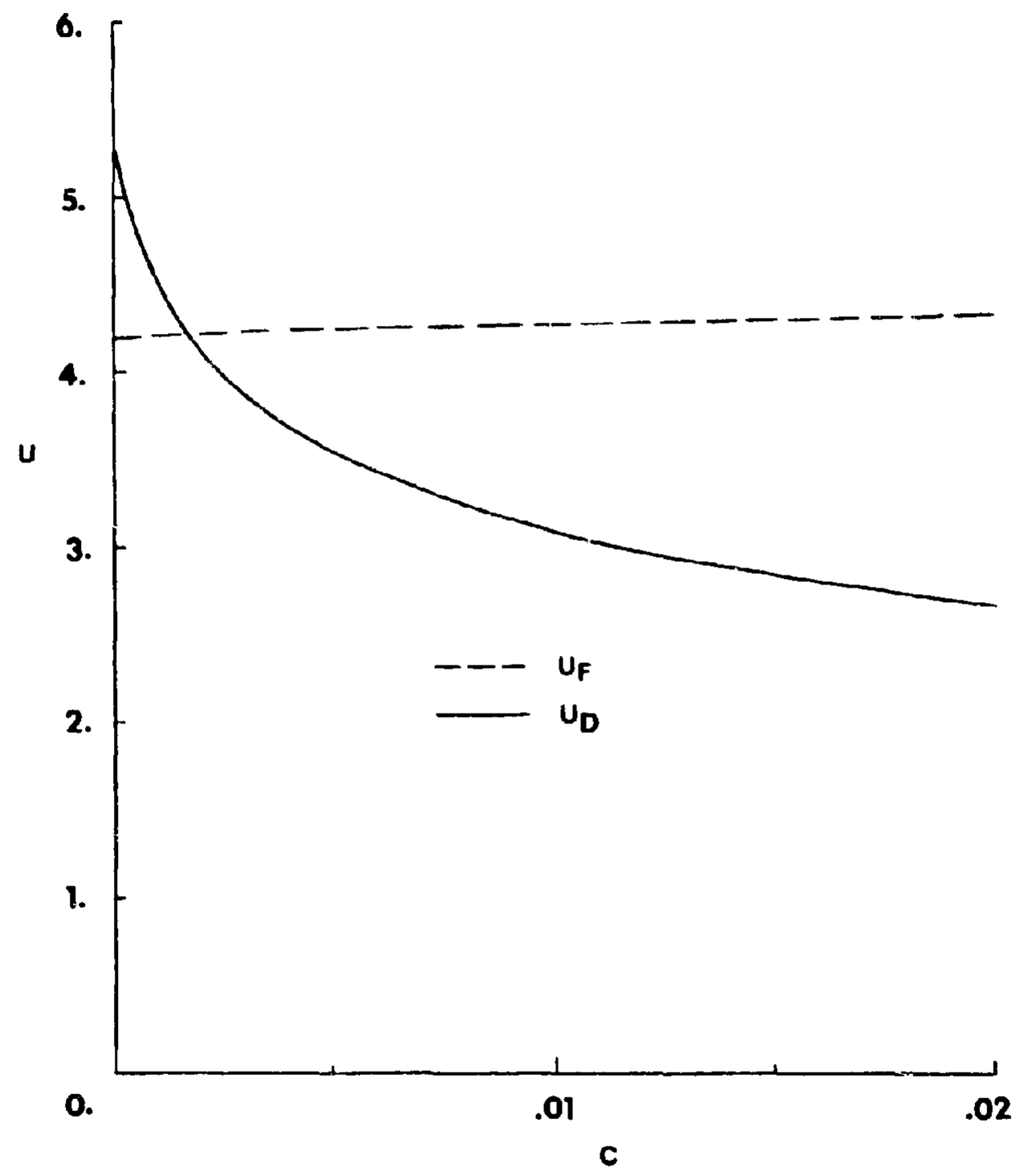

FIGURE 6-42 Effect of Steady Drag on Divergence and Zero-Lift Flutter Speeds of the Dart 17R Model 


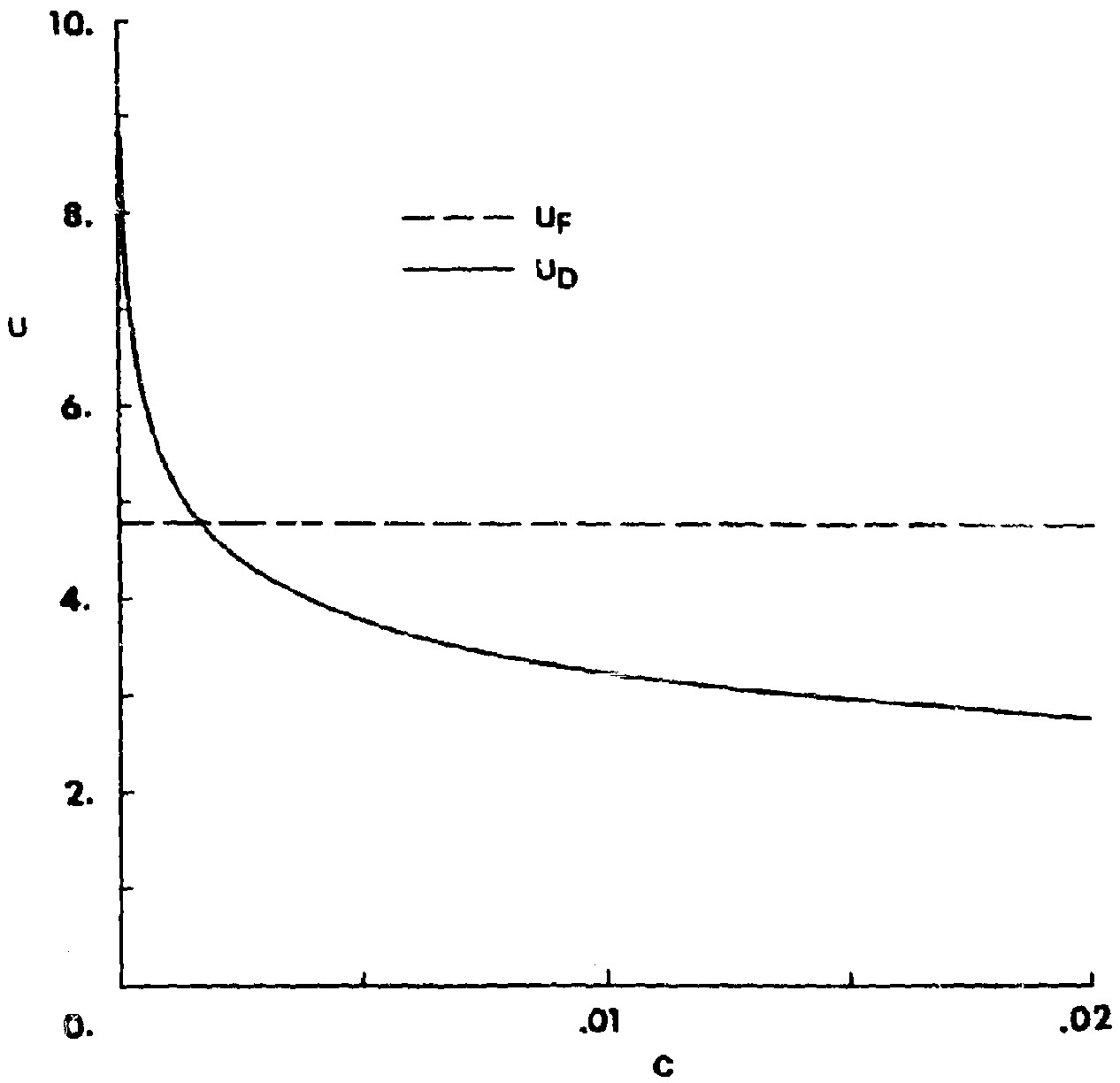

FIGURE 6-43 Effect of Steady Drag on Divergence and Zero-Lift Flutter Speeds of the Gemini Model 


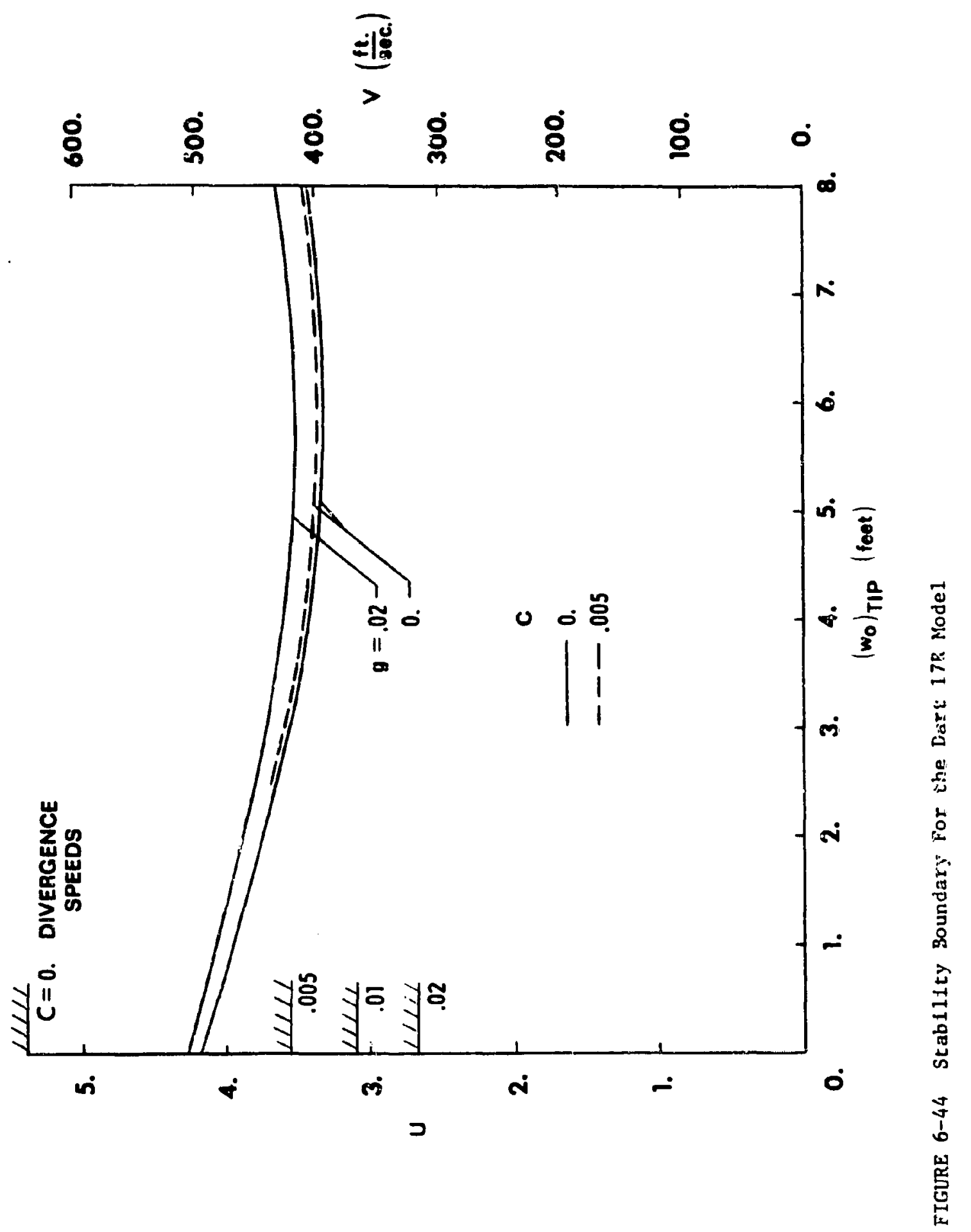




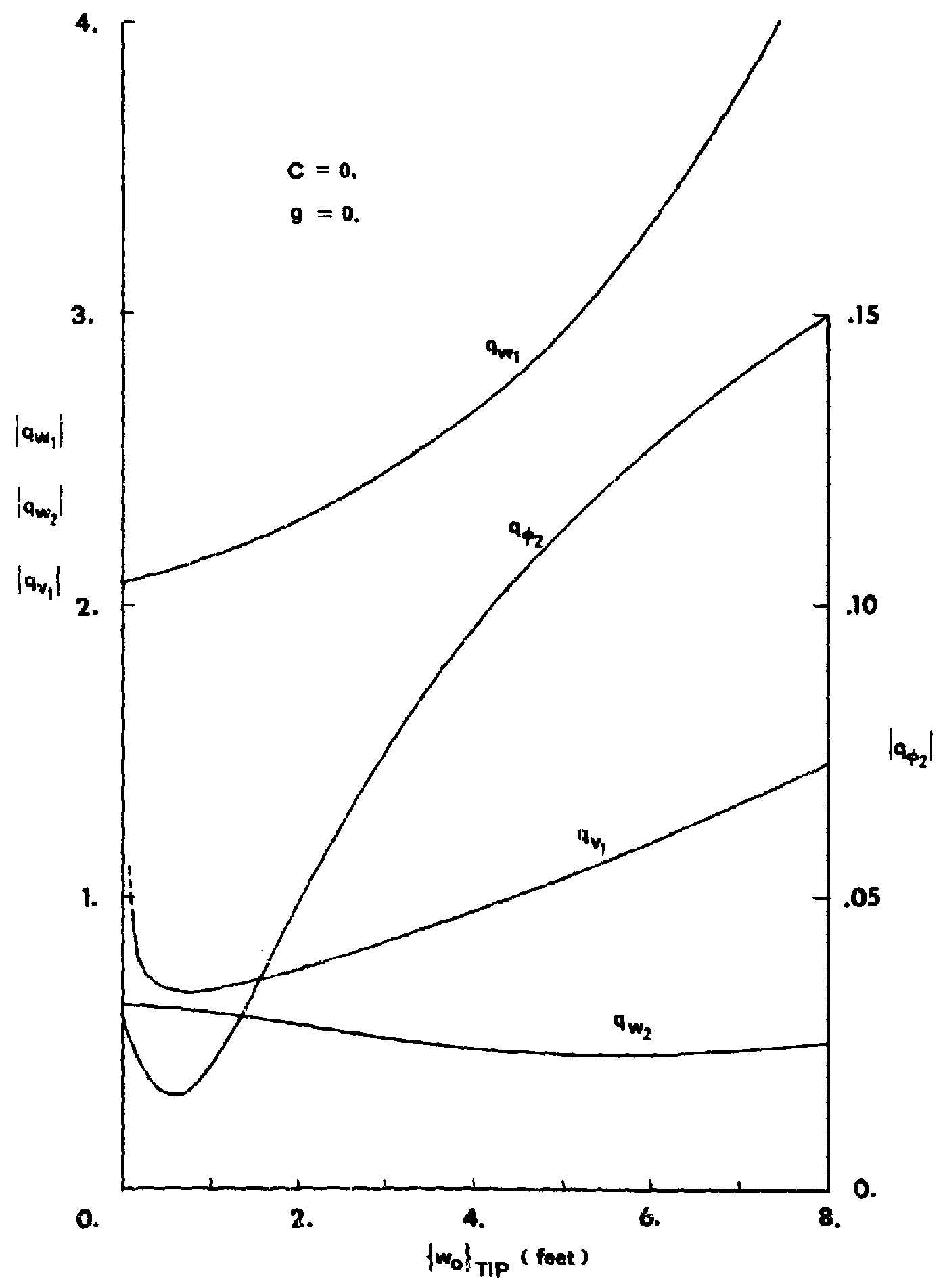

FIGURE 6-45(a) Dart 1?R Flutter Moce Shape Amplitudes, Normallzed to $\left|q_{\Phi_{1}}\right|$ 


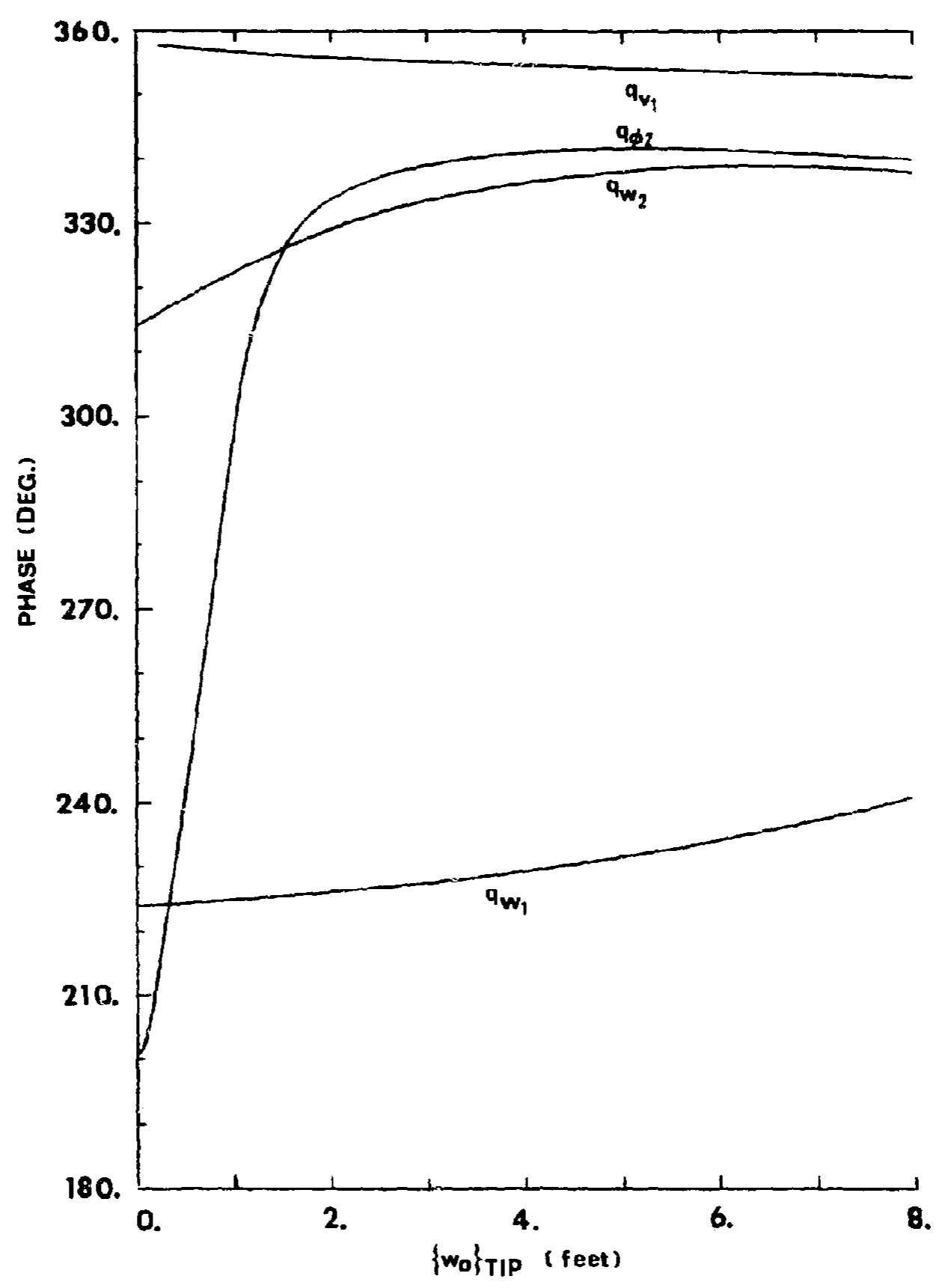

FIGURE 6-45(b) Dart 17R Flitter Mode Shape Phase Angles for Zero Phase of $q_{\phi}$ 


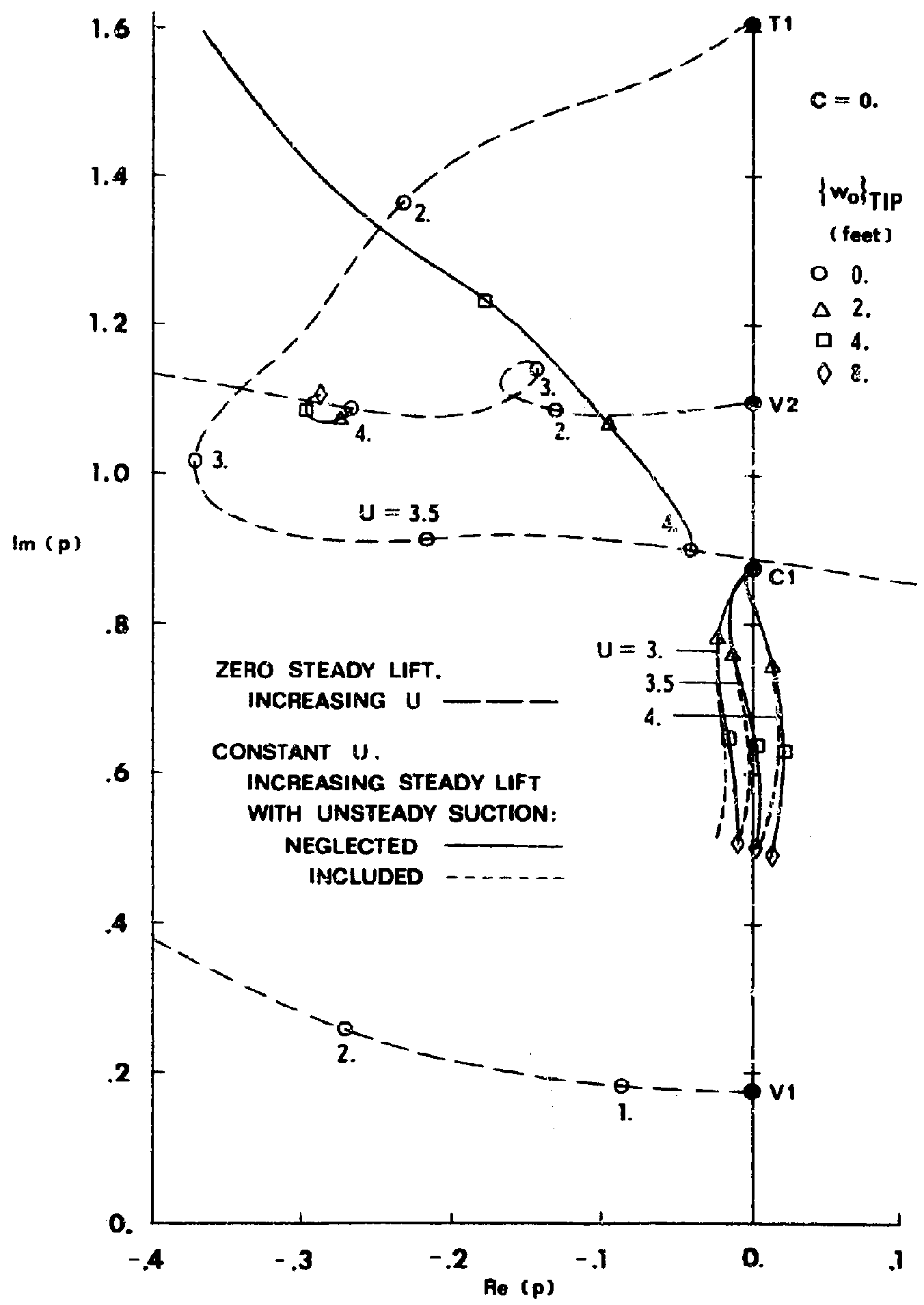

FIGURE 6-46 Locus of Roots for True Aeroelastic Modes, Dart i.7R Model 


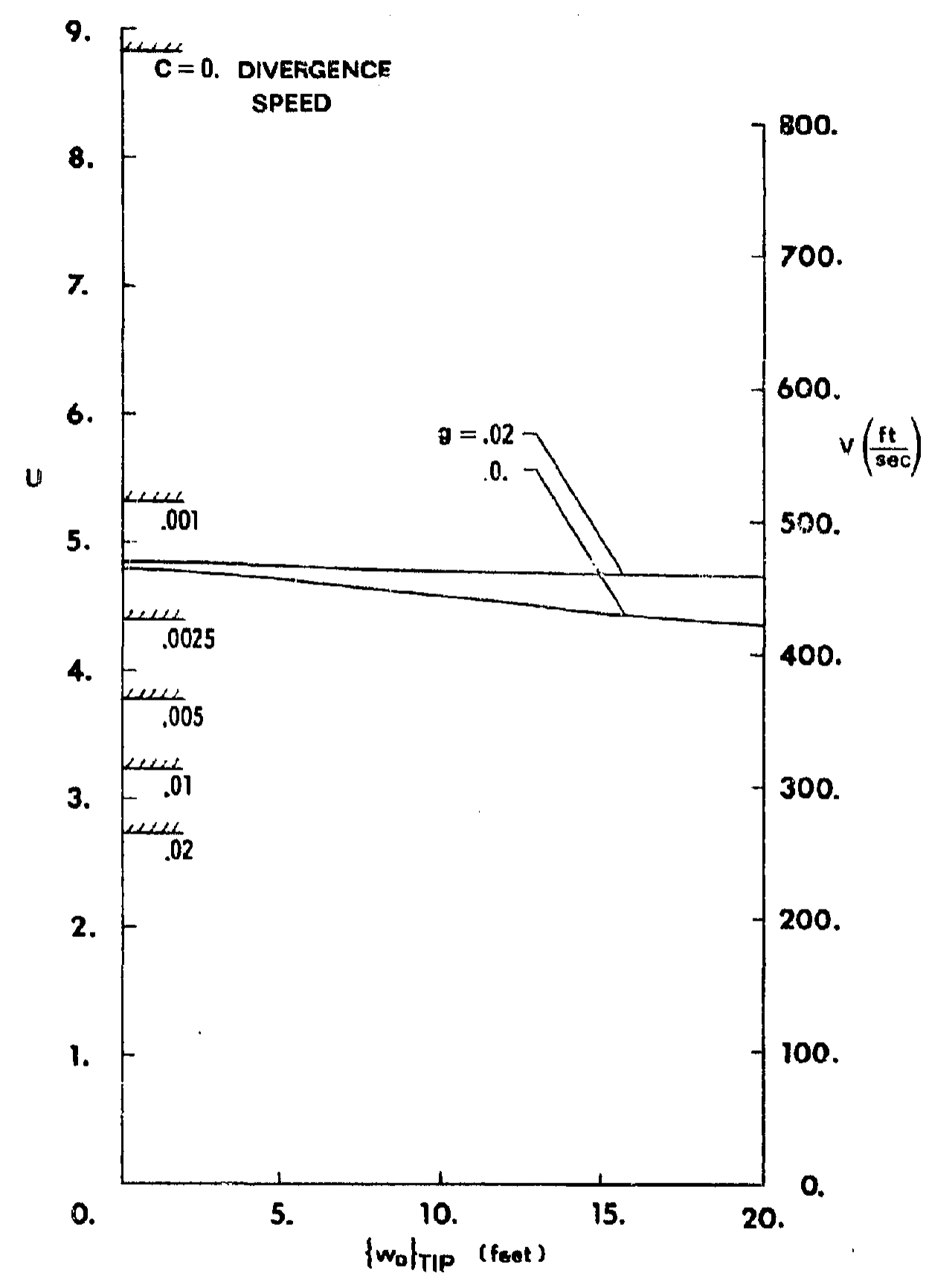

FIGliRE 6-47 Stablifty Boundary for the Geminf Mode1 

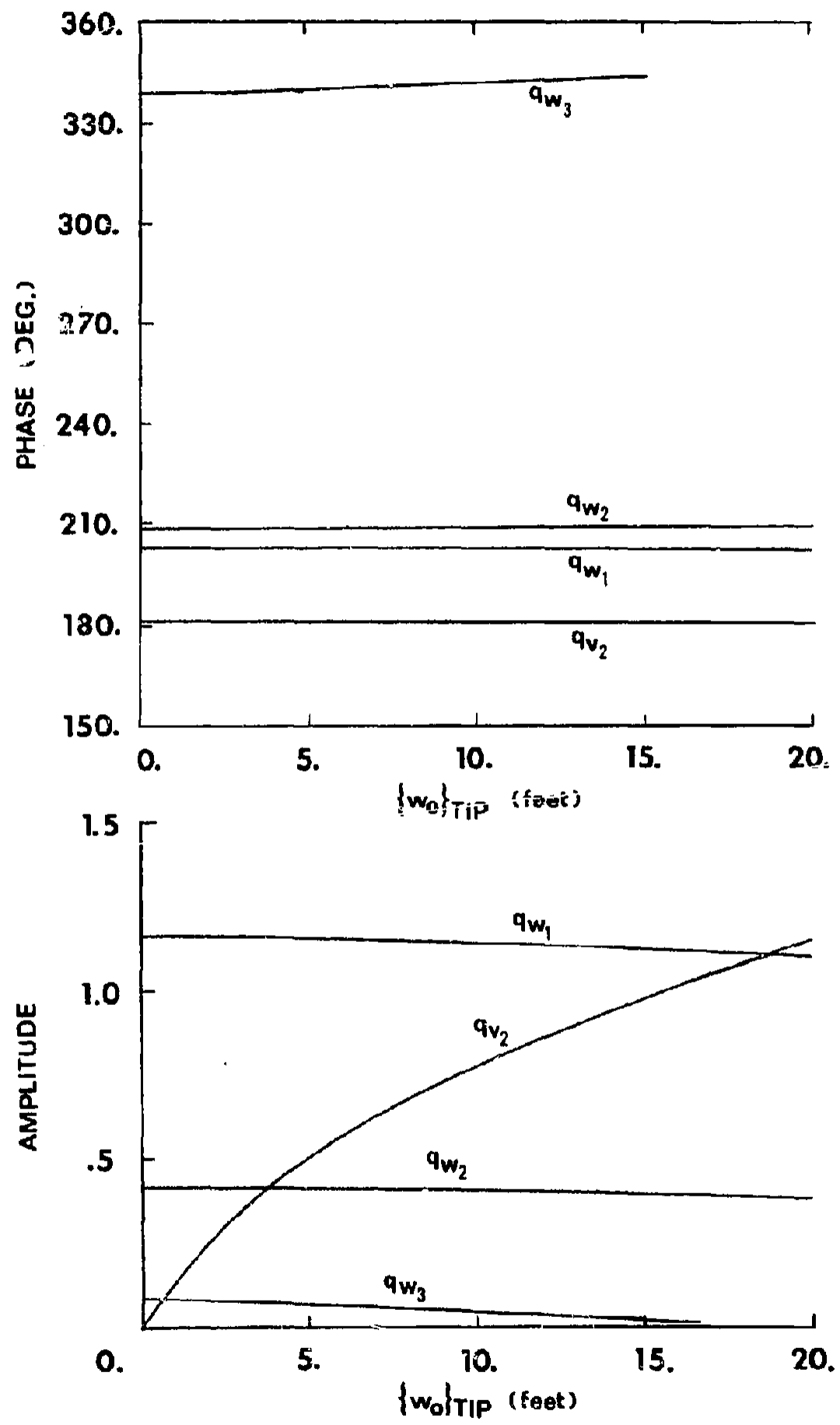

FIGURE 6-48 Flutter Mode Shape Amplitudes and Phase Angles for the Gemini Model, for Unlt Amplitude and Zero Phase of ${ }^{q_{\phi_{1}}}$ 


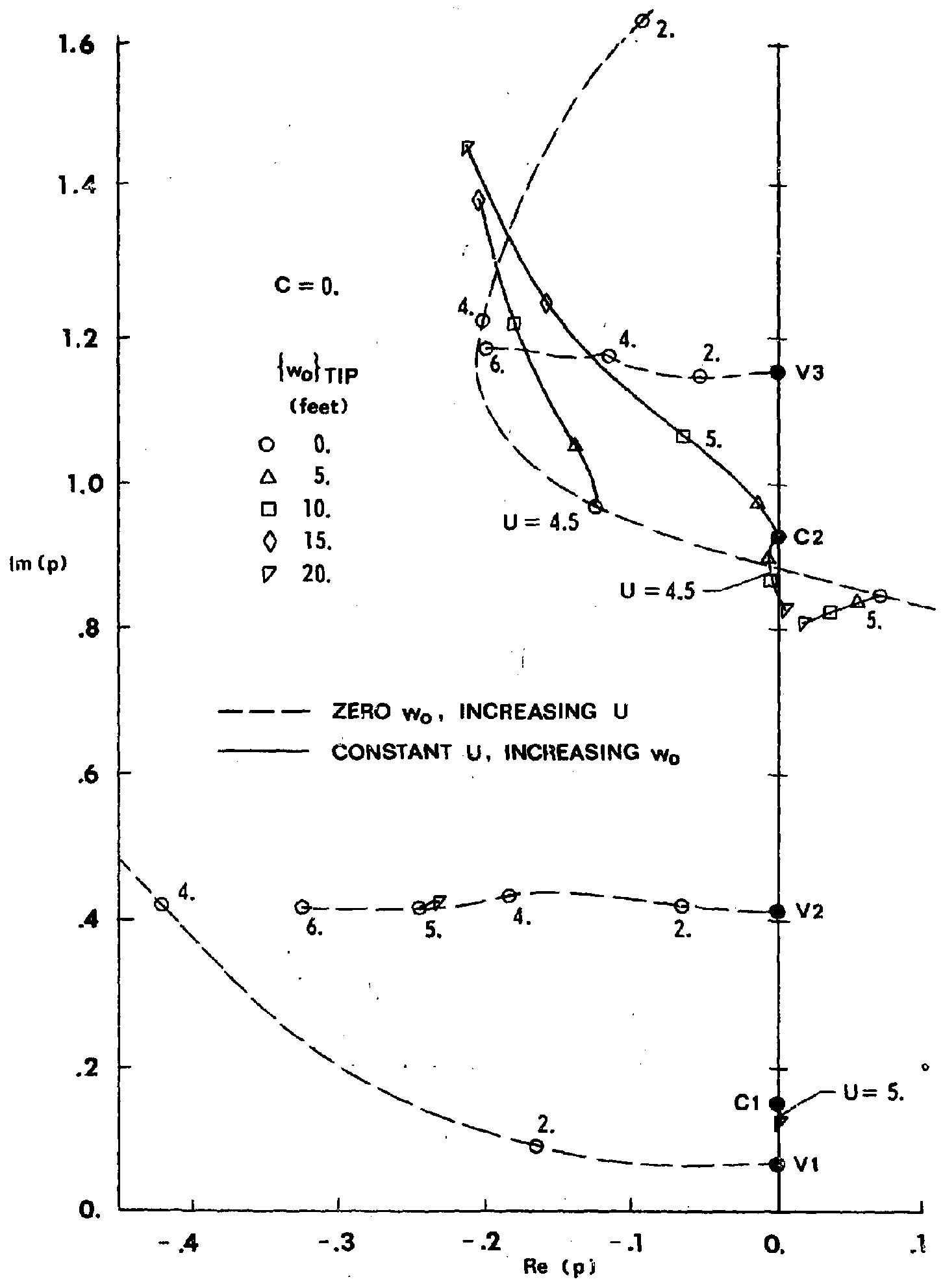

FIGURE 6-49 Locus of Roots for True faroelastic Modes, Gemini Mode1 


\section{Chapter VII}

FLUTTER VELOCITY USING ATRLOADS FROM THREE-DIMENSIONAL SUBSONIC AERODYNAMIC THEORY

\section{A. Incluston of Three-Dimensional Airloads}

The influence of steady deformations and chordwise forces upon dynamic stability of the unfform cantilever wing has been examined in Chapter VI using lifting airloads predicted by incompressible steady and unsteady strip-theory. This approximate modeling of the aerodynamic loads made possible their convenient numerical computation for any convergent, neutral, or divergent oscillations of incerest. As a result, the iterative solution schemes of Chapters IV and $V$ could be developed and a variety of wing configurations could be analyzed efficiently.

The accuracy with which the incompressible strip theory results approximate the three-dimensional compressible flow situation is next explored by extension to subsonic three-dimensional lifting airloads. The simple harmonic flutter solution method described in Chapter IV is accordingly modified to use subsonic three-dimensional steady and oscillating unsteady aerodynamic loads calculated separately by the computer program written by Rowe, et a1. (Ref. 18). Resu1ts are then found which demonstrate that the phenomena discussed in Chapter VI st111 occur after three-dimensional aerodynamics are introduced. The role of unsteady potential chordurise loads in flutter is investigated as is the effect of compressibility. Since the use of externally computed air loads requires a mnre cumbersome solution method, only enough results are sought to provide direct comparison with the Incompressible strip-theory calculations.

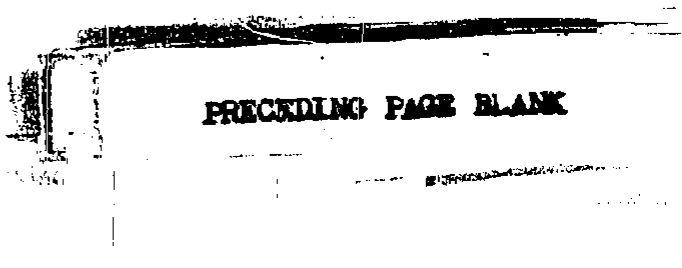


The Rowe computer program solves the pressure-downwash integral equation (Ref. 24) for compressible flow about a steady or osclilating planform. The kinematic downwash boundary condition for each structural mode is enforced by collocation, at a set of user-specified points, of the downwash distributions associated with an assumed series of pressure functions. For this application seven collocation chords are specified having five collocation polnts per chord. Six elastic structural modes are input-- three in vertical banding and three in torsion. The three torsion modes are defined for $A=.1$ (lateral displacements depend on elastic axis location). A thorough description of the theoretical aspects of the subsonic kernel function program is provided in Ref. 18, and the programming detalls are documented in Ref. 19. Its capability for modeling trailing- and leading-edge control surfaces is not required in the present application.

The unsteady potential chordurise forces can be deduced from program output, as follows. For each structural. mode and its downwash the program caiculates the complex amplitucie of an associated distributed lifting pressure difference on the rectangular planform, which in dimensionless form for the $f$ th mode is, per unit $q_{j}$ having steady or simple harmonic time dependence,

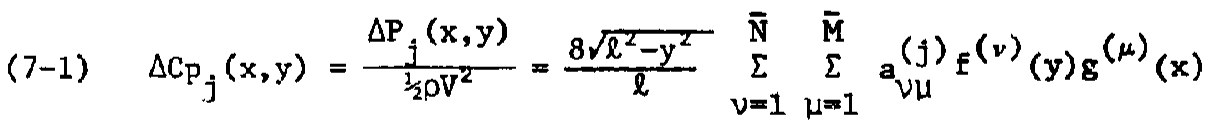
Here $a_{V \mu}^{(j)}$ are coefficient multipliers of the series expansion of pressure on the planform, which can be 11sted in the program output. The assumed spanwise pressure distributions are 


$$
\text { (7-2) } \quad \begin{aligned}
f^{(v)}(y) & =\frac{\sin (2 v-1) \Phi}{\sin \Phi}, v=1,2,3, \ldots \bar{N} \\
(7-3) \quad \Phi & =\cos ^{-1}\left(\frac{y}{\ell}\right) \\
\bar{N} & =\text { number of downwash chords on semispan }
\end{aligned}
$$

The assumed chordwise pressure distributions, dependent upon $x$ only for a rectangular planform, are

$$
\begin{aligned}
& \text { (7-4) } \mathrm{g}^{(\mu)}(\mathrm{x})= \begin{cases}\cot \frac{\theta}{2} & \mu=1 \\
\sin (\mu-1) \theta & \mu=2,3,4, \ldots \overline{\mathrm{M}}\end{cases} \\
&(7-5) \quad \theta=\cos ^{-1}\left(-\frac{\mathrm{x}}{\mathrm{b}}\right) \\
& \overline{\mathrm{M}}=\text { number of downwash points on a downwash chord }
\end{aligned}
$$

where $x$ is measured aft from the midchord.

The resultant chordwise component of potential airloads, acting in the positive $x$ direction, can be expressed as

$$
\text { (7-6) } \quad D_{p}(y ; t)=-z_{s} p V^{2} \int_{-b}^{b} \Delta C_{p}(x, y ; t) \frac{\partial z s}{\partial x}(x, y ; t) d x-F_{s}(y ; t)
$$

The first term represents the $x$-component of the force, which is normal to the defiected chord, second term contains the contribution of ieadingedge suction. It is an idealization of Inearized theory, which is supposed to approximate the actual effects of low pressure acting around a curved leading edge.

Steady and unsteady parts of the pressure and mean-surface chordwise slope can be separated: 
$(7-7)$

$$
\Delta C_{p}(x, y ; t)=\Delta C_{P_{0}}(x, y)+\Delta C_{p_{1}}(x, y ; t)
$$

(7-8) $\quad \frac{\partial z}{\partial x}(x, y ; t)=-\phi_{0}(y)-\phi_{1}(y ; t)$

The first term of (7-6) will then contain a steady part, a linear unsteady part, and a nonlinear part, as follows:

$$
\begin{aligned}
D_{p}(y ; t)= & \frac{1}{2} \rho V^{2} \int_{-b}^{b} \Delta C_{p_{0}} \phi_{0} d x \\
& +1 \frac{1}{s} \rho V^{2} \int_{-b}^{b}\left(\Delta C_{p_{0}} \phi_{1}+\Delta C_{p_{1}} \phi_{o}\right) d x \\
& +1_{2} \rho V^{2} \int_{-b}^{b} \Delta C_{p_{1}} \phi_{1} d x-F_{s}(y, t)
\end{aligned}
$$

The suction force likewise can be separated. The leading-edge inversesquare-root pressure singularity strengths for steady and unsteady flow can be defined,

$(7-10) \quad C_{F_{0}}(y) \equiv \frac{V}{4} \underset{x \rightarrow-b}{\lim }\left[-\sqrt{x+b} \Delta C_{p_{0}}(x, y)\right]$

(7-11) $\quad \delta_{F_{i}}(y ; t) \equiv \frac{V}{4} \underset{x \rightarrow-b}{\lim }\left[-\sqrt{x+b} \Delta c_{p_{1}}(x, y ; t)\right]$

From equations $(5-9)$ and $(5-10)$, the leading edge suction force in terms of the vorticity singularity is

$$
F_{s}(y ; t)=\sqrt{1-M_{a}^{2}} \frac{\pi}{4} o[\underset{x \rightarrow-b}{11 m}(\sqrt{x+b} \gamma(x, y ; t))]^{2}
$$

where the effect of compressibility is now included with the $\sqrt{1-\mathrm{N}^{2}}$ factor (deduced from Eq. 12-1 of Ref, 26). Vorticity and pressure discontinuity distributions in the vicinity of the leading edge are related 
by (Ref. 23, Eq. (5-93))

$(7-13) \quad \Delta C_{p}(x, y ; t)=-\frac{2 \gamma}{V}(x, y ; t)$

The suction force in terms of the leading edge pressure singularity is, therefore,

$$
\text { (7-14) } \begin{aligned}
F_{s}(y ; t) & =\sqrt{1-M_{a}^{2}} \Pi \rho\left\{\frac{V}{4} \underset{x+b}{\lim }\left[-\sqrt{x+b} \Delta C_{p}(x, y ; t)\right]\right\}^{2} \\
& =\sqrt{1-M_{a}^{2}} \Pi \rho\left\{C_{F_{0}}(y)+C_{F_{1}}(y ; t)\right\}^{2} \\
& =\sqrt{1-M_{a}^{2}} \Pi \rho\left[C_{F_{o}}^{2}+2 C_{F_{o}} C_{F_{1}}+C_{F_{1}}^{2}\right]
\end{aligned}
$$

where $(7-7),(7-10)$, and $(7-11)$ have been used. Equations (7-9) and (7-14) suggest that chordwise forces do affect both the steady d1splacement solution and the linearized unsteady stability problem.

Actual computation of the suction force contributions can be uccomplished through program output of the series coefficients $a_{\nu \mu}^{(j)}$. Insertion of (7-1) into $(7-10)$ and $(7-11)$, for the $j$ th mode, leads to the steady and unsteady leading-edge singularity strerigths

$$
\begin{array}{ll}
\text { (7-15) } & c_{F_{0}^{(j)}}^{(y)}=-2 v\left[\frac{\sqrt{l^{2}-y^{2}}}{\ell} \sum_{v=1}^{\bar{N}} a_{V 1}^{(j)} f^{(v)}(y)\right] \sqrt{b} \\
(7-16) & c_{F_{1}}^{(j)}(y)=-2 v\left[\frac{\sqrt{\lambda^{2}-y^{2}}}{\ell} \sum_{v=1}^{\bar{N}} a_{V 1}^{(j)} f^{(v)}(y)\right] \sqrt{b}
\end{array}
$$

Here, In taking the limit $x+-b$, only the $\mu=1$ chordwise pressure distribution terms from (7-4) remaln since 
$(7-17) \quad \lim _{x+b}\left[\sqrt{x+b} g^{(\mu)}(x)\right]=\left\{\begin{array}{rr}\sqrt{b} & \mu=1 \\ 0 & \mu>1\end{array}\right.$

The single summations that define the modal spanwise leading-edge

pressure singularity strength can be arranged for computational purposes

(7-18) $\quad F_{j}^{f}(y)=\frac{\sqrt{\ell^{2}-y^{2}}}{\hat{l}} \sum_{v=1}^{\bar{N}} a_{v 1}^{(j)} f^{(\nu)}(y)$

(7-19) $\quad F_{j}(y)=\frac{\sqrt{l^{2}-y^{2}}}{l} \sum_{v=1}^{\bar{N}} a_{v 1}^{(j)} f^{(v)}(y)$

This notation, together with summation over all modes, allows the rotal singularity strengths (7-10) and (7-11) to be computed by

$$
C_{F_{0}}(y)=-2 V \sqrt{b} \sum_{j} F_{j}^{0}(y) q_{j}^{0}
$$

$(7-21)$

$$
C_{F_{1}}(y, t)=-2 v \sqrt{b} \sum_{j} F_{f}(y) q_{f}(t)
$$

Insertion of $(7-20)$ and $(7-21)$ into (7-14) gives for the suction force

$$
\begin{aligned}
F_{s}(y, t)= & 4 \pi \rho v^{2} b \sqrt{1-M_{a}^{2}}\left[\underset{v}{\left[\sum F_{v}^{0}(y) q_{v}^{0}\right]^{2}}\right. \\
& +2 \sum_{v j} \sum_{v} F_{v}^{0}(y) q_{v}^{0} q_{j} e^{1 \omega t} F_{j}(y) \\
& \left.\left.+\underset{j}{[} F_{j}(y) q_{j} e^{i \omega t}\right]^{2}\right\}
\end{aligned}
$$

The notation of (7-18) and (7-19) is next adjusted to 1dentify the speciflc structural modes involved. For the steady problem a Iigid pitching mode must be used to solve for pressures and loads due to 
airplane angle of attack, and tts associated singularity strength parameter is denoted as $F_{\alpha}^{0}$. For the $j$ th elastic torsion mode $F_{\phi_{j}^{0}}^{0}$ is used; of course, the vertical bending modes introduce no steady lifting loads. Unsteady, leading-edge-singularity strength parameters for the $j$ th elastic corsion and bending wodes are specified by $F_{\phi_{f}}$ and $F_{w_{j}}$. respectively. The computational form of (7-22) Is then

$$
\begin{aligned}
& F_{s}(y ; t)=4 \pi \rho v^{2} \sqrt{1-M^{2}}\left[\left[F_{q}^{0} \alpha+\sum_{v=1}^{n} F_{\phi_{v}}^{0} q_{\phi_{v}}^{0}\right]^{2}\right. \\
& +2 \sum_{j=1}^{n}\left[F_{\alpha}^{0} \alpha+\sum_{v=1}^{n} F_{\phi_{v}}^{0} q_{\phi_{v}}^{0}\right] F_{w_{j}} \frac{{ }_{w}}{b} e^{i \omega t} \\
& +2 \sum_{j=1}^{n}\left[F_{\alpha}^{0} \alpha+\sum_{v=1}^{n} F_{\phi_{v}}^{0} q_{\phi_{v}}^{0}\right] F_{\phi_{j}} q_{\phi_{j}} e^{1 \omega t t} \\
& \left.+\left[\left(\sum_{j=1}^{n} F_{w_{j}} \frac{q_{b}}{b}+\sum_{j=1}^{n} F_{\phi_{j}} q_{\phi_{j}}\right) e^{i \omega t}\right]^{2}\right\}
\end{aligned}
$$

The $x$-component of the $\triangle C p$-force, as 1ndicated in (7-9), must also be expressed in a form which permits computation. Insertion of the modal quantities

$$
\begin{aligned}
& (7-24) \quad \phi_{0}=\sum_{v=1}^{n} f_{\phi_{v}} q_{\Phi_{v}}^{0} \\
& \text { (7-25) } \quad \Delta C_{p_{0}}=\Delta C_{p_{\alpha} \alpha}^{\circ}+\sum_{\nu=1}^{n} \Delta C_{p_{\phi}}^{\circ} q_{\phi_{\nu}}^{\circ} \\
& \text { (7-26) } \phi_{1}=\sum_{j=1}^{n} f_{\phi_{j}} q_{\phi_{j}} \\
& \text { (7-27) } \Delta \mathrm{Ep_{1 }}=\sum_{j=1}^{n} \Delta \mathrm{Cp}_{\mathrm{w}_{j}} \frac{\mathrm{q}_{j}}{\mathrm{~b}}+\sum_{j=1}^{n} \Delta \mathrm{Cp}_{\phi_{j}} \mathrm{q}_{\phi_{j}}
\end{aligned}
$$


Into (7-9) leads to

$$
\begin{aligned}
& D_{p}(y ; t)=\sum_{s} \rho V^{2}\left\{\left[\sum_{v=1}^{n} \int_{-b}^{b} \Delta C p_{\alpha}^{\circ} f_{\phi_{v}} d x \alpha q_{\phi_{V}}^{\circ}\right.\right.
\end{aligned}
$$

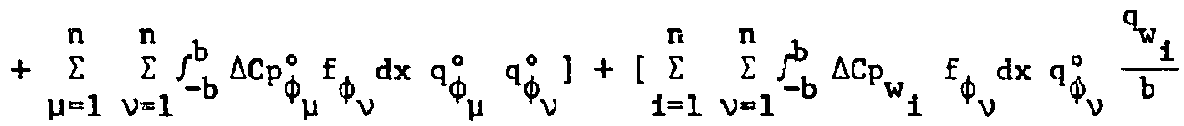

$$
\begin{aligned}
& +\sum_{i=1}^{n} \sum_{\nu=1}^{n} f_{-t}^{b} \Delta C p_{\phi_{1}} f_{\phi_{v}} d x q_{\phi_{v}}^{0} q_{\phi_{1}} \\
& +\sum_{i=1}^{n} \int_{-b}^{b} \Delta C \dot{p}_{\alpha}^{\circ} f_{\phi_{1}} d x \alpha q_{\phi_{1}}+\sum_{i=1}^{n} \sum_{\nu=1}^{n} \int_{-b}^{b} \Delta C \dot{p}_{\phi_{\nu}}^{\circ} f_{\phi_{1}} d x q_{\phi_{\nu}}^{o} q_{\phi_{1}} j e^{j \omega t} \\
& +\left[\sum_{i=1}^{n} \sum_{j=1}^{n} \int_{-b}^{b} \Delta C p_{w_{i}} f_{\phi_{j}} d x \frac{q_{w}}{b} q_{\phi_{j}}\right. \\
& \left.+\sum_{i=1}^{n} \sum_{j=1}^{n} \int_{-b}^{b} \Delta C_{\phi_{1}} f_{\phi_{j}} d x q_{\phi_{1}} q_{\phi_{j}}\right] e^{2 i \omega t}-F_{s}(y ; t)
\end{aligned}
$$

where steady, linear unsteady, and nonlinear unsteady terms in the generalized displacements are grouped together.

The chordwise integrals in (7-28) Involving the steady and unsteady modal pressures can be computed at any spanwise station by taking advantage of the Rowe program's capability to compute sectional generali.zed forces. Program output is of the form

$$
q^{s}(y)=\int_{-b}^{b} \Delta C p_{j}(x, y) H_{i}(x, y) d x
$$

where the deflection mode shape $H_{1}(x, y)$ for all integrals in $(7-28)$ will be $f_{\phi_{1}}(y)$.

The nonlisear modal equations (4-9) for steady displacements must be modified by substitution of the steady compressible lifting airloads in place of the strip theory loads (4-3). Incluston of the steady 
parts of (7-28) and (7-23) w111 account for the induced drag caused by the three-dimensionality of the flow. Generalized forces which will appear in equations $(4-9 a)$ and $(4-9 c)$ are to be computed directly, but the generalized forces for the chordwise bending equations (4-9b) involve the chordwise forces described above and must be calculated separately. These preliminary calculations require the pressure coefficients $a_{v \mu}^{(j)}$ and sectional generalized forces $0_{i j}^{s}$ obtained from Rowe program output. Program output of generalized forces is of the form $(7-30) \quad Q_{i j}=\int_{0}^{l} \int_{-b}^{b} \Delta C_{j}(x, y) H_{i}(x, y) d x d y$ where $H_{i}(x, y)$ is the ith modal displacement and $\Delta \mathrm{Cp}_{j}(x, y)$ the pressure diseriburion per unit $q_{j}$.

Rederivation of $(4-9)$ with three-dimensional subsonic steadv airloads produces the following nonlinear system:

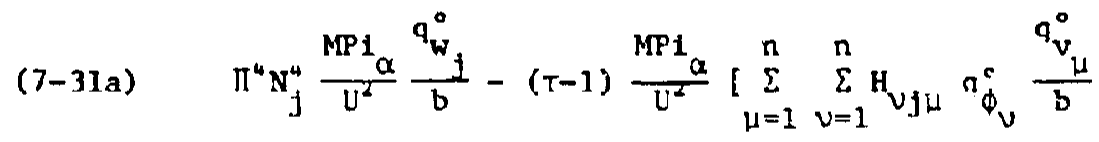

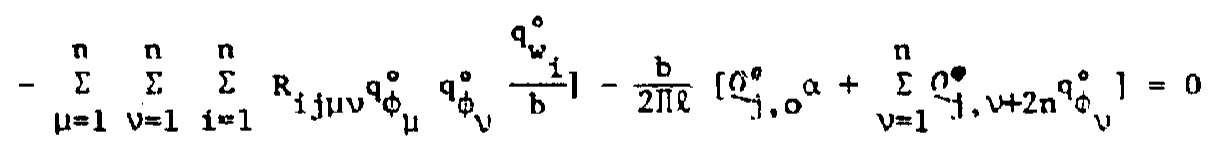


(7-31t) $\quad \pi^{4} N_{j}^{4} \frac{\tau M P I_{\alpha}}{U^{2}} \frac{q_{v}^{0}}{b}-(\tau-1) \frac{M^{P} I_{\alpha}}{U^{2}}\left[\sum_{v=1}^{n} \sum_{\mu=1}^{n} k_{v \mu j} q_{\Phi_{\nu}}^{0} \frac{q_{v}^{0}}{b}\right.$

$$
\begin{aligned}
& \left.+\sum_{\nu=1}^{n} \sum_{\mu=1}^{n} \sum_{i=1}^{n} R_{1 j \mu \nu} q_{\phi_{\nu}}^{0} q_{\phi_{L}}^{0} \frac{q_{v_{1}}^{0}}{b}\right] \\
& -\frac{1}{2 \pi l} \sum_{v=1}^{n} \int_{0}^{l}\left[f_{-b}^{b} \Delta C p_{\alpha}^{0} d x\right] f_{\phi_{v}}(y) f_{v_{f}}(y) d y q_{\phi_{v}}^{0} \alpha \\
& -\frac{I}{2 \pi l} \sum_{\mu=\ell}^{n} \sum_{\nu=1}^{n} \int_{0}^{\ell}\left[\int_{-b}^{b} \Delta C_{p_{\phi_{\mu}}}^{\circ}(x, y) d x\right] f_{\phi_{\nu}}(y) f_{v_{j}}(y) d y q_{\phi_{\mu}}^{\circ} q_{\phi_{v}}^{\circ}
\end{aligned}
$$

$(1 \leq f \leq n)$

$$
\begin{aligned}
& +4 \sqrt{1-M^{2}}\left\{\int_{0}^{l}\left(F_{\alpha}^{v}\right)^{2} f_{v} d y+2 \sum_{v=1}^{n} f_{0}^{l} F_{\alpha}^{u} F_{\phi_{v}}^{0} f_{v_{j}} d y \alpha q_{\phi_{v}}^{0}\right. \\
& \left.+\sum_{\mu=1}^{n} \sum_{v=1}^{n} \int_{0}^{l} F_{\phi_{\mu}}^{0} F_{\phi_{v}}^{0} f_{v_{j}} d y q_{\phi_{\mu}}^{0} q_{\phi_{v}}^{0}\right\}=0
\end{aligned}
$$

$(731 c)$

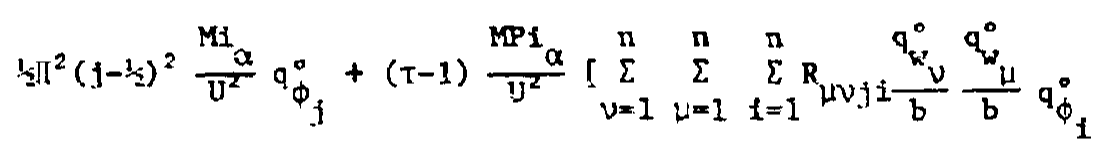

$$
\begin{aligned}
& \left.-\sum_{\nu=1}^{n} \sum_{\mu=1}^{n} \sum_{i=1}^{n} R_{\mu \nu j i} \frac{q_{v}^{0}}{b} \frac{q_{v}^{0}}{b} q_{\phi_{i}}^{0}-\sum_{\mu=1}^{n} \sum_{v=1}^{n} H_{j \nu \mu} \frac{q_{v}^{0}}{b} \frac{q^{\circ} v_{\mu}}{b}\right] \\
& -\frac{b}{2 \pi l}\left[Q_{j+2 n, 0}^{\circ} \alpha+\sum_{v=1}^{n} Q_{j+2 n, v+2 n}^{0} q_{\phi_{v}^{0}}^{0}\right]=0
\end{aligned}
$$

In (7-31) the notation $0_{i, j}^{\circ}$ refers to the steady generalized forces computed directly by the Rowe program. Here $2 n+1$ structural modes must be used to compute generalized forces: $n$ in vertical bending, $n$ in elastic torsion, and one rigid pitching mode.

Computation of the chordwise load terms in (7-31b) quickly becomes an unwieldy task as $n$ is increased, because of the profusion of numerical integrations that become necessary (for $n=3$ there would be 75 integrations). In order to avold extenstve labor but still retain 
the most significant effects of the potential chordwise forces, the following simplifying assumptions are adopted. First, lategrals appearing in the first $(j=1)$ chordwise equation of $(7-32 b)$ only are retained. Second, only the pressure distribution and slope of the first of the elastic torsion modes are kept; terms containing $\Delta \mathrm{Cp}_{\phi}^{0}$ or $\mathrm{f}_{\phi_{\mu}}$ for $\mu>1$ are dropped. Similarly, all terms containing $F_{\phi_{u}}^{\circ}$ for $\mu>1$ are removed. This approach should preserve the first-order effect of steady chordrise potential forces, yet only five numerical spanwise integrations will be required.

Actual computation of the remaining integrals

$$
\begin{aligned}
& \int_{0}^{l}\left[f_{-b}^{b} \Delta C p_{\alpha}^{0}(x, y) d x\right] f_{\phi_{1}}(y) f_{v_{1}}(y) d y \\
& j_{0}^{l}\left[\int_{-b}^{b} \Delta C p_{\phi_{1}}^{o}(x, y) d x\right] f_{\phi_{1}}(y) f_{v_{1}}(y) d y
\end{aligned}
$$

is done by direct calculation of the integrals over $x$ as sectional generalized forces with the Rowe program at eleven spanwise stations. The spanwise incegration is then carried our numerically. For the three Integrals

$$
\begin{aligned}
& \int_{0}^{l}\left[F_{\alpha}^{0}(y)\right]^{2} F_{v_{1}}(y) d y \\
& \int_{0}^{l} F_{\alpha}^{0}(y) F_{\phi_{1}^{0}}(y) f_{v_{1}}(y) d y \\
& \int_{0}^{l}\left[F_{\phi}^{0}(y)\right]^{2} f_{v_{1}}(y) d y
\end{aligned}
$$

tive sumations implied by $(7-18)$ are first made. This step is followed by spanwise numerical integration. 
The nrolinear. solution scheme described in Appendix C was straightEorwardjy adapted to compute steady deflections in subsonic threedimensional flow from (7-31). Since spanwise induction introduces coupling among the torsional modal equations, solution for linear displacements us an Initial estlmate becomes an nxn linear natrix problem. Equation (4-12) remains as the $3 n \times 3 n$ linear unsteady modal system for stabilfty about the steady equilibrium position. It now requires the generalized force matrix to be expressed for three-dimenSLomin unsteady compressible flow. As for the steady case, the generalized forces relating pressures and displacements in the vertical bending and torsion modes can be computed directly by the Rowe program, with reduced frequency and Mach number specified. Direct Insertion into $(4-12 a)$ and $(4-12 c)$ is accomplished by the simple substitucton, for generallzed forces $Q_{j, 1}$ and $Q_{f, 1}$ relating the same two modes, $(7-32) \quad Q_{j, 1}=\frac{b}{2 \pi l k^{2}} Q_{j, 1}$

Even more so than for the steady atrloads, complete Inclusion of al1 linear unsteady potential chordwise terms introduces a profusion of integrals. Formally, the linear unsteady terms which appear in (7-28) and (7-23) enter into generalized forces, computation of which involves spanwise integrations of the terms' products with $\tilde{f}_{v_{i}}$. Practically, simplifying assumptions of the type made for the steady chordwise terms have been made to keep the number of numerical integrations at a manageable level. Accordingly, only the first chordwise equation of (4-12b) will retain the chordwlse force terms. Furthermore, only the chordwise force Integrals containing pressure contributions 
and displacements for the $q_{\phi_{1}}, q_{w_{1}}$, and $q_{w_{2}}$ degrees of freedom will be kept. (Justification of this approximation is based on the flutter behavior observed in Chapter VI, which revealed little participation by the remaining vertical bending and torsion modes.) The remaining linear unsteady terms from $(7-23)$ and $(7-28)$ are

(7-33) $\quad D_{p}(y ; t) \cong \frac{1}{2} \rho v^{2}\left\{\int_{-b}^{b} \Delta C p_{w_{1}} f_{\phi_{1}} d x q_{\phi_{1}}^{0} \frac{q_{w_{1}}(t)}{b}\right.$

$$
\begin{aligned}
& +\int_{-b}^{b} \Delta C p_{w_{2}}{ }^{f} \phi_{1} d x q_{\phi_{1}}^{o} \frac{q_{w_{2}}(t)}{b}+\int_{-b}^{b} \Delta C p_{\phi_{1}} f_{\phi_{1}} d x q_{\phi_{1}}^{o}{ }^{q_{\phi_{1}}}(t) \\
& \left.+\int_{-b}^{b} \Delta C p_{\alpha}^{0} f_{\phi_{1}} d x \propto q_{\phi_{1}}(t)+\int_{-b}^{b} \Delta C p_{\phi_{1}}^{0} f_{\phi_{1}} d x q_{\phi_{1}}^{0} q_{\phi_{1}}(t)\right\} \\
& +8 \Pi \rho V^{2} \sqrt{1-M_{a}^{2}}\left\{F_{\alpha}^{0} F_{w_{1}} \alpha \frac{q_{w_{1}}(t)}{b}+F_{\alpha}^{0} F_{w_{2}} \alpha \frac{\mathrm{q}_{2}(c)}{b}\right. \\
& +F_{\alpha}^{o} F_{\phi_{1}} \alpha q_{\phi_{1}}(t)+F_{\phi_{1}}^{o} F_{w_{1}} q_{\phi_{1}}^{o}{\frac{q_{1}}{b}}^{(t)} \\
& \left.+F_{\phi_{1}}^{\circ} F_{w_{2}} q_{\phi_{1}}^{\circ} \frac{q_{w_{2}}}{b}+F_{\phi_{1}}^{\circ} F_{\phi_{1}} q_{\phi_{1}}^{\circ} q_{\phi_{1}}(t)\right\}
\end{aligned}
$$

The generalized force matrix terms for inclusion in $(4-12 b)$ are then found to be

$$
\begin{gathered}
Q_{\perp+n, 1}=\frac{b}{2 \pi k^{2} l} \int_{0}^{l}\left[\int_{-b}^{b} \Delta C{p_{w_{1}}}_{1}(x, y) d x\right] f_{\phi_{1}}(y) f_{v_{1}}(y) \text { dy } q_{\phi_{1}}^{0} \\
-\frac{8 b}{k^{2} l} \sqrt{1-M_{a}^{2}}\left\{\int_{0}^{l} F_{\alpha}^{o}(y) F_{w_{1}}(y) f_{v_{1}}(y) d y \alpha+\int_{0}^{l} F_{\phi_{1}}^{o} F_{w_{1}} f_{v_{1}} d y q_{\phi_{1}}^{o}\right\}
\end{gathered}
$$




$$
\begin{aligned}
& \begin{array}{l}
\begin{array}{l}
(7-34) \\
\text { con't }
\end{array} \quad Q_{1+n, 2}
\end{array} \quad=\frac{b}{2 \pi k^{2} l} \int_{0}^{l}\left[\int_{-b}^{b} \Delta C_{w_{2}}(x, y) d x\right] f_{\phi_{1}}(y) f_{v_{1}}(y) d y q_{\phi_{1}}^{0} \\
& -\frac{8 b}{k^{2} \ell} \sqrt{1-M_{a}^{2}}\left\{\int_{0}^{l} F_{\alpha}^{0} F_{w_{2}} f_{v_{1}} \text { dy } \alpha+\int_{0}^{l} F_{\phi_{1}}^{\circ} F_{w_{2}} f_{v_{1}} \text { dy } q_{\phi_{1}}^{0}\right\} \\
& Q_{1+n, 1+2 n}=\frac{b}{2 \pi k^{2} \ell}\left\{\int_{0}^{l} \int_{-b}^{b} \Delta C p_{\alpha}^{\circ} d x\right] f_{\phi_{1}} f_{v_{1}} d y \alpha \\
& \left.+\int_{0}^{l}\left[\int_{-b}^{b} \Delta C p_{\phi_{1}}^{\circ} d x\right] f_{\phi_{1}} f_{v_{1}} d y q_{\phi_{1}}^{\circ}+\int_{0}^{l}\left[\int_{-b}^{b} \Delta C p_{\phi_{1}} d x\right] f_{\phi_{1}} f_{v_{1}} d y q_{\phi_{1}}^{\circ}\right] \\
& -\frac{8 \mathrm{~b}}{\mathrm{k}^{2} \ell} \sqrt{1-\mathrm{M}_{\mathrm{a}}^{2}}\left\{\int_{0}^{\ell} F_{\alpha}^{0} F_{\phi_{1}} \mathrm{E}_{\mathrm{v}_{1}} \mathrm{dy} \alpha+\int_{0}^{\ell} F_{\phi_{1}}^{0} F_{\phi_{1}} \mathrm{f}_{\mathrm{v}_{1}} \mathrm{dy} q_{\phi_{1}}^{0}\right\}
\end{aligned}
$$

All remaining $Q_{j+n, i}$ terms in (4-12b) will be zero.

For a given reduced frequency, computation of these generalized forces involves program output for both oscillating and steady flow conditions. Sectional generalized forces and pressure series coefficients output by the Rowe progran are used, in the same manner described above for steady chordwise loads, to compute (7-34). Integrals for oscillating flow, of course, are complex. Nine complex spanwise numerical integrations are needed for each $k$, whereas 126 would have been required without the simplifying approxinations. Two real integrations in $Q_{1+n, 1+2 n}$ involving steady pressures also appear in the steady displacement solution.

\section{B. Flutter Calculation Procedure and Results}

Incluston of subsonic three-dimensional (3-D) alrloads elfminates a serious flaw of the strip theory loads used to obtain all Chapter VI results. This was the approximate spanwise load distribution, which is 
most inaccurate near the tip. Leading-edge suction has been included In the same manner as the Chapter $V$, Section $D$, analysis of 2-D flow. An effect which was neglected in the strip theory case, that of the $x$-component of the resultant pressure force nomal to the deformed chord (cf. Eqs. (5-18) to (5-20) is now retained. It is accounted for by the chordwise terms which are computed with sectional generalized forces. The influence of Induced drag upon boti steady deformations and flutter stability should now be implicitly included by the modeling of chordwise loads. Two parameters which must be specified in addition to those mentioned in Chapter IV, in order to define a specific wing are the aspect ratio $\left(\frac{l}{b}\right)$ and Mach number.

The Chapter IV stablity calculation method has been modified to accept the subsonic 3-D steady and unsteady airloads derfved in Section A. Since these afrloads are now externally generated, 1teration of the reduced frequency to find neutrally damped eigenvalues is no longer feasible. Generalized forces have to be computed beforehand, both for steady flow and for oscillatory flow at preselected reduced frequencles. Computation of steady displacements for a given angle of attack and flight speed is accomplished as before, now based on Equations (7-31). Then for each of the preselected reduced frequencies, the previnusly calculated generalized forces (7-32) and (7-34) are input, the elgenvalue determinant (4-19) is assembled, and complex eigenvalues are determined. For each of these there are an associated speed and damping as shown by $(2-25)$ and $(2-27)$. The speed and damping of each flutter mode are then plotted for all $\mathrm{k}$, and a neutrally-damped speed $\mathrm{U}_{\mathrm{F}}$ is determined by graphlcal interpolation. The procedure is repeated 
with a newly estimated $U_{e}$; with care, $U_{e}$ and $U_{F}$ can be matched with sufficient accuracy after two such steps.

The expense in computer time to execute the Rowe program and the additional effort required to prepare the chordwlse loads make it desireable to use as few as possible $k$ values. Fortunately, all $U$ vs. I Interpolation graphs proved to be quite smooth. Flutter speeds accurate to three significant digits (sufficient) were reliably obtained.

The moderate-aspect-ratio wing whose stability boundary for 2-D airloads appears in Fig. 6-7 has been re-analyzed using 3-D incompressible aerodynamic theory. Figure 7-1 shows the results: compared with the 2-D flutter calculations. The curve marked "100\% suction" was computed with the complete system $(4-12),(7-32)$, and (7-34) whereas that inarked "0\% suction" was found by repeating the analysis after removal of all terms containing the singularity strength parameters $F_{\phi_{1}}$ etc. in $(7-34)$. This latter result thus represents the effects of forces normal to the deflected alrfoll chord only in the dynamic equations. The coupling of fore-and-aft bending motions into flutter by the leading edge suction forces is therefore absent in the "0\% suction" case.

The 2-D stability boundary Involves the aerodynamic loads of (4-18), which actually do not account for suction. The effect of mode1ing the suction force, as dertved in section $C$ of Chapter V, is shown by Figs. 6-35 and 6-36. These plots suggest that, If a stability boundary had been determined with suction accounted fors the 2-D curve In Fig. 7-1 would show less linfluence of steady deformation and would not drop below $U=7$. The solid, 3-D curve does, of course, account for suction. 
Comparison between the 2-D and 3-D flutter speeds is also made more difficult because they involve different steady aerodynamics. In order to minimize differences of this kind, the steady tip deflection was judged to be the best common measure of steady alrload effects. Inspection of Fig. 7-1 affirms that the influence of steady deformations upon flutter is not fust governed by elastic bending-torston coupling but is also sensitive to the manner in which the potential aerodynamic loads are applied to the fore-and-aft degree of freedom. Even though the chordwise force components represent tilting of a relatively large, approximately vertical resultant force vector, they are seen to have a significant stabilizing influence. This conclusion follows from comparing the "100\% suction" and " $0 \%$ suction" curves. Any analysis of this type will obviously be sensitive to the way in which chordwise forces are accounted for.

The influence of compressibility is next explored by repeating the foregoing calculations with airloads computed for Mach numbers of .6 and .8. Results are shown in Fig. 7-2. Strictly speaking, this procedure involves an inconsistency, since Mach number is held fixed while velocity is freely varied. The type of calculations required to model properly a wing at high subsonic speeds would require iterative matching of Mach number and speed $U_{F}$ for a given flight altitude. This refinement is deemed to be excessively costly in both computer time and effort. Nevertheless, the results of Fig. 7-2 provide interesting qualitative information on Mach number effects.

As $M_{a}$ is increased, the decrease in flutter speed with steady deformation for the "0\% suction" case becomes less pronounced. The 
results for " $100 \%$ suction" show less sensitivity to Mach number. In elther case, no adverse effects due to compressibility upon Elutter of a lifting wirg are revealed, other than those assoclated with a modest decrease in $U_{F}$ with increasing $M_{a}$.

When extending this analysis to higher subsonic Mach numbers, it must be remembered that the only aerodynamic loads being included are those which arise from inviscid, first-order, small perturbation steady and unsteady theory for planar lifting surfaces. Induced drag is present, as discussed above, but all. chordwise forces which arise from efther direct viscous shears or from modifications to the pressure distribution due to the presence of a boundary layer are not modeled. Yet viscous effects of this type become increasingly more pronounced as flight speeds apfroach the transonic regime and/or as mean angle of attack is increased.

The chordwise laods associated with dfrect viscous shear should not contain a significant unsteady component, at least at the lower reduced frequencies encountered here. It is expected that they will produce a steady drag force aligned with the airfoil section. By contrast, the chordwise potential loads represent the horizontal component of a relatively large resultant circulatory lift vector. Tilting of this vector can introduce unsteady chordwise loads of conslderable magnitude. Indeed their importance In aeroelastic stability is suggested by comparison of the $100 \%$ and $0 \%$ suction curves in the figures.

Since viscous shear should cause a predominantly steady drag loading, qualitative information regarding its effect on flutter can be inferred from the strip theory studies of Chapter VI involving the drag parameter 
C. For example, no substantial alteration of the dependence of flutter speed upon steady $11 \mathrm{ft}$ should be expected from including this additional drag term. The effect of unsteady viscous contributions to aeroeiastic stability has not been consldered anywhere in this investigation. The mere prediction of unsteady viscous chordwise loads is still regarded as an open question for experimental and analytical research, especially when turbulent boundary layers are involved.

As a final. case, the large-aspect-ratio example of Fig. 6-13 has been reanalyzed. Aspect ratio is fixed at 20 and Mach number at 0 . Results are shown in Fig. 7-3. Interestingly, the zero-1ift flutter speeds for 2-D and 3-D flows are nearly the same; of course, strip theory is expected to be more accurate for the larger aspect ratio. The difference between the 2-D and 3-D (with suction) stability boundaries is actually decelving, since (as already mentioned) the 2-D results do not contain the improved modeling of the suction force from Chapter V. Figures (6-37) and (6-38) suggest the stabilizing effect which Introducing suction would have upon the 2-D curve in Fig. 7-3. The key observation here is that the same type of instability, Involving substantial participation of the fore-and-att bending degree of freedom observed for 2-D aerodynamic loads, is still observed after 3-D aerodynamics are introduced. Also the influence of steady deformation upon flutter speed remains appreciable. Removal of the unsteady suction terms decreases stability, much as was observed for $\mathbb{R}=10$ in Fig. $7-1$. Points on the stability boundary for $\alpha<.005$ could not be found reliably because of the extremely light damplng in this region: approxlmate curves are shown by dashed lines. 
In conclusion, the results found in this chapter seem to confirm that the phenomena analyzed extensively In Chapter VI are still observed when the strip theory airloads are replaced by airloads from 3-D aerodynamic theory. Furthermore, the role of chordwise forces due to leading edge suction has been found to increase the aeroelastic stabilxty of a wing undargoing steady deformation due to lift. 


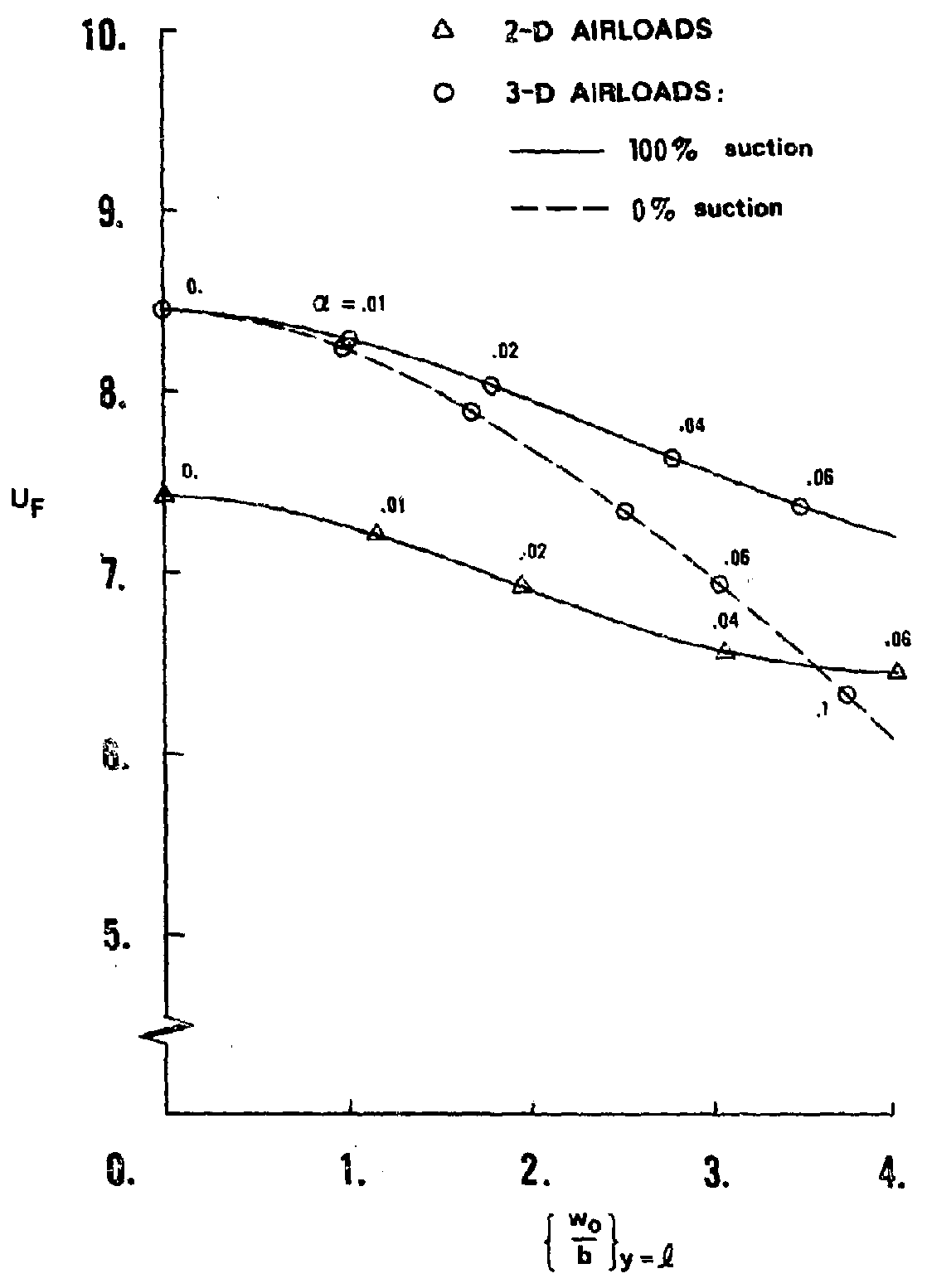

FIGURE 7-1 Flutter Speeds as Influenced by Steady Deformation, Moderate-Aspect-Ratio Example of Fig. 6-7 with $A=10$ and $M_{a}=0$ 


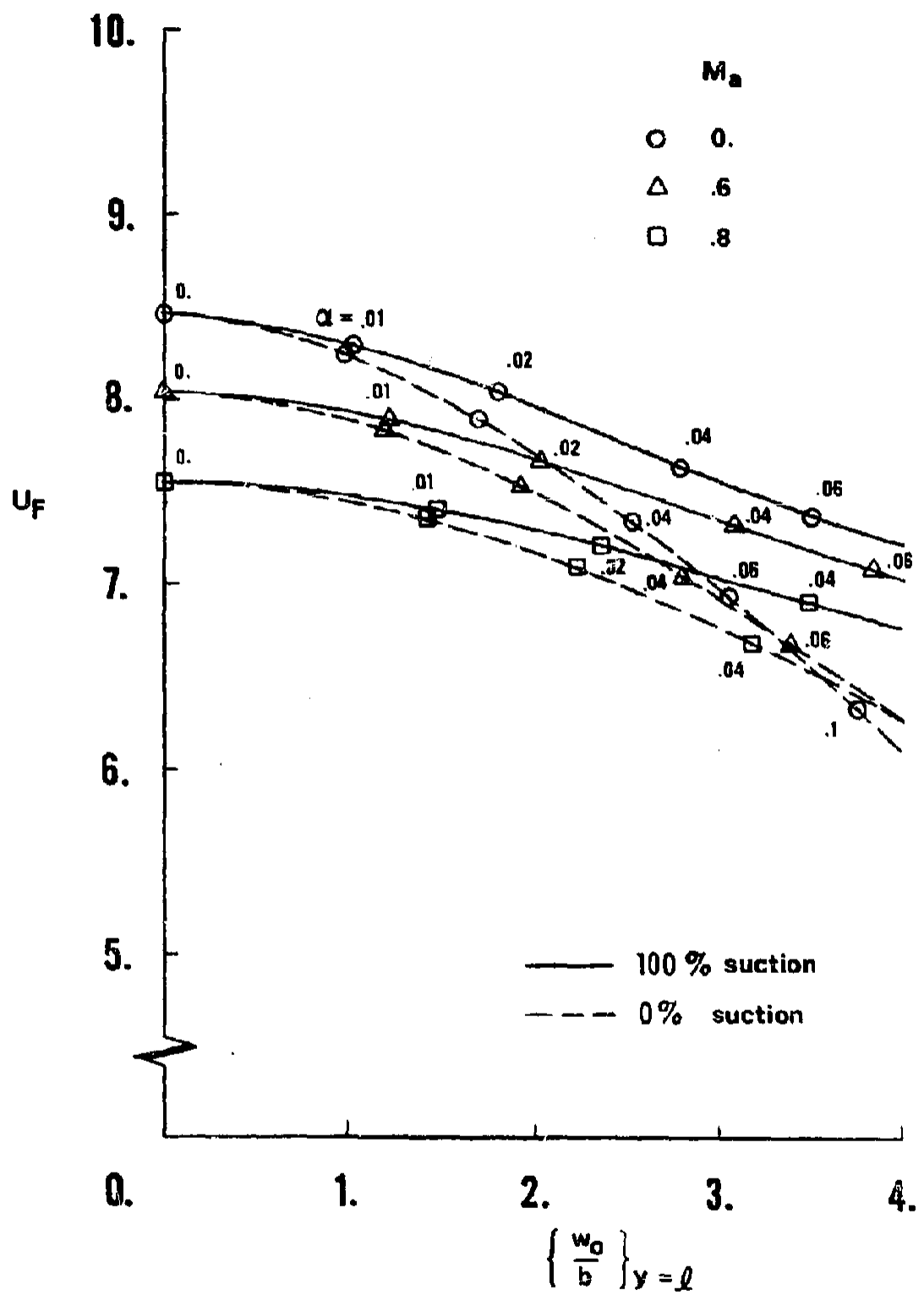

FIGURE 7-2 Flutter Speeds as Infiuenced by Steady Deformation at Three Mach Numbers; Wing of Fig. 7-1 


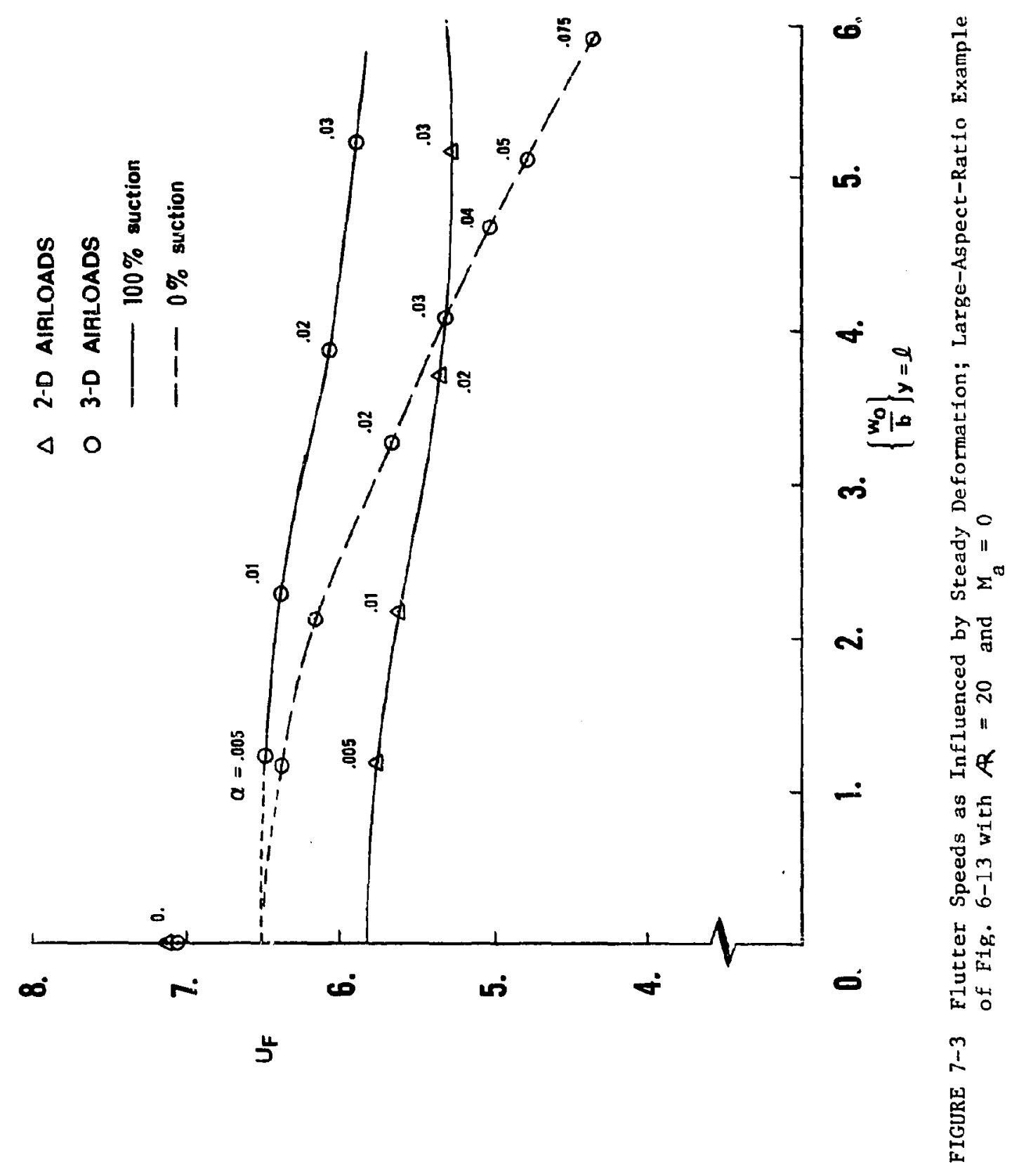




\section{Chapter VIII}

\section{CONCLUSIONS AND RECOMMENDATIONS}

The following conclusions are drawn from the varfety of results obtained in this investigation.

1) The influence of steady drag on flutter speed changes from favorable to unfavorable as aspect ratio is increased. The frequency of the second transverse bending mode decreases, tending toward the fundamental bending-torsion flutter frequency, as aspect ratio is increased.

2) The prediction of the influence of steady drag upon flutter is not substantially altered when steady defcrinations due to lift axe considered. The major effect of steady drag is to reduce divergence speed, especially for large aspect ratios.

3) When a wing has such a large aspect ratio that its fundamental fore-and-aft bending frequency is less than the frequency of bendingtorsion flutter at zero steady lift, an instability associated with chordwise bending occurs. The critical. speeds are lower than the zero-lift flutter speed when any steady lifting deformation is present. This type of flutter can disappear at small steady deflections when realistic structural damping is introduceo, but for reasonably large displacements it can still occur.

4) Steady deformations decrease flutter speed and flutter frequency. The effect is most pronounced when the fundamental fore-and-aft bending frequency is near the zero-lift bending-torsion flutter frequency.

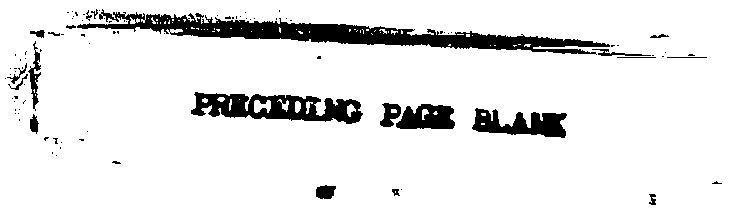


5) The aeroelastl: phenomena predlcted using incoimpressible striptheory airloais are also abserved when three-dimensional, compressible subsonic astrloads are employed.

6) The inciusion of unsteady leading-edge euction forces moderates the predicted dectease in flutcer speed due to steady lifting detormations.

7) In subsonfc compressible flow, with unsteady potentlal chordrise forces, there seem to be only slight adverse effects of $M_{a}$ on Flutter when steady deformations are present.

One of the predictions of chis study is that a high performance sailplane undergoing a litut load factor pullout from a dive could encounter fluter or divergence at lower speeds than might be anticipated from a conventional aeroelastic analysis. The increased sceady drag which accompanies higher $c_{L}$ would reduce the divergence speed considerably, while the deformation of the flextble high-aspect-ratio wings would change the dynamic aeroelastic atablity as well.

The present analys is could be refined still further. Nonlinear aerodynamic effects deserve further atcention. Higher mean angles of attack would leat to increased importance of the turbulent boundary layer, culminating in separation (stall), which wl11 alcer tie stabilizing contribution found due to leading-edge suction in attached flas. Vehicles intended to eperate at the higher subsonic or low transanic speeds (certain RPF' or missiles could still be designed wich the straight wings constdered here) are expected to encounter various phenomena which could greatly affect the chorduse loads. Viscous 
shear, boundary Jayer nodification of the flow, and thickness effccts with the appearance of shocks will all modify the aeroelastic behavior. Finaliy, the transonic and supersonic flow regimes, where drag loads are considerably larger than at subcritical speeds, remain largely uninvestIgated. In these ranges different structural and aerodynamic configurations are likely to be involved. 
Appendix A

THE ASSUMED MODES

\section{Bending}

The natural mode shapes and frequencies In bending of a uniform beam of length $\ell$ are found by seeking homogeneous simple harmonic solutions of

$$
E I_{X} w^{n ! \prime}+m \ddot{w}=0
$$

Letting

$$
w=w e^{I \omega t}
$$

the general solution is

$$
w=A_{1} \sin \sqrt{\frac{\omega}{a}} y+A_{2} \cos \sqrt{\frac{\omega}{a}} y+A_{3} \sinh \sqrt{\frac{\omega}{a}} y+A_{4} \cosh \sqrt{\frac{\omega}{a}} y
$$

where $\quad a^{2}=\frac{E I x}{m}$

Application of the bending boundary conditions

$$
W(0)=W^{\prime}(0)=W^{\prime \prime}(l)=W^{\prime \prime \prime}(l)=0
$$

results in a trancendental equation for the natural frequency eigenvalues

$$
\cos \sqrt{\frac{\omega}{2}} \ell=-\frac{1}{\cosh \frac{\omega}{a} \ell}
$$

which are for vertical bending

$$
\text { (A-1) } \quad \omega_{w_{i}}=\Pi^{2} N_{1}^{2} \frac{1}{\ell^{2}} \sqrt{\frac{E I}{m}} \quad i=1,2,3, \ldots
$$

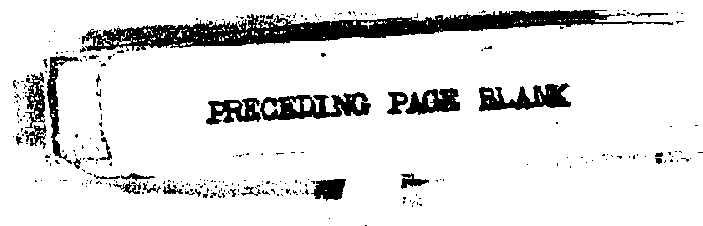


The trancendental numbers $\mathrm{N}_{1}$ for $1 \leq \mathrm{i} \leq 5$ are

$$
\begin{aligned}
\mathrm{N}_{1} & =0.596864162695 \\
\mathrm{~N}_{2} & =1.494175614274 \\
\mathrm{~N}_{3} & =2.500246946168 \\
\mathrm{~N}_{4} & =3.499989319849 \\
\mathrm{~N}_{5} & =4.500000461516
\end{aligned}
$$

and the corresponding eignevectors yield the vertical bending natural modes

$(A-3) \quad E_{w_{i}}=\left[\left(\frac{\sin N_{i} \Pi-\sinh N_{i} \Pi}{\cosh \Pi N_{i}+\cos \Pi N_{i}}\right)\left(\sinh \pi N_{i} \tilde{y}-\sin \Pi N_{i} \tilde{y}\right)\right.$

$\left.+\cosh \Pi N_{i} \tilde{y}-\cos \pi N_{i} \tilde{y}\right]$

expressed in orthonormal form so that

$$
\int_{0}^{1} f_{w_{j}}^{2}(\tilde{y}) d \tilde{y}=1
$$

The modal property $B_{i}$ in the steady equations $(4-9)$ is related to the modal integral

$$
\int_{0}^{1} f_{w_{i}} d y=-\frac{i}{N_{i} \pi}\left[\frac{\sin \pi N_{i}-\sinh \pi N_{i}}{\cosh } \frac{\pi N_{1}+\cos \pi N_{i}}{\pi} \equiv-\frac{B_{i}}{2}\right.
$$

Fore-and-aft bending nacural modes $f_{v_{i}}$ are the same as in $(A-3)$, however the natural frequencies are

$(A-5)$

$$
\omega_{v_{i}}=\Pi^{2} N_{i}^{2} \frac{1}{l^{2}} \sqrt{\frac{E 2}{m}}=\sqrt{\tau} \omega_{w_{1}}
$$


The $f_{w_{1}}$ are 111 ustrated in FIg. A-I for $1 \leq 1 \leq 5$.

\section{Torsion}

Natural mode shapes and frequencies in torsion are simply

$(A-6)$

$$
\hat{x}_{\phi_{j}}=\sin \Pi\left(j-\frac{1}{2}\right) \tilde{y}
$$

$(A-7)$

$$
\omega_{\phi_{j}}=\Pi(j-i \xi) \frac{1}{l} / \frac{\sqrt{\mathrm{GI}_{d}}}{\mathrm{~J}}
$$

which result from the elementary Sturm-Liouville problem for torsion of a unirorm rod. These modies are not normalized since

$$
\int_{0}^{1} f_{\dot{\Phi}_{j}}^{2}(\tilde{y}) d \tilde{y}=1 \underline{\underline{z}}
$$




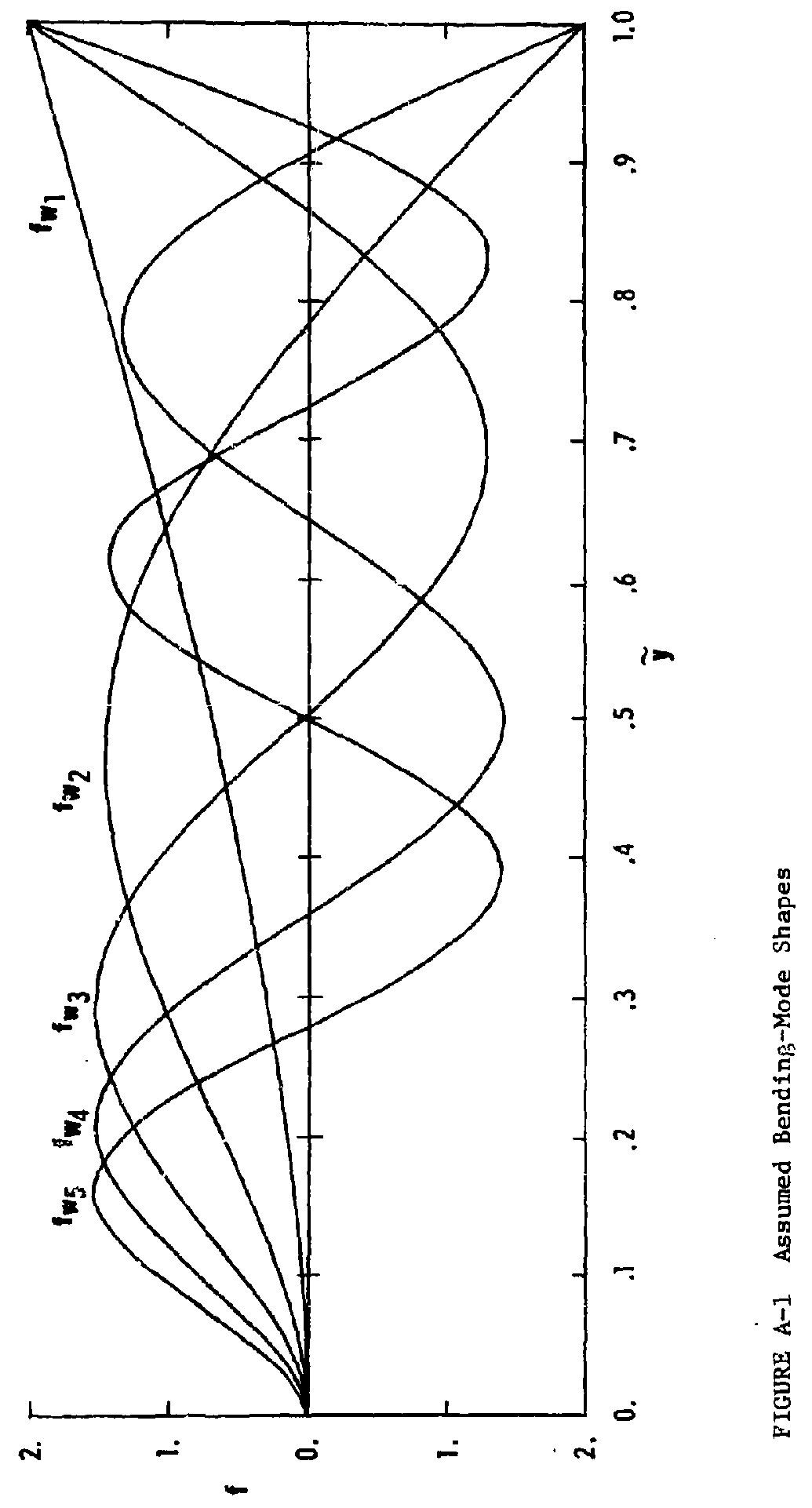


CALCULATION OF MODIFIED BESSEL FUNCTIONS

The modified Bessel functions $K_{0}(\bar{s})$ and $K_{1}(\bar{s})$ appearing in the expression (5-1) for the generalized Theodorsen function can be computed by using the following ascending power sertes expansions, drawn from Abramowitz and Stegun (Ref. 26, Equations 9.6.10, 9.6.11, and 9.6 .13$)$.

$(\mathrm{B}-1)$

$$
I_{v}(\bar{s})=\left(\frac{1}{2} \bar{s}\right)^{\nu} \sum_{j=0}^{\infty} \frac{\left(\frac{\left.14 s^{2}\right)^{j}}{j ! \Gamma(v+j+1)}\right.}{j !(v)}
$$

$(\mathrm{B}-2)$

$$
\begin{aligned}
\mathrm{K}_{0}(\overline{\mathrm{s}})= & -\left\{\ln \left(\frac{1}{\mathrm{~s}} \overline{\mathrm{s}}\right)+\gamma_{\mathrm{e}}\right\} \mathrm{I}_{\mathrm{o}}(\overline{\mathrm{s}}) \\
& +\frac{\frac{1}{4} \bar{s}^{2}}{(1 !)^{2}}+\left(1+\frac{1}{2}\right) \frac{\left(\frac{1}{4} \mathrm{z}^{2}\right)^{2}}{(2 !)^{2}}+\left(1+\frac{1}{2}+\frac{1}{3}\right) \frac{\left(\frac{1}{4} \mathrm{z}^{2}\right)^{3}}{(3 !)^{2}}+\ldots
\end{aligned}
$$

$$
\begin{aligned}
k_{1}(\bar{s})= & \frac{1}{2}\left(\frac{1}{2} \bar{s}\right)^{-1}+\ln \left(\frac{1}{2} \bar{s}\right) I_{1}(\bar{s}) \\
& -\frac{1}{2}\left(\frac{1}{2} \bar{s}\right) \sum_{j=0}^{\infty}\{\psi(j+1)+\psi(j+2)\} \frac{\left(1 / \bar{s}^{2}\right)^{j}}{j !(j+1) !}
\end{aligned}
$$

where

$$
\begin{aligned}
\Gamma(\nu+1) & =\nu ! \\
\psi(1) & =-\gamma_{\mathrm{e}} \quad \nu-1 \\
\psi(\nu) & =-\gamma_{\mathrm{e}}+\sum_{j=1} j^{-1}, v \geq 2 \\
\gamma_{\mathrm{e}} & =0.5772156649 \ldots \quad \text { (euler's constant) }
\end{aligned}
$$

No convergence difficulties with the power series expansions were encountered over the range of $\bar{s}$ that occurred in this investigation. 
Simple harmonic airloads involving the Theordorsen function of reduced

frequency $C(k)$ were computed by the same procedure, simply by the substitution $\bar{s}=0+1 k$. 
SOLUTION FOR NONLINEAR STEADY DISPLACEMENTS

With the vector of generalized displacements defined as

$(\mathrm{C}-1)$

$$
\dot{q}^{\circ}=\left\{q_{w_{1}}^{0}, \ldots, q_{w_{n}}^{0}, q_{v_{1}^{0}}^{0}, q_{v_{n}}^{0}, q_{\phi_{1}}^{0}, \ldots, q_{\phi_{n}}^{0}\right\}^{T}
$$

the nonlinear equations (4-9) can be expressed,

$(C-2) \quad F\left(q^{\circ}\right)=0$

where

(C-3) $\quad F=\left\{F_{w_{1}}, \ldots F_{w_{n}}, F_{v_{1}}, \ldots, F_{v_{n}}, F_{\phi_{1}}, \ldots, F_{\phi_{n}}\right\}^{T}$

Let an initial estimate $q_{\sim}^{\circ}(0)$ be found by solving the linear steady equations (4-20), then linearize $\underset{\sim}{\mathbf{F}}$ about $\underset{m}{\mathbf{q}^{\circ}}(0)$ by first-order Taylor serles,

$(\mathrm{C}-4) \quad \underset{\sim}{\mathrm{F}}\left(\mathrm{q}^{0}\right)=\mathrm{F}\left(\mathrm{q}^{0}(0)\right)+\left[J\left(\mathrm{q}_{(0)}^{0}\right)\right]\left(\mathrm{q}^{0}-\underline{\sim}_{(0)}^{0}\right)+\mathrm{H} .0 . \mathrm{T}$.

The Jacobian matrix. $J$ contains partial derivatives of the $F^{\prime} s$ with respect to the $q^{\circ} s$ evaluated at $q^{\circ}(0) ;$ its elenents are shown in Fig. C-1. Equation (C-4) can be used to solve for $\left(q_{\sim}^{0}(1)-\underset{\sim}{q}(0)\right)$ by the Iinear approximation

(c-5) $\quad F\left(q_{(0)}^{\circ}\right)+\left[J\left(q_{i}^{\circ}\right)\right]\left(q_{(1)}^{\circ}-q_{m}^{\circ}(0)\right)=0$

and the first iteration solution is

(c-6) $\quad \underline{q}_{(1)}^{\circ}=\left(q_{(1)}^{\circ}-\underline{q}^{\circ}(0)\right)+q^{\circ}(0)$ 
This process is repeated untll gatlafactory convergence is achieved, with the general jth step Biven by

$$
\begin{aligned}
& \text { (C-7) }\left[J\left(q_{(j)}^{0}\right)\right]\left(q_{m}^{0}(j+1)-q_{m}^{0}(j)\right)=-F\left(q_{(j)}^{0}\right) \\
& q_{m}^{0}(j+1)=\left(q^{0}(j+1)-q^{0}(j)\right)+q^{0}(j)
\end{aligned}
$$

In practice, nore than four or five iterations rarely were required, for cases near static divergence speeds. 


$$
\begin{aligned}
& J_{j, 1}=\frac{\partial F_{w_{1}}}{\partial q_{w_{i}}^{\circ}}=\Pi^{4} N_{j}^{4} \frac{M P I_{\alpha}}{b U^{2}} \delta_{1 j}+(\tau-1) \frac{M P I_{\alpha}}{b^{2}} \sum_{\mu=1}^{n} \sum_{v=1}^{n} R_{1 j \mu v} q_{\phi_{\mu}}^{\circ} q_{\phi_{v}}^{\circ} \\
& J_{j, i+n}=\frac{\partial F_{w_{j}}}{\partial q_{v_{1}}^{0}}=-\frac{M P I_{\alpha}}{b U^{2}}(\tau-1) \sum_{v=1}^{n} H_{v j I} q_{p_{v}^{0}}^{0}
\end{aligned}
$$

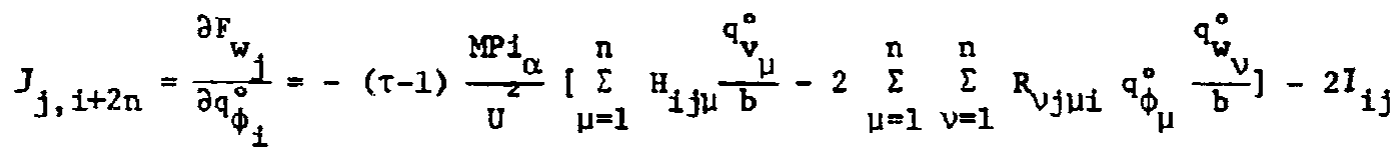

$$
\begin{aligned}
& J_{j+n, i}=\frac{\partial F_{j}}{\partial q_{w_{1}}^{\circ}}=-\frac{M^{\prime} I_{\alpha}}{b U^{2}}(\tau-1) \sum_{v=1}^{n} H_{v i j} q_{\Phi_{v}}^{\circ}
\end{aligned}
$$

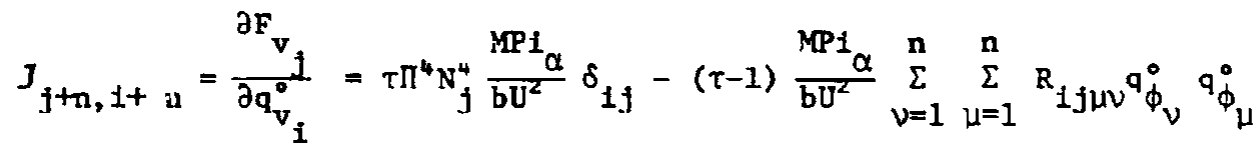

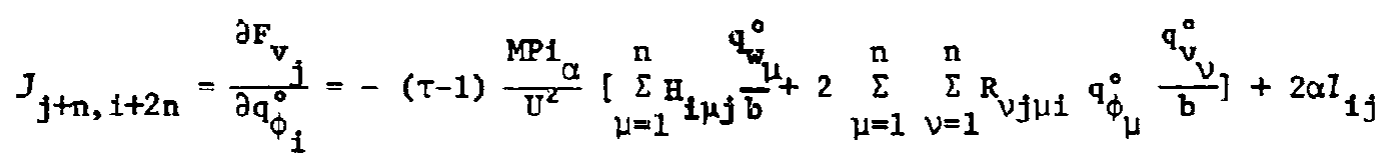

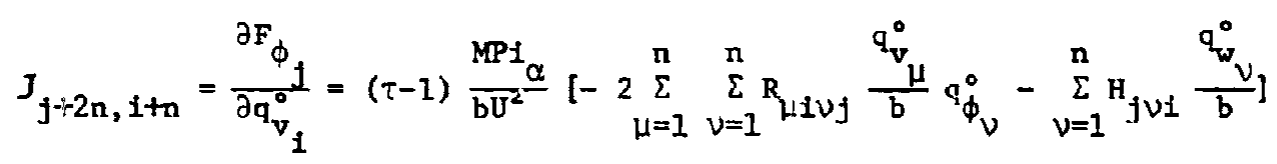

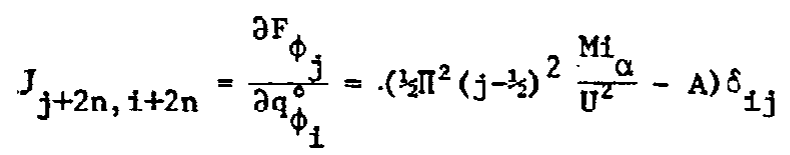

$$
\begin{aligned}
& +(\tau-1) \frac{M P i_{\alpha}}{U^{2}}\left[\sum_{\nu=1}^{n} \sum_{\mu=1}^{n} R_{\mu \nu j i} \frac{q_{w}^{\circ}}{b} \frac{q_{w}^{\circ}}{b}-\sum_{\nu=1}^{n} \sum_{\mu=1}^{n} R_{\mu \nu j 1} \frac{q_{v}^{\circ}}{b} \frac{q_{v}^{o}}{b}\right] \\
& \delta_{i j}= \begin{cases}1 & (i=j) \\
0 & (i \neq j)\end{cases} \\
& (1 \leq 1 \leq n),(1 \leq j \leq n)
\end{aligned}
$$

FIGURE C-1 Elements of the Jacobian Matrix 
REFERENCES

1. Petre, A. and H. Ashley, "Drag Effects on thing Flutter," J. of Alrcraft, Vol. 13, No. 10, October 1976, pp. 755-763.

2. Petre, A., Teoria Aeroelastcitat11-Statica, Editura Academiel Republicil Socialiste Romania. Bucharest, 1966.

3. Petre, A., "L'effet de la résistance à $l$ 'avancement sur la vitesse critique du flutter," Proceedings Sixth International Congress of the Aeronautical Sciences. Munich, September 1968.

4. Hoff, N. . The Analys is of Structures, John W1ley and Sons, New York, 1956 .

5. Bisplinghoff, R.L. and Ashley, H. Principles of Arroelasticlty. John Wiley and Sons, New York, 1962.

6. Houbolt, J.C. and Brooks, G.W., "Differential Equations of Motion for Combined Flapwise Bending, Chordwise Bending, and Torsion of Twisted, Nonuniform Rotor Blades," NACA Report No. 1346. 1958.

7. Hodges, D.H., and Dowell, E.H., "Nonlinent Equations of Motion for the Elastic Bending and Torsion of Tristed Nonuntform Rotor Blades." NASA TN D-7818, 1974.

8. Irwin, C.A.K. and P.R. Guyett, "The Subcrtstcal Response and Flutter of a Swept King Madei,:" Tech. Kept. 65186, RAE, Farnborough, U.K., August 1965.

9. Goland, M. and Luke, Y.I., "A Study of the Bending-Torsion Aeroelastic Modes for Aircraft Wings," J. Aero. Sc1.. Vol. 16, אo. 7. July 1949, pp. 389-396.

10. Jones, R.T., "Operational Treatment of the Non-Uniform deift Theor: to Airplane Dynamics," NACA TN 667, 1938.

11. Edwards, J.H., "Unsteady Aerodynamic Modeling and Active Aeroelastic Control," SUDAAR S04, February 1977.

12. von Kármán, T. and Burgers, J.M.. General Aerodynamic Theory -Perfect Fluids, Vol. II of Aerodynamic Theory, div. E. W.F. Durand. ed: ; Inlius Springer (Berlin), 1935.

13. Greenberg. J.M., "Some Considerations on an Aftoil in an Oscillaring Stream," NACA TN 1372, August 1947.

14. Garrick, I.E., "Propulsion of a Fiapping and Oscillating Alrfoll." NACA Report No. 567, May 1936.

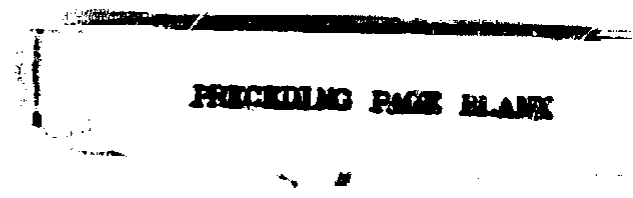


15. Welch, A., Welch, L., and Irving, F., New Soarlng Pilot. Butler and Tanner L.td., London, 1968.

16. Marsden, D.J., "Gemini-- A Variable Geometry Sailplane," AIAA, MIT. and SSA 2nd International Symposium on the Technology and Science of Low Speed and Motorless Flight, Cambridge, Mass., September $11-13,1974$.

17. Coetz, R.C.. "Divergence of Some All-Movable Coritrol Surfaces Including Drag Loadings." NASA TN D-4793, Octéoer 1968.

18. Rowe, H.S., Redman, M.D., Ehters, F.E., and Sebastian, J.D., "Prediction of Unsteady Aerodynamic Londjngs Caused by Leading Edge ard Trailing Fdge Conerol Surface tot lons in Subsonte Compressible F:ow-- Analysis and Results," MSA CR-2543, 1975.

19. Redman, M.C. and Rowe. W.S. "PredJcelon of Unsceady Merodynamic Loadings Caused by Leading Edge ard Tratling Edge Concrol Surface Motions in Subsonic Compressible Flow- Computer Prograo Description." SASA CR-132636, Hay 1975.

20. Novozhilov, V.V.. Foundations of the Ronllnear Theory of flastlclev. Translated edition by rraylock Press, Rochester. New York, 1953.

21. Sears, H.R., "Operat tonal Kethods in the Theory of Airfolls in Non-Inifor Yotiun", J. of the Franklin Inst., Vol. 230, No. 1. July 1940, pp. 95-11.1.

22. Mllne. R.D. "Asympot ic Solut lons of Linear Stat lonary InterroDffferential Equations," A.R.C.R. \& Y. 3568, July 1966.

23. Bisplinghof?. R.L., Ashley. H., and Hal faon. R.L., teroelasticley. Add Ison-Mesley. Reiding. Mnss. 1955.

24. Hatkins, C.E., Ruryan, H.L.. and Woolston, D.S., "On the kernei Funct Ion of the Integral Equat lon Relating the LIft and Dowmash Diseributions of Osclllating Finfte Ningt in Subsonic Flow," xach Rep. 1234.

25. Jones, R.T. and Cohen, D., High Speed ying Theory. Princecon deronautical Paperback tio. 6. Princeton tiniversity Press. Priniczon, 1960.

26. Handbook of Mathemetcal Functlons (ed. by Abramouttz and Stegun), Dover, Sew Fork, 1965.

27. Johnson. Wayne. "Dynatics of Tilt 2 ne Proprotor Alreraft in Crulse Flight," sish TN D-7677. May 1974. 


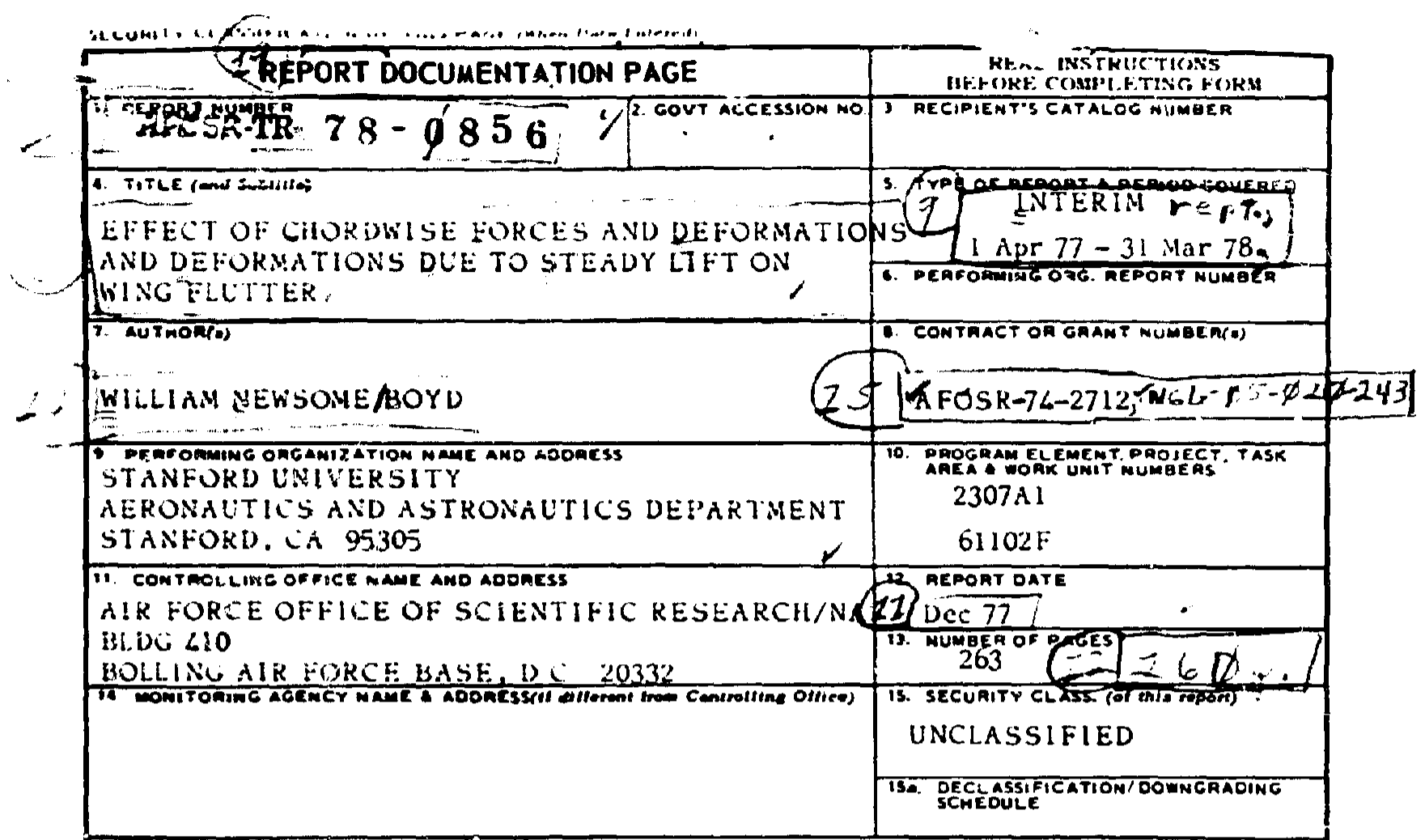

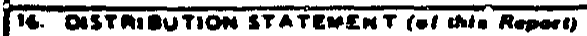

ipproved for pubilic release: distriburion unlimited.

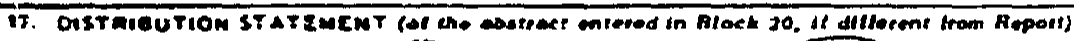

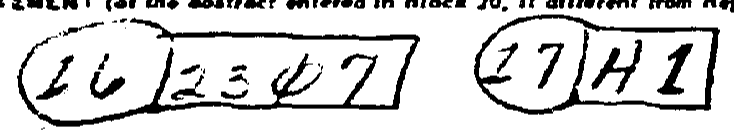

16. SUPPLEMENTAMY NOTES

$$
-7[i+i n+1-578
$$

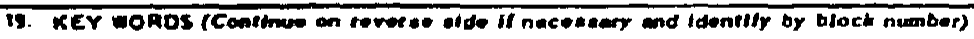

AEROELASTICITY

STATIC STABHITY

DYNAMIC STABIITY

CHORIWISH FOREE

IARCiE ASPECT RATIO Hi NGiS

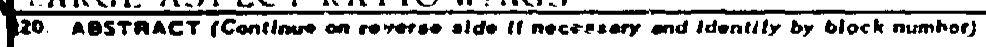

This investigation explores the effects of chordwise forees and deformations and steadyslate deformation due to lift on the static and dynamic acroclastic stabitity of a uniform cantilever wing. Results of this analysis are believed to have pracical applications for high-performance sailplanes and certain RPV's. The airfoil cross section is assumed to be symmetric and camber bending is neglected. Motions in vertical bending, fore-andaft bending, and torsion are considered. A differential equation model is developed, which included the nonlinear elastic bending-torsion coupling that accompanies even moderate deflections, A linearized expansion in small time-dependent deflecions is madef,

DD, FonM 1473 EDITION of i nov 65 is obsolete


wiout a steady flight cendition. The stability delerminant of the linearized system then cuntains coefficients that depend on steady displacements. Loads derived from twodimensional incompressible aerodynamic theory are used lo obtain the majority of the resulis, but cases using three-dimensional subsonic compressible theory are also studied The stability analysis is carried out in terms of the dynamically uncoupled natura! modes of vibration of the uniform cantilever Dynamic stability in the case of incompressible strip-theory airloads is determined in two ways. One is the " $\mathrm{V}-\mathrm{g}$ method" familiar to aeroelasticians. When steady deformations are present this methnd requires an iterative matching of flutter speeds with estimated speed. The second approach involves determination of the complex eigenvalues of the aerpelastic modes at any desired flight condition. The aerodynamic loads are expressed in terms of the generalized Theodorsen function: eigenvalues of the aeroclastic system are localed with a gradient search lechnique.

The eifeet of steady drag on flutter of nonlifting wings using incompressible strip-theory is studied and shown to correlate with previousl known results. Next, the influence of steady lifting deformations on flutter is investigated, and flutter modes are found that involve fore-and-aft bending motions. The significance of unsteady leading edge suction iorces, which are predicted by the two-dimensional incompressible aerodynamic theory. is then examined. Two idealized examples based upon existing sailplanes are analyzed. Steady drag loads lower the flutter speed for larger aspect ratios but increase it for aspect rotios less than a certain value. Divergence speed is more sensitive to steady drag. and for very high aspect rotio wings it can fall below the bending-torsion flutter speed. Steady deformations due to lift always decrease the flutter speed by an amount dependent upon the aspect ratio and the fore-and aft bending stiffness. Leading-edge suction torces inc-ease flutter speed. Three-dimensional steady and unsteady airlcads are introduced into the $V-g$ flutter analysis scheme, and for a Mach number of zero the role of steady lifting defcrmations and unsteady leading-edge suction forces is more accurately determined. The behavior predicted using strip theory loads is again observed. and the suction forces are confirmed to contribute a significant slabilizing effect. Further calculations using high subsonic Mach numbers reveal only mild effects due to compressibility (disregarding unsteady cordwise loads of viscous origin). 